University of Tennessee Health Science Center

UTHSC Digital Commons

\title{
$5-2013$
}

\section{Gene Therapy and Stem Cell Therapy to Improve the Outcome of Human Islet Transplantation}

$\mathrm{Hao} \mathrm{Wu}$

University of Tennessee Health Science Center

Follow this and additional works at: https://dc.uthsc.edu/dissertations

Part of the Medical Genetics Commons

\section{Recommended Citation}

Wu, Hao , "Gene Therapy and Stem Cell Therapy to Improve the Outcome of Human Islet Transplantation" (2013). Theses and Dissertations (ETD). Paper 300. http://dx.doi.org/10.21007/etd.cghs.2013.0358.

This Dissertation is brought to you for free and open access by the College of Graduate Health Sciences at UTHSC Digital Commons. It has been accepted for inclusion in Theses and Dissertations (ETD) by an authorized administrator of UTHSC Digital Commons. For more information, please contact jwelch30@uthsc.edu. 


\title{
Gene Therapy and Stem Cell Therapy to Improve the Outcome of Human Islet Transplantation
}

\begin{abstract}
Since its first introduction in 1990s, the Edmonton Protocol for human islet transplantation has helped more than 500 patients with type 1 diabetes worldwide and met great success from bench to bedside. The Edmonton Protocol involves isolating islets from a cadaveric donor pancreas by enzymatic digestion and infusing into the patient's portal vein. Each recipient usually receives islets from two or three donors for an optimal outcome and is dosed with two immunosuppressive drugs, sirolimus and tacrolimus and a monoclonal antibody drug daclizumab to prevent the graft rejection. However, despite the administration of immunosuppressive drugs and the recent improvement in islet isolation, preparation and transplantation, insulin independence is rarely sustained for long term after islet transplantation. It was reported that by a year after transplant, $50-68 \%$ of patients do not need to receive additional insulin, but by five years after the procedure, few than $10 \%$ of total patients are free of daily insulin injection.

The wide application of human islet transplantation is hindered by two major barriers, the immune rejection from the organ recipients and the PNF of islet grafts. Immune rejection describes a process where transplanted islets are attached, recognized and attacked by the host immune system while the PNF is characterized as the loss of islet viability and function caused by non-immune reasons, such as the disruption of islet microvasculature during islet isolation and purification process, hypoxia in the core of islet grafts and production of inflammatory cytokines at the transplantation sites.
\end{abstract}

Several strategies have been proposed to address the immune rejection and the PNF. Gene therapy, which relies on "vectors" to deliver therapeutic genes into human islets, was successful in immunodeficient animal models because the potent expressions of therapeutic proteins usually lead to an advantage in the post-transplantation survival of human islets. However, gene therapy provided limited success to overcome the immune rejection. Also gene therapy faced inherited problems such as the low transfection efficiency of non-viral vectors and the increasing safety concerns of the viral vectors.

Cell therapy especially stem cell therapy, on the other hand, has recently met great success as a novel regenerative medicine to support solid organ transplantation including human islet transplantation. Among all types of stem cells, mesenchymal stem cells (MSCs) are given special interest for their selfrenewal potential, multi-lineage capacities, paracrine effects (trophic mediator) and immune modulatory effects, making it a great candidate for improving human islet transplantation. MSCs are mostly found in bone marrow, adipose and umbilical cord blood, are one of the most extensively studies adult stem cells used in treating degenerative diseases as well as solid organ transplantation. Unlike embryonic stem cells (ESCs) or induced pluripotent stem cells (iPSCs), adult stem cells show restricted proliferation and lineage differentiation, and consequently have less risk of inducing tumor. MSC-based therapy has been used to improve human islet transplantation from three major aspects. First, MSC was used as an immune modulator to prevent the immune rejection of human islets. Second, MSC was used as a trophic mediator to improve the revascularization of human islets. Third, MSC was induced in vitro to form insulin producing cell as an alternative sources of human islets. The third aspect seems to be quite promising theoretically, but no group has successfully generated enough "artificial" islets for human transplantation. Our focus is mainly on the immunomodulatory effects and the trophic effects of MSCs to improve the human islet transplantation.

We also introduce humanized mouse model into the studies of islet transplantation. Humanized mouse model contains mature human immunity and is a good model to determine the immune rejection to human islet after transplantation. Our study is innovative because this is the first time humanized animal is used test the efficacy of MSC based regenerative medicine on solid organ transplantation. We plan to 
answer the following questions in this study. 1) Can humanized mouse model pose an immune rejection to human islets after transplantation? 2) What is the duration and limitation of the adoptively transferred human immunity? 3) What is the efficacy and mechanism of stem cell therapy in improving human islet transplantation in humanized mouse model?

\section{Document Type}

Dissertation

Degree Name

Doctor of Philosophy (PhD)

\section{Program}

Pharmaceutical Sciences

\section{Research Advisor}

Ram I. Mahato, Ph.D.

\section{Keywords}

Gene Therapy, Human Islet Transplantation, Immune Rejection, Stem Cell Therapy

\section{Subject Categories}

Medical Genetics | Medical Sciences | Medicine and Health Sciences 


\title{
Gene Therapy and Stem Cell Therapy to Improve the Outcome of Human Islet
} Transplantation

\author{
A Dissertation \\ Presented for \\ The Graduate Studies Council \\ The University of Tennessee \\ Health Science Center \\ In Partial Fulfillment \\ Of the Requirements for the Degree \\ Doctor of Philosophy \\ From The University of Tennessee
}

By

$\mathrm{Hao} \mathrm{Wu}$

May 2013 
Portions of Chapter 1 (C) 2011 by American Chemical Society. Portions of Chapter 1 (C) 2013 by Springer.

Portions of Chapter 3 (c) 2010 by American Chemical Society. Portions of Chapter 4 (c) 2011 by John Wiley \& Sons.

Portions of Chapter 5 (C) 2011 by Springer.

Portions of Chapter 6 @ 2013 by Nature Publishing Group. All other material (C) 2013 by Hao Wu.

All rights reserved. 


\section{ACKNOWLEDGEMENTS}

My thanks and appreciation to Dr. Ram I Mahato for tutoring and supporting me as my academic supervisor throughout the time it took me to complete this research and write the dissertation. The inspiration for doing the research came from his insightful minds and five years PhD study was one of the most important experiences in my life. I am grateful as well to Brenda Thornton, Betty Bowman, Harriet Lang and Cynthia Crowe for coordinating and overseeing the administrative concerns that made it possible for me to complete my degree.

The members of my dissertation committee, Dr. Duane Miller, Dr. Bernd Meibohm, Dr. Yi Lu and Dr. Ivan Gerling have generously given their time and expertise to better my work. I thank them for their contribution and support.

I am grateful to many persons who shared their expertise and experiences. Dr. Scott Jackson, Dr. David Hamilton from Department of Comparative Medicine and Dr. Luis Campos from Methodist University Hospital generously shared their meticulous expertise in animal surgery which greatly supported and expanded my research. Dr. Meifeng Lu and Dr. Dan Rosson kindly provided technical support to my research. Dr. David Armbruster has revised all my manuscripts and gave me the courage and the skills to write and present as a non-native English speaker.

I must acknowledge as well the many friends, colleagues, students, teachers, and other librarians who assisted, advised, and supported my research and writing efforts over the years. Especially, I need to express my gratitude and deep appreciation to my wife Chengcheng Liu whose understanding, wisdom, and sense of humor have supported, enlightened, and entertained me over many years. She has consistently helped me keep perspective on what is important in life and shown me how to deal with reality.

I thank the National Institutes of Health (NIH) for financial support (RO1 DK69968). 


\begin{abstract}
Since its first introduction in 1990s, the Edmonton Protocol for human islet transplantation has helped more than 500 patients with type 1 diabetes worldwide and met great success from bench to bedside. The Edmonton Protocol involves isolating islets from a cadaveric donor pancreas by enzymatic digestion and infusing into the patient's portal vein. Each recipient usually receives islets from two or three donors for an optimal outcome and is dosed with two immunosuppressive drugs, sirolimus and tacrolimus and a monoclonal antibody drug daclizumab to prevent the graft rejection. However, despite the administration of immunosuppressive drugs and the recent improvement in islet isolation, preparation and transplantation, insulin independence is rarely sustained for long term after islet transplantation. It was reported that by a year after transplant, $50-68 \%$ of patients do not need to receive additional insulin, but by five years after the procedure, few than $10 \%$ of total patients are free of daily insulin injection.

The wide application of human islet transplantation is hindered by two major barriers, the immune rejection from the organ recipients and the PNF of islet grafts. Immune rejection describes a process where transplanted islets are attached, recognized and attacked by the host immune system while the PNF is characterized as the loss of islet viability and function caused by non-immune reasons, such as the disruption of islet microvasculature during islet isolation and purification process, hypoxia in the core of islet grafts and production of inflammatory cytokines at the transplantation sites.
\end{abstract}

Several strategies have been proposed to address the immune rejection and the PNF. Gene therapy, which relies on "vectors" to deliver therapeutic genes into human islets, was successful in immunodeficient animal models because the potent expressions of therapeutic proteins usually lead to an advantage in the post-transplantation survival of human islets. However, gene therapy provided limited success to overcome the immune rejection. Also gene therapy faced inherited problems such as the low transfection efficiency of non-viral vectors and the increasing safety concerns of the viral vectors.

Cell therapy especially stem cell therapy, on the other hand, has recently met great success as a novel regenerative medicine to support solid organ transplantation including human islet transplantation. Among all types of stem cells, mesenchymal stem cells (MSCs) are given special interest for their self-renewal potential, multi-lineage capacities, paracrine effects (trophic mediator) and immune modulatory effects, making it a great candidate for improving human islet transplantation. MSCs are mostly found in bone marrow, adipose and umbilical cord blood, are one of the most extensively studies adult stem cells used in treating degenerative diseases as well as solid organ transplantation. Unlike embryonic stem cells (ESCs) or induced pluripotent stem cells (iPSCs), adult stem cells show restricted proliferation and lineage differentiation, and consequently have less risk of inducing tumor. MSC-based therapy has been used to improve human islet transplantation from three major aspects. First, MSC was used as an immune modulator to prevent the immune rejection of human islets. Second, MSC was used as a trophic mediator to improve the revascularization of human islets. Third, MSC 
was induced in vitro to form insulin producing cell as an alternative sources of human islets. The third aspect seems to be quite promising theoretically, but no group has successfully generated enough "artificial" islets for human transplantation. Our focus is mainly on the immunomodulatory effects and the trophic effects of MSCs to improve the human islet transplantation.

We also introduce humanized mouse model into the studies of islet transplantation. Humanized mouse model contains mature human immunity and is a good model to determine the immune rejection to human islet after transplantation. Our study is innovative because this is the first time humanized animal is used test the efficacy of MSC based regenerative medicine on solid organ transplantation. We plan to answer the following questions in this study. 1) Can humanized mouse model pose an immune rejection to human islets after transplantation? 2) What is the duration and limitation of the adoptively transferred human immunity? 3) What is the efficacy and mechanism of stem cell therapy in improving human islet transplantation in humanized mouse model? 


\section{TABLE OF CONTENTS}

CHAPTER 1. INTRODUCTION ....................................................................................1

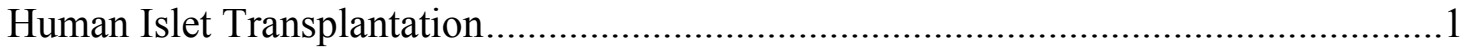

Challenges of Human Islet Transplantation...............................................................

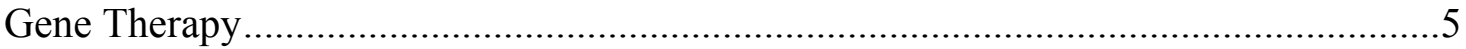

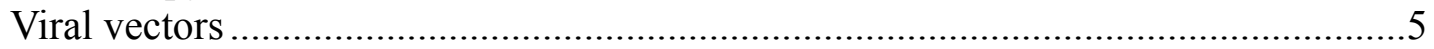

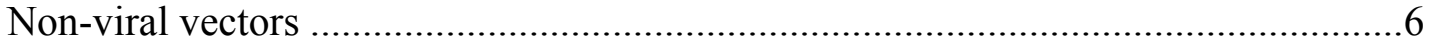

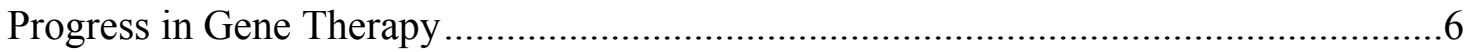

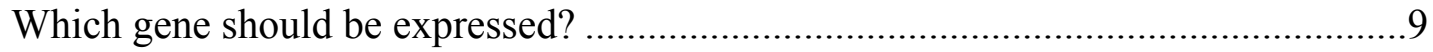

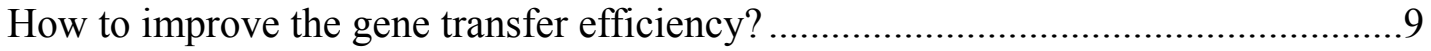

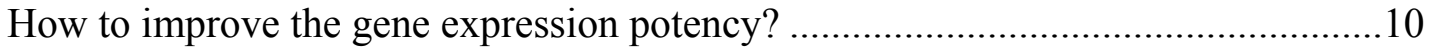

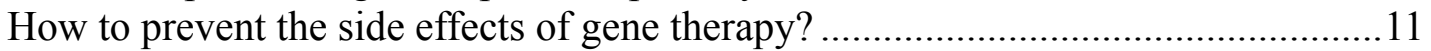

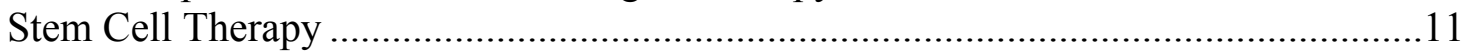

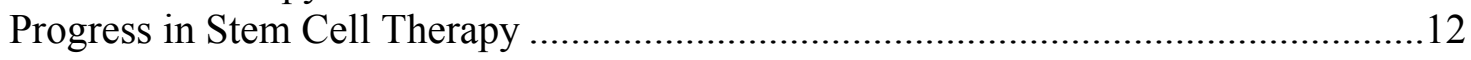

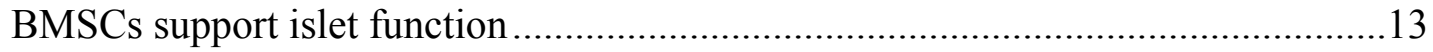

BMSCs prevent graft rejection ................................................................. 14

Stem Cell Based Gene Therapy ............................................................................... 16

Diabetic Humanized Animal Model .......................................................................... 19

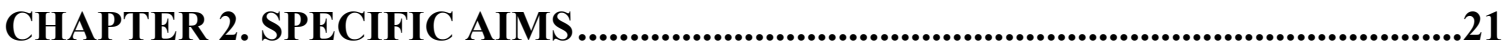

Aim I. Adenovirus Mediated XIAP Expression to Prevent the Early Graft

Apoptosis in Human Islet Transplantation ..................................................................21

Aim II. RGD Peptide Modified Adenovirus Encoding XIAP and HGF to

Simultaneously Prevent Early Graft Apoptosis and Promote Revascularization to Improve the Outcome of Human Islet Transplantation ........................................21

Aim III. Use BMSCs as Gene Delivery Vehicles to Express HGF and IL-1Ra to Improve the Outcome of Human Islet Transplantation ...........................................22

Aim IV. Use BMSCs to Prevent the Immune Rejection of Islet Allografts in

Humanized NSG Mice........................................................................................22

Aim V. Use Small Molecular Compound Drug to Prevent the Immune Rejection of

Islet Allografts in Humanized NSG Mice................................................................23

\section{CHAPTER 3. XIAP OVEREXPRESSON MEDIATED BY ADENOVIRUS TO PREVENT APOPTOSIS OF ISLET TRANSPLANTATION ...............................24}

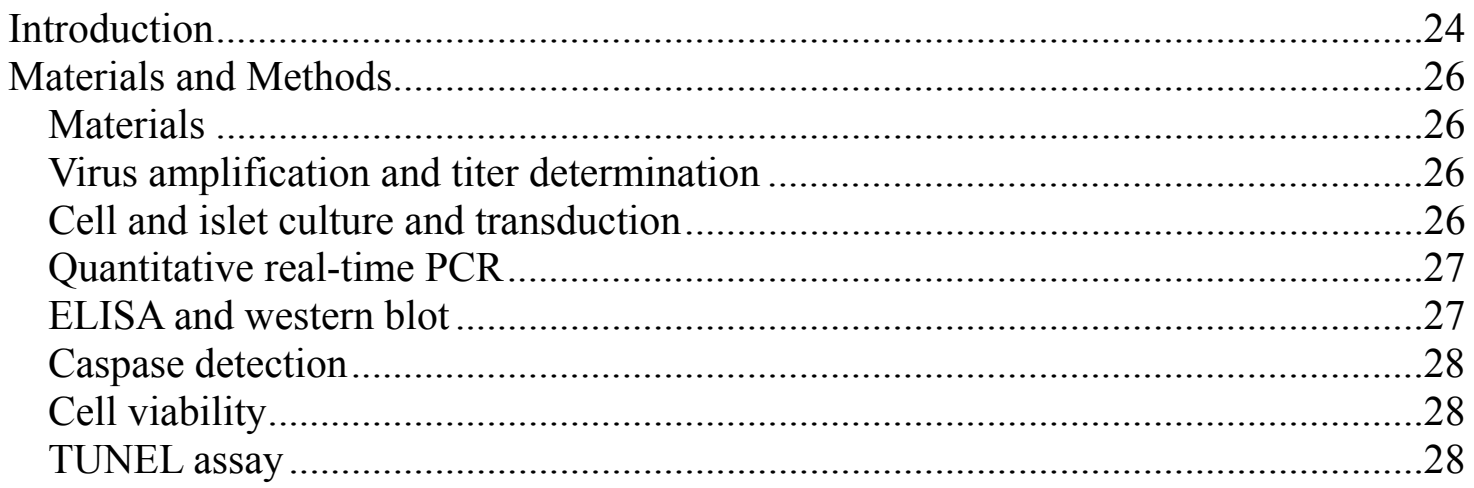




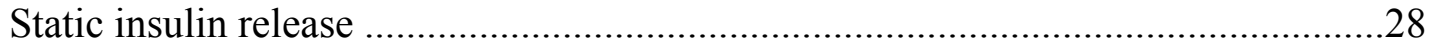

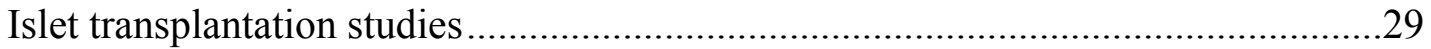

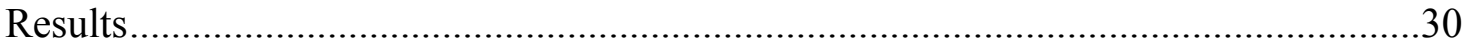

XIAP gene expression in INS-1E cells and human islets ..........................................30

XIAP improves INS-1E cells viability and function against inflammatory

cytokines ……....................................................................................................

XIAP inhibits INS-1E cell apoptosis against inflammatory cytokines .......................30

XIAP inhibits caspase activity in INS-1E cells and human islets................................34

Prolonged normoglycemic control of diabetic mice by Adv-hXIAP transduced

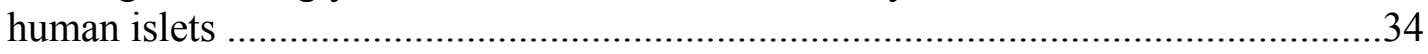

Protective effect of Adv-hXIAP transduction will be reversed by XIAP inhibitor

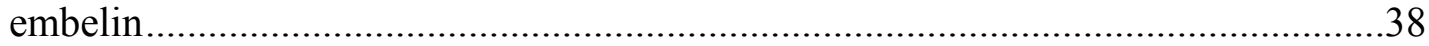

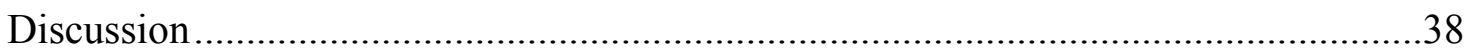

\section{CHAPTER 4. RGD MODIFIED ADENOVIRUS ENCODING XIAP AND HGF TO SIMULTANEOUSLY PREVENT EARLY GRAFT APOPTOSIS AND PROMOTE REVASCULARIZATION TO IMPROVE THE OUTCOME OF HUMAN ISLET TRANSPLANTATION .........................................................................43}

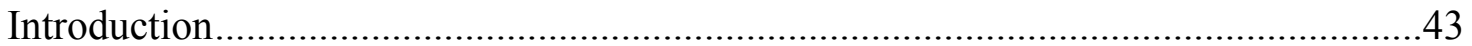

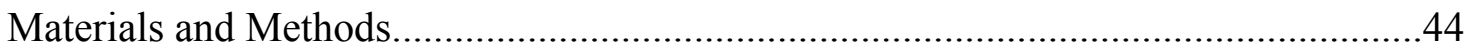

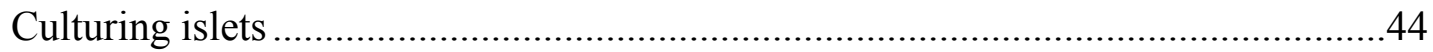

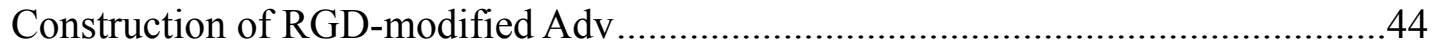

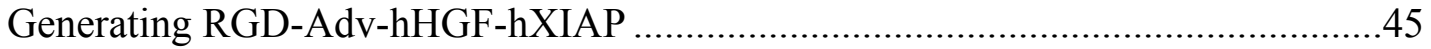

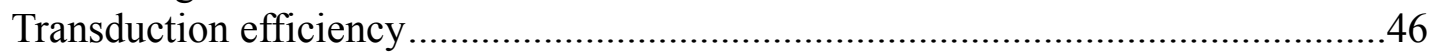

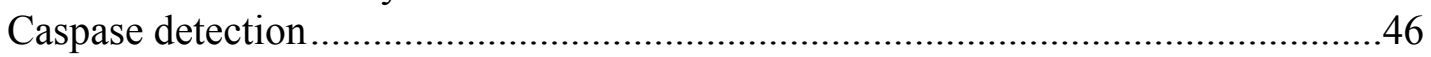

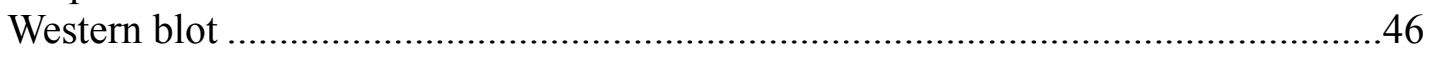

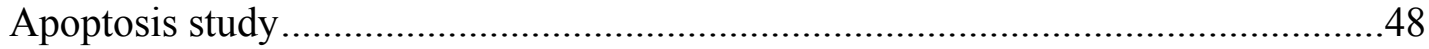

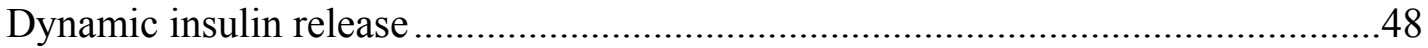

Generate moderate diabetic nod-scid mice ..........................................................48

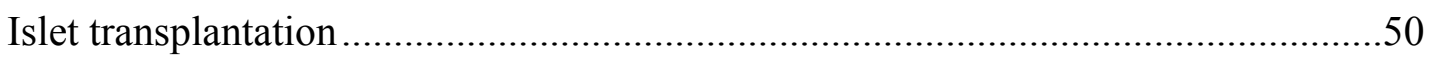

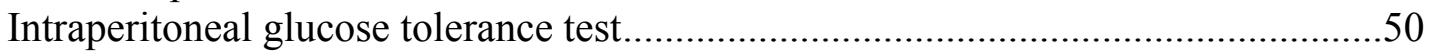

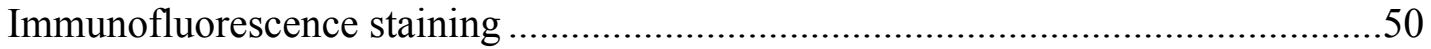

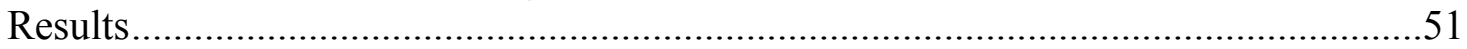

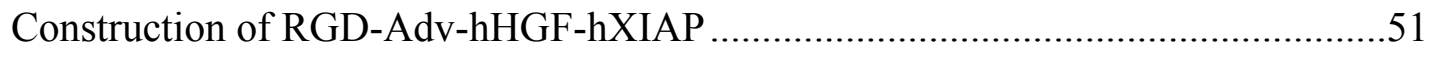

Transduction efficiency of RGD-Adv-hHGF-hXIAP on human islets .....................51

Pro-apoptotic gene inhibited and anti-apoptotic gene elevated ................................53

Protection of RGD-Adv-hHGF-hXIAP on human islets ......................................53

Generation of diabetic NOD-SCID mice for human islet transplantation ..................53

RGD-Adv-hHGF-hXIAP transduction prolonged islet survival after

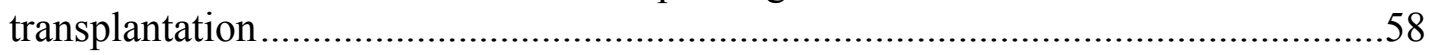

RGD-Adv-hHGF-hXIAP transduction improved islet revascularization after

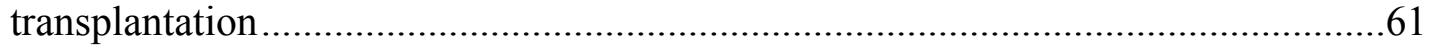

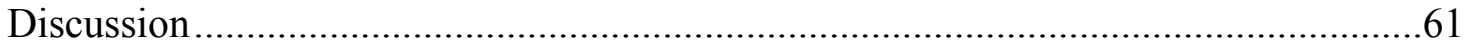




\section{CHAPTER 5. BMSC AS GENE DELIVERY VEHICLE TO EXPRESS HGF AND IL-1RA TO IMPROVE THE OUTCOME OF HUMAN ISLET TRANSPLANTATION}

Introduction .66

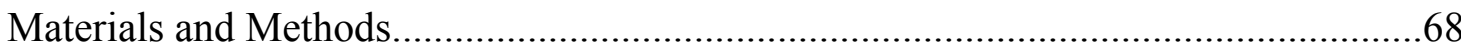

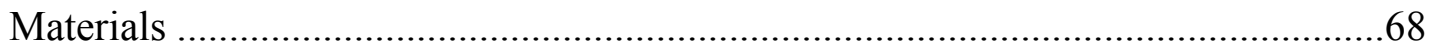

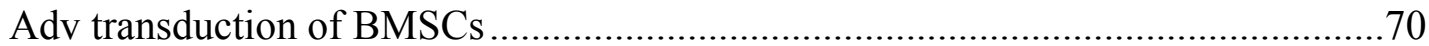

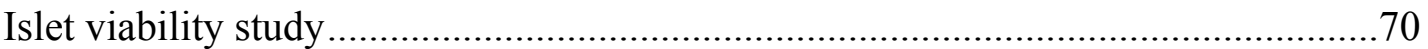

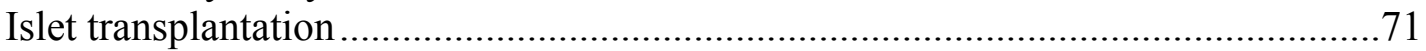

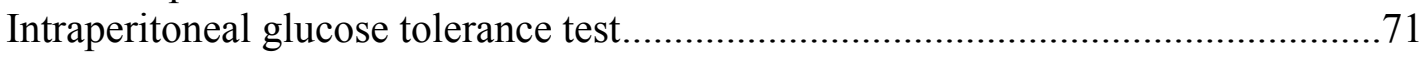

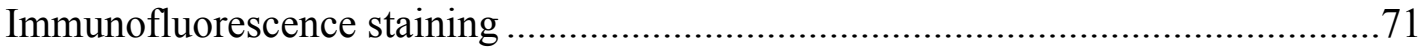

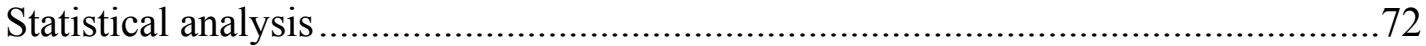

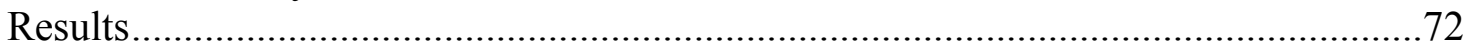

Characterization of Adv-hHGF-hIL-Ra-transduced BMSCs...................................72

Protection of human islets by Adv-hHGF-hIL-Ra-transduced BMSCs ....................72

Co-transplantation of islets with Adv-hHGF-hIL-Ra-transduced BMSCs improved outcome of islet transplantation.......................................................... 74

Adv-hHGF-hIL-Ra-transduced BMSCs promoted islet engraftment and

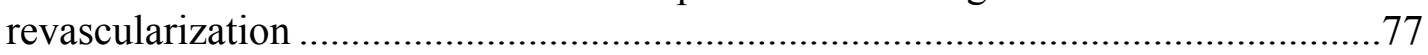

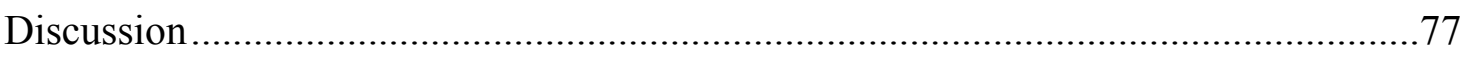

\section{CHAPTER 6. BMSC PREVENTS ALLOGRAFT REJECTION OF HUMAN} ISLET TRANSPLANTATION IN HUMANIZED MOUSE MODEL ......................85

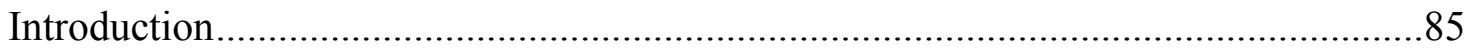

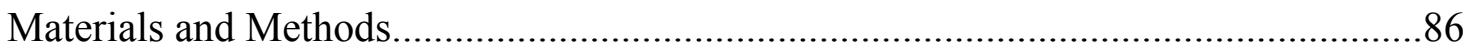

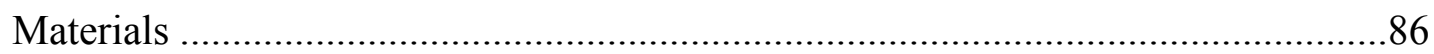

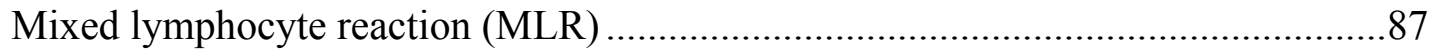

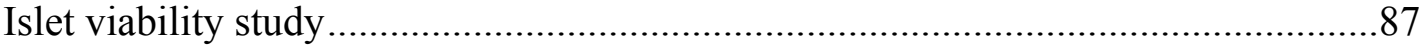

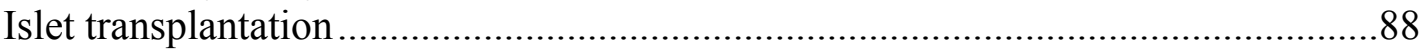

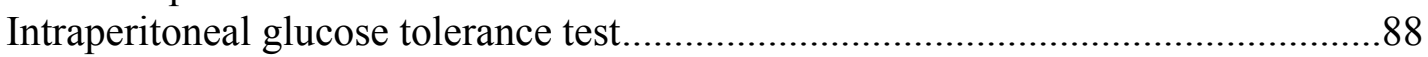

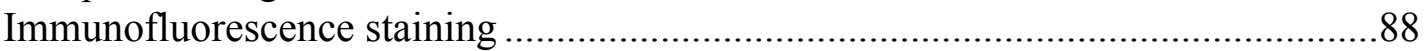

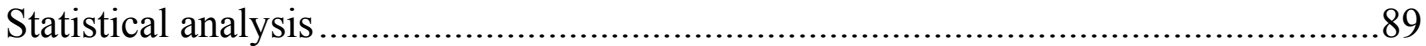

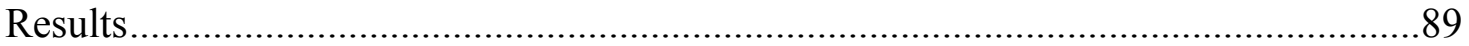

Generation and characterization of humanized NSG mice ...................................89

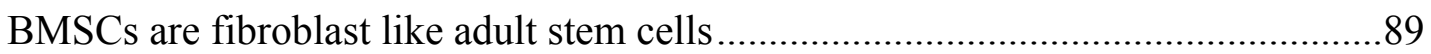

BMSCs suppressed the activation and proliferation of PBMCs .............................89

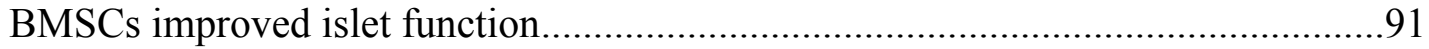

BMSCs improved islet transplantation in humanized NSG mice............................94

BMSCs prevented $\mathrm{T}$ cell infiltration and increased Tregs ....................................98

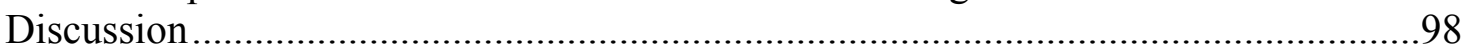

\section{CHAPTER 7. A NOVEL COMPOUND DRUG FOR HUMAN ISLET} TRANSPLANTATION IN HUMANIZED MOUSE MODEL ..................................104

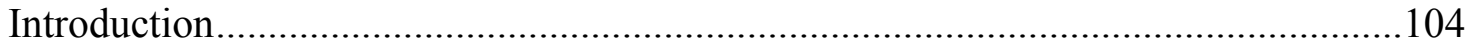

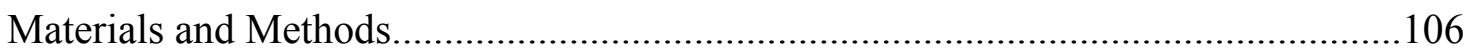




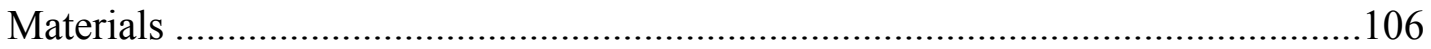

Synthesis and characterization of antiapoptotic immunosuppressive drug ............106

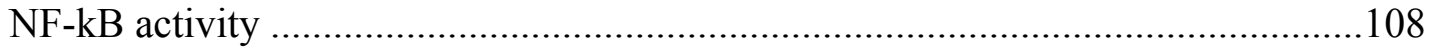

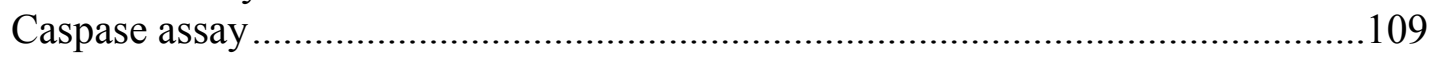

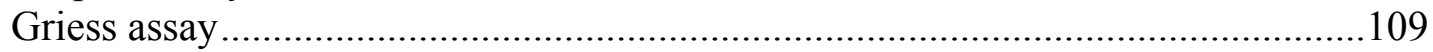

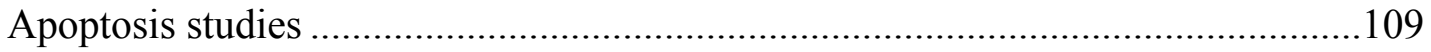

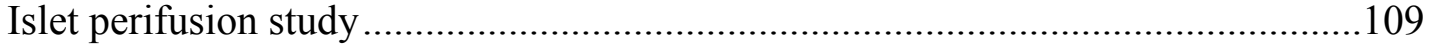

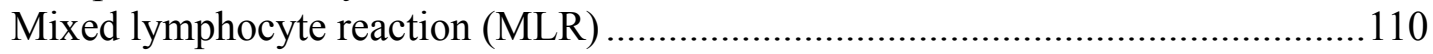

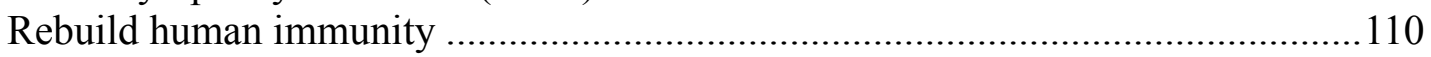

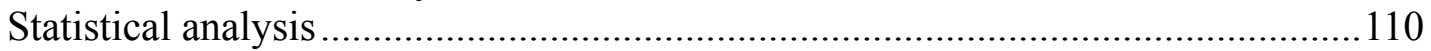

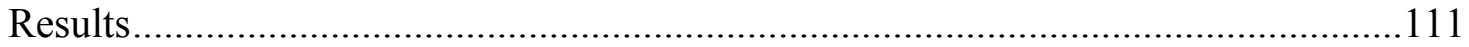

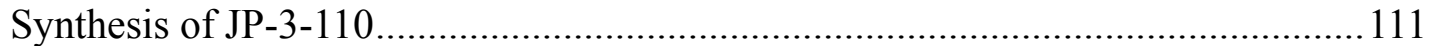

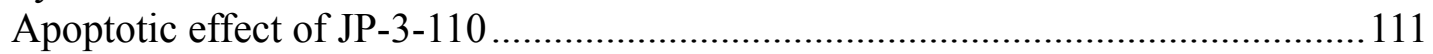

Immunosuppressive effect of JP-3-110 ......................................................... 111

JP-3-110 inhibited the function of T cells and B cells in humanized NSG mice.....117

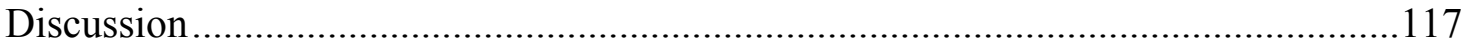

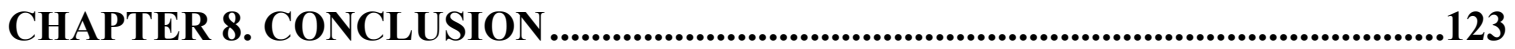

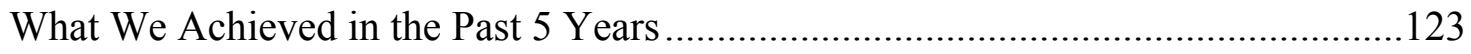

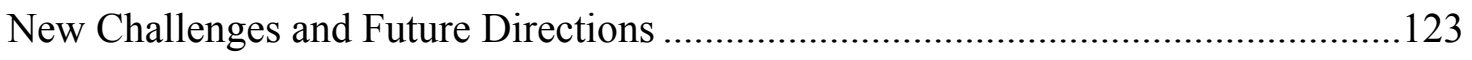

LIST OF REFERENCES ....................................................................................................127

VITA 


\section{LIST OF TABLES}

Table 1-1. Reasons for islet graft failure and useful interventions .............................4

Table 1-2. Characteristics of viral vectors for gene transfer .......................................

Table 1-3. Summary of non-viral methods used for gene transfer..............................

Table 1-4. BMSCs improve solid organ transplantation .........................................17

Table 3-1. Mouse weight measurements after human islet transplantation ..................37

Table 7-1. The pharmacological activities of KZ41 and MPA ….............................120 


\section{LIST OF FIGURES}

Figure 1-1. The process of clinical islet transplantation for the treatment of type 1

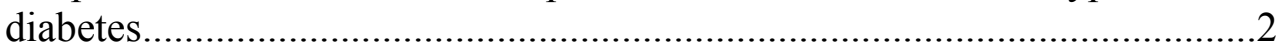

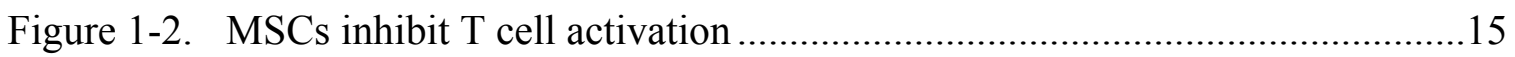

Figure 1-3. Gene therapy and stem cell based gene therapy ……………………….....18

Figure 3-1. Schematic diagram showing the role of XIAP in the apoptotic pathway of human islets 25

Figure 3-2. Adenovirus mediated overexpression of XIAP in INS-1E cells and human islets.

Figure 3-3. Beneficial effect of XIAP overexpression on INS-1E cell viability and function

Figure 3-4. Anti-apoptotic effect of XIAP overexpression on INS-1E cells.

Figure 3-5. XIAP overexpression inhibits caspase activities of INS-1E cells and human islets after cytokine treatment

Figure 3-6. Effect of Adv-hXIAP transduction on the outcome of islet transplantation

Figure 3-7. Immunohistochemical staining of kidney sections at 42 days after transplantation.

Figure 3-8. Beneficial effect of XIAP overexpression on INS-1E cells can be reversed by XIAP inhibitor embelin

Figure 4-1. Construction of RGD modified Adv genome encoding HGF and XIAP in $\mathrm{E} 1$ and $\mathrm{E} 3$ deletion

Figure 4-2. Islet perifusion set for dynamic analysis of function of human islets.

Figure 4-3. Transduction efficiency of RGD-Adv-hHGF-hXIAP into human islets ......52

Figure 4-4. HGF and XIAP expression led to inhibition of pro-apoptotic gene and elevation of anti-apoptotic gene.

Figure 4-5. Transgene expression of HGF and XIAP protected INS-1E cells and human islets from inflammatory cytokine induced apoptotic cell death .....55

Figure 4-6. Effect of HGF and XIAP gene expression on islet function. .56 
Figure 4-7. The manner and dose of STZ injection has great impact on the diabetic level of NOD-SCID mice.

Figure 4-8. Effect of RGD-Adv-hHGF-hXIAP transduction on the outcome of islet transplantation

Figure 4-9. Intraperitoneal glucose tolerance test of the mice at 30 days after islet transplantation.

Figure 4-10. Immunofluorescence staining of the kidney section bearing RGD-AdvhHGF-hXIAP-transduced human islets at 30 days after islet transplantation.....

Figure 4-11. Immunofluorescence staining of the kidney section bearing RGD-AdvhHGF-hXIAP-transduced human islets at 200 days after islet transplantation.

Figure 5-1. A schematic demonstration of Adv-hHGF-hIL-1Ra-transduced BMSCs co-transplantation with human islets.

Figure 5-2. Construction of bipartite Adv-hHGF-hIL-1Ra using AdenoQuick cloning system.

Figure 5-3. Transduction efficiency of Adv-hHGF-hIL-1Ra into BMSCs .73

Figure 5-4. Adv-hHGF-hIL-1Ra-transduced BMSCs helped to preserve islet viability against IL-1 $\beta$ stimulation .75

Figure 5-5. Outcome of islet transplantation after being cotransplanted with AdvhHGF-hIL-1Ra-transduced BMSCs .... .76

Figure 5-6. Body weight and blood glucose after human islet transplantation .78

Figure 5-7. Intraperitoneal glucose tolerance test of the mice receiving 1000 IEs and 1000 IEs co-transplanted with Adv-hHGF-hIL-1Ra-transduced BMSCs at 2 weeks after islet transplantation

Figure 5-8. Immunofluorescence staining of the kidney section bearing islets and Adv-hHGF-hIL-1Ra-transduced BMSCs ....

Figure 5-9. Adv-hHGF-hIL-1Ra-transduced BMSCs promoted revascularization of the transplanted islets

Figure 6-1. Establish human immunity in NSG mice using human PBMCs .90

Figure 6-2. Characterization of human BMSCs .90

Figure 6-3. Human BMSCs prevent the proliferation and activation of PBMCs .92 
Figure 6-4. CD14+ monocytes and IL-10 were required for the immunomodulatory effect of BMSCs

Figure 6-5. Human BMSCs protected the function of human islets against the inflammatory cytokines.

Figure 6-6. The outcome of human islet transplantation with BMSCs in diabetic humanized NSG mice

Figure 6-7. Human BMSCs prevent the $\mathrm{T}$ cell infiltration into the transplantation site .99

Figure 6-8. Schematic illustration of why BMSCs improved human islet transplantation

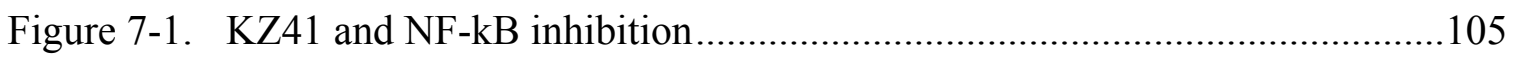

Figure 7-2. Synthesis and characterization of JP-3-110 ………………………........107

Figure 7-3. Use HPLC to determine the solubility of JP-3-110 ..................................112

Figure 7-4. JP-3-110 and KZ41 suppressed NF-kB activity in INS-1E cells................113

Figure 7-5. KZ41 and JP-3-110 pretreated INS-1E cells were resistant to cytokine induced apoptotic cell death.

Figure 7-6. KZ41 inhibited the apoptotic cell death of human islets after stimulation with cytokine cocktail

Figure 7-7. JP-3-110 suppressed $\mathrm{T}$ cell proliferation but not $\mathrm{T}$ cell activation 116

Figure 7-8. JP-3-110 inhibited the function of T cells and B cells in humanized NSG mice

Figure 7-9. The hydrolysis of JP-3-110 in aqueous solution 122

Figure 8-1. An ideal application of targeting exosomes for islet transplantation in humanized NSG mice 


\section{LIST OF ABBREVIATIONS}

\begin{tabular}{|c|c|}
\hline AAV & Adeno-associated virus \\
\hline Adv & Adenovirus \\
\hline BMSC & Bone marrow derived mesenchymal stem cell \\
\hline CFSE & Carboxyfluorescein succinimidyl ester \\
\hline CMV & Cytomegalovirus \\
\hline DAB & 3,3 diaminobenzidine tetrahydrochloride \\
\hline DAPI & 4',6-diamidino-2-phenylindole \\
\hline DC & Dendritic cell \\
\hline ELISA & Enzyme-linked immunosorbent assay \\
\hline ESC & Embryonic stem cell \\
\hline FBS & Fetal bovine serum \\
\hline GvHD & Graft versus host disease \\
\hline HGF & Hepatocyte growth factor \\
\hline HRP & Horse radish peroxidase \\
\hline IE & Islet equivalent \\
\hline $\mathrm{IFN} \gamma$ & Interferon $\gamma$ \\
\hline IL-1 $\beta$ & Interleukin $1 \beta$ \\
\hline IL-1Ra & Interleukin 1 receptor antagonist \\
\hline IL-2 & Interleukin 2 \\
\hline IL-2R & Interleukin 2 receptor \\
\hline IL-10 & Interleukin 10 \\
\hline IL-10sR & Interleukin 10 soluble receptor \\
\hline INS-1E & Rat insulinoma cells \\
\hline iPSC & induced pluripotent stem cell \\
\hline JP-3-110 & $\begin{array}{l}\text { (E)-2,3,5-trihydroxy-5-(propylcarbamoyl)cyclohexyl 6-(4-ethoxy- } \\
\text { 6-methoxy-7-methyl-3-oxo-1,3-dihydroisobenzofuran-5-yl)-4- } \\
\text { methylhex-4-enoate }\end{array}$ \\
\hline KZ41 & 1,3,4,5-tetrahydroxy-N-propylcyclohexanecarboxamide \\
\hline MLR & Mixed lymphocyte reaction \\
\hline MOI & Multiplicity of infection \\
\hline MPA & Mycophenolic acid \\
\hline MSC & Mesenchymal stem cell \\
\hline NO & Nitric oxide \\
\hline NOD-SCID & Nonobese diabetic severe combined immune deficient \\
\hline NSG & NOD-SCID gamma or NOD.Cg-Prkdc ${ }^{\text {scid }}{ }^{1} 2 \mathrm{rg}^{\mathrm{t}{ }^{1} \mathrm{Wjl}} / \mathrm{SzJ}$ \\
\hline Pax4 & Plasmid vectors encoding paired box gene 4 \\
\hline PBMC & Peripheral blood mononuclear cell \\
\hline PBS & Phosphate buffer solution \\
\hline Pdx1 & Pancreatic duodenal homeobox 1 \\
\hline PGE2 & Prostaglandin E2 \\
\hline PNF & Primary nonfunction \\
\hline QA & Quinic acid \\
\hline RES & Reticulo-endothelial system \\
\hline
\end{tabular}




$\begin{array}{ll}\text { ROS } & \text { Reactive oxygen species } \\ \text { SCF } & \text { Stem cell factor } \\ \text { SEAP } & \text { Secreted alkaline phosphatase } \\ \text { shRNA } & \text { Short hairpin RNA } \\ \text { siRNA } & \text { Small interference RNA } \\ \text { STZ } & \text { Streptozotocin } \\ \text { Tc } & \text { Cytotoxic T cell } \\ \text { TEM } & \text { Transmission electronics microscope } \\ \text { TERT } & \text { Telomerase reverse transcriptase } \\ \text { TGF } & \text { Transforming growth factor } \\ \text { Th } & \text { Helper T cell } \\ \text { TNF } \alpha & \text { Tumor necrotic factor } \alpha \\ \text { Treg } & \text { Regulatory T cell } \\ \text { TUNEL } & \text { Terminal deoxynucleotidyl transferase dUTP nick end labeling } \\ \text { UV } & \text { Ultraviolet } \\ \text { VEGF } & \text { Vascular endothelia growth factor } \\ \text { vWF } & \text { von-Willebrand factor } \\ \text { XIAP } & \text { X-linked inhibitor of apoptosis }\end{array}$




\section{CHAPTER 1. INTRODUCTION*}

\section{Human Islet Transplantation}

Diabetes is affecting 25.8 million people in United States. Among them, 5\% are type 1 diabetes or insulin dependent diabetes. Edmonton Protocol for human islet transplantation, first introduced by Dr. Shapiro and his group from University of Alberta in late 1990s [1], has the potential to be a permanent treatment for patients with type 1 diabetes.

Edmonton Protocol described a manner to isolate islets using a mixture of highly purified enzymes from the pancreas of a deceased donor (Figure 1-1) [2]. Collagenase solution is injected into the pancreatic duct, runs through the whole pancreas and causes distension of the pancreas. Pancreas is subsequently cut into small chunks and subjected to further digestion until the islets are liberated and removed from the solution. Isolated islets are then separated from the exocrine tissue and debris in a process called purification. During the transplant, transplant surgeons are guided by ultrasound and radiography to place a catheter through the upper abdomen and into the portal vein of the liver. The islets are then infused through the catheter into the liver. Each recipient receives islets from one to as many as three donors. The islets are infused into the patient's portal vein, and are then kept from being destroyed by the recipient's immune system through the use of two immunosuppressants, sirolimus and tacrolimus as well as a monoclonal antibody drug used in transplant patients called daclizumab. The blood glucose of the patient will be closely monitored and insulin may be needed until control is achieved. If the transplantation outcome is not good, a second infusion of islets may be needed.

\section{Challenges of Human Islet Transplantation}

In the last two decades, human islet transplantation has marched a long trip from bench to bedside as a potential treatment for type 1 diabetes. According to the 2010 annual report conducted by Collaborative Islet Transplant Registry which is funded by the National Institute of Diabetes and Digestive and Kidney Diseases, 571 patients with type 1 diabetes have received human islet transplantation worldwide and nearly twothirds of recipients achieved "insulin independence", a status defined as being able to stop insulin injections for at least 14 days, during the year following transplantation. However,

\footnotetext{
* Portions modified with permission from American Chemical Society. Narang AS and Mahato RI (2006) Biological and biomaterial approaches for improved islet transplantation. Pharmacol Rev 58: 194-243. Other portions modified with permission from Springer. Wu H, Ye Z and Mahato RI (2011) Genetically Modified Mesenchymal Stem Cells for Improved Islet Transplantation. Mol. Pharmaceutics 8: 1458-1470.
} 


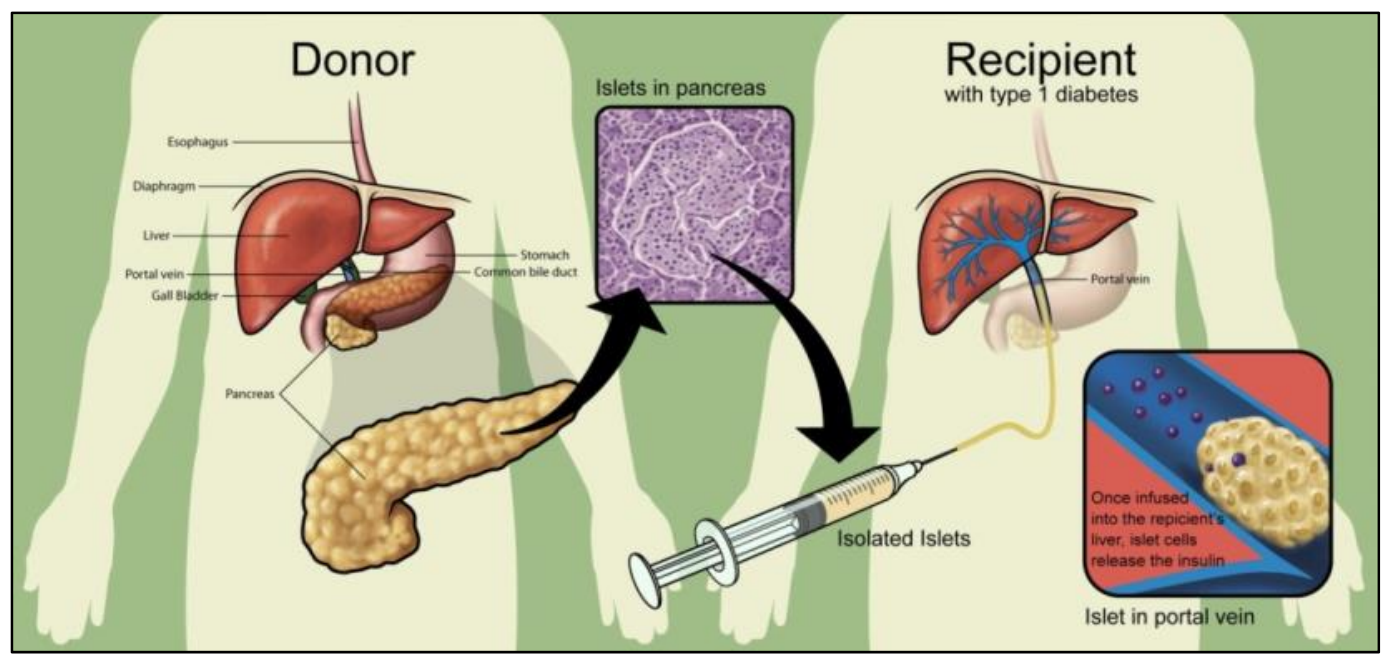

Figure 1-1. The process of clinical islet transplantation for the treatment of type 1 diabetes 
insulin independence is difficult to maintain over time. At 2-year follow-up, the proportion dropped to about one-third of recipients and at 5-year follow-up, the proportion dropped to below 20\%. This fact explained why less North American centers performed islet transplantation and Edmonton Protocol is still an experimental treatment for type 1 diabetes. Two of the most important limitations to the widespread of human islet transplantation are the limited supply of islets for transplantation and the inadequate means for preventing islet loss after transplantation [2]. Moreover, because of the extensive posttransplantation challenges, a patient needs at least 10,000 islet equivalents (IEs) per kilogram of body weight (extracted from two or more donor pancreases) for an optimal transplantation outcome, making the current shortage in islet supply even worse $[3,6]$. The major reasons for islet graft failure and useful interventions are listed in Table 1-1.

To address the issue of limited supply of human islets, researchers are seeking alternative sources of human islets by either using islets from alternative species or generating human islets and insulin-producing cells from stem cells. Porcine islets are widely reported as a competent alternative for xenogeneic islet transplantation with the assistance of biological and biomaterial approaches to prevent enhanced immune destruction of the xenografts $[7,8]$. For in vitro transdifferentiation strategy, several groups have reported successful generation of insulin-producing $\beta$-cells and islet-like structures from both ES cells. Lumelsky, et al. demonstrated that ES cells could differentiate in to insulin-producing cells which self-assemble into islet-like clusters.[9] Blyszczuk et al. reported the differentiation of ES cells into insulin-producing cells through transduction of plasmid vectors encoding paired box gene 4 (Pax-4) and pancreatic duodenal homeobox 1 (Pdx-1).[10] However, caution should be exercised as the differentiation from pluripotent ES cells can not 100\% and the remaining undifferentiated ES cells may still hold tumorigenicity. Several groups used MSCs as a relatively safer source and succeed in generation islet-like cluster or insulin-producing cells [11-13]. However, most of them relied on genetic manipulation of MSCs and the ability of producing large numbers of functional tissues by this means was not proven.

Islet loss happens because of two major reasons, the immune rejection from the recipients and the primary nonfunction (PNF) of islet grafts [2]. Immune rejection describes a process where transplanted islets are attached, recognized and attacked by the host immune system. Immunosuppressive regimens are capable of preventing immune rejection from months to years, but the agents used in these treatments may induce significant side effects, resulting in progressive decline in graft function. Some of the most commonly used immunosuppressive agents such as tacrolimus (also known as FK506 or Fujimycin), mycophenolic acid (MPA), and sirolimus (also known as rapamycin) are also deleterious to islet function and insulin secretion [4, 5]. PNF is characterized as the loss of islet viability and function caused by non-immune reactions, such as the disruption of islet microvasculature during islet isolation and purification process, hypoxia in the core of islet grafts and production of inflammatory cytokines at the transplantation sites. Once PNF happens, islets are still alive but in an apoptosis process and ceasing to actively produce insulin in response to the fluctuation of the blood glucose level. Failure to revascularization and subsequent apoptosis are the most frequent reasons 
Table 1-1. Reasons for islet graft failure and useful interventions

\begin{tabular}{ll}
\hline $\begin{array}{l}\text { Obstacles to islet } \\
\text { transplantation }\end{array}$ & Strategies \\
\hline $\begin{array}{l}\text { Loss of viability during } \\
\text { isolation }\end{array}$ & $\begin{array}{l}\text { Improved enzymatic blends for islet isolation, } \\
\text { culture media composition and conditions }\end{array}$ \\
Failure to revascularize & $\begin{array}{l}\text { Encapsulation with vascular growth promoting } \\
\text { proteins; ex vivo growth factor gene } \\
\text { delivery for revascularization }\end{array}$ \\
$\begin{array}{l}\text { Inflammatory response/immune } \\
\text { rejection }\end{array}$ & $\begin{array}{l}\text { Immunoisolation membranes; generalized } \\
\text { immunosuppression; immune tolerance induction; } \\
\text { antiapoptotic and immune modulating gene } \\
\text { delivery }\end{array}$ \\
Inadequate islet mass & Optimization of islet mass and transplantation site \\
Inadequate islet supply & $\begin{array}{l}\text { Xenotransplantation } \\
\text { Stem cell-based approaches }\end{array}$ \\
\hline
\end{tabular}


leading to PNF. Human islet, in its native status, is a cluster of heterogeneous cell types with extensive intra-islet vasculature formed of fenestrated capillary endothelial lining, which gets disrupted during islet isolation, leading to collapse of vasculature, accumulation of endothelial fragments and compromised perfusion in the core of the islets.[14] Therefore, unlike whole pancreas and other solid organ transplantations, islet transplantation is ectopic and requires extensive and functional revascularization to promote the posttransplantation survival of islet grafts. $[15,16]$ Less or abnormal revascularization usually lead to hypoxia, apoptotic islet death and thus compromised transplantation outcome.

To summarize, the long term outcome of human islet transplantation was impeded by several obstacles, the limited supplies of donor islets, the inadequate means of immune suppression, the loss of islets in the isolation, transportation and transplantation process and the poor post-transplantation revascularization. All these obstacles can be approached from two directions, to increase the resistance of islets or to reduce the immune rejection from the recipients. Gene therapy has been proven to be an effective strategy for achieve the formal goal while stem cell therapy can be used for the latter.

\section{Gene Therapy}

Almost all human diseases are the results of dysfunction or malproduction of proteins. Traditional small molecule drugs usually interact with proteins such as enzymes, hormones and transcriptional factors to exert their therapeutic potential. However, many severe and deliberating diseases (e.g., diabetes, hemophilia, cystic fibrosis) and several chronic diseases (e.g., hypertension, ischemic heart disease, asthma, Parkinson's disease, motor neuron disease, multiple sclerosis) remain inadequately treated by the conventional pharmaceutical approaches.

Gene therapy is the use of nucleic acids as a pharmaceutical agent to treat disease. It derives its name from the idea that DNA can be used to supplement or alter genes within an individual's cells as a therapy to treat disease. Unlike small molecule drugs or protein drugs which are usually formulated in capsule or tablet forms, therapeutic nucleic acids are packaged within a specialized vector to get inside cells within the body.

\section{Viral vectors}

All viruses hold the inherited advantage to bind to their hosts and introduce their genetic material into the host cell with high efficiency. Native viruses must be modified to transfer genetic materials. To construct a viral vector, the genes responsible for the viral replication and pathogenicity are first removed and replaced with a transgene cassette. Then the recombinant viral genome is inserted into a shuttle plasmid and transduced into a packaging cell line which contains the genes responsible for the viral replication to generate the recombinant viral vectors. Viral vectors typically hold high transduction efficiency and do not need additional carriers for effective gene delivery. To 
date approximately $70 \%$ of all gene therapy clinical trials employ viral vectors. Retrovirus, lentivirus, adenovirus and adeno-associated virus (AAV) are the most extensively studied and used viral vectors for human gene therapy. Their characteristics have been listed and compared in Table 1-2.

\section{Non-viral vectors}

The inherited problems with recombinant viruses such as immunogenicity, neutralizing antibodies and insertional mutagenesis have called for the design of efficient non-viral vectors for human gene therapy. Non-viral vectors are significantly less immunogenic and are not likely to induce insertional mutagenesis and unwanted homologous recombination after uptake by the cells. They are also relatively easy to be manipulated, produced and purified in large scale compared with their viral counterparts. Non-viral gene therapy includes local administration of naked plasmid or using specialized carriers such as cationic liposomes and polymer-based systems to deliver plasmids to targeted area (Table 1-3). However, their clinical utility is still hampered by the low transfection efficiency, which stems from non-specific uptake of the vector by the epithelial barriers and extracellular matrix and poor delivery into the therapeutic target. The intracellular gene silencing machinery also prevents the long term transgene expression. New emerging delivery systems and vector-constructing technologies try to address these issues.

Gene therapy usually targets one or more defected genes without affecting the normal gene at the site of diseases. Gene therapy target can be an abnormal oncogene whose product has the potential to cause a tumor or a defect gene whose product is critical to maintain normal physiological functions. Compared with small molecule drugs, gene therapy will not induce drug resistance even after repeated treatments since the targets of gene medicine are not certain receptors but the genes encoding them. Moreover, this technique could be a permanent treatment to give someone that is born with a genetic disease a chance to live a normal life once and for all. However, there are certain limitations of gene therapy as well. The major disadvantage of gene therapy is that gene medicines are not easy to be formulated into conventional dosage forms and delivered for a routine use. Therefore, the clinical applications of gene therapy can so far only be conducted in hospitals with well-trained specialists. The cost of gene therapy is so far also much higher compared with traditional medicines.

\section{Progress in Gene Therapy}

The ultimate goal of gene therapy is to improve the resistance and function of human islets under the challenges after transplantation. Four important questions should be asked for the success of gene therapy on human islet transplantation. Which gene should be expressed? How to improve the gene transfer efficiency? How to improve the gene expression potency? How to prevent the side effects of gene therapy? 
Table 1-2. Characteristics of viral vectors for gene transfer

\begin{tabular}{|c|c|c|c|c|}
\hline Characteristics & Retrovirus & Lentivirus & Adenovirus & Adeno-associated virus \\
\hline Genetic material & RNA & RNA & dsDNA & ssDNA \\
\hline Genome size & $7-11 \mathrm{~kb}$ & $8 \mathrm{~kb}$ & $26-45 \mathrm{~kb}$ & $4.7 \mathrm{~kb}$ \\
\hline Cloning capacity & $8 \mathrm{~kb}$ & $8 \mathrm{~kb}$ & $7-35 \mathrm{~kb}$ & $<5 \mathrm{~kb}$ \\
\hline Genome forms & Integrated & Integrated & Episomal & Stable/Episomal \\
\hline Diameter & $100-145 \mathrm{~nm}$ & $80-120 \mathrm{~nm}$ & $80-100 \mathrm{~nm}$ & $20-22 \mathrm{~nm}$ \\
\hline Tropism & Dividing cells only & $\begin{array}{l}\text { Broad, Dividing \& Non- } \\
\text { dividing cells }\end{array}$ & $\begin{array}{l}\text { Broad, Dividing \& Non- } \\
\text { dividing cells }\end{array}$ & $\begin{array}{l}\text { Broad, not suitable for } \\
\text { hematopoietic cells }\end{array}$ \\
\hline Virus protein expression & No & Yes/No & Yes/No & No \\
\hline Transgene expression & Slow, constitutive & Slow, constitutive & $\begin{array}{l}\text { Rapid, } \\
\text { Transient }\end{array}$ & $\begin{array}{l}\text { Moderate, constitutive, } \\
\text { transient }\end{array}$ \\
\hline Delivery method & Ex vivo & Ex vivo & Ex/In vivo & Ex/In vivo \\
\hline $\begin{array}{l}\text { Typical yield } \\
\text { (viral particle/ml) }\end{array}$ & $<10^{8}$ & $<10^{7}$ & $<10^{14}$ & $<10^{13}$ \\
\hline Pre-existing immunity & Unlikely & $\begin{array}{l}\text { Perhaps, } \\
\text { Post-entry }\end{array}$ & Yes & Yes \\
\hline Immunogenicity & Low & Low & High & Moderate \\
\hline Potential pathogenicity & Low & High & Low & None \\
\hline Safety & Insertional Mutagenesis & Insertional Mutagenesis & $\begin{array}{l}\text { Potent Inflammatory } \\
\text { Response }\end{array}$ & $\begin{array}{l}\text { None to date but long- } \\
\text { term not clear. }\end{array}$ \\
\hline Physical stability & Poor & Poor & Fair & High \\
\hline
\end{tabular}


Table 1-3. Summary of non-viral methods used for gene transfer

\begin{tabular}{|c|c|c|}
\hline Methods & Advantages & Disadvantages \\
\hline Naked DNA & No special skills needed, easy to produce. & $\begin{array}{l}\text { Low transduction efficiency, transient gene } \\
\text { expression. }\end{array}$ \\
\hline Microinjection & $\begin{array}{l}\text { Up to } 100 \% \text { transduction efficiency (nuclear } \\
\text { injection) }\end{array}$ & $\begin{array}{l}\text { Requires highly specialized skills for delivery, } \\
\text { limited to ex vivo delivery. }\end{array}$ \\
\hline Gene gun & $\begin{array}{l}\text { Easy to perform, effective immunization with low } \\
\text { amount of DNA. }\end{array}$ & Poor tissue penetration. \\
\hline Electroporation & High transduction efficiency. & $\begin{array}{l}\text { Transient gene expression, toxicity, tissue damage, } \\
\text { highly invasive. }\end{array}$ \\
\hline Sonoporation & Method well tolerated for other applications. & $\begin{array}{l}\text { Transient gene expression, toxicity not yet } \\
\text { established. }\end{array}$ \\
\hline Laser irradiation & Can achieve $100 \%$ transduction efficiency. & Special skills and expensive equipment necessary. \\
\hline Magentofection & Safety of method established in the clinic. & Poor efficiency with naked DNA. \\
\hline Liposomes & Easy to produce. & $\begin{array}{l}\text { Protein and tissue binding, transient gene } \\
\text { expression. }\end{array}$ \\
\hline Micelles & Easy to produce and manipulate. & Unstable, protein and tissue binding. \\
\hline Cationic polymers & $\begin{array}{l}\text { High DNA loading, easy to produce and } \\
\text { manipulate. }\end{array}$ & Transient gene expression, toxicity. \\
\hline Dendrimers & High DNA loading, high transduction efficiency. & Extremely toxic. \\
\hline Solid lipid nanoparticles & Low Toxicity, controlled release and targeting. & NA \\
\hline
\end{tabular}




\section{Which gene should be expressed?}

Numerous genes have been introduced into human islets to prevent the loss of function and viability of islets after transplantation. Most of these genes can be attributed into two categories, the genes preventing the apoptosis of islets and the genes promoting the revascularization of islets. In some rare cases, genes regulating insulin production are also introduced into human islets.

The production of inflammatory cytokines especially interleukin-1 $\beta$ (IL-1 $\beta$ ) has been shown to inhibit islet beta cell function as well as to activate islet apoptosis in a nitric oxide-caspase-dependent manner. Gene therapy strategies have been studied to inhibit this pathway. For example, Giannoukakis et al. first reported that adenoviral gene transfer of interleukin 1 receptor antagonist (IL-1Ra) prevented IL-1 $\beta$-mediated nitric oxide production from human islets in vitro as well as the suppression of insulinproducing $\beta$ cell function as determined by glucose-stimulated insulin production [3]. In our lab, Narang et al. and Panakanti et al. combined the gene expression of vascular endothelia growth factor (VEGF) and IL-1Ra to improve the therapeutic effects of single gene therapy while $\mathrm{Li}$ et al. and Cheng et al. targeted downstream pro-apoptotic factors of IL-1 $\beta$ pathway and used small heparin RNA to inhibit the expression of iNOS and caspase 3, respectively [4-7]. During the time we worked on the apoptosis of human islets, we identified a potent anti-apoptotic factor X-linked inhibitor of apoptosis (XIAP), which binds to and inhibits caspase 3, 7 and 9 at the same time. We hypothesized that overexpression of XIAP can be a more effective strategy to improve the resistance of human islets because XIAP showed ubiquitous silencing effects to almost all caspases while previous strategies only targeted one gene at a time. The detailed results are presented in Chapter 3.

Timely and functional revascularization is required for the survival of human islets after transplantation. Gene transfer of angiogenesis factors such as VEGF and hepatocyte growth factor (HGF) can be an effective strategy to fulfill this purpose and has been widely reported by us and other groups [7-9]. VEGF is a popular angiogenic factor, but its effect is so potent that caution should be exercised to avoid aberrant angiogenesis [10]. Recently, HGF has been seen as a more favorable factor because it can prevent apoptotic islet death and can promote the proliferation of pancreatic $\beta$-cells [11].

\section{How to improve the gene transfer efficiency?}

Islet is a compact cluster of about 1000 non-dividing cells. It is difficult to transfect intact islets using non-viral approaches due to low transfection efficiency. Cationic liposomes and polymer-based systems were employed to assist the transfection process but are toxic at high doses $[12,13]$. On the other hand, viral vectors have demonstrated to be effective gene carriers to human islets. For example, replication deficient adenoviral vectors, from which the viral genome E1 and E3 are deleted, are known to efficiently transduce islets and promote transgene expression up to two weeks [14]. In addition, adenovirus vectors can be produced in high titers and there is no risk of 
insertional mutagenesis as they do not integrate into host genome. We and others have demonstrated adenoviral vector-based gene therapy to be effective in promoting the revascularization and engraftment of human islets posttransplantation $[12,15]$. For example, growth factor gene expression can significantly improve the islet revascularization after transplantation $[4,8,12]$, while anti-apoptotic protein expression can increase the resistance of islet grafts to multiple posttransplantation challenges in human islets $[5,6,15]$. The recent success in constructing bipartite viral vectors which can simultaneously promote revascularization and increase the resistance of transplanted islets may highlight the promising future of gene therapy [4, 8]. However, because of the cluster-like property of islet, multiplicity of infection (MOI) of higher than 500 is usually required to achieve optimal transduction efficiency as suggested from many reports $[4,6$, $8,16]$. Therefore, despite its effectiveness, the clinical application of gene therapy is still hindered by the high risk of immunogenicity of viral vectors.

\section{How to improve the gene expression potency?}

Gene expression potency involves two aspects, gene expression duration and gene expression level. Gene expression duration is usually determined by the choices of vectors while gene expression level is usually determined by the structure of vectors.

Previous studies suggested that the first two weeks is of critical important to the success of the whole islet transplantation because most of the islet death or loss of function happen in that time. Therefore, gene therapy must be able to generate therapeutic proteins in human islets for no shorter than two weeks after gene transfer. Plasmid has been demonstrated to be incapable to express therapeutic proteins for more than 1 week, therefore unsuitable to promise the long term survival of human islets [13, 17]. Adenoviral vectors are the most frequently used gene transfer carriers for human islet transplantation because 1) adenoviruses lead to a potent gene expression to $\sim 2$ weeks which is exactly what islet transplantation requires; 2) adenoviruses can be generated in a higher titer and is easy to be manipulated and modified and 3) adenoviruses causes less risk of insertional mutagenesis and subsequent tumorigenicity. Lentiviral vectors or retroviral vectors have recently attracted some attentions because these vectors promoted transgene expression up to months. But since these vectors will insert genetic materials into host genome, precautions must be taken to avoid the risk of insertional mutagenesis and consequent tumorigenicity.

Structure of vectors, especially the choice of promoters and enhancers, is critical to the expression of therapeutic proteins. Although cytomegalovirus (CMV) promoter drives considerable transgene expression in human islets, the discoveries of new isletspecific promotes and enhancers boost the expression of therapeutic proteins significantly. For example, we previously reported that U6 promoter is transcriptionally more effective than $\mathrm{H} 1$ promoter in human islets [6]. Sharma et al. demonstrated that transgene expression can be drastically increased by incorporating an islets-specific enhance into the construct [18]. Other factors such as the spacing within the construct may also play a role. For example, we found out that insertion of a VEGF expression 
cassette between the CMV promoter and iNOS-shRNA significantly enhanced iNOS gene silencing [19]. Since a majority of my work was done using adenovirus vectors containing CMV promoter which drives optimal transgene expression in both human islets and stem cells. The screening for new islet-specific promotes and enhancers is the not the main scope of my work.

\section{How to prevent the side effects of gene therapy?}

The conflict between the potency and side effects of adenovirus mediated gene therapy is in fact the conflicts between the transduction efficiency and the safety profile, which is further exaggerated by the cluster-like nature of human islets. MOIs higher than 500 are usually required to achieve optimal transduction efficiency but even at this MOI, the cells in the core of islet are hardly transduced. Further increase of MOI will cause toxicity to the cells on the surface of human islets. Another problem with adenovirus mediated gene therapy is that some proteins in the viral genome will be co-expressed with therapeutic proteins though in a lesser extent. This phenomenon may lead to an unwanted outcome; human islets transduced with adenovirus will be recognized and cleared by the host immune system in a significantly faster manner.

To solve the side effects of adenovirus without significantly impaired its transduction efficiency, modified adenovirus was proposed. Adenovirus is usually modified in two manners, which are to reduce the immunogenicity or to increase the transduction efficiency. For the formal purpose, more viral genome was deleted or replaced with transgene to reduce the immunogenicity of adenovirus. The first generation of adenovirus contained only E1 deletion. Recently adenoviruses with both E1 and E3 inserts to simultaneously express two therapeutic genes have been reported [8]. Moreover, adenoviruses with E1, E3 and E4 deletion and even "gutless" adenovirus have been constructed to drive transgene expression [20,21]. For the latter purpose, researchers have been modifying the genome of adenovirus in such a way that some transduction-assisting ligands can be expressed on the surface of the viral particles. Arginine-Glycine-Aspartic acid (RGD) peptide is the most frequently used ligand for this purpose because it binds with high affinity to several types of integrins present on the surface of mammalian cells. Part of our work involved the construction of RGD modified adenovirus. Detailed results are described in Chapter 4.

\section{Stem Cell Therapy}

Stem cells exist in all multicellular organisms and share two characteristic properties. They have prolonged or unlimited self-renewal capacity and the potential to differentiate into a variety of specialized cell types. The earliest stem cells in human life are ESC cells, derived from the inner cell mass of the blastocyst and capable to differentiate into all derivatives of the three primary germ layers: ectoderm, endoderm, and mesoderm. Besides the ES cells which can only be isolated from early embryos, there are other types of stem cells in the mature tissues of all aged mammals, the adult stem 
cells. Adult stem cells have unlimited self-renewal capacity and more restricted differentiation potential. They multiply by cell division to replenish dying cells and regenerate damaged tissues. The most famous adult stem cells are hematopoietic stem cells (HSCs) which give rise to all the blood cell types and lymphoid lineages. Bone marrow also contains a population of adult stem cells named MSCs.

Although bone marrow is considered as the primary source of MSCs, they can be isolated from other tissues, including adipose tissue, trabecular bone, synovium, skeletal muscle, deciduous teeth, and human umbilical cord blood [22-27], suggesting the diverse distribution of MSCs in a body. However, MSCs derived from organs other than bone marrow exhibit limited differentiation potential [28, 29]. Among all types of stem cells, MSCs have attracted special attentions because of its wide application as regenerative medicine. ES cells were first studied as regenerative medicine because of their selfrenewal capacity and differentiation potential. However, direct injection of highlypluripotent ES cells into ectopic organ often give rise to teratoma, a benign tumor containing derivatives of all three germ layers [30]. MSCs are less potent to induce teratoma or other malignant transformation as they only have restricted differentiation potential [31]. Compared with other adult stem cells such as HSCs, mammary stem cells or neural stem cells, MSCs have a well-characterized trophic effect and immunomodulatory property, making them good candidates in treating degenerative diseases. For example, intravenous transplantation of MSCs was reported to be successful in treating systemic diseases such as graft versus host disease (GvHD) and osteogenesis imperfecta in human $[32,33]$. Wakitani et al. also reported several successful clinical cases treating cartilage defects with MSCs [34]. Nevertheless, primary MSCs have also been employed in regenerating hematocytes, tendon, bone marrow, muscle, and other connective tissues [35-39].

Bone marrow derived mesenchymal stem cells (BMSC) are morphologically defined as plastic, adherent, pluripotent fibroblast-like cells. BMSCs are stem cells because of their stem cell-like properties such as unlimited self-renewal capacity and potential for multilineage differentiation. Primary BMSCs can be expanded for 34 50 population doublings $(\mathrm{PD})$ without losing their native characteristics. BMSCs can differentiate into a variety of cell types including osteoblasts, chondrocytes, and adipocytes under in vitro and in vivo conditions [40].

\section{Progress in Stem Cell Therapy}

There are two major directions of applying BMSCs to human islet transplantation, as trophic mediators to support islet function or as immune modulators to prevent graft rejection. 


\section{BMSCs support islet function}

In vivo studies using BMSCs to treat diabetes used to support a hypothesis that BMSCs might differentiate into insulin-producing $\beta$ cells or they might induce endogenous progenitor proliferation/differentiation. Ezquer et al. demonstrated that systemic administration of BMSCs increased $\beta$ cell mass and reverted hyperglycemia in streptozotocin (STZ) induced type 1 diabetic mice [41]. However, whether BMSCs can directly replenish the loss of $\beta$ cells is still under debate. The native $\beta$ cells are derived from neural crest cells during the process of neurulation, while BMSCs are derived from mesoderm. Although the number of islets does not increase throughout human life, insulin-producing $\beta$ cells do proliferate according to several reports [41-43]. Hess et al. first raised doubt that MSCs did not directly rescue the pancreatic injuries by in vivo differentiation and migration but instead induced endogenous pancreatic tissue repair in an unknown manner [44]. Lately, both Choi et al. [45] and Dor et al. [46] confirmed that new pancreatic $\beta$-cells are derived from the expansion of pre-existing $\beta$ cells rather than exogenous stem cells through the convincing lineage tracing studies. Since then, emerging evidence suggested that BMSCs supported islet function through a predominant paracrine model, or in other words served as a "trophic mediator."

BMSCs actively produced HGF, which is a potent mitogen to many cells including pancreatic $\beta$-cells [47]. For example, in a study using human BMSCs to treat STZ induced diabetic mice, Lee et al. demonstrated that the major effect of human BMSCs treatment was to increase the number of mouse islets and mouse insulinproducing cells, which were most likely to arise from the proliferation, migration, and neural differentiation of the nearby endogenous mouse neural stem cells [42].

Most studies suggested that BMSCs actively participate in angiogenesis of human islets by secreting VEGF and HGF which are potent angiogenic factors [48]. Izumida et al. reported that proliferative activity and differentiative response in the pancreatic ductal cells were significantly raised once rats were treated with HGF positive BMSCs [49]. Sordi et al. found that BMSCs of bone marrow origin facilitated the restoration of normoglycemia and the neovascularization of the islet graft [50]. which was confirmed by a recently study by Rackham et al. that islets co-transplanted with BMSCs maintained a morphology that more closely resembled that of islets in the endogenous pancreas both in terms of size, endocrine and endothelial cell distribution [51]. We also demonstrated that islet/BMSC co-transplantation under the kidney capsule of diabetic mice formed a more intense and function vasculature [52]. Other studies suggested that BMSCs may also differentiate into endothelial cells and directly assist the neo-vessel formation [53]. Silva et al. reported the transdifferentiation of BMSCs into an endothelial phenotype in a canine ischemia model, which is supported by the report from Ito et al. that BMSCs directly differentiate into a van-willebrand-factor-positive vascular endothelial cell type to improve the islet graft morphology and function [54]. However, these results should be explained cautiously because the new-formed vessels may arise from native BMSCs instead of infused BMSCs. 


\section{BMSCs prevent graft rejection}

The allograft rejection from the host innate immune system is another major issue for islet transplantation. The most potent antigen-presenting cells, dendritic cells (DCs) play a key role in allograft recognition and rejection. Briefly, the precursor monocytes migrated through the capillaries into tissue and differentiate into immature DCs. Once encountering foreign cells, DCs process and present the allogeneic antigen through major histocompatibility complex class II (MHC II) to the T cell receptor (TCR) of native T cells (T0) and promote T cell activation into cytotoxic T cells (Tc), helper T cells (Th) and memory T cells (Tm), while immature DCs themselves undergo maturation simultaneously. Tc cells then mediate the acute immune attack to the allograft. Th cells recruit and activate more immunocytes including Tc, macrophages and B cells and lead to an enlarged immune response. Tm cells circulate in the host body and mediate the long-term rejection.

BMSCs are hypoimmunogenic cells, expressing MHC1 but not MHC II. They also lack co-stimulatory molecules including CD14, CD86, CD40L and CD95L (FasL) [55]. Therefore, BMSCs can evade the alloreactive T cells and natural killer (NK) cells in a similar manner like cancer cells $[56,57]$. Most researchers believe that MSCs induced immune tolerance by inhibiting $\mathrm{T}$ cell activation and proliferation. Two models have been proposed: 1) BMSCs inhibited T cell activation through physical contact (the direction inhibition model) and 2) BMSC produced immunosuppressive soluble factors to reverse the maturation of antigen-presenting DCs and subsequently led to T cell inactivation (the indirect inhibition model) (Figure 1-2).

The direct inhibition model proposed that the rate of $\mathrm{T}$ cell inhibition was increased when cell contact between BMSCs and T cells was allowed [58]. Krampera et al. showed that the inhibitory activity of BMSCs was abrogated when BMSCs were cocultured with T cells in a transwell system or when BMSCs were replaced by BMSC culture supernatant [59]. Ding et al. supported the direct inhibition model by reporting that the extracellular matrix proteins such as matrix metalloproteinase (MMP)-2 and MMP-9 of BMSCs cleaved the IL-2 receptor from the surface of T cells and suppress T cell responses [60]. However, overwhelming studies supported the indirect model, showing BMSCs actively secreted multiple soluble immunosuppressive factors such as indoleamine-pyrrole 2, 3-dioxygenase (IDO), HGF, transforming growth factor- $\beta$ (TGF$\beta$ ), prostaglandin E2 (PGE2) and inhibited the alloreactivity of T cells in recipients and prolonged the allograft survival [58, 61-64].

However, it is worth noting that multiple discrepancies exist among these soluble factors secreted by MSCs. For example, evidences from Beyth et al. showed that adding neutralizing antibody to human TGF- $\beta$ had no effect on the inhibitory activity of MSCs[65], in contrast to the report by Di Nicola et al.[58]. Ryan et al. reported constitutive expression of IL-10 by MSCs while Beyth et al. only detected IL-10 in MLR/MSCs co-culture [55, 65]. Moreover, studies by Tse et al. showed that none of IL10 , TGF- $\beta$ or PGE2 produced by MSCs was responsible for the T-cell inhibitory effect [66]. Although such discrepancies could be explained by the multiple sources and 


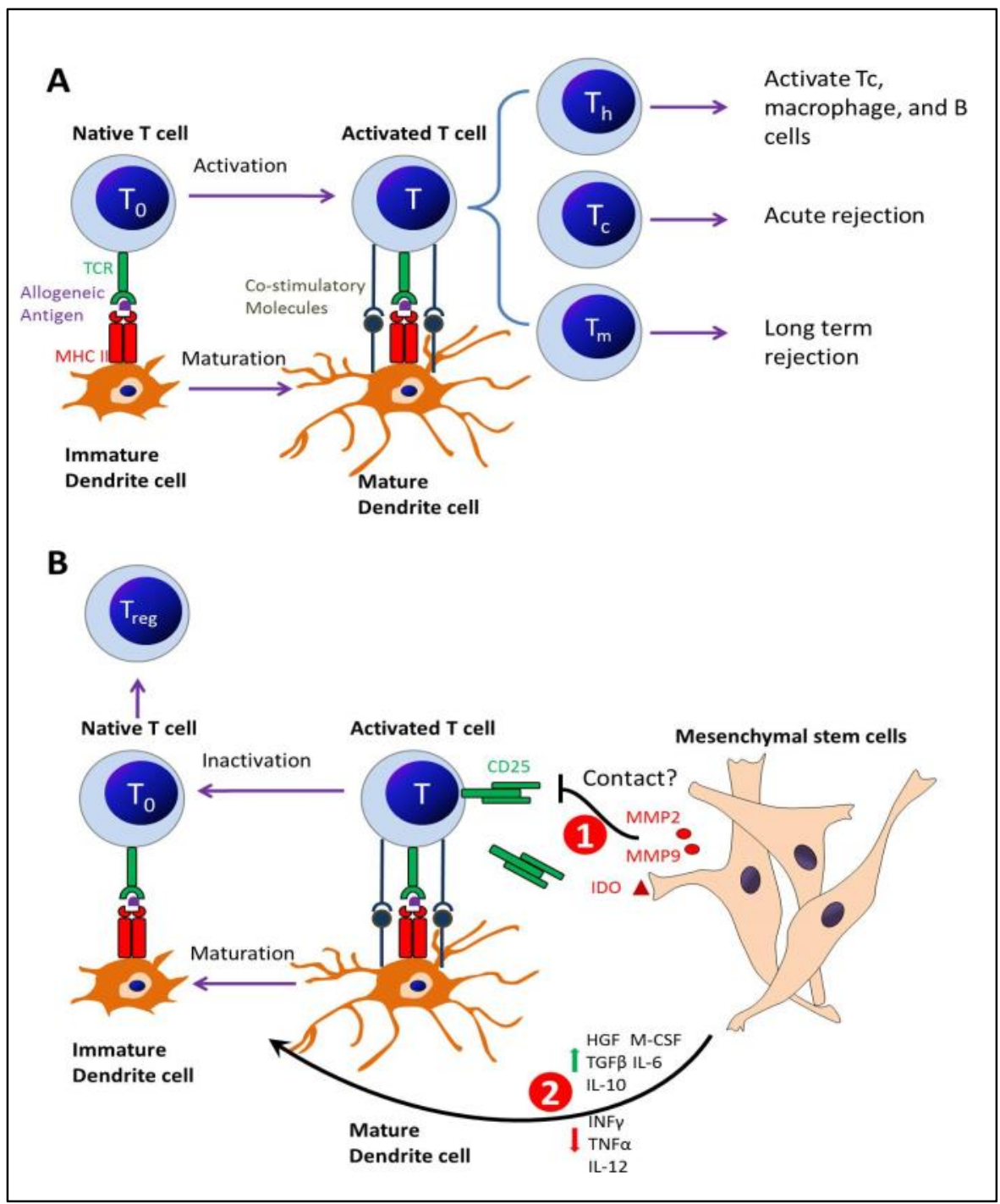

Figure 1-2. MSCs inhibit $T$ cell activation

A. The allograft rejection mediated by T cells. Briefly, antigen-presenting Dendrite Cells (DCs) present allogeneic antigen through MHC II to the TCR of T0 cells. With assist from co-stimulatory molecules DCs become activated into mature DCs and simultaneously promote $\mathrm{T}$ cell activation into $\mathrm{Tc}, \mathrm{Th}$ and $\mathrm{Tm}$. Then the activated $\mathrm{T}$ cells lead to a cascade of immune responses to mediate acute graft rejection and long term graft rejection. B. MSCs inhibit T cell activation through two mechanisms. (1) Direct T inhibition, which is achieved by a so-far unknown cell-cell contract mechanism or MMP2/MMP9 mediated CD25 cleavage on the surface of activated T cells. (2) Indirect T cell inhibition, which is achieved by releasing soluble factors to inhibit and reverse DCs maturation. The antigen presenting process through immature DCs which in lack of costimulatory molecules leads to inactivation of activated $\mathrm{T}$ cells, proliferation of immunosuppressive Treg and allogeneic tolerance. 
lineages of MSCs and variation in culture conditions among the research groups, the underlying signal transduction pathway between soluble factors released by BMSCs and the antigen-presenting DC require further exploration. Moreover, most of these studies are conducted using an in vitro cell culture instead of animal model. One goal of our work is to resolve the mysteries of the immunomodulatory effect of BMSCs.

The immunomodulatory effect of BMSC makes them good candidate in organ transplantation. Ding et al. reported that BMSCs co-transplanted under the kidney capsule of immune competent mice protected islet grafts by inhibiting the alloreactivity of infiltrating T cells [60]. Longoni et al. reported that BMSCs induced a reduction of inflammatory cytokines and improved the viability of islets infused into the portal vein of diabetic rats [67]. Li et al. reported reduced $\mathrm{Th} 1 / \mathrm{Th} 2$ ratio, Tc cells and $\mathrm{Tm}$ cells number and suppressed DCs maturation once BMSCs were co-transplanted with allograft islets under the kidney capsules of diabetic C57LB/6 mouse [68]. Kim et al. reported the combined use of autologous BMSCs and low-dose cyclosporine A to further prolong graft survival after allogeneic rat islet transplantation [69]. BMSCs were also used to prevent the allograft rejection in the transplantation studies of other organs including kidney,[61, 70] skin[71] and heart [62, 72, 73] (Table 1-4).

\section{Stem Cell Based Gene Therapy}

In the last decade, gene therapy and stem cell therapy are frequently combined together to enhance the therapeutic effect, unusually referred as stem cell based gene therapy. Stem cell based gene therapy can be applied on human islet transplantation from two directions: 1) Stem cells were used as gene delivery vehicles to express therapeutic gene in the target sites and 2) stem cells were reprogrammed or transdifferentiated by genetic modification to replenish the defect cells or tissues (regenerative medicine).

Traditional gene therapy requires direct transduction on targeted tissue so that transgene can be introduced and expressed afterward. The gene transfer process normally takes 16 24 hours and has several drawbacks in human islet transplantation. The first is the loss of islet viability and function during the transduction process. One extra day of in vitro culture can make a big difference in the function, viability and quantity of islets needed for optimal transplantation outcome. The second issue is the toxicity and immunogenicity of adenoviral vectors. Adenoviral at high MOI will kill islets. Even at a moderate MOI which is not toxic to human islets, adenovirus will increase the immunogenicity of human islets and consequently induce strong immune rejection. Therefore for some soluble factors whose effect can be exerted from outside of the cytoplasm, use stem cells as nursing cells to deliver therapeutic genes can be a smart strategy to overcome the issues with direction transduction (Figure 1-3). Part of our work focused on the feasibility of using BMSC as a gene delivery vehicle to support the survival of human islets after transplantation and the detailed results are presented in Chapter 5.

BMSCs not only regenerate mesenchymal tissues such as chondrocytes, 
Table 1-4. BMSCs improve solid organ transplantation

\begin{tabular}{|c|c|c|}
\hline Organs & Experimental design & Therapeutic effects of BMSCs \\
\hline \multirow[t]{3}{*}{ Kidney } & $\begin{array}{l}\text { Human of } 14 \text { subjects. In vitro MLR study of donor } \\
\text { BMSCs against recipients lymphocytes. }\end{array}$ & $\begin{array}{l}\text { BMSCs inhibit the proliferation of Th and Tc from the } \\
\text { recipients by cell-cell contact and IL-10 and IDO. No effect } \\
\text { on B or NK cells [61]. }\end{array}$ \\
\hline & Human of 2 subjects. Autologous BMSCs were injected & BMSCs inhibit the proliferation of Tm and Tc. Increase \\
\hline & IV into recipients at day 7 posttransplantation. & Treg percentage [70]. \\
\hline Skin & $\begin{array}{l}\text { Baboons study. Donor BMSCs from MHC mismatched } \\
\text { donor were injected IV into recipients on the day of } \\
\text { transplantation. }\end{array}$ & $\begin{array}{l}\text { BMSCs inhibit lymphocyte reactivity and prolong the graft } \\
\text { survival and suppress the proliferation. IL-2 partially } \\
\text { reverse the effects of BMSCs [71]. }\end{array}$ \\
\hline \multirow[t]{3}{*}{ Heart } & $\begin{array}{l}\text { Rat. In vitro expanded BMSCs were injected IV } 1 \text { week } \\
\text { before and on the day of transplantation. }\end{array}$ & $\begin{array}{l}\text { BMSCs reduce the alloreactivity of recipient's T cells and } \\
\text { shift the Th1/Th2 balance to immunosuppressive Th2 [72]. }\end{array}$ \\
\hline & $\begin{array}{l}\text { Mice. In vitro expanded BMSCs were injected IV before } \\
\text { transplantation. }\end{array}$ & $\begin{array}{l}\text { BMSCs induce donor-specific Treg proliferation and } \\
\text { impaired Th1 alloreactivity. Donor-specific Treg do not } \\
\text { lead to immune tolerance of allograft from third-party [73]. }\end{array}$ \\
\hline & $\begin{array}{l}\text { Rat. BMSCs were injected IV with a short course of low- } \\
\text { dose mycophenolate. }\end{array}$ & $\begin{array}{l}\text { BMSCs induce tolerance by secretion IDO and interaction } \\
\text { with DCs [62]. }\end{array}$ \\
\hline \multirow[t]{4}{*}{ Islet } & Rat. In vitro expanded BMSCs co-infused into liver. & $\begin{array}{l}\text { Reduce islet number needed for reversal of diabetes } \\
\text { promote revascularization [54]. }\end{array}$ \\
\hline & $\begin{array}{l}\text { Rat. In vitro expanded BMSCs were co-transplanted into } \\
\text { omental pouch. }\end{array}$ & $\begin{array}{l}\text { Inhibit Th1 cell activation promote IL-10 producing CD4+ } \\
\text { T cells [74]. }\end{array}$ \\
\hline & $\begin{array}{l}\text { Mice. In vitro expanded BMSCs were co-transplanted } \\
\text { beneath the kidney capsule }\end{array}$ & $\begin{array}{l}\text { BMSCs secreted MMP } 2 \text { and MMP9 to cleave CD } 25 \text { from } \\
\text { IL-1R and thus lead to interleukin-2 hyporesponsiveness in } \\
\text { T-cells [60]. }\end{array}$ \\
\hline & $\begin{array}{l}\text { Monkey. BMSCs from donor and third party were co- } \\
\text { infused into portal vein and injected IV thrice at day } 4,5 \\
11 \text { after transplantation. }\end{array}$ & $\begin{array}{l}\text { BMSCs prolong graft viability and function, probably by } \\
\text { increasing Treg proliferation in peripheral blood [75]. }\end{array}$ \\
\hline
\end{tabular}




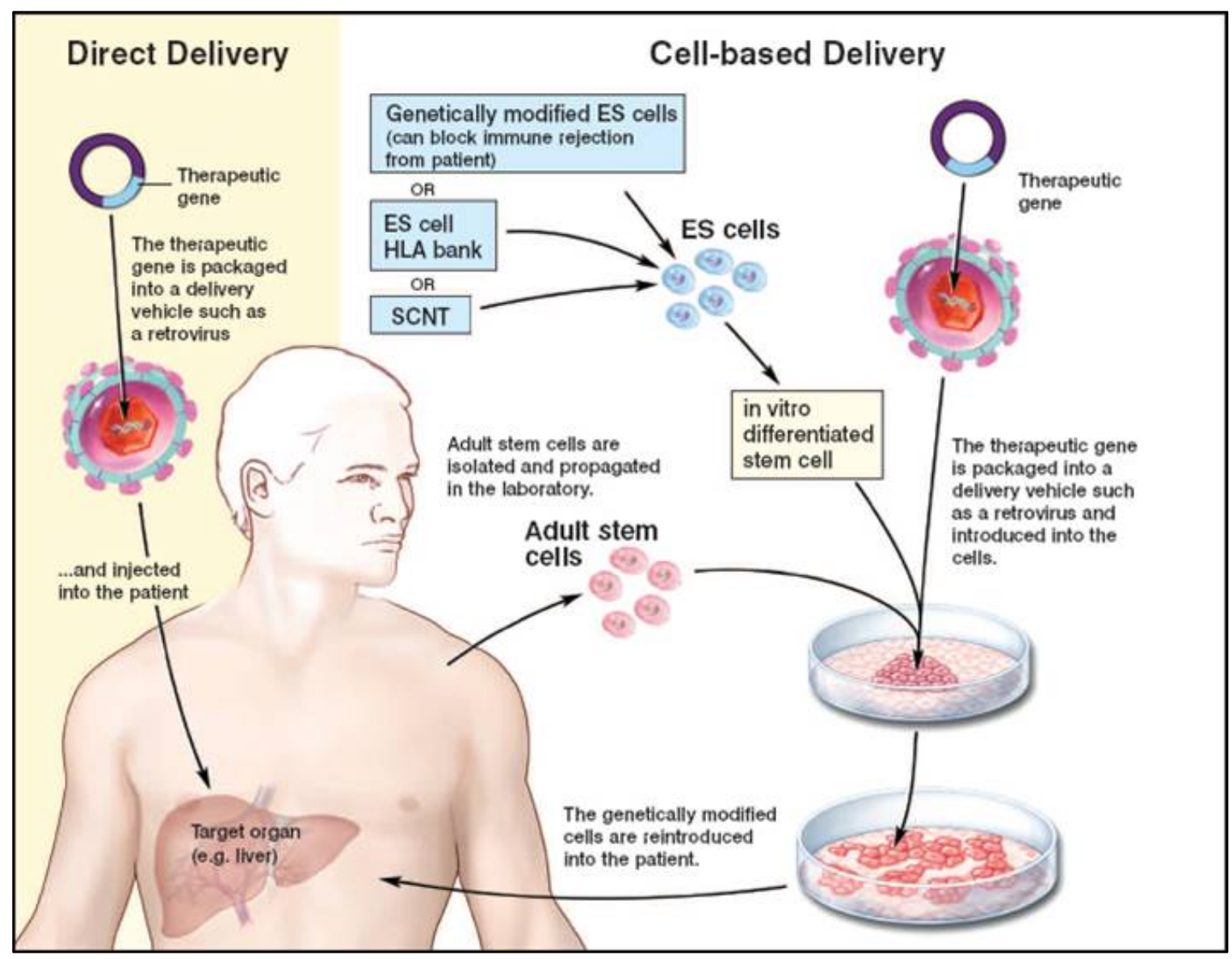

Figure 1-3. Gene therapy and stem cell based gene therapy

In vivo gene transfer involves direct administration of the vector in the tissue of interest. Ex vivo gene transfer requires collection of cellular targets from the patient. The cells are treated in culture with the vector. Cells expressing the therapeutic transgene are harvested and given back to the patient. 
osteoblasts, and adipocytes but also undergo transdifferentiation into cells from other lineages upon proper induction, indicating plasticity of these adult stem cells. Gene transfer into primary BMSCs may greatly promote their transdifferentiation into insulinproducing cells even islet-like cluster, which may replenish the current shortage in the supply of donor islets. Chen et al. first reported in vitro transdifferentiation of rat MSCs into functional insulin-producing islet-like cells that actively controlled blood glucose level in diabetic rats [76]. Then Karnieli et al. and Li et al. independently reported the generation of insulin-producing cells from human MSCs which were genetically manipulated to overexpress $\mathrm{Pdx}-1$ with retrovirus and adenovirus, respectively $[77,78]$. The research in this area provided new insights and has bright future in human islet transplantation. However, we just initiate our work in this area and it is out of the scope of this thesis.

\section{Diabetic Humanized Animal Model}

Immune deficient animal models are most frequently used for the research in human islet transplantation. These models provide optimal environment for the engraftment of islets from multiple sources because of the absence of immune rejection. However, these models can only be used to study the function of islets against the nonimmune challenges such as hypoxia, inflammatory cytokines and early graft apoptosis, which is the PNF of islets.

To understand the physiology of islet grafts under the immune rejection of the recipient, a humanized mouse model was introduced. Lenny Schultz first introduced the idea of humanized mouse in which human immune system was rebuilt in mouse by injecting and settling human peripheral blood mononuclear cells (PBMCs) $[79,80]$. The success in building human immunity in mice relies on the species and strain of mouse. For example, human immunity can be readily built by injecting PBMCs into NOD.CgPrkdc $^{\text {scid }}$ IL2 rg $^{\text {tm } 1 \mathrm{Wjl}} / \mathrm{SzJ}$ (NSG) mice but not Nonobese diabetic severe combined immune deficient (NOD-SCID) mice. This may be a result of the knock-out of IL-2rgamma, which has great impact on the settlement of PBMCs, in the NSG mice. Another mouse model was NOD.Cg-Rag $1^{\text {tm1Mom }}$ Ins $2^{\text {Akita }} \mathrm{Il} 2 \mathrm{rg}^{\mathrm{tm} 1 \mathrm{Wjl}} / \mathrm{SzJ}$ (NRG-Akita) mice, which spontaneously develop diabetes from 5-7 weeks. However, the difficulty of using NRG mice is that not all mice develop similar extent of diabetes at a same pace. To control the extent of diabetes, we choose STZ induced NSG mice for islet transplantation studies.

The risk of GvHD is another factor need to be taken into consideration. The mature PBMCs has the potential to recognize and attack the xenogeneic organs after infusion. GvHD is usually characterized by the weight loss, hunched posture, ruffled fur, reduced mobility, extremely low blood glucose and tachypnea and will lead to animal death in one day or two. To avoid GvHD, Dose of PBMCs used for infusion is important. The most frequently reported dose is $5 \times 10^{6} \mathrm{PBMCs} /$ mouse, which is enough to build human immunity but less potent to induce GvHD. However, even at such a low dose, GvHD is only pended but not avoided. GvHD will occur at 5 10 weeks after infusion in most cases, therefore we only buy ourselves a limited window to evaluate the immune 
rejection of human islets even at the safety dose of PBMCs. Recently, a lot of efforts have been put onto the maturation of human immunity in mice using hematopoietic stem cells. It was recently demonstrated that the immunocytes matured inside the body of mouse has less potential to attack organs of mouse and therefore less likely to induce GvHD like symptoms.

Induce diabetes in humanized mice or vice versa (humanize diabetic mice) are both tricky task. We chose the latter strategies because of two reasons: 1) it provides direct contrast of the responses of animals to transplanted human islets in the absence and presence of human islets and 2) the chemical used to induce diabetes, STZ is strong immune activation drug and the injection of STZ into humanized mouse will elicit a fast and strong GvHD and cause animal death in less than 2 weeks. NRG-Akita mouse was not used in this study purely due to cost considerations. Because mice develop diabetes at different pace but islets always come together and vary from preparation to preparation. We need a strategy which is capable of develop suitable extent of diabetes in most mice in the same day so all of them are suitable for human islet transplantation.

To generate diabetic humanized mouse, we first induce diabetic in NSG mice by intraperitoneally injection of STZ. We wait $3 \sim 4$ week until all mice showed similar extent of diabetes (blood glucose $300 \sim 500 \mathrm{mg} / \mathrm{dL}$ ). Then islets were transplanted with or without BMSCs. After additional 4 weeks, PBMCs were injected to introduce human immunity. Detail experiments are described in Chapter 6. 


\section{CHAPTER 2. SPECIFIC AIMS}

\section{Aim I. Adenovirus Mediated XIAP Expression to Prevent the Early Graft Apoptosis in Human Islet Transplantation}

The main objective of this part of research is to find the best anti-apoptotic gene to prevent the early apoptosis in human islet transplantation. Our formal lab members identified several target genes in the apoptotic pathway of human islet. For example, Feng Li silenced iNOS gene while Guofeng Cheng silenced caspase 3 using RNA interference for in vitro protection of human islets from inflammatory cytokines [5, 6]. Ravikiran Panakanti blocked IL-1 pathway by overexpressing IL-1 receptor antagonist (IL-1Ra) to prevent the early graft loss after islet transplantation into NOD-SCID mice $[4,8]$. All these previous work inspired our research and hereby we hypothesized that XIAP over expression can be a more effective strategy to prevent the early graft apoptosis in human islet transplantation. We propose several objectives in this part of research and discuss in detail in Chapter 3.

1) Learn the techniques in the culture of rat insulinoma cells (INS-1E) and human islets.

2) Learn the techniques in the generation, amplification, tittering and storage of adenovirus.

3) Test the transgene expression of XIAP in genetically modified human islets.

4) Test whether XIAP expression improves the outcome of human islet transplantation in immunodeficient NOD-SCID mice.

\section{Aim II. RGD Peptide Modified Adenovirus Encoding XIAP and HGF to Simultaneously Prevent Early Graft Apoptosis and Promote Revascularization to Improve the Outcome of Human Islet Transplantation}

The main objective of this research is to improve the transduction efficiency of traditional adenoviral vectors. In 2004, when we first initiated gene therapy project, we used plasmid and lipid-mediated transfection to introduce gene of interest into human islets [12]. However, the gene transfer efficiency of this strategy was extremely low. In 2006 we converted the plasmids to adenoviral vectors, which significantly improved the gene transfer efficiency [7]. However, the titer of adenovirus must be strictly controlled because high titer of virus is known to induce toxicity in human islets. After that we designed several bipartite adenoviral vectors so that two therapeutic genes can be coexpressed simultaneously without increase the viral titer $[4,8]$. But this strategy was incompetent to reduce the viral titer for optimal transgene expression. Hereby we were trying to modify the surface of adenovirus so that less viral particles can be used to transduce human islets without compromising the transduction efficiency. We propose several objectives in this part of research and discuss in detail in Chapter 4.

1) Learn the techniques in the induction of moderate diabetes in mice. 
2) Compare the transduction efficiency of unmodified adenovirus and RGD modified adenovirus.

3) Test the transgene expression of XIAP and HGF in genetically modified human islets.

4) Test whether genetically modified human islets improve the outcome of human islet transplantation in immunodeficient NOD-SCID mice.

\section{Aim III. Use BMSCs as Gene Delivery Vehicles to Express HGF and IL-1Ra to Improve the Outcome of Human Islet Transplantation}

The main objective of this study is to determine whether gene therapy can be achieved without the direct gene transfer into human islet. We successfully decreased the viral titer for optimal transgene expression in the previous study. However, the use of virus, at whatever titer, will raise safety concerns such as immunogenicity and tumorigenicity. Moreover, before islet transplantation can be performed, gene transfer into human islets will cost one extra day, which makes great difference in the viability and function of islets and thus the outcome of islet transplantation. We hypothesized that BMSC can be genetically modified by adenovirus before the arrival of islet preparations and co-transplanted with fresh human islets to treat type 1 diabetes. We propose several objectives in this part of research and discuss in detail in Chapter 5.

1) Test the transduction efficiency of adenovirus on BMSCs.

i. Test whether the adenoviral transduction affects the gene expression prolife and stem cell like properties of BMSC.

ii. Test the duration and level of transgene expression in genetically modified BMSCs.

2) Test the protection of genetically modified BMSCs to human islets in islet perifusion study in vitro.

3) Test whether islet/BMSCs cotransplantation improve the outcome of human islet transplantation in immunodeficient NOD-SCID mice.

\section{Aim IV. Use BMSCs to Prevent the Immune Rejection of Islet Allografts in Humanized NSG Mice}

Gene therapy is incompetent to prevent the immune rejection, while stem cells can be an effective strategy to overcome the immune rejection. In this study, we borrowed an up-to-date humanized animal model to determine immunomodulatory effect of BMSC to protect human islets in the presence of human immunity. We propose several objectives in this part of research and discuss in detail in Chapter 6.

1) Build human immunity in STZ-induced diabetic NSG mice through intraperitoneal injection of human PBMCs.

2) Determine the immunomodulatory effects of BMSCs in vitro.

i. Characterize the surface markers of BMSCs. 
ii. Test whether BMSCs can suppress the proliferation and activation of PBMCs in the mixed lymphocyte reaction (MLR).

iii. Test whether BMSCs can promote the regulatory T cells in MLR.

3) Determine the mechanism underlying the immunosuppressive effect of BMSCs.

i. Determine whether BMSC alone can suppress the proliferation and activation of PBMCs.

ii. Determine the role of IL-10 in the immune modulation mediated by BMSCs.

iii. Determine the sources of IL-10 production in the MLR/BMSC mixture.

4) Test the tropic effect of BMSCs in vitro.

i. Test the gene expression profile of BMSC.

ii. Test whether BMSC can improve the insulin production of islets under the challenges by inflammatory cytokines.

5) Test whether BMSCs prevent the graft rejection in human islet transplantation in a humanized immune-competent mice model.

i. Test whether BMSCs prevent T cell infiltration into the transplantation site.

ii. Test whether BMSCs increase Tregs.

iii. Test whether BMSCs improve early engraftment and revascularization of human islet transplantation.

\section{Aim V. Use Small Molecular Compound Drug to Prevent the Immune Rejection of Islet Allografts in Humanized NSG Mice}

BMSCs were capable to prevent the immune rejection of human islets in humanized mouse model. However, BMSCs will be sequestered in the lung after intravenous injection, making them unsuitable for repeated dosing. Despite the hypoimmunogenic nature, BMSCs from a third party, if used in large quantity, will raise safety concerns about the immunogenicity and tumorigenicity. In this study, we synthesized a compound drug (E)-2,3,5-trihydroxy-5-(propylcarbamoyl)cyclohexyl 6-(4ethoxy-6-methoxy-7-methyl-3-oxo-1,3-dihydroisobenzofuran-5-yl)-4-methylhex-4enoate (JP-3-110) by conjugating anti-apoptotic compound 1,3,4,5-tetrahydroxy-Npropylcyclohexanecarboxamide (KZ41) and immunosuppressive compound MPA. We hypothesized that this new compound can exert the immunosuppressive effect through intravenous injection while avoiding the toxicity associated with MPA. We propose several objectives in this part of research and discuss in detail in Chapter 7.

1) Synthesis and characterization of a compound drug JP-3-110 which is composed of MPA, an immunosuppressive drug and KZ-41, a novel antiapoptotic compound for human islet transplantation.

2) Compare the anti-apoptotic effect of JP-3-110 and KZ41 against the inflammatory cytokines.

3) Compare the immunosuppressive effect of JP-3-110 and MPA on PBMCs. 


\section{CHAPTER 3. XIAP OVEREXPRESSON MEDIATED BY ADENOVIRUS TO PREVENT APOPTOSIS OF ISLET TRANSPLANTATION*}

\section{Introduction}

The loss of function of transplanted human islets is usually accompanied with the apoptosis of insulin-producing $\beta$ cells. The apoptosis of $\beta$ cells can be caused by many reasons including lack of supplies of oxygen and nutrition, poor revascularization, inflammatory cytokines, and reactive oxidative species. In most cases, islet loss occurs in the first two weeks after transplantation, and will decrease significantly due to successful revascularization thereafter $[81,82]$. Therefore, expression of an antiapoptotic gene in $\beta$ cells timely may be an effective strategy to improve islet viability post transplantation [17]. We previously reported ex vivo transduction of islets with adenoviral vector encoding human interleukin-1 receptor antagonist (IL-1Ra) to prevent IL-1 $\beta$ induced apoptotic cell death of islets [3]. We also demonstrated that the inhibition of caspase-3 pathway helped to protect human islets from apoptosis caused by inflammatory cytokines in vitro [6]. However, the apoptosis of islets usually involved multiple inflammatory cytokines and a network of signal transduction pathways (Figure 3-1). The therapeutic effect gained from the inhibition in a single factor or pathway was limited and not capable to reverse the apoptosis of islets in a whole picture. For example, IL-1Ra expression was not able to address the apoptosis of islets caused by hypoxia and ROS. A target gene with more ubiquitous anti-apoptotic effect was needed.

In this study, we identified XIAP as a target gene. XIAP is a well characterized inhibitor of apoptosis by inhibiting three types of caspase at the same time (Figure 3-1). The BIR2 domain of XIAP inhibits caspase 3 and caspase 7, while BIR3 domain inhibits caspase 9 [83-85]. Besides, XIAP is also capable of address the apoptosis from death receptor pathway and mitochondria pathway. Therefore, XIAP holds great potential to inhibit the apoptosis of human islets caused by hypoxic environment, ROS and inflammatory cytokines together.

XIAP overexpression minimizes the injury in pancreatic $\beta$-cells caused by hypoxia and reperfusion [86]. Hui and colleagues demonstrated the reversal of the negative effects of immunosuppressive drugs by XIAP overexpression on human islets [87]. XIAP has also been proven to improve the murine islet viability after isolation [88]. However, the mechanism underlying the protective effect of XIAP was also not thoroughly understood and promising long term data normoglycemic control in animal experiments is still needed. Therefore, in this study, we transduced INS-1E cells and human islets with replication deficient adenovirus encoding XIAP to determine whether XIAP overexpression protects $\beta$ cells and islets from cytokine induced cell death.

\footnotetext{
* Reprinted with permission from American Chemical Society. Wu, H, Panakanti, R, Li, $\mathrm{F}$, and Mahato, RI (2010). XIAP gene expression protects beta-cells and human islets from apoptotic cell death. Molecular Pharmaceutics 7: 1655-1666.
} 


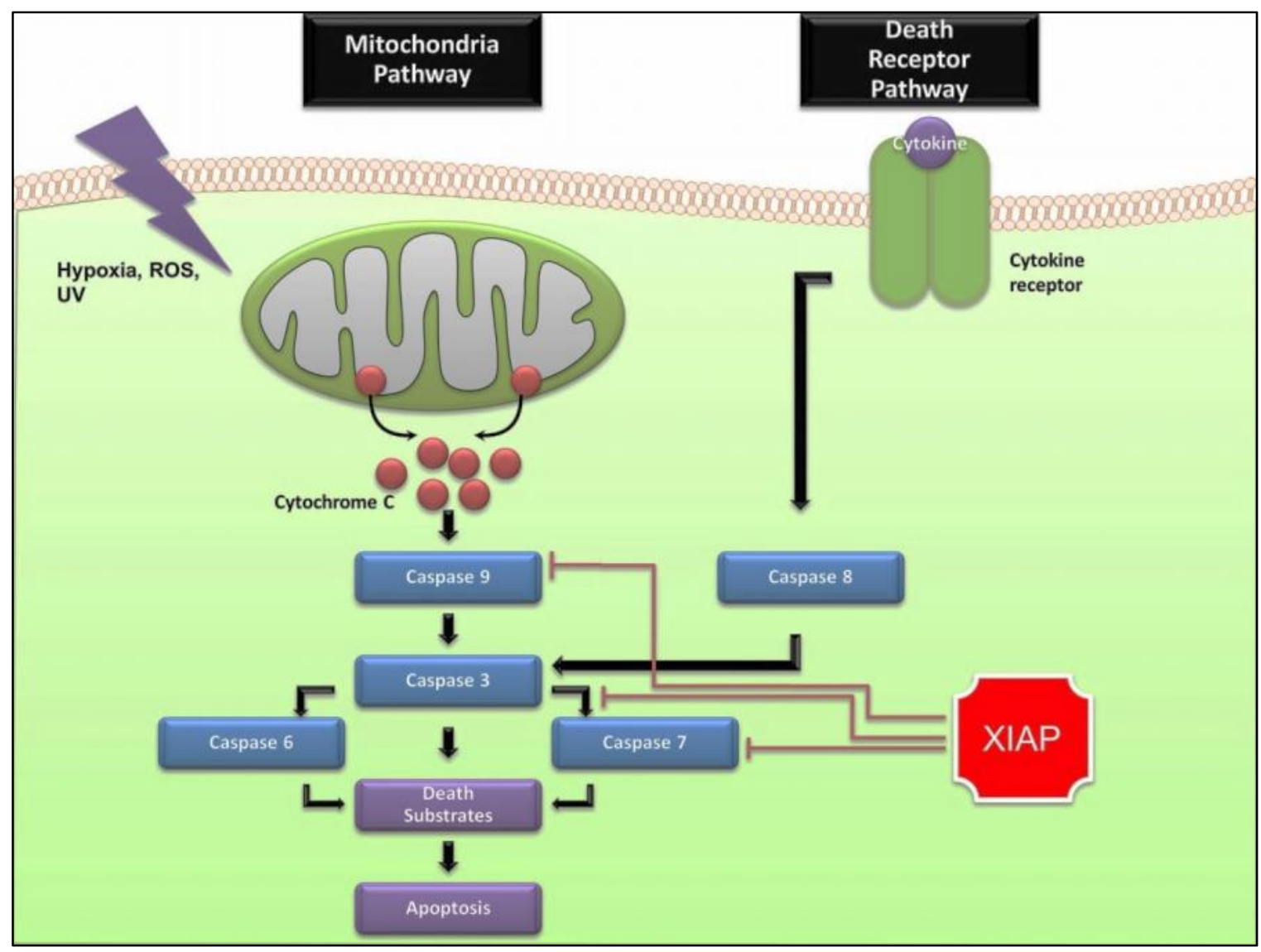

Figure 3-1. Schematic diagram showing the role of XIAP in the apoptotic pathway of human islets

XIAP inhibits caspase 3, caspase 7 and caspase 9 simultaneously. XIAP also inhibits the apoptosis caused by the mitochondria pathway (hypoxia, reactive oxygen species (ROS), and ultraviolet radiation) and death receptor pathway (inflammatory cytokines) at the same time. Therefore, we hypothesized that the anti-apoptotic effect of XIAP overexpression should be better than the gene silencing of caspase 3 or iNOS and the blocking of IL-1R. 


\section{Materials and Methods}

\section{Materials}

The replication deficient $(\Delta \mathrm{E} 1 / \Delta \mathrm{E} 3)$ adenoviral vector encoding human XIAP (Adv-hXIAP) was purchased from Vector Biolabs (Philadelphia, PA). RPMI 1640 medium was purchased from Invitrogen (Carlsbad, CA). CMRL-1066 medium was purchased from Sigma Aldrich (St. Louis, MO). Fetal bovine serum (FBS) was purchased from MediaTech Cellgros (Herndon, VA). Phosphate buffer solution (PBS) was purchased from GIBCO-BRL (Gaithersburg, MD). Human XIAP enzyme-linked immunosorbent assay (ELISA) kits and cytokines such as IL-1 $\beta$, TNF- $\alpha$, and interferon (IFN) $-\gamma$ were purchased from R\&D Systems (Minneapolis, MN). Human Insulin ELISA kits were purchased from Alpco Diagnostics (Windham, NH). RNA extraction kit and Caspase-Glo 3/7 assay kit, DeadEnd Colorimetric terminal deoxynucleotidyl transferase dUTP nick end labeling (TUNEL) system, and APO-DIRECT TUNEL system were purchased from Promega (Madison, WI). SYBR Green real-time PCR master mix and reverse transcription reagents were purchases from Applied Biosystems (Foster city, CA).

\section{Virus amplification and titer determination}

Adv-hXIAP was transduced into 293 cells. At $48 \mathrm{~h}$ post transduction, almost $90 \%$ of the cells were detached from the flask. Then, the cells were collected, centrifuged and freezed and thaw three times to release Adv-hXIAP viral particles. Then, the viral solution was used to determine the viral titer using an Adeno-X rapid titer kit from Clontech (Mountain View, CA). Briefly, different dilutions of Adv-hXIAP was taken and transduced to 293 cells, using untransduced cells as a control. After $48 \mathrm{~h}$ posttransduction, 293 cells were fixed and incubated with Anti-Hexon antibody, followed by a secondary horse radish peroxidase (HRP)-conjugated antibody. Then the cells were stained with 3, 3' diaminobenzidine tetrahydrochloride (DAB) substrate and positively stained cells were counted under a microscope, and the titer was determined.

\section{Cell and islet culture and transduction}

INS-1E cells, a gift from Professor Claes B. Wolheim (University Medical Center, Geneva, Switzerland) were seeded at a density of 106 cells per 24-well plate. After 24h, INS-1E cells were transduced with Adv-hXIAP of indicated MOI for $3 \mathrm{~h}$ followed by washing and further culturing for 2 days. Human islets were received from one of the several Islet Cell Resource (ICR) Centers through ICR services for Basic Science Applications, in culture media at $4^{\circ} \mathrm{C}$. On receiving, the islets were cultured in CMRL-1066 medium containing $10 \%$ FBS and $1 \%$ Penicillin Streptomycin. INS-1E cells and human islets were transduced with Adv-hXIAP, while nontransduced human islets as well as the islets transduced with Adv-LacZ were used as controls. To determine the optimal MOI of Adv vectors in terms of plaque forming units (pfu) per IE, $1000 \mathrm{IE}$ 
were incubated with diluted Adv-hXIAP in $300 \mu l$ media in a 96 well plate for $12 \mathrm{~h}$, followed by washing and further culturing in $1 \mathrm{ml}$ of media in 24-well plates for 2 days.

\section{Quantitative real-time PCR}

INS-1E cells were collected to determine XIAP mRNA level by quantitative Real-time PCR post-transduction. Briefly, INS-1E cells were collected and total RNA was isolated using RNAeasy mini isolation kit from Promega (Madison, WI) 3 days posttransduction. RNA concentration was measured by UV spectrophotometer. Then $170 \mathrm{ng}$ of extracted RNA was converted into cDNA using MultiScirbe reverse transcriptase and random hexamers (Applied Biosystems, Inc., Foster City, CA) by incubation at $25^{\circ} \mathrm{C}$ for $10 \mathrm{~min}$, followed by reverse transcription at $48^{\circ} \mathrm{C}$ for $30 \mathrm{~min}$ and enzyme inactivation at $95^{\circ} \mathrm{C}$ for $5 \mathrm{~min}$. Then $2 \mu \mathrm{l}$ cDNA was used as a template and analyzed by SYBR Green-I dye universal PCR master mix on a LightCycler 480 Instrument. Caspase 3 mRNA expression was also determined by the same method. $\beta$-actin was used as an internal control. All samples were run in triplicates. The primer sequences were as follows: human XIAP (forward) 5'-TGT TTC AGC ATC AAC ACT GGC ACG-3', (reverse) 5'TGC ATG ACA ACT AAA GCA CCG GAC-3'; rat caspase 3 (forward) 5'-CAT GAC CCG TCC CTT GAA-3', (reverse) 5'-CCG ACT TCC TGT ATG CTT ACT CTA-3'; rat $\beta$ actin (forward) 5'-AGT CAT GTA CGT AGC CAT-3', (reverse) 5'-CTC TCA GCT GTG GTG GTG-3'.

\section{ELISA and western blot}

At the indicated time post-transduction, INS-1E cells or human islets were collected to determine XIAP protein level by ELISA or Western Blot. Total protein was extracted by lysing INS-1E cells or human islets with RIPA buffer (Sigma) and stored in -80 oC for ELISA and Western Blot study. For ELISA, XIAP levels were measured as per the manufacturer's protocol (R\&D Systems, Minneapolis, MN). For Western Blot analysis, the samples were mixed with $6 \times$ Laemmli sodium dodecyl sulfate (SDS) buffer (Boston BioProducts) and then boiled for $5 \mathrm{~min}$ to denature the protein. Then groups of samples were loaded to 4\%-15\% Tris- $\mathrm{HCl}$ Precast Polyacrylamide Gel (Bio-Rad, Hercules, CA) for electrophoresis and subsequently transferred to Immobilon Polyvinylidene Fluoride (PVDF) membrane (Millipore). After blocking with 3\% bovine serum albumin in $1 \times$ PBST (PBS containing $0.05 \%$ Tween-20) for $1 \mathrm{~h}$ at room temperature, the membranes were further incubated with goat polyclonal IgG to XIAP and actin (Santa Cruz) primary antibodies (1:500) overnight at $4{ }^{\circ} \mathrm{C}$. Membrane was then incubated with HRP-conjugated rabbit polyclonal antibody to goat (1:10000) (Abcam) for $1 \mathrm{~h}$ at room temperature. Target proteins were detected by enhanced chemiluminescence (ECL) detection kit (GE Healthcare Life Sciences, Pittsburgh, PA). Total protein concentration of INS-1E cells and islet extracts was also determined using the bicinchoninic acid (BCA) protein assay kit to normalize the results. Actin was used as an internal control for Western Blot. 


\section{Caspase detection}

Caspase-Glo 3/7, 8 and 9 assay kits were used to analyze caspase 3/7, 8 and 9 activities, respectively, as per the manufacturer's protocol (Promega). This assay kit provides a proilluminescent caspase substrate, DEVD, when cleaved by caspases, will release luciferin to quantitatively determine caspase concentration. Briefly, $48 \mathrm{~h}$ following transduction and cytokine cocktail treatment for additional 3h, $100 \mu \mathrm{l}$ Caspase-Glo reagent was added to $100 \mu \mathrm{l}$ of culture supernatants in 96-well plates and incubated at room temperature for $1 \mathrm{~h}$. The contents were then transferred into culture tubes and luminescence was determined using a luminometer (Berthold, Germany).

\section{Cell viability}

Cell viability was determined using MTT assay. Briefly, following transduction with adenoviral vectors at $\mathrm{MOI}=2, \mathrm{INS}-1 \mathrm{E}$ cells were further incubated with cytokine cocktail, including $5 \mathrm{ng} / \mathrm{ml} \mathrm{IL-1} \beta, 25 \mathrm{ng} / \mathrm{ml} \mathrm{TNF} \alpha$, and $25 \mathrm{ng} / \mathrm{ml} \mathrm{IFN} \gamma$, for indicated time. At the end of the cytokine treatment, INS-1E cells were further cultured with medium containing $0.5 \mathrm{mg} / \mathrm{ml} 3$-(4,5-dimethyl-thiazol-2-yl)-2,5-diphenyl tetrazolium bromide (MTT, Sigma) for $2 \mathrm{~h}$ in $37 \mathrm{oC}$. Then the medium was removed and 200ul DMSO was added into each well to dissolve the formazan crystals. The absorbance was measured in $550 \mathrm{~nm}$ using a microplate reader (Bio-Rad, Hercules, CA). The relative cell viability (\%) was calculated.

\section{TUNEL assay}

To determine the apoptotic cell death, following transduction for $3 \mathrm{~h}$, INS-1E cells were incubated with cytokine cocktail for $48 \mathrm{~h}$. Apoptotic cells were dark stained using DeadEnd Fluorometric TUNEL System from Promega (Madison, WI) as per the manual. To quantitatively determine the effect of XIAP expression on cytokine induced INS-1E cell apoptosis, APO-DIRECT kit (Promega, Madison, WI) was used to detect apoptotic cells. Briefly, following transduction for $3 \mathrm{~h}$ and incubation with the cytokine cocktail for additional $48 \mathrm{~h}$, INS-1E cells were made to single cell suspension by trypsin digestion as described above. The cells were fixed in 1\% paraformaldehyde in PBS (pH 7.4) and then treated with ice-cold 70\% ethanol and stained with FITC-dUTP and propidium iodide. Fluorescent intensity was measured by flow cytometry and analyzed using CELLQUEST software (BD Bioscience). Nontransduced cells without cytokine treatment were served as a negative control. Three sets of independent transduction experiments were carried out for each assay.

\section{Static insulin release}

In vitro function of INS-1E cells and human islets after transduction with AdvhXIAP was determined by the static incubation method. Briefly, after transduction media 
was carefully removed with pipette, INS-1E cells and human islets were sequentially incubated in the media containing $2.5 \mathrm{mM}$ (basal) and $22 \mathrm{mM}$ glucose (stimulated) at $37^{\circ} \mathrm{C}$ for $1 \mathrm{~h}$. Supernatants were collected and analyzed for insulin release by Insulin Ultrasensitive EIA kit (Alpco Diagnostics, Salem, NH). Insulin secretion was expressed as $\mu \mathrm{U} / \mathrm{ml}$ and the ratio of insulin levels at $22 \mathrm{mM}$ to $2.5 \mathrm{mM}$ glucose was used to calculate the stimulation index.

\section{Islet transplantation studies}

Animal experiments were performed as per the NIH (http://grants1.nih.gov/grants/olaw/references/phspol.htm) and institutional animal care and use guidelines using the approved protocol. To induce diabetic animal model, STZ $(40 \mathrm{mg} / \mathrm{kg})$ was administered to NOD-SCID mice by intraperitoneal injection for 5 consecutive days. Animals were considered to be diabetic after two consecutive measurements of blood glucose $\geq 300 \mathrm{mg} / \mathrm{dl}$ using a One Touch Ultra Glucometer (LifeScan, Inc.). Before transplantation, human islets were grouped in four and transduced with blank vehicle, Adv-hXIAP, Adv-LacZ, and Adv-hXIAP with $50 \mathrm{ng} / \mathrm{ml}$ embelin at the dose of 1,000 MOI for $12 \mathrm{~h}$ and washed with PBS. About 1,000 transduced or non-treated islets were transplanted under the left kidney capsules of diabetic mice. The nonfasted glucose levels were measured from the snipped tail of each animal up to 42 days post-transplantation. Then the mice were anesthetized to collect blood to measure serum c-peptide level by ELISA; Alpco Diagnostics (Windham, NH), and the graft-bearing kidneys were removed from some animals to confirm the function of islet grafts by the return of blood glucose levels to $\geq 300 \mathrm{mg} / \mathrm{dL}$ for two consecutive days. Since most mice developed high blood glucose level $\geq 600 \mathrm{mg} / \mathrm{dL}$ after STZ treatment and long time after transplantation, all mice were subjected to daily intraperitoneal administration of $10 \mathrm{U} / \mathrm{kg}$ insulin to maintain mice survival. To minimize the interference to blood glucose measurement by insulin injection, all the measurement were done at least $5 \mathrm{~h}$ after insulin injection.

To determine the effect of ex vivo Adv-hXIAP transduction prior to transplantation on insulin production of human islets post transplantation, transplanted mice were sacrificed at day 43 and kidneys bearing islets were isolated, washed with PBS, fixed in 4\% paraformaldehyde overnight, and embedded in paraffin as described before[12]. Sections of 5-7 $\mu \mathrm{m}$ thickness were cut and immune-stained with rabbit antiinsulin first antibody (Abcam) 1:500 overnight. Then immune-reactivity was detected using goat anti-rabbit IgG, H\& L chain specific peroxidase conjugate (Abcam) and subsequent incubated with DAB substrate. Sildes were further counter-stained Haematoxylin (Sigma). 


\section{Results}

\section{XIAP gene expression in INS-1E cells and human islets}

As shown in Figure 3-2, real-time PCR results showed increase in XIAP mRNA level of INS-1E cells by 3000 folds and 100,000 folds after transduction of INS-1E cells with Adv-hXIAP at MOI of 1 and 10, respectively. Similarly, XIAP protein concentration increased by 13 folds (from $0.54 \mathrm{ng} / \mu \mathrm{g}$ to $6.94 \mathrm{ng} / \mu \mathrm{g}$ ) and 28 folds (from $0.54 \mathrm{ng} / \mu \mathrm{g}$ to $12.59 \mathrm{ng} / \mu \mathrm{g}$ ) for INS-1E cells after transduction with Adv-hXIAP at MOI of 1 and 10. In human islets, dose-dependent XIAP gene expression was also observed after Adv-hXIAP transduction. XIAP protein level increased from the basal level of $16.17 \mathrm{ng} / \mu \mathrm{g}$ to $28.87,75.58$, and $95.55 \mathrm{ng} / \mu \mathrm{g}$ when 1000 IEs were transduced with AdvhXIAP at MOI of 200, 1000, and 2000, respectively. These results were in good agreement with our Western Blot analysis, where XIAP band density increased with increasing MOI, suggesting increase in XIAP protein expression after transduction.

\section{XIAP improves INS-1E cells viability and function against inflammatory cytokines}

Following transduction, INS-1E cells were further incubated with inflammatory cytokine cocktail for $48 \mathrm{~h}$. INS-1E cell viability decreased after cytokine treatment, as determined by the MTT assay (Figure 3-3). However, transduction of INS-1E cells with Adv-hXIAP showed only $14 \%$ decrease in cell viability, compared with untransduced INS-1E cells (37\%) and INS-1E cells transduced with Adv-LacZ (55\%), suggesting the protective effect of XIAP. INS-1E cell function was determined by insulin stimulation test. Following transduction and cytokine treatment for $12 \mathrm{~h}$, INS-1E cells were sequentially incubated with $2.5 \mathrm{nM}$ and $22.5 \mathrm{nM}$ glucose medium and secreted insulin was determined by ELISA. The results showed that under cytokine treatment, AdvhXIAP transduced INS-1E cells did produce more insulin than untransduced INS-1E cells and Adv-LacZ transduced INS-1E cells, suggesting the protective effect of XIAP on INS-1E cell function.

\section{XIAP inhibits INS-1E cell apoptosis against inflammatory cytokines}

One crucial step in apoptosis is DNA fragmentation, resulting from the activation of endonucleases during the apoptotic process [89]. Therefore, the analysis of DNA fragmentation with TUNEL assay is a useful approach to gain information regarding cell apoptosis (Figure 3-4). After 48h of incubation with cytokine cocktail post-transduction, Adv-hXIAP transduced INS-1E cells showed significant decrease in the number of apoptotic cells, which were stained dark brown, compared with untransduced INS-1E cells, and Adv-LacZ transduced INS-1E cells. In a parallel experiment, all cells were fixed, stained with FITC, and run through flow cytometry to count the relative ratio of apoptotic cells. Adv-hXIAP transduced INS-1E cells showed an average of $5.7 \%$ of apoptosis, significantly lower than $27.9 \%$ and $32.8 \%$ of untransduced INS-1E cells and 


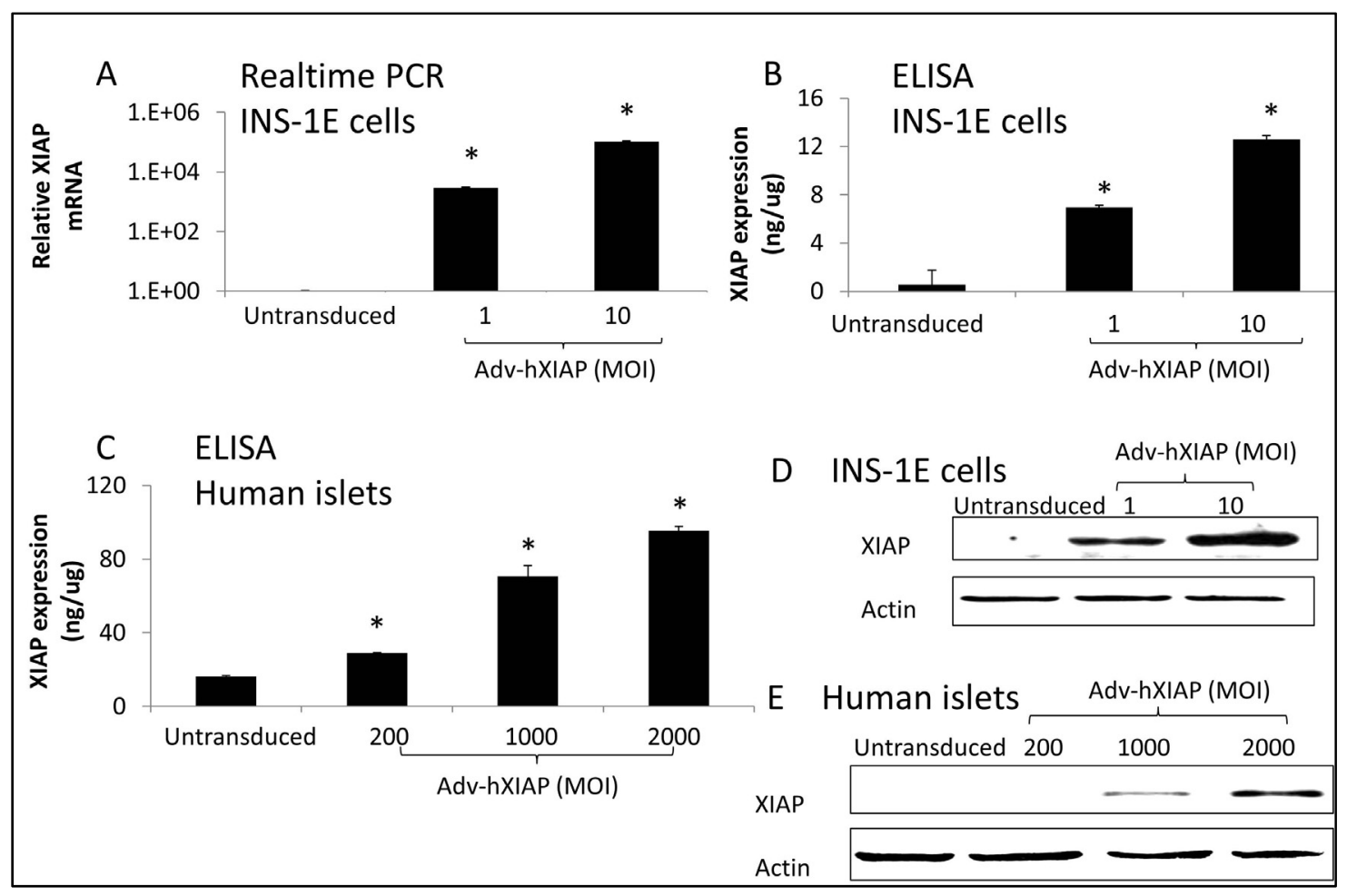

Figure 3-2. Adenovirus mediated overexpression of XIAP in INS-1E cells and human islets

Overexpression of XIAP in $10^{6}$ INS-1E cells and 1000 IEs at 2 days after Adv-hXIAP transduction at indicated MOI as determined by Realtime PCR, ELISA and western blot. Realtime PCR was not done for human islets because of the difficulty of isolating mRNA from islet clusters. MOI describes the number of adenoviral particles capable to transduce each INS-1E cells or each islet cluster. ${ }^{*} p<0.05$ under one way ANOVA compared with untransduced group. Data are presented as the mean + SD. $n=3$. 


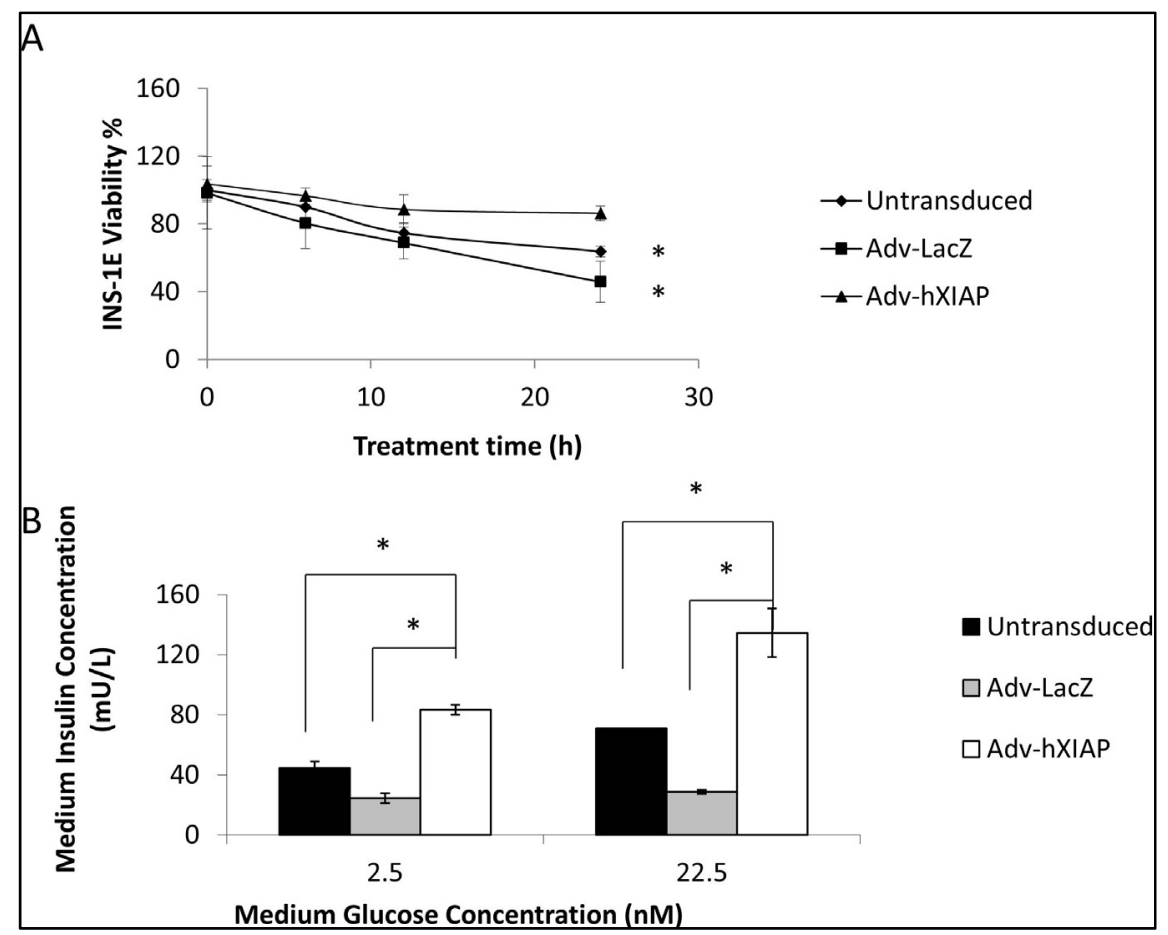

Figure 3-3. Beneficial effect of XIAP overexpression on INS-1E cell viability and function

A. Higher viability of Adv-hXIAP transduced $10^{6}$ INS-1E cells was observed in the presence of inflammatory cytokine cocktail, compared with untransduced cells and AdvLacZ transduced cells. B. More insulin production by INS-1E cells transduced with Adv$\mathrm{hXIAP}$ was observed under cytokine treatment. $\mathrm{n}=3$. Data are presented as the mean \pm SD. ${ }^{*} \mathrm{p}<0.05$ under $\mathrm{t}$ test compared with Adv-XIAP group. 


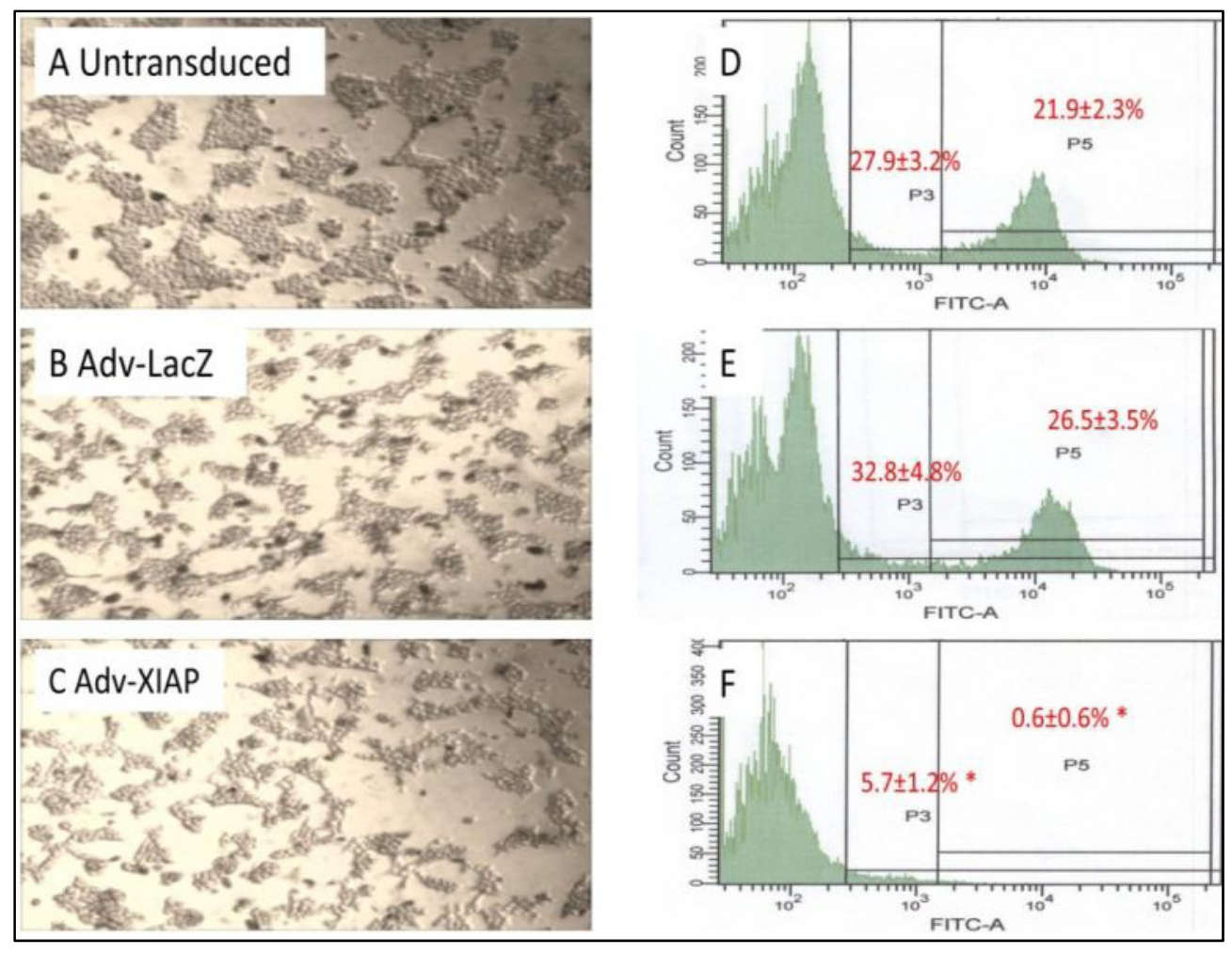

Figure 3-4. Anti-apoptotic effect of XIAP overexpression on INS-1E cells Left panel, dark stain of apoptotic INS-1E cells under cytokine treatment, as determined by DeadEnd Fluorometric TUNEL System. Right panel, apoptotic cell number counted by flow cytometry following fluorescent labeling by APO-DIRECT. The values indicate the percentage of apoptotic cells. A.D. Untransduced INS-1E cells. B.E. Adv-LacZ transduced INS-1E cells. C.F. Adv-hXIAP transduced INS-1E cells. Data are presented as the mean $+\mathrm{SD}$ of $\mathrm{n}=3 .{ }^{*} \mathrm{p}<0.05$ under one way ANOVA compared with untransduced group. 
Adv-LacZ transduced INS-1E cells, respectively.

\section{XIAP inhibits caspase activity in INS-1E cells and human islets}

XIAP binds to caspase 3 and caspase 7 with its BIR 2 domain and caspase 9 with its BIR3 domain [90], and is known to inhibit their activities [91]. Since caspase 3/7 was the converging point of apoptotic pathways, we determined the effect of XIAP gene expression on caspase $3 / 7$ activity. XIAP overexpression led to dose dependent caspase inhibition under cytokine treatment $(5 \mathrm{ng} / \mathrm{ml} \mathrm{IL-1} \beta, 25 \mathrm{ng} / \mathrm{ml} \mathrm{TNF} \alpha$, and $25 \mathrm{ng} / \mathrm{ml}$ IFN $\gamma$ for 3h) (Figure 3-5). Caspase 3/7 activity decreased up to $40 \%$ post transduction of both INS-1E cells and human islets with Adv-hXIAP. Similarly, XIAP expression also inhibited caspase 8 and caspase 9 activities in human islets, resulting in a total inhibition of $45 \%$ and $40 \%$, respectively.

\section{Prolonged normoglycemic control of diabetic mice by Adv-hXIAP transduced human islets}

The effect of XIAP gene expression on the islet survival and function posttransplantation was determined in terms of blood glucose, animal weight gain, and insulin production, by comparing mice transplanted with Adv-hXIAP transduced human islets and mice transplanted with untransduced human islets, Adv-LacZ transduced human islets, and Adv-hXIAP with embelin co-transduced human islets. Following transplantation, blood glucose level decreased to around $200 \mathrm{mg} / \mathrm{dL}$ for all transplanted mice (Figure 3-6), suggesting the immediate reversal of diabetic hyperglycemia by islet transplantation. However, the blood glucose level of the mice transplanted with untransduced islets, Adv-LacZ transduced islets, and Adv-hXIAP plus embelin cotransduced islets gradually increased after the first week post transplantation, and was maintained to the diabetic level ( $\sim 600 \mathrm{mg} / \mathrm{dL})$ since 2 weeks post transplantation (Figure 3-6), suggesting the impaired normoglycemic control caused by the impaired islet viability and function. This result is also consistent with previous reports $[4,8,92]$. In contrast, the mice transplanted with Adv-hXIAP showed prolonged normoglycemic control till 6 weeks post transplantation, probably due to the improvement in transplanted islet viability and function by XIAP overexpression. It should be noted that since most mice became highly diabetic after STZ treatment and the experiment last for more than 6 weeks, all mice were subjected to daily intraperitoneal administration of $10 \mathrm{U} / \mathrm{kg}$ insulin from the second week post transplantation. To minimize the interference of insulin injections to blood glucose measurement, all the measurement were made $5 \mathrm{~h}$ after insulin injection.

As listed in Table 3-1, the mice transplanted with Adv-hXIAP transduced human islets showed significant increase in average body weight six weeks after transplantation, probably due to the prolonged normoglycemic control by XIAP overexpression. To further monitor the long-term islet function following transplantation, the kidney bearing Adv-LacZ transduced islets and Adv-hXIAP transduced islets were isolated at the end of 


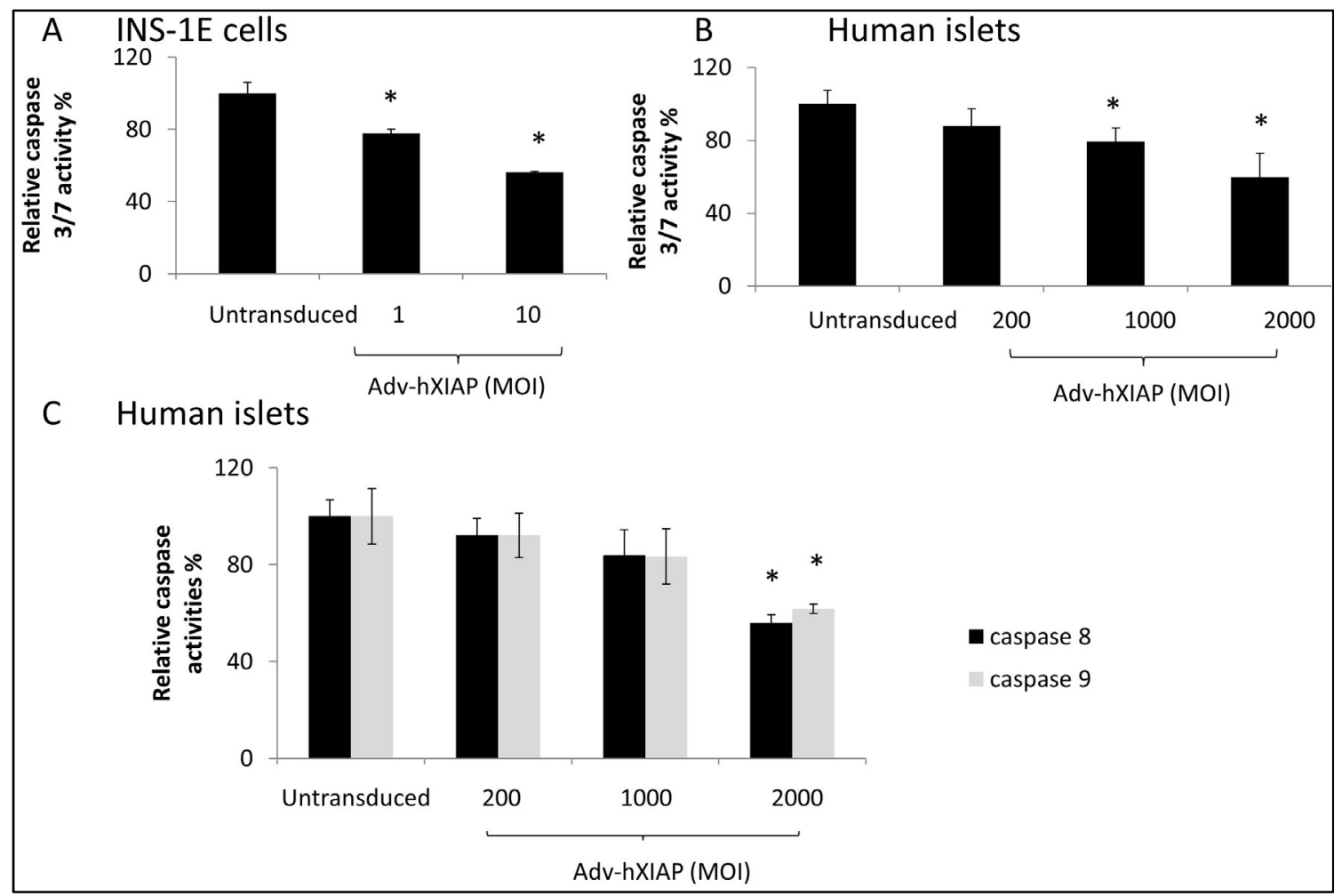

Figure 3-5. XIAP overexpression inhibits caspase activities of INS-1E cells and human islets after cytokine treatment

XIAP expression significantly inhibited activities of caspase $3 / 7,8$, and 9 in a dose dependent manner as determined by Caspase Glo Kit. A. Caspase 3/7 activity in INS-1E cells. B. Caspase 3/7 activity in human islets. C. Caspase 8 and caspase 9 activities in human islets $* p<0.05$ under one way ANOVA compared with untransduced group. Data are presented as the Mean $+\mathrm{SD} . \mathrm{n}=3$. 


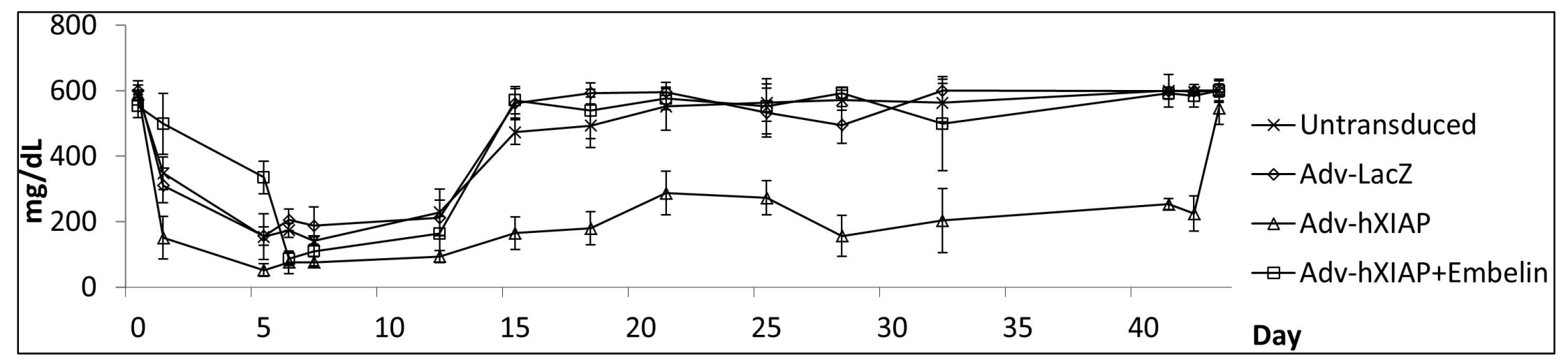

Figure 3-6. Effect of Adv-hXIAP transduction on the outcome of islet transplantation

Blood glucose level of STZ-induced diabetic NOD-SCID mice transplanted with 1000 Adv-hXIAP transduced IEs under the kidney capsule The mice transplanted with Adv-LacZ transduced and nontransduced IE were used as controls. Embelin was also used as negative control since it can reverse the effect of XIAP overexpression. The nonfasting glucose levels were measured in each animal up to 42 days post-transplantation. At day 43 after transplantation, the islet graft-bearing kidney was removed and mice were sacrificed. Data are presented as the Mean \pm SD. $n=5$. 
Table 3-1. Mouse weight measurements after human islet transplantation

\begin{tabular}{lllll}
\hline Weight & Day 0 & Day 7 & Day 28 & Day 42 \\
\hline Control & $18.06 \pm 1.01$ & $17.90 \pm 2.43$ & $18.90 \pm 1.99$ & $19.21 \pm 2.05$ \\
Adv-LacZ & $17.93 \pm 1.33$ & $17.20 \pm 2.35$ & $18.87 \pm 2.28$ & $18.93 \pm 2.40$ \\
Adv-hXIAP & $18.39 \pm 0.69$ & $17.83 \pm 1.85$ & $20.49 \pm 2.36^{*}$ & $22.33 \pm 2.43^{*}$ \\
Adv-hXIAP+Embelin & $18.18 \pm 0.93$ & $18.07 \pm 2.70$ & $18.76 \pm 2.07$ & $19.13 \pm 3.07$ \\
\hline
\end{tabular}

${ }^{*} \mathrm{p}<0.05$ under one way ANOVA test. Data are presented as the Mean \pm SD. $n=5$. 
experiment and subjected to immunohistochemical analysis. The right kidneys without islet transplantation were also isolated and used as the negative control (Figure 3-7). The positive staining of insulin was more evident in kidney section bearing Adv-hXIAP transduced islets, while only rare insulin staining was observed in the kidney section bearing Adv-LacZ transduced islets (Figure 3-7), suggesting the maintenance of insulin secretion. The serum of mice was also collected to measure serum c-peptide level by ELISA. Result showed that c-peptide level in the mice transplanted with Adv-hXIAP transduced islets was significantly higher than the other group, indicating improved islet function in Adv-hXIAP transduced human islets post transplantation (Figure 3-7).

\section{Protective effect of Adv-hXIAP transduction will be reversed by XIAP inhibitor embelin}

As shown in Figure 3-8, embelin cytotoxicity was first determined ex vivo by MTT. Embelin does not show significant toxicity on INS-1E cells in $24 \mathrm{~h}$ treatment when concentration is below $200 \mathrm{nM}$, and $50 \mathrm{nM}$ was selected as a proper concentration of embelin to inhibit XIAP expression. XIAP expression in human islets was greatly inhibited after embelin treatment, even lower than the basal XIAP expression in normal islets, which was consistent with the literature [93, 94]. The cytoprotective effect of XIAP overexpression was impaired by XIAP inhibitor embelin. Adv-hXIAP transduced INS-1E cell viability was decreased to $44 \%$ after incubation with cytokine cocktail and $50 \mathrm{nM}$ embelin for $24 \mathrm{~h}$, compared with $88 \%$ viability with just cytokine cocktail. Caspase $3 / 7$ activity was restored to the untransduced level after embelin treatment, suggesting the inhibitory effect of XIAP gene expression on caspase activity can be reversed by embelin. The prolonged normoglycemic control of diabetic mice by Adv-hXIAP transduced human islets can also be impaired by additional embelin incubation before transplantation, in which the duration of normoglycemia was significantly decreased from 6 weeks to 2 weeks (Figure 3-6), suggesting the loss of islet viability and function by XIAP inhibition.

\section{Discussion}

Human islet transplantation has great potential to provide type 1 diabetic patients with sustained and improved normoglycemic control and a period of insulin independence. However, most islet grafts get destroyed in the early days posttransplantation due to inflammatory cytokines, ROS and failure to revascularize induced by immune rejection, let alone the islet loss in islet isolation, transportation and transplantation processes $[95,96]$. Although the use of immunosuppressant to the transplant recipient can partially relieve graft rejection by the host immune system, these drugs have significant toxicity including nephrotoxicity, ulcers, hyperglycemia, osteoporosis, and increased risk of infection and neoplasms [97-100]. Therefore, islets from two to four donors are generally necessary for successful islet transplantation [101]. Since the limited islet supply nowadays cannot meet the increasing islet need for transplantation, improving islet graft viability and function post transplantation is one of 


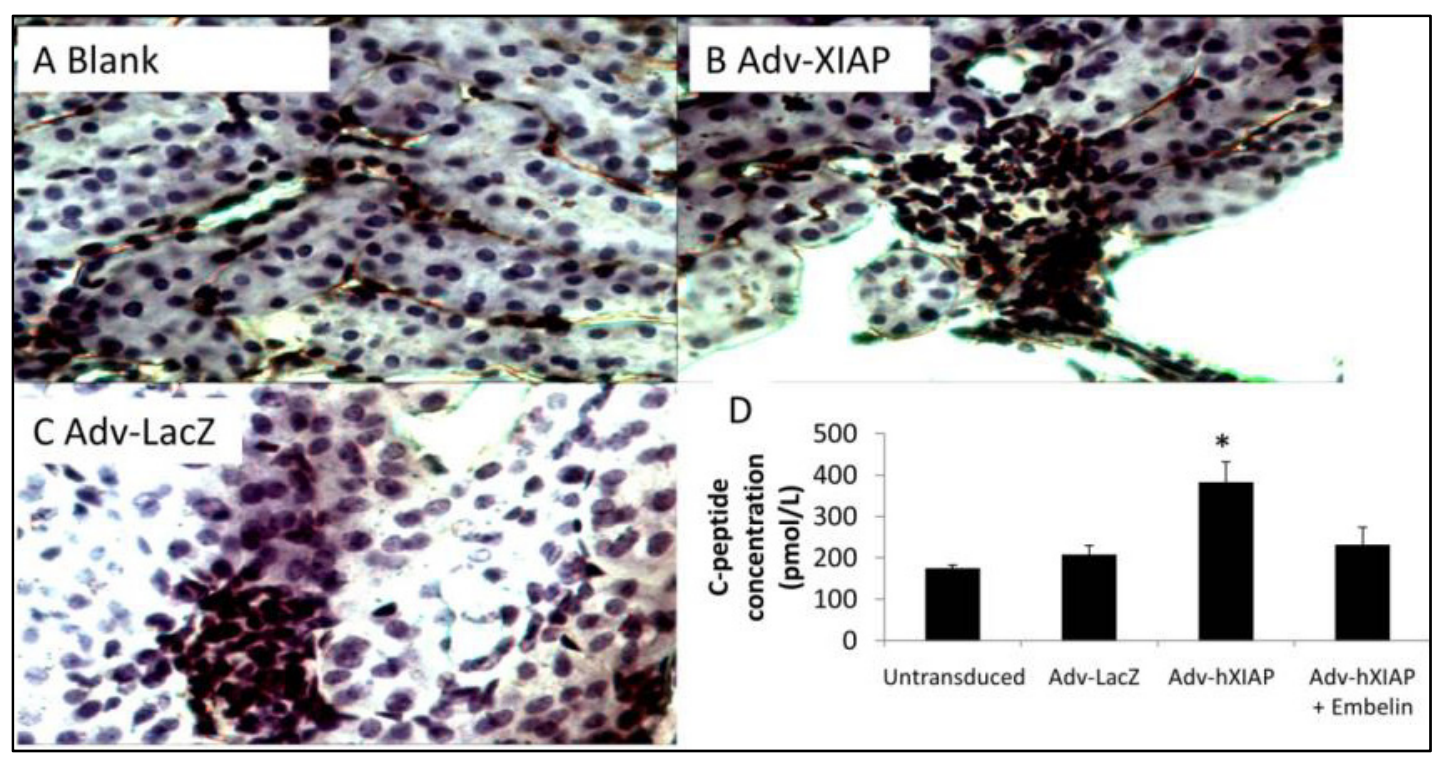

Figure 3-7. Immunohistochemical staining of kidney sections at 42 days after transplantation

A. Kidney section bearing no islet graft. B. Kidney section bearing Adv-hXIAP transduced islet grafts. C. Kidney section bearing Adv-LacZ transduced islet grafts. Insulin was stained in brown color. Nuclear was stained in purple by haematoxylin. D. Increasing C-peptide level in Adv-hXIAP group was observed in the serum collected at 42 days after transplantation. ${ }^{*} \mathrm{p}<0.05$ under one way ANOVA compared with untransduced group. Data are presented as the Mean \pm SD. $n=5$. 


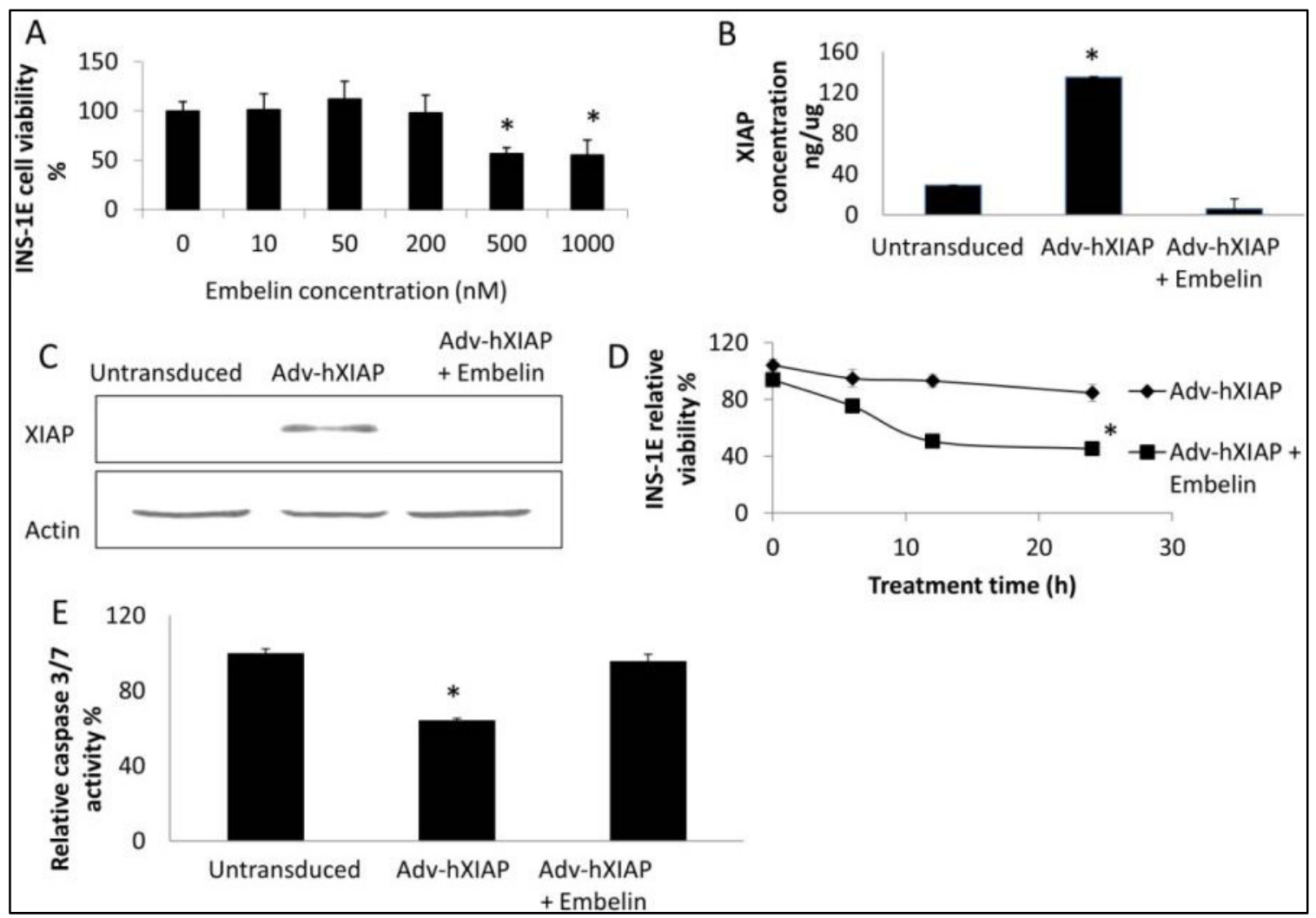

Figure 3-8. Beneficial effect of XIAP overexpression on INS-1E cells can be reversed by XIAP inhibitor embelin

A. Cytotoxicity study of embelin on $10^{6}$ INS-1E cells/well for 24 h. B-C. Embelin can inhibit XIAP expression as illustrated by ELISA and western blot. D. Embelin reversed the cytoprotective effect of XIAP overexpression on $10^{6}$ INS-1E cells against cytokine treatment. E. Embelin reversed the caspase inhibition effect of XIAP overexpression on INS-1E cells. ${ }^{*} p<0.05$ under one way ANOVA compared with zero embelin concentration. Data are presented as the Mean \pm SD. $n=3$. 
the major barriers to overcome for successful islet transplantation.

As we mentioned before, the first two weeks post islet transplantation is crucial for graft survival. This makes adenoviral vector a good candidate for ex vivo gene therapy because of its high transduction efficacy and stable expression ability up to eight weeks $[102,103]$. In our group, we have been working on the adenovirus mediated gene therapy method to improve the islet viability and function against graft rejection post transplantation for years. Briefly, besides the growth factor gene delivery to reduce apoptotic $\beta$-cell death and promote $\beta$-cell proliferation post transplantation, we also demonstrated that proapoptotic gene silencing such as iNOS silencing and caspase 3 silencing by siRNA and shRNA respectively, could reduce the apoptotic $\beta$-cell death caused by inflammatory cytokines $[5,6,8,17]$.

Compared with other gene therapy methods, XIAP gene delivery holds its own merits. The major problem with growth factor gene therapy was that growth factors were secreted protein, whose effects were determined by microenvironment concentration. Therefore, in Adv-transduced human islet clusters, growth factor concentration in extracellular microenvironment could not be uniformly distributed and finely regulated. Accumulation of microenvironmental VEGF in the outer layer of human islets may finally lead to aberrant angiogenesis and islet loss [10, 104].

XIAP is strictly localized in the cytoplasm in a granular supranuclear pattern [105]. Therefore, after Adv-hXIAP transduction, the outer layer of human islets which will be more exposed to the inflammatory cytokines and hypoxic environment, will express higher level of XIAP and achieve an optimal defense mechanism against cytokine and hypoxia challenge in the early stage of graft survival. Transduction of INS$1 \mathrm{E}$ cells and human islets with Adv-hXIAP resulted in significant increased XIAP expression (Figure 3-2). With adenovirus mediated XIAP overexpression method, we can easily reach the caspase inhibition effect in human islets without inducing acute immune response by ex vivo transduction. Adv-hXIAP ex vivo transduction also led to $\sim 40 \%$ inhibition of caspase 3/7, caspase 8, and caspase 9 activities in both INS-1E cells and human islets (Figure 3-5). The protective effect of Adv-hXIAP transduction on human islets was lasted for more than six weeks after transplantation. Another advantage of XIAP expression, though not discussed in this paper, is its ability to reverse the islet loss caused by hypoxia $[16,106]$, which made it a good candidate for ex vivo transduction of islets prior to transplantation.

As a result of caspases inhibition, we also observed the protective effect of XIAP expression on INS-1E cells against cytokine cocktail treatment. Compared with untransduced INS-1E cells, Adv-XIAP transduced INS-1E cells showed significant improved cell viability, decreased apoptotic cells and increased insulin production under cytokines treatment. One interesting point we observed in this experiment is that AdvLacZ transduced INS-1E cells showed decreased cell viability and decreased insulin production under cytokines treatment, despite the fact that Adv-LacZ at such low MOI $(\mathrm{MOI}=2)$ has no significant effect on INS-1E cell viability (data not shown), suggesting the cytotoxic or proapoptotic effect of inflammatory cytokines could be possibly 
exaggerated by viral transduction. But since XIAP expression effectively blocked the caspases activation, it reversed the cytotoxic or proapoptotic effect of cytokines.

Our in vivo data showed the prolonged normoglycemia control of highly diabetic NOD-SCID mice and increasing insulin production after transplantation with AdvhXIAP transduced human islets for six weeks, which was much longer than the previous reports $[8,17,86]$, probably due to the protective effect of XIAP in the first two weeks after transplantation. However, when transplanted with human islets in which XIAP expression was almost silenced by co-culturing with its inhibitor embelin, the blood glucose level of the diabetic NOD-SCID mice was decreased (Figure 3-6). This result suggested XIAP level played a crucial role in determining islet viability after transplantation in highly diabetic patients. However, it should also be addressed that all mice were subjected to daily intraperitoneal administration of $10 \mathrm{U} / \mathrm{kg}$ insulin from the second week post transplantation because it was necessary to keep the mice survival in all the other three groups except the one transplanted with Adv-hXIAP transduced human islets or these mice might not have survived for six weeks with such high blood glucose levels. To minimize the interference of insulin injection to blood glucose measurement to the lowest extent, all the measurement were made at least $5 \mathrm{~h}$ after insulin injection.

Adv-hXIAP transduced INS-1E cells and human islets, when exposed to small molecular XIAP inhibitor embelin, had greatly decreased XIAP activity and therefore decreased cell viability and insulin production under inflammatory cytokines. Our data suggest that embelin inhibited XIAP activity by blocking protein synthesis, which is in good agreement with the previous report that embelin regulated apoptotic related gene expression [107], not by structure based protein-protein interaction as described before [93]. Embelin could inhibit XIAP expression to an extremely low level which was even lower than the basal XIAP level in normal INS-1E cells and human islets, and hence reversed the protective effect of XIAP in INS-1E cells and human islets against cytokine, suggesting the important role of XIAP expression in graft viability and function post transplantation.

The therapeutic range of Adv-hXIAP transduction is very hard to determine. The MOI of Adv vector used for transduction must be carefully selected to achieve optimal transduction efficacy, with little toxicity. Our data showed that Adv-hXIAP could elevate XIAP expression in human islets in a dose dependent manner (Figure 3-2) without significant toxicity, and this result was in good agreement with previous reports $[4,6,8$, $16,17]$.

Taken together, these data confirm that XIAP overexpression in human islets enhances islet viability and function against inflammatory cytokines and in doing so helped to achieve prolonged normoglycemic control in diabetic mice, suggesting that clinical application of this protocol could immediately and greatly enhance the availability and long-term outcome of islet transplantation for type I diabetes. 


\section{CHAPTER 4. RGD MODIFIED ADENOVIRUS ENCODING XIAP AND HGF TO SIMULTANEOUSLY PREVENT EARLY GRAFT APOPTOSIS AND PROMOTE REVASCULARIZATION TO IMPROVE THE OUTCOME OF HUMAN ISLET TRANSPLANTATION*}

\section{Introduction}

In the previous study, we get a proof of concept that anti-apoptotic gene delivery effectively prevented the early graft apoptosis of human islet transplantation. However, several issues were unsolved.

First, the transduction efficiency of traditional adenovirus to human islet is low therefore a high MOI is needed to achieve sufficient transgene expression. In the previous study, to achieve optimal XIAP expression, human islet was transduced with Adv-XIAP at MOI 2000. A high MOI could lead to two unwanted outcomes, difficulties in washing off the free viral particles and the coexpression of a significant portion of viral proteins, which raised safety concerns of adenovirus-based gene therapy and increased the immunogenicity of human islets, respectively. The low transduction efficiency and high MOI of traditional adenovirus called for the construction of more efficiency vectors.

Second, anti-apoptotic gene transfer is incapable to assist the revascularization of human islets. Insulin producing $\beta$ cells in human islet need anti-apoptotic gene expression to overcome the challenges after transplantation. They also need supplies of oxygen and nutrition as well as the channels to export the insulin. Failure to revascularization led to hypoxia and starvation in human islets and subsequently caused a significantly portion of islet loss after transplantation. Previous studies demonstrated that a functional revascularization in the first two weeks after human islet transplantation is critical to the survival and engraftment of transplanted islets [17]. Therefore, co-expression of antiapoptotic proteins with angiogenesis factors could be a reasonable strategy to address this issue.

Last but not the least, the diabetic animal model we used for human islet transplantation needed immediate improvements because most mice became overdiabetic with current protocol of streptozotocin injection. Insulin must be injected to maintain the euglycemia besides the human islet transplantation which significantly clouded the outcome of transplantation surgery and the results of functional analysis of transplanted islets. A better protocol to induce diabetes in NOD-SCID mice was needed to generate moderate level of diabetes, which is similar to clinical cases and reversible by the islet transplantation only. By doing this, we will have an important standard to

\footnotetext{
* Reprinted with permission from John Wiley \& Sons. Wu, H, Yoon, AR, Li, F, Yun, CO, and Mahato, RI (2011). RGD peptide-modified adenovirus expressing hepatocyte growth factor and $\mathrm{X}$-linked inhibitor of apoptosis improves islet transplantation. The Journal of Gene Medicine 13: 658-669.
} 
determine whether islet transplantation is successful or not by rendering the diabetic animals insulin-independent, an important clinical criterion of human islet transplantation.

Therefore, in this study we tried to improve the traditional gene therapy on human islet transplantation from three aspects. First, we inserted an Arg-Gly-Asp (RGD) peptide expression cassette into the adenoviral genome encoding HI fiber knob so that an RGD modified fiber knob can be expressed on the surface of viral particles. We hypothesized this RGD modified adenovirus would have high transduction efficiency against human islets because RGD was known to bind with high affinity to several types of integrins present on the surface of mammalian cells. Second, instead of using adenovirus with one transgene insertion within E1 deletion, we used adenovirus with two inserts (XIAP and HGF) within E1 and E3 deletions. We hypothesized co-expression of anti-apoptotic gene and angiogenic factor would have significant advantage in the outcome of human islet transplantation to the previous studies in which one gene was expressed at a time. Third, we revaluated the methodology to generate diabetic mice and found out that the manner of STZ injection had great impact on the extent of diabetes. Because the optimal blood glucose level was within 300 500mg/dL for human islet transplantation, we developed a new manner of STZ injection so that $>80 \%$ of mice will develop an optimal level diabetes which can be reversed to insulin-independence by the islet transplantation only.

\section{Materials and Methods}

\section{Culturing islets}

Human islet preparations were received from the Integrated Islet Distribution Program funded by the National Institute of Diabetes and Digestive and Kidney Diseases (NIDDK) and with support from the Juvenile Diabetes Research Foundation International (JDRFI). Upon arrival, islet preparations were subjected to quality control assessment for viability by using calcein $\mathrm{AM}$ /propidium iodide staining and for purity by using dithizone staining. Dithizone binds zinc ions present in the beta cells of islets, and therefore stains the islets red. Other exocrine tissue also present in the preparations does not bind dithizone, and is therefore not stained[108]. Islet preparations with purity and viability $>$ 90\% were cultured in CMRL-1066 medium (Sigma Aldrich, St. Louis, MO) containing $10 \%$ FBS (Gibco, Gaithersburg, MD) in a $37^{\circ} \mathrm{C}$ incubator.

\section{Construction of RGD-modified Adv}

RGD-Adv-GFP was a kind gift from Dr. Hiroyuki Mizuguchi, Osaka University, Japan. Adv-GFP was constructed and passaged in our lab. To generate RGD-modified Adv, the genome encoding the fiber in Ad5 (from 28592 to 30470) was cloned into the SacII-KpnI site of pBluescript II SK(+) (Stratagene, La Jolla, CA), creating pSK5543, and the genome encoding the fiber in Ad5 (from 32123 to 32836) was cloned into the EcoRI-HindIII site of pSP72 (Promega, Madison, WI), creating pSP72(713). For ease of 
incorporating the RGD epitope between the HI-loop (LNVTQETGDTTPSAYSMSFSWD) of the fiber knob, immediately downstream of the following the 11th threonine, new BamHI and MroI sites were added by PCR-mediated site-directed mutagenesis. For PCR-mediated site-directed mutagenesis, the following primer set was used: 5'- GAA ACA GGA GAC ACA GGA TCC GCG TCC GGA ACT CCA AGT GCA TAC -3' as the sense primer and 5'- GTA TGC ACT TGG AGT TCC GGA CGC GGA TCC TGT GTC TCC TGT TTC -3' as the antisense primer. After PCR amplification, the resulting mutated PCR product, containing BamHI and MroI sites (underlined) to make pSP72 [713-BM], was verified using an ABI PRISM 337 automatic DNA sequencer (Applied Biosystems, Foster City, CA). To construct a vector encoding the RGD epitope between the HI-loop of the fiber knob, two complementary oligonucleotides were synthesized and annealed to form a DNA duplex. This DNA duplex was designed to contain a BamHI overhang 5' end and an MroI overhang 3' end, so that the fragment could be inserted into the BamHI and MroI sites of the pSP72[713$\mathrm{BM}$ ] vector. The RGD epitope consisted of a nine-amino acid sequence, CDCRGDCFC. Sequences of the oligonucleotides were 5'- GA TCC TGT GAC TGC CGC GGA GAC TGT TTC TGC T -3' and 5'- CC GGA GCA GAA TCA GTC TCC GCG GCA GTA ACA G-3'. These oligonucleotides encoded the RGD epitope (boldface and italicized). The resulting plasmid was then digested with NcoI and MfeI and cloned into pSK5543 to generate an Adv fiber shuttle vector, pSK[5543-RGD]. To generate the RGD-modified Adv, the newly constructed Adv fiber shuttle vector pSK[5543+RGD] was digested with SacII and XmnI, and the viral vector d1324 was digested with SpeI for homologous DNA recombination in E. coli BJ5183.

\section{Generating RGD-Adv-hHGF-hXIAP}

To generate an Adv encoding HGF and XIAP at the E1 and E3 regions, respectively, we first constructed an E3 shuttle vector expressing XIAP. The XIAP gene was cloned from pSport6-XIAP by using 5'-CG GGATCC ATG ACT TTT AAC AGT TTT GAA GGA TCT AAA-3' and 5'-CG GGATCC TTA AGA CAT AAA AAT TTT TTG CTT GAA AGT-3' primers and subcloned into the Adv E3 shuttle vector, pSP72E3[109], generating a pSP72-XIAP. The newly constructed pSP72-XIAP shuttle vector was linearized with XmnI digestion and then co-transformed with a replicationincompetent Adv, dl324-RGD, into E. coli BJ5183. It was then digested with SpeI for homologous recombination, yielding Adv-encoding plasmid, d1324-XIAP (E3)-RGD. Structures of the resulting recombinant vectors were then confirmed by restriction enzyme digestion and PCR analysis.

In addition, to construct an Adv E1 shuttle vector expressing HGF, HGF gene was excised from pCDNA3.1-HGF by using BamHI and XbaI and subcloned into the replication-incompetent E1 shuttle vector, pCA14 (Microbix, Ontario, Canada). Then, the Adv E1 shuttle vector was linearized with XmnI digestion and co-transformed into E. coli BJ5183 with the BstBI-digested d1324-XIAP-RGD for homologous recombination, generating d1324-HGF-XIAP-RGD. Recombinant Adv encoding plasmid was digested with PacI and transfected into 293 cells to generate the replication-incompetent Adv, 
RGD-Adv-hHGF-hXIAP (Figure 4-1). The propagation, purification, titration, and quality analysis of all Adv used were performed as previously described [110].

\section{Transduction efficiency}

Human islets were first transduced with Adv-GFP and RGD-Adv-GFP at equal MOI to determine the effect of RGD modification on the transduction efficiency of Adv. To determine the optimal MOI for RGD-Adv-hHGF-hXIAP, 500 hand-picked human islets were transduced with serial dilutions of RGD-Adv-hHGF-hXIAP in 24-well plates for $12 \mathrm{~h}$ and incubated for additional $48 \mathrm{~h}$. Medium were collected. Human islets were washed with PBS and dispersed into a single-cell suspension by incubation with $0.25 \%$ trypsin/EDTA (Gibco, Gaithersburg, MD) at $37^{\circ} \mathrm{C}$ for $5 \mathrm{~min}$ followed by passage through a narrow-gauge pipette. Total protein was extracted with RIPA buffer (Sigma Aldrich, St. Louis, MO) and stored at $-80 \mathrm{oC}$. HGF concentration in the cultured medium and XIAP concentration in the total protein extract of human islets was measured by ELISA (R\&D Systems, Minneapolis, MN).

\section{Caspase detection}

Caspase-Glo 3 assay kits were used to analyze caspase 3 activities as described before [6]. Briefly, $48 \mathrm{~h}$ following transduction, a single-cell islet suspension was generated by $0.25 \%$ trypsin/EDTA digestion. Then, $100 \mu$ l Caspase-Glo were added to $100 \mu \mathrm{l}$ of the single-cell suspension of 104 cells in 96-well plates and incubated at room temperature for $1 \mathrm{~h}$. The contents were then transferred to culture tubes, and luminescence was determined using a luminometer (Berthold, Germany).

\section{Western blot}

Five hundred human islets were transduced with RGD-Adv-hHGF-hXIAP at 500 MOI in 24-well plates for $12 \mathrm{~h}$ and incubated for additional $48 \mathrm{~h}$. A single-cell islet suspension was generated by $0.25 \%$ trypsin/EDTA digestion. The total protein samples were extracted with RIPA buffer supplemented with a protease inhibitor cocktail (Sigma Aldrich, St. Louis, MO), mixed with $6 \times$ Laemmli sodium dodecyl sulfate (SDS) buffer (Boston BioProducts) and then boiled for $5 \mathrm{~min}$ to denature the protein. Then, a $20 \mu \mathrm{g}-$ sample was loaded onto a 4\%-15\% Tris- $\mathrm{HCl}$ precast polyacrylamide gel (Bio-Rad, Hercules, CA) for electrophoresis and subsequently transferred to an immobilon polyvinylidene fluoride (PVDF) membrane (Millipore, Billerica, MA). After blocking with 3\% BSA in $1 \times$ PBST (PBS containing $0.05 \%$ Tween-20) for $1 \mathrm{~h}$ at room temperature, the membranes were incubated with goat polyclonal IgG to XIAP, BAX, BCL-2, p-AKT, AKT and GADPH (Abcam, Cambridge, MA) primary antibodies (1:500) overnight at $4^{\circ} \mathrm{C}$. The membrane was then incubated with HRP-conjugated rabbit polyclonal antibody to goat (1:10000) (Abcam, Cambridge, MA) for $1 \mathrm{~h}$ at room temperature. Target proteins were detected by enhanced chemiluminescence (ECL) 


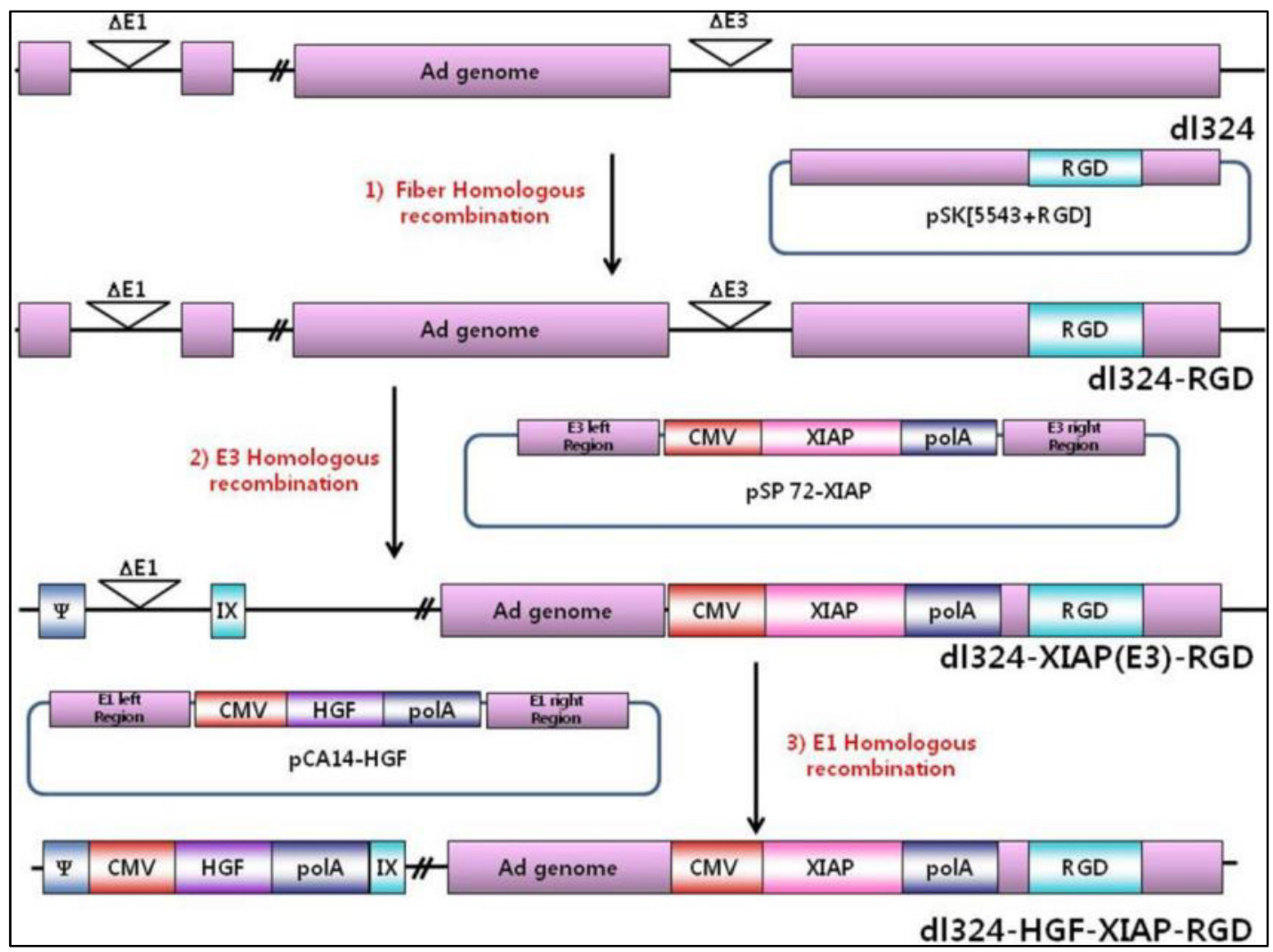

Figure 4-1. Construction of RGD modified Adv genome encoding HGF and XIAP in $\mathrm{E} 1$ and $\mathrm{E} 3$ deletion

RGD epitope expressing cassette, hXIAP cDNA and hHGF cDNA were sequentially incorporated into the Ad5 genome by homologous DNA recombination. 
detection kit (GE Healthcare Life Sciences, Pittsburgh, PA).

\section{Apoptosis study}

A cytokine cocktail of recombinant $\mathrm{TNF} \alpha(5 \mathrm{ng} / \mathrm{ml})$ and IL-1 $\beta(5 \mathrm{ng} / \mathrm{ml})$ was used to mimic the in vivo challenge to the INS-1E cells and human islets by the inflammatory cytokines. INS-1E cells were transduced with Adv for $3 \mathrm{~h}$, stimulated with cytokine cocktail for an additional $48 \mathrm{~h}$ and characterized by DeadEnd ${ }^{\mathrm{TM}}$ Colorimetric TUNEL system (Promega, Madison, WI), in which fragmented DNA from apoptotic cells was labeled with biotinylated nucleotide and detected using hydrogen peroxide and diaminobenzidine (DAB). Human islets were transduced with Adv for $12 \mathrm{~h}$ and incubated with cytokine cocktail for an additional 3 days. Then islet cells were digested with $0.25 \%$ trypsin/EDTA into a single-cell suspension, stained with Annexin V-FITC Apoptosis Detection Kit (Abcam, Cambridge, MA) and analyzed with flow cytometry. Annexin V binds to phosphatidylserine on the cell surface, which is a feature found in apoptotic cells. Fluorescent intensity was analyzed using CELLQUEST software (BD Bioscience, Franklin Lakes, NJ). Nontransduced cells without cytokine treatment served as a negative control. Three sets of independent transduction experiments were carried out for each assay.

\section{Dynamic insulin release}

We designed and constructed an islet perifusion set for dynamic analysis of the function of human islets (Figure 4-2). Compared with static insulin release method, this method gives a real-time monitoring of the islet function against difference stimuli. Briefly, after being stimulated with a cytokine cocktail of IL-1 $\beta(5 \mathrm{ng} / \mathrm{ml})$ and TNF- $\alpha(5$ $\mathrm{ng} / \mathrm{ml}$ ) for 3 days, 50 islets from each group were handpicked and loaded onto a Swinnex 13 chamber (Millipore, Burlington, MA) and perifused with Krebs Ringer-bicarbonate (KRB) buffer containing basal glucose $(2.5 \mathrm{mM})$. The flow rate was maintained at 2 $\mathrm{ml} / \mathrm{min}$ with a peristaltic pump (Themo Fisher, Waltham, MA) and the temperature was maintained at $37^{\circ} \mathrm{C}$ with a solution heater (Warner Instruments, Hamden, CT). Islets were first perifused with basal glucose $(2.5 \mathrm{mM})$ for $45 \mathrm{~min}$ and stimulated with glucose $(22.5 \mathrm{mM})$ for $15 \mathrm{~min}$ and at last perifused with basal glucose $(2.5 \mathrm{mM})$ until insulin release reversed to basal level. Samples were collected through a fraction collector (Waters, Milford, MA) and analyzed for insulin content by ELISA (Calbiotech, Spring Valley, CA).

\section{Generate moderate diabetic nod-scid mice}

To find out the best protocol to induce diabetes in NOD-SCID mice with moderate extent of blood glucose levels for human islet transplantation, several manners and doses of STZ injection were tested. In the first protocol, two shots of STZ were injected intraperitoneally into NOD-SCID mice at a dose $70 \mathrm{mg} / \mathrm{kg}$ for two consecutive 


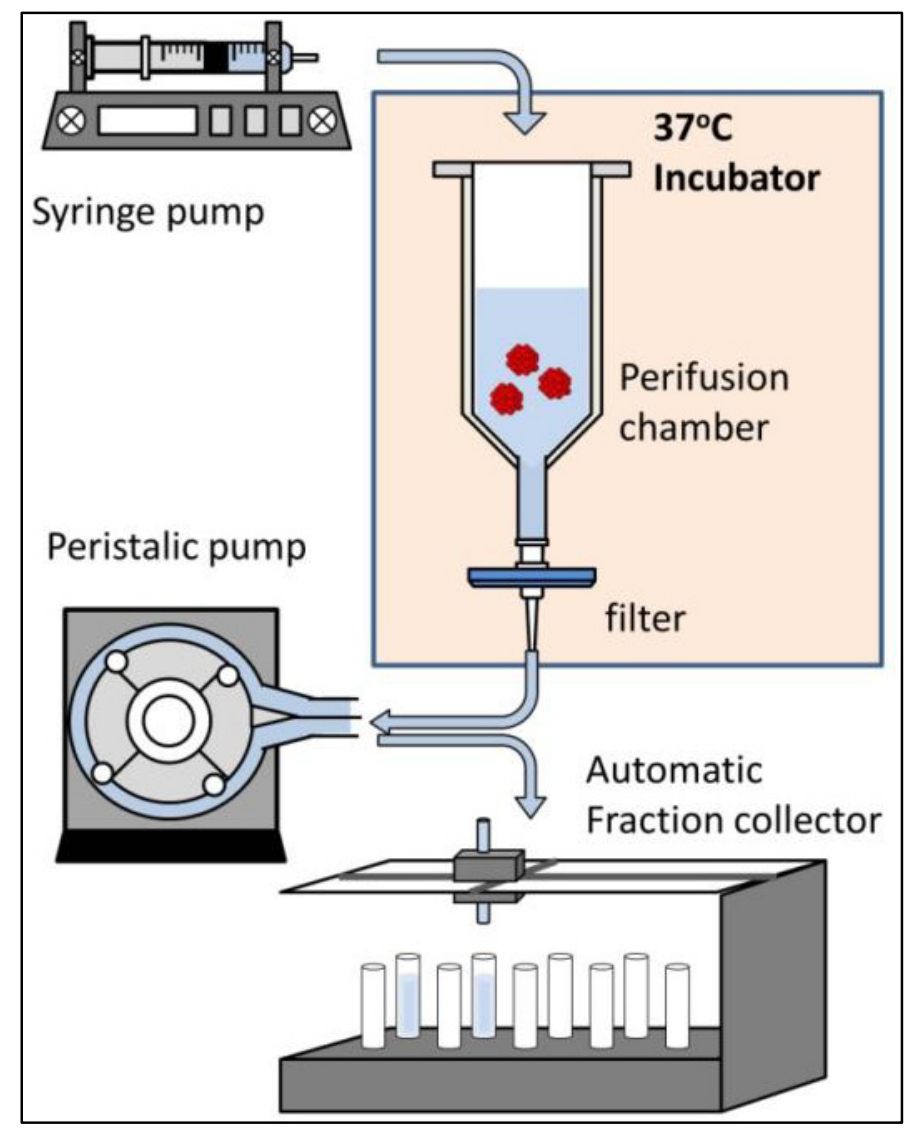

Figure 4-2. Islet perifusion set for dynamic analysis of function of human islets 
weeks. If the mice developed moderate level of diabetes (blood glucose $300 \sim 500 \mathrm{mg} / \mathrm{dL}$ ) in the first week, the second shot can be skipped. In the second protocol, one shot of STZ was injected intraperitoneally into NOD-SCID mice at a fixed dose $150 \mathrm{mg} / \mathrm{kg}$. In the third protocol, STZ was injected intraperitoneally into NOD-SCID mice at a dose of 40 $\mathrm{mg} / \mathrm{kg}$ for 5 consecutive days. The levels of blood glucose of all mice were determined by a glucometer at 3 weeks after last STZ injection. A boxplot of the minimum, the 10 percentile, the median, the 90 percentile, and the maximum was draw for each protocol. Since the measurable glucose concentration of our glucometer is $50 \sim 600 \mathrm{mg} / \mathrm{dL}$, if a measurement gives a reading $\mathrm{HI}$ (high) or LOW, it would be recorded as $600 \mathrm{mg} / \mathrm{dL}$ and $50 \mathrm{mg} / \mathrm{dL}$ respectively.

\section{Islet transplantation}

Animal experiments were performed by approved protocol following NIH and institutional animal care and use Institutional Animal Care and Use Committee guidelines using an approved protocol. To induce diabetes, STZ $(70 \mathrm{mg} / \mathrm{kg}$ ) was administered to NOD-SCID mice by intraperitoneal injection for 2 consecutive days. Animals were classified as diabetic after two consecutive measurements of blood glucose $\geq 400 \mathrm{mg} / \mathrm{dl}$ with a glucometer. Upon receiving human islets from the Integrated Islet Distribution Program (IIDP) distribution centers, they were immediately transduced with RGD-AdvhHGF-hXIAP at $500 \mathrm{MOI}$ for $12 \mathrm{~h}$. Then islets were washed three times to remove free virus. Then 500 islets were carefully hand-picked and transplanted under the kidney capsule of each STZ-induced diabetic NOD-SCID mouse. Three shots of insulin $(5 \mathrm{U} / \mathrm{kg})$ were given to each mouse on the first 3 days after transplantation to relieve the hyperglycemic stress to the newly transplanted islets and no insulin was given afterwards. The nonfasted glucose levels of all the mice were measured from the snipped tail of each animal at 2 p.m. during the first week after surgery and then weekly. At the end of study, the mice were anesthetized to collect blood to measure serum insulin and c-peptide levels by ELISA. The graft-bearing kidneys were then removed from some animals to confirm the function of islet grafts by the return of blood glucose levels to $\geq 400 \mathrm{mg} / \mathrm{dL}$.

\section{Intraperitoneal glucose tolerance test}

Thirty days after islet transplantation, glucose tolerance was determined in overnight-fasted mice as described by Garcia-Ocana et al. [9]. Briefly, the mice were subjected to intraperitoneal injection of glucose at $2 \mathrm{~g} / \mathrm{kg}$ of body weight. Blood samples were obtained from the snipped tail at 15, 30, 60, 90 and 120 min after injection and analyzed for glucose levels with a glucometer.

\section{Immunofluorescence staining}

In an independent study, 500 hundred handpicked islets were transplanted under the kidney capsule of STZ-induced diabetic NOD-SCID mice. Six mice from each group 
(mice receiving untransduced human islets and mice receiving RGD-Adv-hHGF-hXIAP transduced human islets at $500 \mathrm{MOI}$ ) were sacrificed 30 days after transplantation.

Another six from each group was sacrificed at 200 days after islet transplantation. The kidneys bearing islets were isolated, washed with PBS, fixed in 4\% paraformaldehyde overnight, and embedded in optimal cutting temperature compound. Frozen sections of $10 \mu \mathrm{m}$ thickness were cut. To detect insulin-positive human islets, the slides were stained with guinea pig anti-insulin primary antibody $(1: 200)$ at $4{ }^{\circ} \mathrm{C}$ overnight and Alexa Fluor 568-conjugated goat anti-guinea pig secondary antibody (1:500) at room temperature for $1 \mathrm{~h}$. To detect revascularization, the slides were stained with rabbit anti-von-Willebrand factor (vWF) primary antibody $(1: 500)$ at $4{ }^{\circ} \mathrm{C}$ overnight and Dylight 488 -conjugated goat anti-rabbit secondary antibody (1:500) at room temperature for $1 \mathrm{~h}$. Slides were counterstained with DAPI.

\section{Results}

\section{Construction of RGD-Adv-hHGF-hXIAP}

To improve the transduction efficiency of Adv on human islets, we chose to introduce an RGD peptide, which is known to bind with high affinity to several types of integrins present on the surface of mammalian cells, into the HI loop of the fiber knob. An RGD-modified Adv was generated by sequentially incorporating and RGD epitopeexpressing cassette, hXIAP cDNA and hHGF cDNA, into the genome of adenovirus serotype 5 through homologous DNA recombination (Figure 4-1). Structures of the resultant recombinant vectors were then confirmed by restriction enzyme digestion and PCR analysis. A proper amount of recombinant plasmid was digested with PacI and transfected into 293 cells, thereby generating the replication-incompetent Adv, RGDAdv-hHGF-hXIAP.

\section{Transduction efficiency of RGD-Adv-hHGF-hXIAP on human islets}

The transduction efficiency of traditional Adv-GFP and RGD-Adv-GFP was compared. Results showed that the RGD modification increased the expression of GFP in human islets, suggesting improved transduction efficiency (Figure 4-3). Optimal MOI of RGDAdv-hHGF-hXIAP was determined by transducing human islets with serial dilutions of RGD-Adv-hHGF-hXIAP. Results showed that HGF and XIAP expression were upregulated in a dose-dependent manner. At 500 MOI, RGD-Adv-hHGF-hXIAP could upregulate HGF and XIAP to the extent usually requiring MOI $>2000$ for traditional Adv $[8,111]$. The gene expression profile of RGD-Adv-hHGF-hXIAP-transduced human islets over 2 weeks indicated the transient manner of Adv-mediated gene expression. The amount of HGF and XIAP produced by RGD-Adv-hHGF-hXIAP transduced human islets peaked 2 days after transduction and decreased gradually in the following 14 days. However, the levels of HGF and XIAP were still much higher compared with the endogenous expression of these two therapeutic genes. 


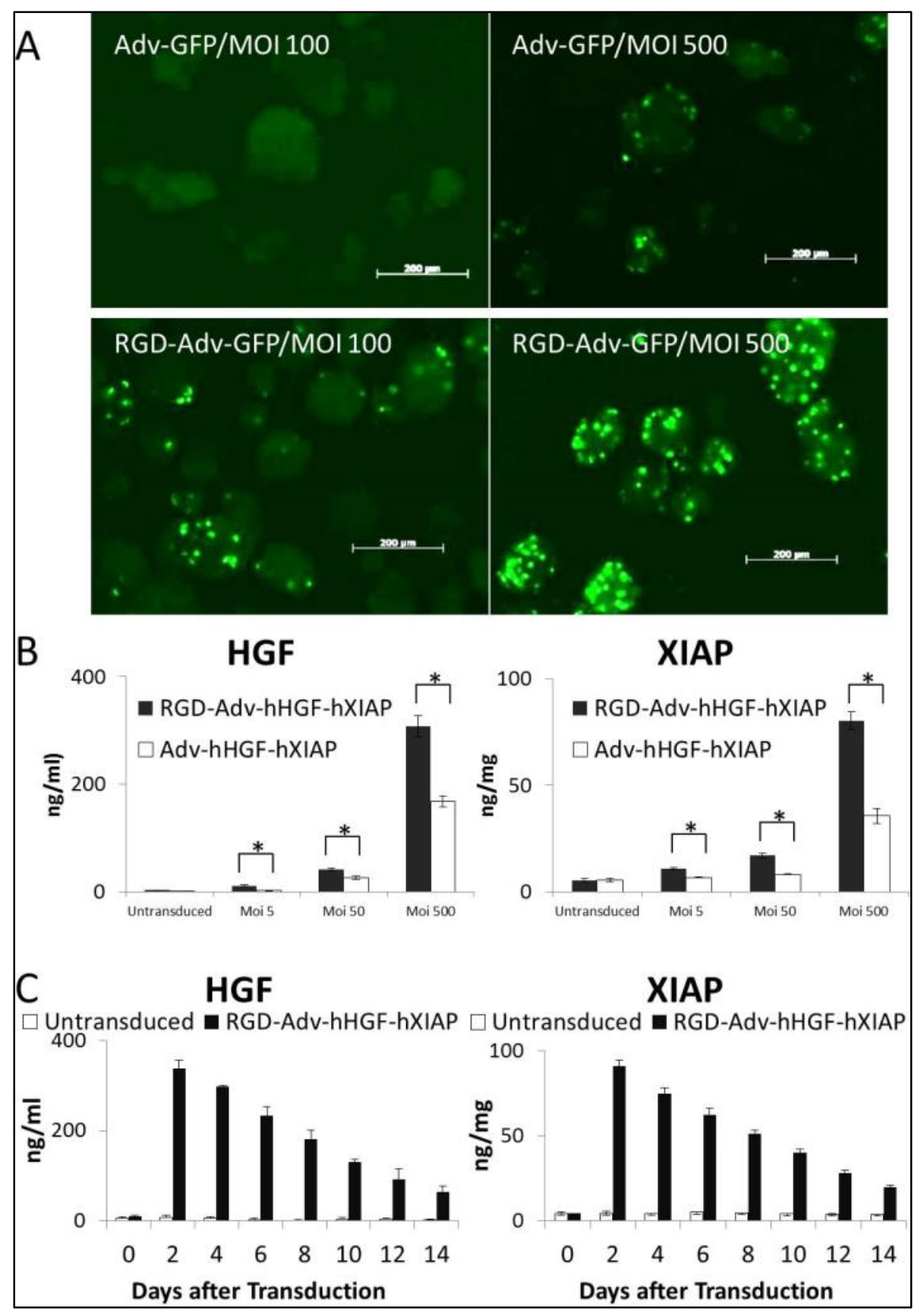

Figure 4-3. Transduction efficiency of RGD-Adv-hHGF-hXIAP into human islets A. GFP expression in human islets transduced with Adv-GFP and RGD-Adv-GFP. B. Dose dependent expression of HGF and XIAP in RGD-Adv-hHGF-hXIAP and AdvhHGF-hXIAP transduced human islets. C. Time dependent expression of HGF and XIAP in RGD-Adv-hHGF-hXIAP transduced human islets at 500 MOI. 


\section{Pro-apoptotic gene inhibited and anti-apoptotic gene elevated}

Both HGF and XIAP hold antiapoptotic effect. HGF inhibited the apoptotic pathway via activating AKT kinase while XIAP was well known for its inhibition of caspase activities $[112,113]$. Results showed that the activity of caspase 3 was significantly decreased in RGD-Adv-hHGF-hXIAP-transduced human islets over 14 days after transduction (Figure 4-4). Western blot results showed the upregulation of p-AKT at 2 days after Adv transduction. These results suggested anti-apoptotic effect of RGDAdv-hHGF-hXIAP on human islets. We also observed elevated anti-apoptotic protein BCL-2 and suppressed pro-apoptotic protein BAX, which might be a result of XIAP overexpression and degradation as suggested by the reports from Deveraux et al. and Bratton et al. [114, 115].

\section{Protection of RGD-Adv-hHGF-hXIAP on human islets}

TUNEL assay was used to determine the apoptotic cell death under cytokine treatment. We first test the anti-apoptotic effect of RGD-Adv-hHGF-hXIAP on insulinproducing INS-1E cells because it is relatively easier to stain and detect the dead cell in monolayer. Numerous apoptotic cells were observed in untransduced INS-1E cells after cytokine treatment, while much fewer apoptotic cells were observed in RGD-Adv-hHGFhXIAP-transduced INS-1E cells (Figure 4-5). Similarly, the ratio of apoptotic cells was significantly reduced in RGD-Adv-hHGF-hXIAP-transduced islets compared with untransduced islets (Figure 4-5). However, it should be addressed that since flow cytometry did not differentiate insulin-producing $\beta$ cells from other cell types in human islets, these results only suggested that the whole islet can be protected from cytokine induced cell death. Another glucose perifusion experiment is needed to determine the viability and function of insulin-producing $\beta$ cells of human islets.

Human islets were first transduced with RGD-Adv-hHGF-hXIAP and then stimulated with a cytokine cocktail of IL-1 $\beta$ and TNF- $\alpha$. Results showed that insulin secretion by human islets was significantly impaired after treated with the cytokine cocktail (Figure 4-6). However, the insulin secretion of RGD-Adv-hHGF-hXIAP transduced human islets was not significantly impaired under cytokine treatment (Figure 4-6), suggesting improved viability and function of the insulin-producing $\beta$ cells of RGDAdv-hHGF-hXIAP transduced human islet.

\section{Generation of diabetic NOD-SCID mice for human islet transplantation}

Fifteen NOD-SCID mice were used for each protocol. STZ was injected at 40 $\mathrm{mg} / \mathrm{kg}$ for 5 shots, $150 \mathrm{mg} / \mathrm{kg}$ for 1 shot, and $70 \mathrm{mg} / \mathrm{kg}$ for 2 shots. The blood glucose level was determined by a glucometer at 3 weeks after last STZ injection. A boxplot of the minimum, the 10 percentile, the median, the 90 percentile, and the maximum as draw for each protocol as shown in Figure 4-7. The box area between the 10 percentile and 90 percentile indicated the expected glucose levels for $80 \%$ of mice. The blood glucose level of $600 \mathrm{mg} / \mathrm{dL}$ was the maximum for all three protocols because it is the maximum 


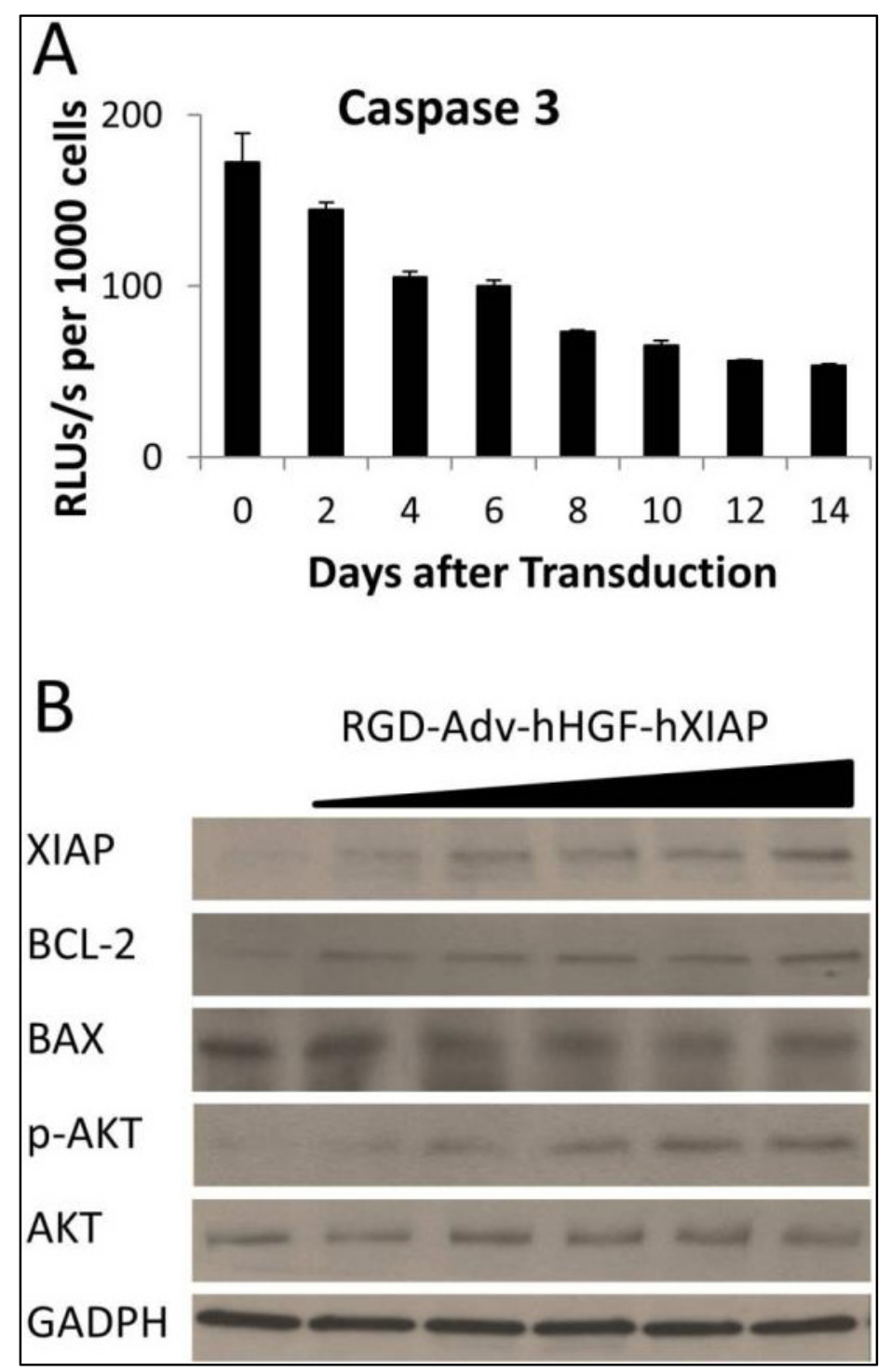

Figure 4-4. HGF and XIAP expression led to inhibition of pro-apoptotic gene and elevation of anti-apoptotic gene

A. Caspase 3 activity in RGD-Adv-hHGF-hXIAP transduced human islets was measured up to 14 days after transduction by Caspase 3 Glo kit. B. Expressions of proapoptotic marker BAX and antiapoptotic markers BCL-2, p-Akt were determined by western blot. MOI: 0, 5, 10, 50, 100, 500, left to right. 


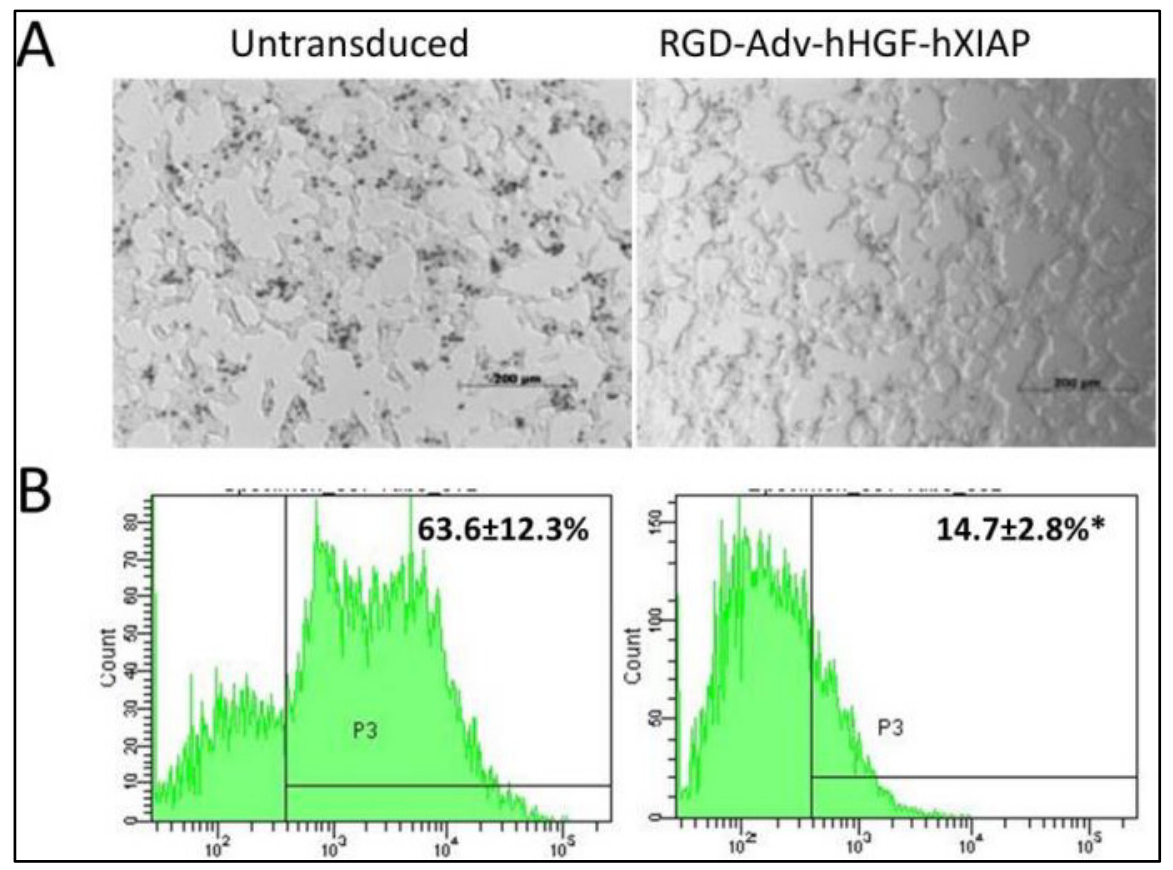

Figure 4-5. Transgene expression of HGF and XIAP protected INS-1E cells and human islets from inflammatory cytokine induced apoptotic cell death

After transduction with RGD-Adv-hHGF-hXIAP, INS-1E cells and human islets were incubated with a cytokine cocktail of IL- $\beta(5 \mathrm{ng} / \mathrm{mL})$ and TNF- $\alpha(5 \mathrm{ng} / \mathrm{mL})$ for $48 \mathrm{~h}$ and 3 days, respectively. A. Apoptotic INS-1E cells were stained dark brown using DeadEnd $^{\mathrm{TM}}$ Colorimetric TUNEL System. B. Islet was dispersed with trypsin into single cell suspension. Apoptotic cells were stained with annexin V-FITC and counted by flow cytometry. P3 indicated the percentage of apoptotic cells. All experiments were performed in triplicate and at least twice with similar results. Results are presented as mean \pm SD. ${ }^{*}, \mathrm{P}<0.05$ as determined by Student $\mathrm{t}$ test. 


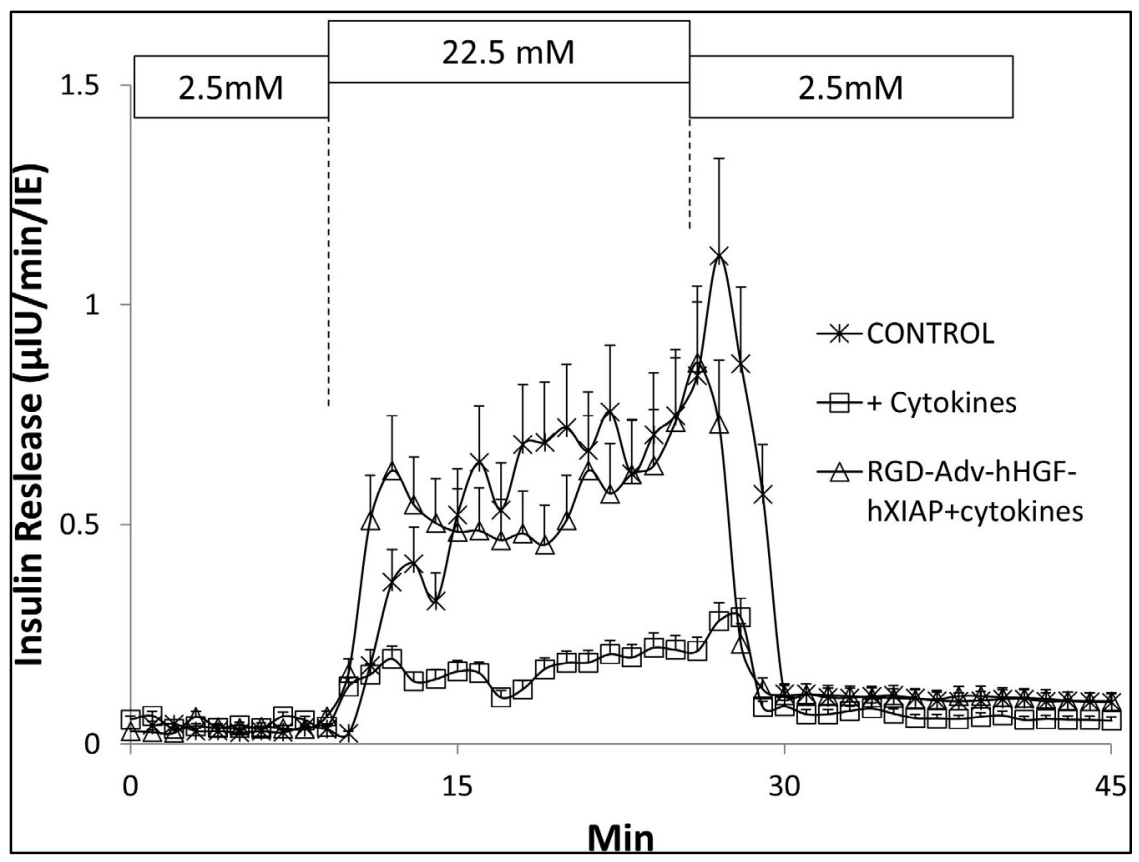

Figure 4-6. Effect of HGF and XIAP gene expression on islet function

Briefly, after transduction with RGD-Adv-hHGF-hXIAP and further stimulation with a cytokine cocktail of IL- $\beta(5 \mathrm{ng} / \mathrm{mL})$ and TNF- $\alpha(5 \mathrm{ng} / \mathrm{mL})$ for 3 days, 50 islets from each group were sequentially perifused with basal glucose $(2.5 \mathrm{mM})$ for $45 \mathrm{~min}$ and stimulated with glucose $(22.5 \mathrm{mM})$ for $15 \mathrm{~min}$ and at last perifused with basal glucose $(2.5 \mathrm{mM})$ till insulin release reverse to basal level. The flow rate was maintained at $2 \mathrm{ml} / \mathrm{min}$. Samples were collected through a fraction collector and analyzed for insulin content by ELISA. All experiments were performed in triplicate. Results are presented as Mean $\pm S D$. $n=3$. 


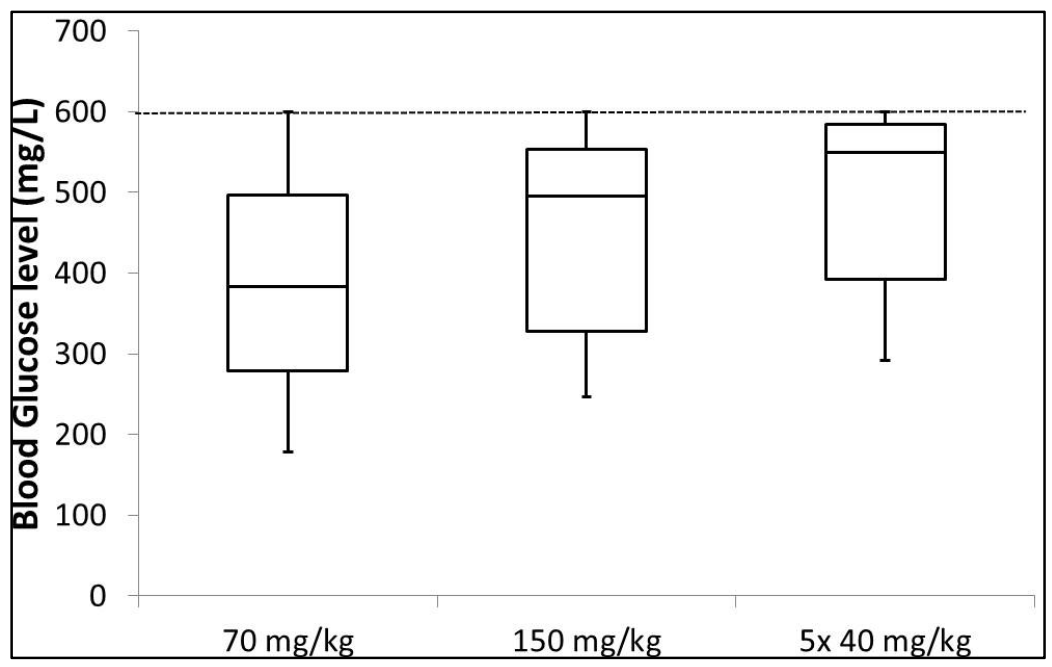

Figure 4-7. The manner and dose of STZ injection has great impact on the diabetic level of NOD-SCID mice

In the first protocol, two shots of STZ were injected intraperitoneally into NOD-SCID mice at a dose $70 \mathrm{mg} / \mathrm{kg}$ for two consecutive weeks. If the mice developed moderate level of diabetes (blood glucose 300 500 mg/dL) in the first week, the second shot can be skipped. In the second protocol, one shot of STZ was injected intraperitoneally into NOD-SCID mice at a fixed dose $150 \mathrm{mg} / \mathrm{kg}$. In the third protocol, STZ was injected intraperitoneally into NOD-SCID mice at a dose of $40 \mathrm{mg} / \mathrm{kg}$ for 5 consecutive days. The levels of blood glucose of all mice were determined by a glucometer at 3 weeks after last STZ injection. A boxplot of the minimum, the 10 percentile, the median, the 90 percentile, and the maximum was draw for each protocol. 
measurement level for the glucometer. The NOD-SCID mice receiving two shots of STZ at $70 \mathrm{mg} / \mathrm{kg}$ developed a moderate level of diabetes with $80 \%$ of them at $300 \sim 500 \mathrm{mg} / \mathrm{dL}$ and a median of $400 \mathrm{mg} / \mathrm{dL}$. The NOD-SCID mice receiving one shot of STZ at $150 \mathrm{mg} / \mathrm{kg}$ were slightly more diabetic with $80 \%$ of them at $300 \sim 550 \mathrm{mg} / \mathrm{dL}$. But the median glucose was increased to $500 \mathrm{mg} / \mathrm{dL}$. Most NOD-SCID mice receiving 5 shots of $\mathrm{STZ}$ at $40 \mathrm{mg} / \mathrm{kg}$ were over diabetic, which was consistent with our previous study that constant insulin injection was needed for glucose control besides islet transplantation.

We gathered a lot of useful information from this study. First, mice responded in a drastically different manner to STZ injection. For example, half of the mice developed moderate level of diabetes after the first shot of STZ at $70 \mathrm{mg} / \mathrm{kg}$. But the other half were not diabetic $(<200 \mathrm{mg} / \mathrm{dL})$ before the second shot. Therefore, we developed a flexible protocol in which the blood glucose of all mice could be measured in the middle of STZ injection and the non-diabetic mice would receive a second shot. Secondly, some mice developed a pre-diabetes symptom with blood glucose at $180 \sim 250 \mathrm{~m} / \mathrm{dL}$ after the first shot of STZ. These mice had a chance back to normoglycemia without any treatment. But the mice with blood glucose higher than $300 \mathrm{mg} / \mathrm{dL}$ hardly reversed normoglycemia. Therefore a blood glucose level of $300 \mathrm{mg} / \mathrm{dL}$ was used to determine whether the mouse was "truly" diabetic and ready for the following islet transplantation.

\section{RGD-Adv-hHGF-hXIAP transduction prolonged islet survival after transplantation}

Islet transplantation reversed diabetes in every subject (Figure 4-8). However, the mice transplanted with RGD-Adv-hHGF-hXIAP-transduced islets showed clearly better transplantation outcome in terms of average blood glucose level, duration of normoglycemia, and insulin independent ratio (Figure 4-8). These results demonstrated the improved viability and function of human islets genetically modified with RGD-AdvhHGF-hXIAP.

Intraperitoneal glucose tolerance test was performed 30 days after islet transplantation to determine whether ex vivo transduction of islets with RGD-AdvhHGF-hXIAP helped islet grafts engraft and respond to elevated blood glucose in real time. Prior to glucose injection, all mice displayed similar baseline blood glucose levels following overnight fasting. Blood glucose levels increased immediately following glucose $(2 \mathrm{~g} / \mathrm{kg})$ injection, peaked at $15 \mathrm{~min}$ in all groups, and decreased over time (Figure 4-9). Results showed faster and better response to the glucose boost in those mice receiving RGD-Adv-hHGF-hXIAP-transduced human islets relative to the mice receiving untransduced islets (Figure 4-9). These results demonstrated that RGD-AdvhHGF-hXIAP-transduced human islets had a better engraftment outcome and led to superior glucose control and to tolerance as compared with untransduced islets. 


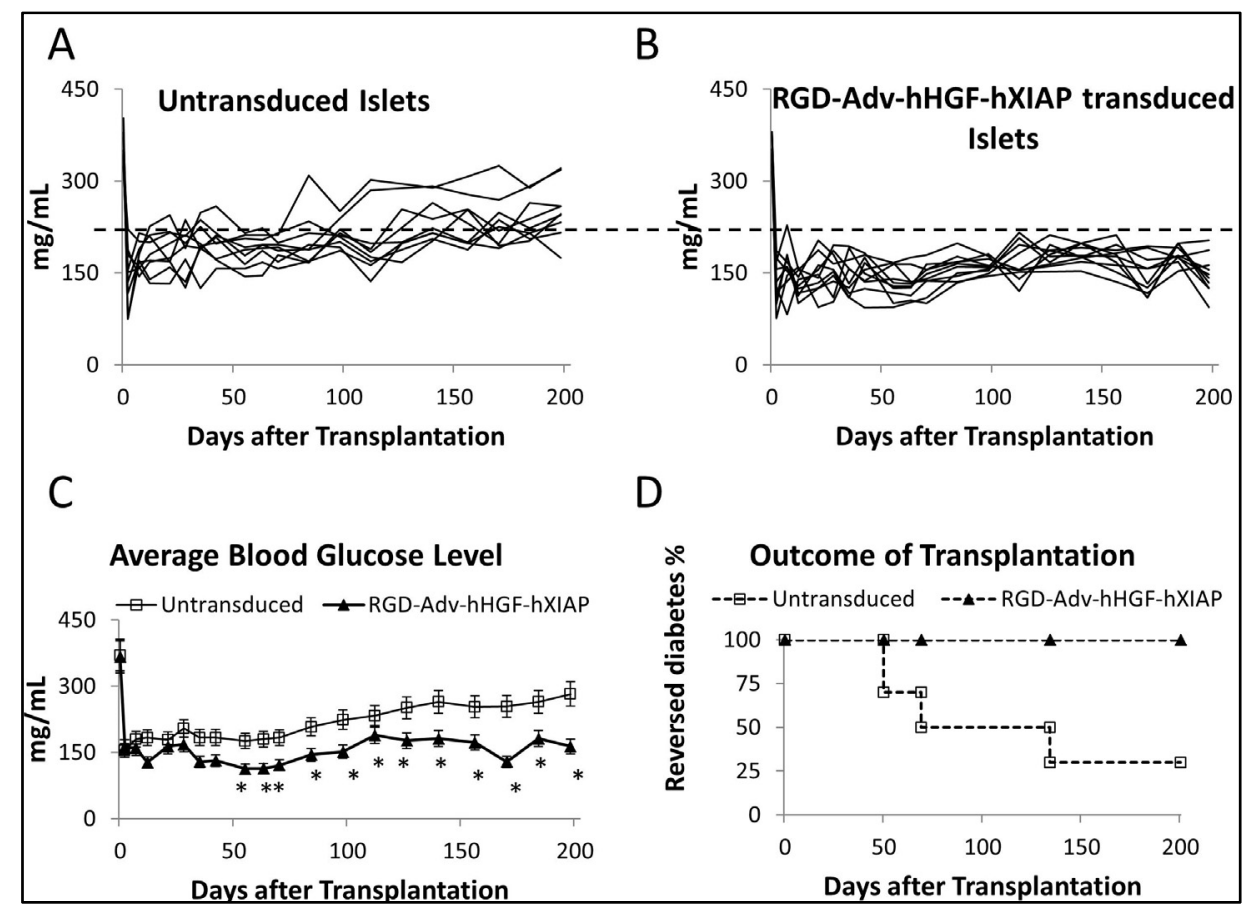

Figure 4-8. Effect of RGD-Adv-hHGF-hXIAP transduction on the outcome of islet transplantation

A-B. The blood glucose level of every single mouse after receiving 500 untransduced (A) or RGD-Adv-hHGF-hXIAP-transduced islets (B). C. The average blood glucose level of mice after islet transplantation. D. Reversed-diabetes ratio of the NOD-SCID mice after islet transplantation. Blood glucose $\leq 200 \mathrm{mg} / \mathrm{dL}$ (dashed line in a, b) was identified as reversed-diabetes. Data are presented as the mean $\pm \mathrm{SD}, \mathrm{n}=10$. ${ }^{*} \mathrm{P}<0.05$ as determined by Student $t$ test. 


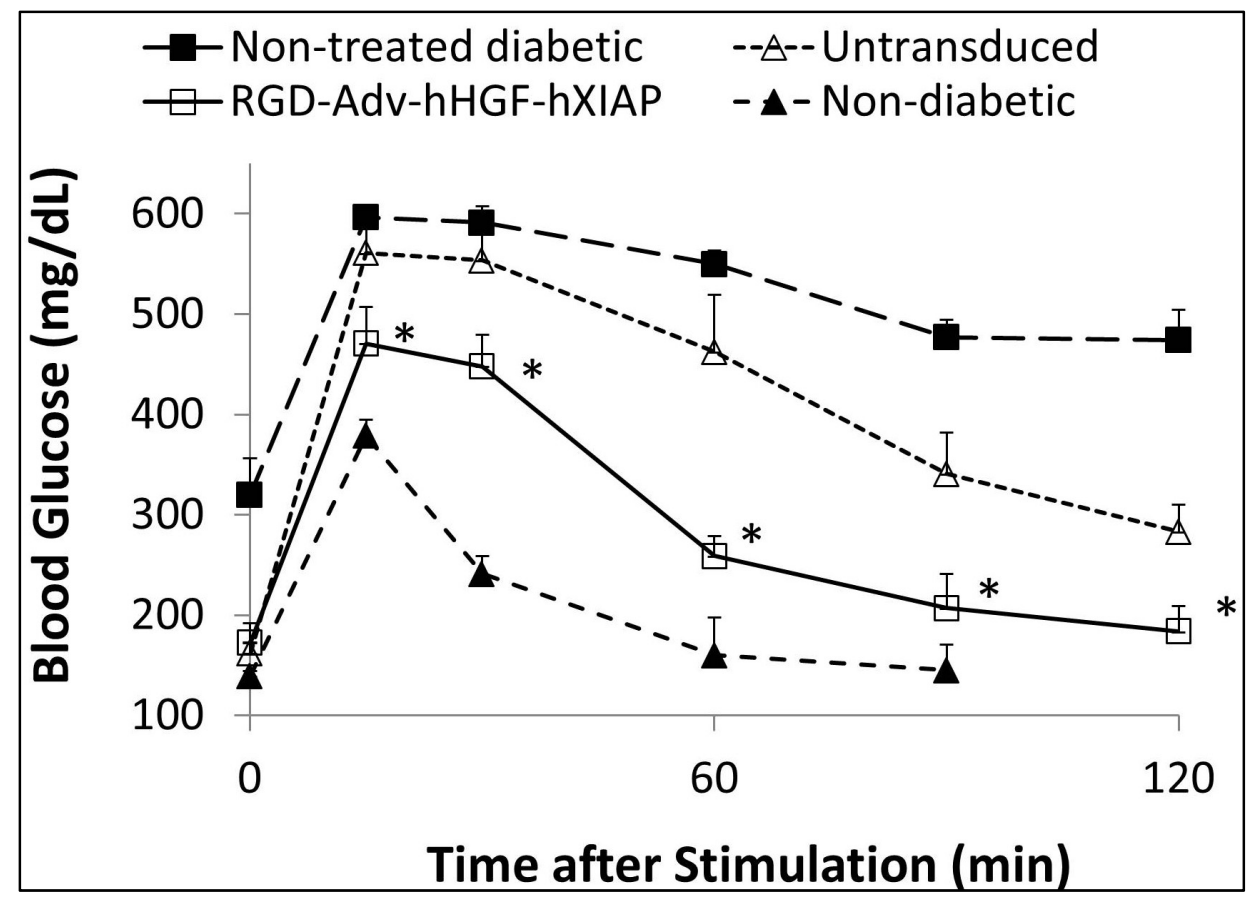

Figure 4-9. Intraperitoneal glucose tolerance test of the mice at 30 days after islet transplantation

Results from non-treated diabetic mice and non-diabetic mice were used as negative and positive controls, respectively. Data are presented as the mean $\pm \mathrm{SD}, \mathrm{n}=6$. ${ }^{*} \mathrm{P}<0.05$ as determined by Student $t$ test compared with mice receiving untransduced human islets. 


\section{RGD-Adv-hHGF-hXIAP transduction improved islet revascularization after transplantation}

As shown in Figure 4-10, immunofluorescence staining showed the successful engraftment of insulin-positive islets under the kidney capsule 30 days after islet transplantation. Untransduced islets and RGD-Adv-hHGF-hXIAP-transduced islets both showed proper engraftment and function at this time. At 200 days after transplantation, the clear insulin staining in the kidney bearing RGD-Adv-hHGF-hXIAP transduced islets indicated morphological integrity and appropriate function of transplanted islets (Figure 4-11). In addition, the potent staining of vWF in proximity to these islets indicated the functional revascularization of islet grafts under the kidney capsule. However, in the kidney bearing untransduced islets, only scarce vWF staining was seen and weak insulin staining indicated poor morphology and function of transplanted islets.

\section{Discussion}

PNF is the major reason leading to islet loss after transplantation except immune rejection. PNF is mostly caused by the inflammatory cytokines and poor revascularization. Dinarello et al. summarized that IL-1 and TNF are the two most important effectors in autoimmune diseases [27]. Donath et al. concluded that antiinflammatory therapeutic approaches to block $\beta$-cell apoptosis could be an important strategy in treating type 1 and 2 diabetes [28]. We previously reported that IL-1Ra expression in human islets effectively blocked the IL-1 $\beta$-mediated apoptotic islet death [8, 29], but it did not stop the apoptosis caused by other inflammatory cytokines such as TNF $\alpha$ and IFN $\gamma$. We also reported that XIAP expression in human islets repressed apoptotic islet death by inhibiting caspase activation [30], but its effect is limited because of low transduction efficiency. In the current study, we genetically engineered Adv with an RGD peptide to improve transduction efficiency and co-expressed HGF and XIAP, both of which hold anti-apoptotic effect but through different pathways [21, 31]. Our results demonstrated that the levels of HGF and XIAP were significantly upregulated and gradually decreased 14 days after Adv transduction (Figure 4-3C), which accords with the transient property of Adv-mediated gene expression. Consequently, a high HGF level activated the PI3K-Akt pathway and a high XIAP level suppressed caspase 3 activity in the first 14 days after Adv transduction (Figure 4-4). In an inflammatory condition mimicked by a cytokine cocktail of IL- $1 \beta$ and TNF $\alpha$, TUNEL assay and islet perifusion study indicated that overexpression of HGF and XIAP gene prior to cytokine treatment effectively protected islet viability and function from apoptotic cell death (Figures 4-5, 4-6). The in vivo viability and function were further confirmed by immune fluorescence analysis (Figure 4-10).

Revascularization is another crucial factor for successful islet transplantation. Islets are a cluster of heterogeneous cell types with extensive intra-islet vessels. These vessels get disrupted during islet isolation, leading to the collapse of vasculature, the accumulation of endothelial fragments, and the compromised perfusion in the core of the 


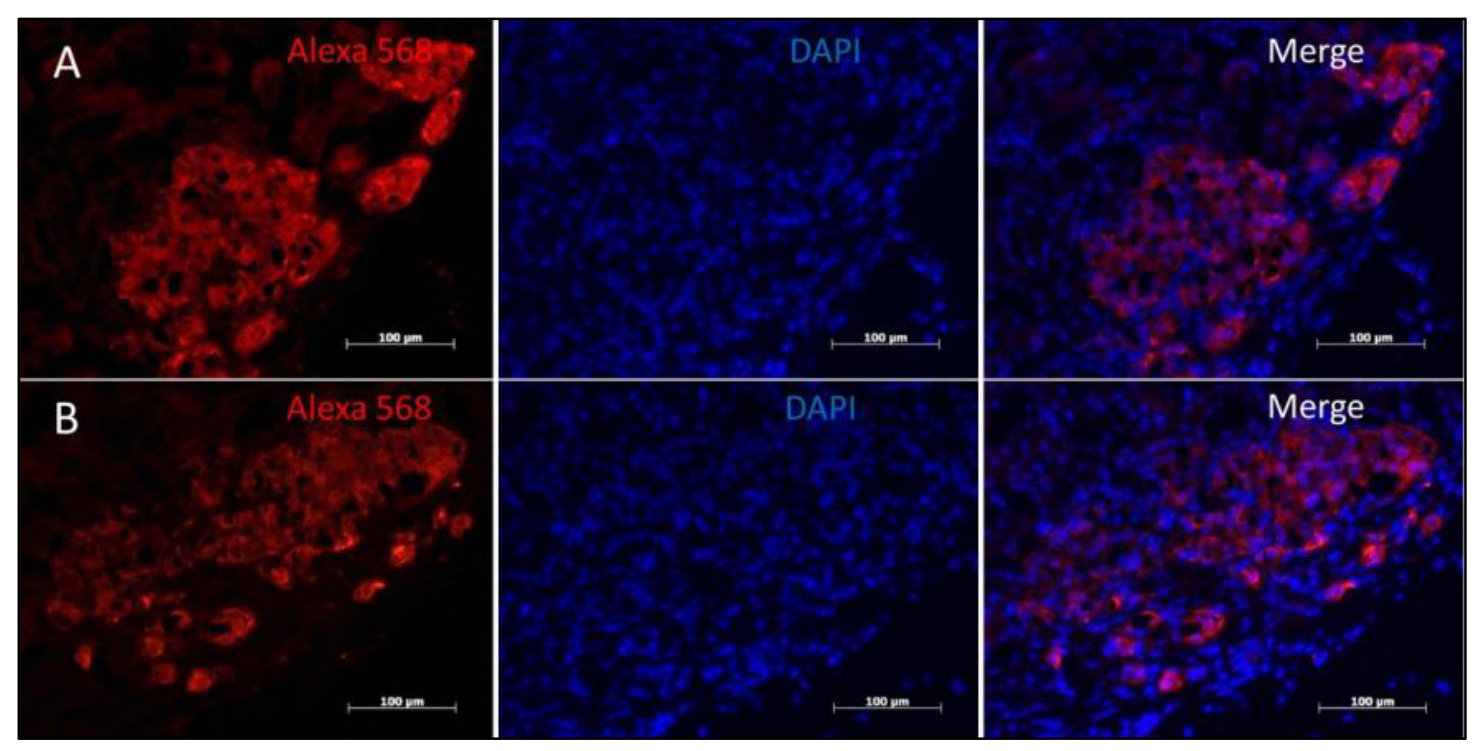

Figure 4-10. Immunofluorescence staining of the kidney section bearing RGD-AdvhHGF-hXIAP-transduced human islets at 30 days after islet transplantation A. Untransduced human islets. B. RGD-Adv-hHGF-hXIAP-transduced human islets. Human insulin was stained in red. Slides were back-stained with DAPI. Scale bar represents $100 \mu \mathrm{m}$. 


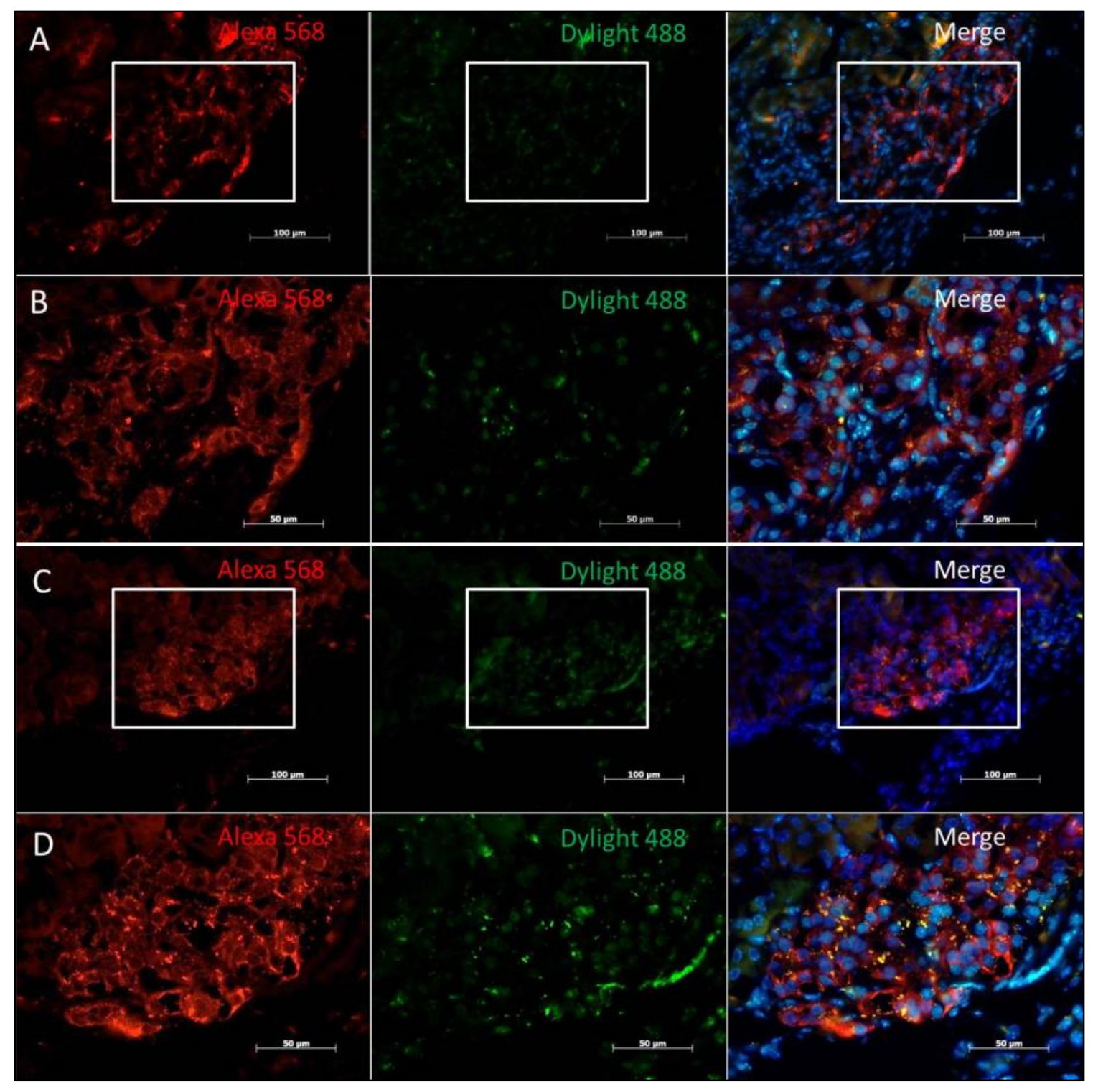

Figure 4-11. Immunofluorescence staining of the kidney section bearing RGDAdv-hHGF-hXIAP-transduced human islets at $\mathbf{2 0 0}$ days after islet transplantation Insulin was stained in red (first column) and vWF factor was stained in green (second column). A-B. Kidney section bearing untransduced human islets under 10x and 40x magnification. C-D. Kidney section bearing RGD-Adv-hHGF-hXIAP-transduced human islets human islets under 10x and 40x magnification. 
islets [32]. Therefore, extensive and functional revascularization is required to promote the survival of islet grafts posttransplantation [33]. VEGF is a popular angiogenic factor, but its effect is so potent that caution should be exercised to avoid aberrant angiogenesis [34]. Recently, HGF has been seen as a more favorable growth factor because it can prevent apoptotic islet death and can promote the proliferation of pancreatic $\beta$-cells [12]. In this study, we demonstrated that HGF expression of human islets can be upregulated over 14 days after Adv transduction (Figure 4-3) Consequently, extensive revascularization was observed in the mice transplanted with RGD-Adv-hHGF-hXIAPtransduced human islets in the immune fluorescence study (Figure 4-11).

Islet destruction following transplantation can be greatly reduced by gene therapy [35]. Since islet is a compact cluster of about 1000 non-dividing cells, it is difficult to transfect intact islets by the available non-viral approaches, such as cationic liposomes and polymer-based systems, which are also toxic at high doses [36]. In contrast, replication deficient (E1, or E3 deleted) Adv vectors are known to efficiently transduce islets. In addition, adenoviral vectors can be produced in high titers and there is no risk of insertional mutagenesis as they do not integrate into host genome. However, Adv transduction of human islets leads to increasing immune responses, because some viral genomes co-express with therapeutic genes and elicit increased immune response and allograft rejection $[37,38]$. This problem may be exaggerated when a relatively higher MOI is required to achieve optimal transduction efficiency on solid organs like human islets $[13,14]$.

We incorporated an RGD peptide sequence into the Adv fiber knob to interact with $\alpha v \beta$ integrins to facilitate Adv transduction into human islets [39]. Our results indicated that the transduction efficiency on human islets could be significantly improved using RGD-modified Adv (Figure 4-1). Transduction efficiency could be further increased when MOI was increased to 1000 and 5000 (data not shown). However, we decided not to transduce islets with an MOI higher than 500 to avoid any toxic side effects. The insulin perifusion study demonstrated that there was no decrease in insulin production from RGD-Adv-hHGF-hXIAP-transduced islets (Figure 4-6). RGD modification of Adv may be of significant importance to the development of new viral vectors, which can be genetically redirected to specialized cells as gene delivery vehicles. A similar RGD sequence placed in the virus hexon coat protein increased gene transfer to cells that were normally refractory to Adv transduction [40]. Similarly, adding a cationic polylysine sequence (pK7) to the fiber knob $\mathrm{COOH}$ terminus, which facilitates viral binding to negatively charged cell surface molecules such as heparan sulfates, increased transduction to macrophages, endothelial cells, smooth muscle cells, fibroblasts, and T cells $[41,42]$. In this study, we also evaluated a bipartite Adv vector for multiple gene delivery to human islets for promoting revascularization and inhibiting inflammatory cytokine-mediated destruction. Because transfection with the bipartite plasmid encoding hHGF and hXIAP (dI324-hHGF-hXIAP-RGD, Figure 4-1) produced very little hHGF or hXIAP (data not shown), viral vector seems to be the only choice for gene therapy on human islets. Bipartite Adv vectors are usually constructed with one therapeutic gene and another marker gene, especially GFP [43, 44]. Using a bipartite vector not only simplifies the amplification and purification process of Adv vectors but also decreases the use of 
total Adv backbone, compared to the use of two single Adv vectors. This procedure is expected to minimize the immunogenicity and toxicity of Adv vectors.

Islet isolation and preparation is another state-of-the-art in clinical islet transplantation. Unlike other species from which islets can be isolated with little contamination, human islet preparations are usually composed of a majority $(50 \sim 60 \%)$ of $\beta$ cells and many other cell types including duct cells, acinar cells, $\alpha$ cells, and lymph node cells $[45,46]$. Regardless of their origin, all cells within an islet preparation may contribute to the immune response induced after transplantation. Therefore, purity and viability of islets used for transplantation have a significant impact on the outcome of islet transplantation. Dithizone staining is carried out to discriminate between endocrine and non-endocrine cells to increase the purity of the islet preparation to $70-95 \%[46,47]$. Using hand-picked islets helped to further improve islet purity by discarding other tissues such as acinar cells and lymph nodes [48]. However, further purification of the islet preparations remains a challenge because of a remarkable loss of insulin-producing cells.

To summarize, our study demonstrated that RGD modification is a useful tool to improve the transduction efficiency of Adv. Ex vivo transduction of islets with RGDAdv-hHGF-hXIAP decreased apoptotic islet death and improved islet revascularization and eventually improved the outcome of human islet transplantation. 


\section{CHAPTER 5. BMSC AS GENE DELIVERY VEHICLE TO EXPRESS HGF AND IL-1RA TO IMPROVE THE OUTCOME OF HUMAN ISLET TRANSPLANTATION*}

\section{Introduction}

Traditional gene therapy directly transfers genetic materials into human islet using viral or nonviral vectors to generate genetically modified human islets. This strategy is promising because the resistance of islet to a specific challenge can be selectively enhanced by overexpression of a specific gene but holds some weakness because 1) human islets will be subjected to gene transfer for at least 1 day before transplantation causing inevitable PNF in the transduction process and 2) the co-expression of transgenes with viral genes could increase the immunogenicity of transduced human islets and make them easily recognized and cleared by the host immune system.

MSC are adult progenitor cells with differentiation potential and self-renewal capacity. MSCs have great promise in repairing injured tissues including pancreatic islets [42]. MSCs also hold hypoimmunogenic property [55], since they are in lack of major histocompatibility complex II (MHC-II) and co-stimulatory molecules, such as CD14, CD86, CD40L and CD95L. MSCs suppress inflammation, either by receptor-mediated signal-transduction, probably through MMP2/MMP9-CD25 signal pathway [60], or the secretion of immunosuppressive factors such as TGF- $\beta 1$, interleukin 10 (IL-10) and PGE2. Therefore, MSCs are unlikely to cause inflammation at the transplant site. These characteristics make MSCs attractive vehicles for virus-based gene therapy.

BMSC was selected for several reasons. First, BMSC can be readily isolated from bone marrow and many other tissues. Secondly, BMSC is an adult stem cell with limited expansion and differentiation potential. Therefore the tumorigenicity of BMSC was low. Thirdly, BMSC was accessible to a wide spectrum of genetic modifications, which were not reported to affect the stem-cell-like properties of BMSC. Fourthly, BMSC actively secreted multiple growth factors as a trophic mediator which can help the engraftment of human islets after transplantation. We hypothesized that BMSC genetically modified to express soluble factors can be a competent strategy to help human islet transplantation without direct transduction of human islets (Figure 5-1). However, it should be noted that BMSCs alone, through systemic administration or local transplantation, were less competent to prevent the islet loss immediately after transplantation, which accounts for more than $70 \%$ of islet loss in the first week after transplantation $[95,96]$. This phenomenon probably explains the inadequate control of the blood glucose level in the early stage $(<3$ weeks) after transplantation in most islet/MSC co-transplantation studies $[54,116]$.

\footnotetext{
* Reprinted with permission from Springer. Wu, H, Lu, W, and Mahato, RI (2011). Mesenchymal stem cells as a gene delivery vehicle for successful islet transplantation. Pharmaceutical Research 28: 2098-2109.
} 


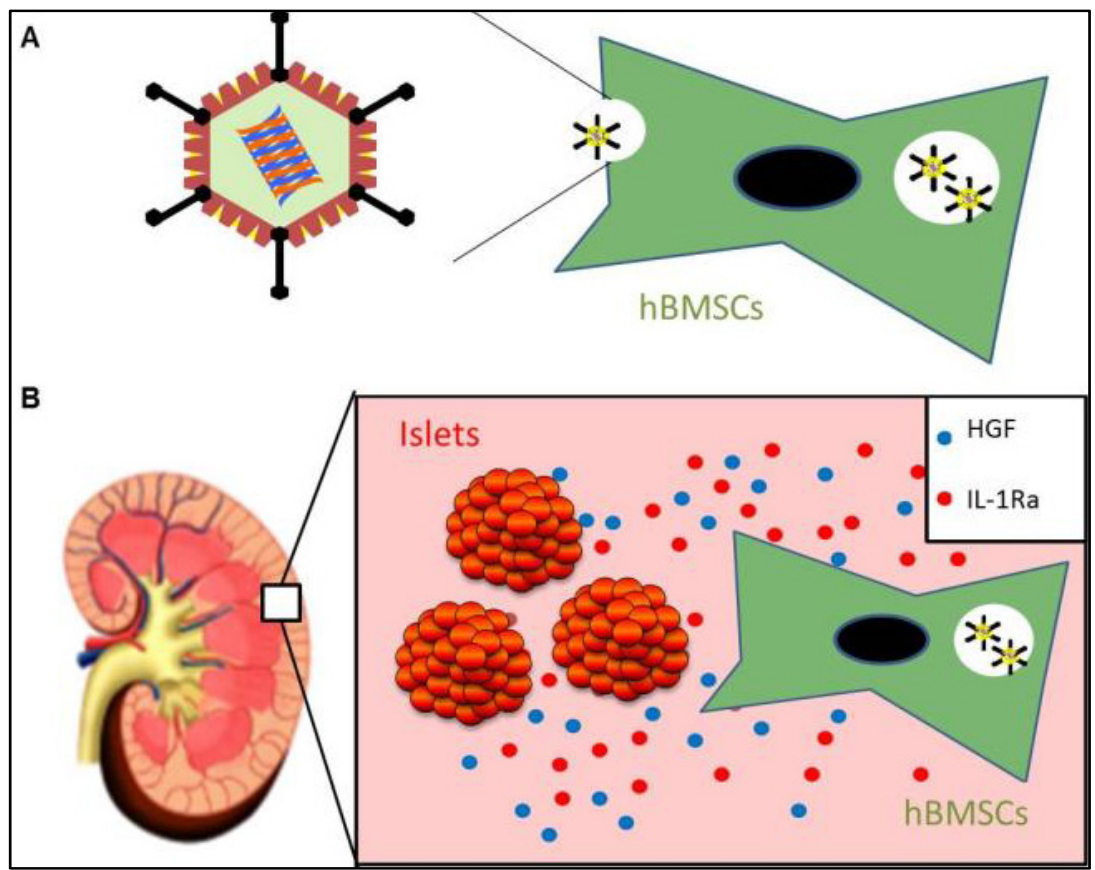

Figure 5-1. A schematic demonstration of Adv-hHGF-hIL-1Ra-transduced BMSCs co-transplantation with human islets

A. BMSCs were transduced with Adv-hHGF-hIL-1Ra prior to islet transplantation. B. After co-transplantation with human islets under the kidney capsule of diabetic NODSCID mice, BMSCs expressed HGF and IL-1Ra into the surrounding microenvironment to support islet viability and function. 
Because traditional gene transfer into human islets is less efficient and time consuming and BMSC mediated cell therapy is less competent to prevent the acute graft loss, we planned to utilize BMSC mediated gene therapy in this study. We hypothesized that adenovirus mediated gene therapy can prevent the acute graft loss while the BMSC can support the islet function for long term. We selected genes encoding HGF and IL$1 \mathrm{Ra}$, both of which were soluble factors, as the therapeutic genes in this study. The antiapoptotic effect of IL-1Ra expression may not be able to compete with XIAP because ILRa blocked the islet apoptosis caused by death receptor pathway but XIAP blocked the islet apoptosis in a more ubiquitous manner (Figure 3-1). But we avoided the toxicity associated with direct transduction and the viral expression in human islets. Moreover the gene transfer efficiency on BMSC which can be seeded in a tissue culture flask is significantly higher than human islets. BMSC can also be transduced and expanded before we received islet preparations, therefore we can save one day of gene transfer into human islets and it may significantly prevent the loss of islet due to PNF. Other benefit of this study is that BMSC was known to maintain its stem cell properties after transduction. Therefore when the transgene expression mediated by adenovirus diminished in 2 weeks, the growth factors produced by BMSC, though in a lesser extent, may help islet survival for a long duration. We were trying to test these hypotheses in the following study.

In this study, we evaluated the capability of primary BMSCs as gene delivery vehicles to protect the graft viability of human islets in a STZ induced NOD-SCID mice model. Adv-hHGF-hIL-1Ra-transduced BMSCs served as gene delivery vehicles to express HGF to promote islet revascularization, and IL-1Ra to reduce acute islet loss from inflammatory cytokines. We also determined the capability of BMSCs as nursing cells to prevent long-term islet graft rejection.

\section{Materials and Methods}

\section{Materials}

The replication-deficient $(\Delta \mathrm{E} 1 / \Delta \mathrm{E} 3)$ Adv-hHGF-hIL-1Ra containing a CMV promoter, hHGF cDNA, and rabbit $\beta$-globin poly $A$ in the E-1 region, and a CMV promoter, hIL-1Ra cDNA, and rabbit $\beta$-globin poly $\mathrm{A}$ in the E-3 region was constructed amplified and the titer measured in our lab as previously described (Figure 5-2) [8]. The control virus encoding green fluorescent protein (Adv-GFP) was constructed and kept in our lab. Primary BMSCs, HyClone Advanced Stem Cell Medium and Medium Supplement were purchased from Thermo Fisher Scientific (Waltham, MA), passaged, and frozen in our lab. Human islets were received from Integrated Islet Distribution Program (Duarte, CA). CMRL-1066 medium for islet culture and 6-diamidino-2phenylindole (DAPI) were purchased from Sigma Aldrich (St. Louis, MO). FBS was purchased from MediaTech Cellgro. (Herndon, VA). PBS was purchased from GIBCOBRL (Gaithersburg, MD). Human HGF ELISA kits, IL-1Ra ELISA kits, IL-10 ELISA kits, VEGF ELISA kits, Stem cell factor (SCF) ELISA kits and recombinant IL-1 $\beta$ were purchased from R\&D Systems (Minneapolis, MN). Human insulin and c-peptide ELISA 


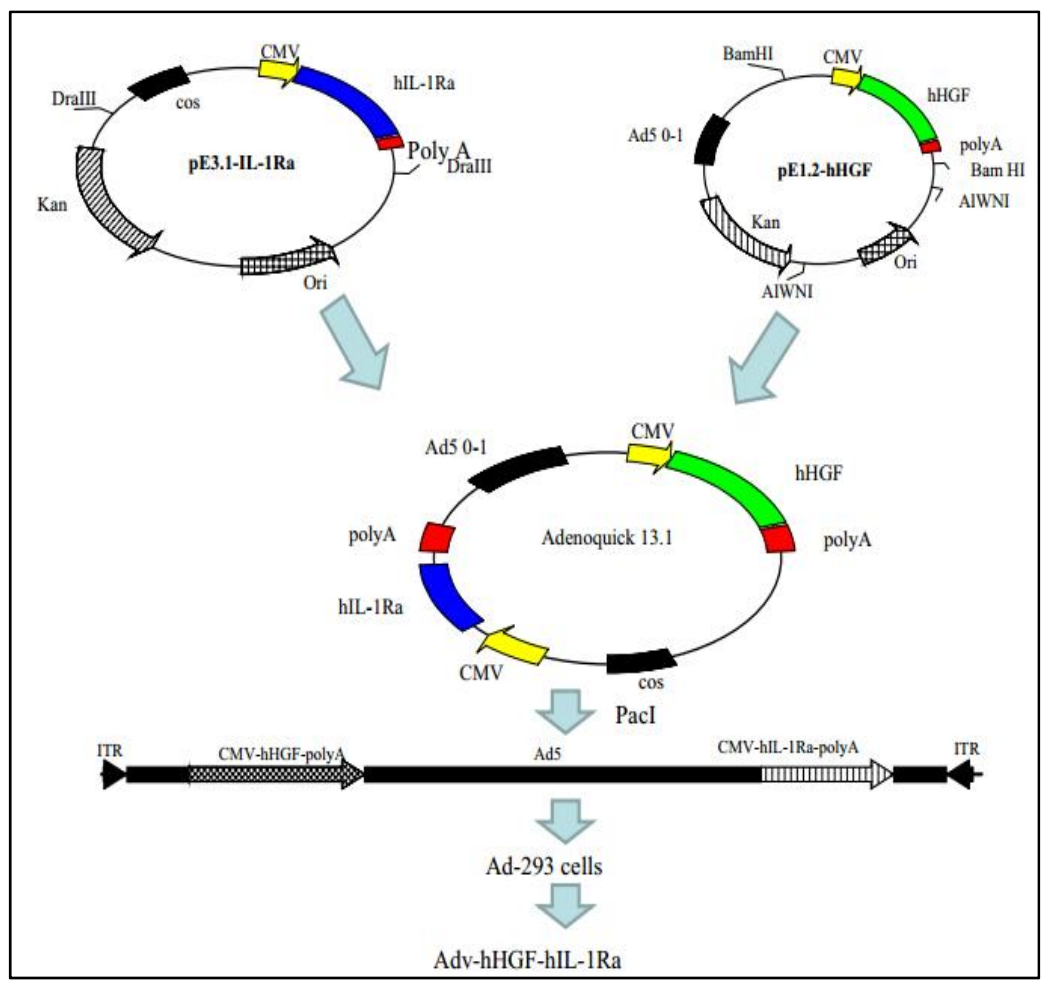

Figure 5-2. Construction of bipartite Adv-hHGF-hIL-1Ra using AdenoQuick cloning system

Shuttle plasmids pE 3.1 which contains IL-1Ra gene and pE 1.2 which contains HGF gene were cloned into AdenoQuick plasmid 13.1 to generate a cosmid containing genes of interest and the entire sequence of recombinant adenovirus by homologous recombination. After transfection into 293 cells, recombinant adenovirus Adv-hHGFhIL-1Ra was produced. 
kits were purchased from Alpco Diagnostics (Windham, NH). The primary antibodies for hHGF, hIL-1Ra, insulin, vWF, and the Dylight 488-conjugated secondary antibody were purchased from Abcam (Cambridge, MA). The Alexa Fluor 568-conjugated secondary antibody and $0.25 \%$ trypsin was purchased from Invitrogen (Carlsbad, CA). 8-well LabTek Chamber Slides were purchased from Nalge Nunc. (Rochester, NY). Ultrasensitive One Touch glucose test strips and One Touch Ultra glucometer were purchased from LifeScan (Milpitas, CA). Tissue-Tek O.C.T. compounds were purchased from Sakura Finetek (Torrance, CA).

\section{Adv transduction of BMSCs}

Primary BMSCs were seeded at a density of $5 \times 105$ cells per well with $2 \mathrm{ml}$ HyClone Advanced Stem Cell Medium in a 6-well plate and allowed $24 \mathrm{~h}$ for adherence. Prior to transduction, morphology of BMSCs was checked under a bright field microscope. To determine the optimal MOI for Adv transduction, primary BMSCs were transduced with Adv-GFP at $60 \mathrm{MOI}$ and $120 \mathrm{MOI}$ for $3 \mathrm{~h}$ followed by washing three times. The GFP expression was determined under a fluorescent microscope at 2 days after transduction. Primary BMSCs were then transduced with Adv-hHGF-hIL-1Ra at $120 \mathrm{MOI}$ for $3 \mathrm{~h}$ followed by washing three times. The expressions of HGF, IL-1Ra, IL10, VEGF, and SCF were measured by ELISA at $0,2,4,6,8,10,12,14$ days after transduction according to the manufacturer's instructions. The protein concentration was normalized using the number of cells in each well.

\section{Islet viability study}

After transduction with Adv-hHGF-hIL-1Ra at 120 MOI for $3 \mathrm{~h}, 5 \times 105$ primary BMSCs were digested with $0.25 \%$ trypsin and co-culture with 500 IEs in a $10 \mathrm{~cm}$ dish of CMRL-1066 medium with 10\% FBS for 2 days. Then, the islet/BMSC co-culture was stimulated with $5 \mu \mathrm{g} / \mathrm{ml} \mathrm{IL-} 1 \beta$ for 4 days and stained with $5 \mu \mathrm{g} / \mathrm{ml}$ calcein-AM and 2 $\mu \mathrm{g} / \mathrm{ml}$ propidium iodide for $30 \mathrm{~min}$. Cell viability was monitored under a fluorescent microscope. Viability of human islets was also determined by staining with dithizone, a zinc chelating agent. Briefly, at 4 weeks after cultured alone or co-cultured with AdvhHGF-hIL-1Ra-transduced primary BMSCs, human islets were stained with dithizone as described previously [12]. The insulin production of human islets was quantified by the static insulin release method. Briefly, five hundred IEs were cultured alone or with $5 \times$ 105 untransduced primary BMSCs or with $5 \times 105$ Adv-hHGF-IL-1Ra-transduced primary BMSCs (120 MOI) in a 10-cm dish of CMRL-1066 medium with $10 \%$ FBS for 10 days. At day $10,5 \mu \mathrm{g} / \mathrm{ml}$ IL-1 $\beta$ was added to the medium and the islet $/ \mathrm{BMSC}$ coculture was incubated with IL-1 $\beta$ for 4 days. Insulin release from islets was measured at day 0,10 and 14 by sequentially stimulated islets with the media containing $2.5 \mathrm{mM}$ (basal) and $22 \mathrm{mM}$ glucose (stimulated) at $37^{\circ} \mathrm{C}$ for $1 \mathrm{~h}$. 


\section{Islet transplantation}

Animal experiments were performed following NIH

(http://grants1.nih.gov/grants/olaw/references/phspol.htm) and institutional animal care and use guidelines using an approved protocol. To induce diabetes, STZ (70 mg/kg) was administered to NOD-SCID mice by intraperitoneal injection for 2 consecutive days. Animals were considered to be diabetic after two consecutive measurements of blood glucose $\geq 400 \mathrm{mg} / \mathrm{dl}$ using a glucometer. Primary BMSCs were transduced with AdvhHGF-hIL-1Ra at 120 MOI and labeled with Qdot 565 prior to transplantation according to the manufacturer's instructions. Then islets were transplanted into the kidney capsule of diabetic NOD-SCID mice alone or with Adv-hHGF-hIL-1Ra-transduced primary BMSCs at constant ratio (islet: $\mathrm{BMSC}=1: 100$ ). The nonfasted glucose levels and body weights of all the mice were measured from the snipped tail of each animal up to 15 weeks after transplantation. Then the mice were anesthetized to collect blood to measure serum insulin and c-peptide levels by ELISA. The graft-bearing kidneys were then removed from some animals to confirm the function of islet grafts by the return of blood glucose levels to $\geq 400 \mathrm{mg} / \mathrm{dL}$ for 2 consecutive days.

\section{Intraperitoneal glucose tolerance test}

Two weeks after islet transplantation, glucose tolerance was analyzed in overnight fasted mice as described by Garcia-Ocana et al [9]. Briefly, the mice were subjected to intraperitoneal injection of glucose at $2 \mathrm{~g} / \mathrm{kg}$ of body weight. Blood samples were obtained from the snipped tail at 15, 30, 60, 90, 120, and 180 min after injection and analyzed for glucose levels using a glucometer.

\section{Immunofluorescence staining}

For Adv-hHGF-hIL-1Ra-transduced BMSCs, primary BMSCs were seeded at the density of $5 \times 104$ each well into an 8 -well chamber slide. After $24 \mathrm{~h}$, these cells were transduced with Adv-hHGF-hIL-1Ra at 120 MOI for $3 \mathrm{~h}$ followed by washing three times and culturing an additional $48 \mathrm{~h}$. The slides were then detached from the media chamber and fixed with ice-cold methanol. To detect HGF expression, the slides were stained with rabbit anti-HGF primary antibody $(1: 200)$ at $4{ }^{\circ} \mathrm{C}$ overnight and Alexa Fluor 568-conjugated goat anti-rabbit secondary antibody (1:500) at room temperature for $1 \mathrm{~h}$. To detect IL-1Ra expression, slides were stained with rabbit anti-IL-1Ra primary antibody $(1: 200)$ at $4{ }^{\circ} \mathrm{C}$ overnight and Dylight 488-conjugated goat anti-rabbit secondary antibody (1:500) at room temperature for $1 \mathrm{~h}$. Slides were counter-stained with DAPI.

For the kidney section, 1000 IEs were mixed with Adv-hHGF-hIL-1Ratransduced BMSCs and co-transplanted under the kidney capsule of STZ-induced diabetic NOD-SCID mice. Mice receiving islets alone and islets co-transplanted with primary BMSCs were sacrificed 4 weeks after islet transplantation. Mice receiving islets co-transplanted with Adv-hHGF-hIL-1ra transduced BMSCs were sacrificed at 4 weeks 
and 15 weeks after islet transplantation. Kidneys bearing islets were isolated, washed, fixed in 4\% paraformaldehyde overnight, and embedded in optimal cutting temperature compound. Frozen sections of $5 \mu \mathrm{m}$ thickness were cut. To detect insulin-positive human islets, the slides were stained with guinea pig anti-insulin primary antibody $(1: 200)$ at 4 ${ }^{\circ} \mathrm{C}$ overnight and Alexa Fluor 568 conjugated goat anti-guinea-pig secondary antibody (1:500) at room temperature for $1 \mathrm{~h}$. To detect revascularization, the slides were stained with rabbit anti-vWF primary antibody (1:500) at $4{ }^{\circ} \mathrm{C}$ overnight and Dylight 488 conjugated goat anti-rabbit secondary antibody (1:500) at room temperature for $1 \mathrm{~h}$. Slides were counter-stained with DAPI. Images were analyzed using the Measure RGB function of ImageJ to quantify the relative intensity of protein expression.

\section{Statistical analysis}

Statistical significance of the difference between the two groups was determined by unpaired t-test and between several groups by one-way ANOVA.

\section{Results}

\section{Characterization of Adv-hHGF-hIL-Ra-transduced BMSCs}

As shown in Figure 5-3, primary BMSCs exhibit a spindle-shaped fibroblastic morphology after ex vivo expansion. The optimal MOI was determined by transduction of BMSCs with Adv-GFP at $60 \mathrm{MOI}$ and $120 \mathrm{MOI}$. Results showed that the Adv transduction efficiency into primary BMSCs is $>30 \%$ at $60 \mathrm{MOI}$ and $>80 \%$ at $120 \mathrm{MOI}$. Then 120 MOI was selected for optimal transduction of Adv-hHGF-hIL-1Ra. Results showed that primary BMSCs transduced with Adv-hHGF-hIL-Ra at 120 MOI produced significantly elevated levels of hHGF and hIL-1Ra since two days after transduction. The expressions of HGF and IL-1Ra by Adv-hHGF-hIL-1Ra transduced primary BMSCs decreased with time but were still significantly higher than the expressions by untransduced BMSCs at 14 days after transduction. The exogenous expressions of HGF and IL-1Ra by Adv-hHGF-hIL-1Ra transduced primary BMSCs were validated by immunofluorescence staining. Primary BMSCs produced IL-10 in picogram level and VEGF in nanogram level and the native expressions of IL-10 and VEGF were not changed by Adv transduction (data not shown). As in previous reports $[117,118]$, we did not detect expression of SCF from primary BMSCs (data not shown). In summary, these results indicated that primary BMSCs simultaneously expresses the target exogenous gene after transduction by Adv-hHGF-hIL-1Ra without losing their native characteristics.

\section{Protection of human islets by Adv-hHGF-hIL-Ra-transduced BMSCs}

Primary BMSCs participated extensively in organ repair [119]. HGF and IL-1Ra were widely reported to hold anti-apoptotic ability $[120,121]$. We tested the protection of 

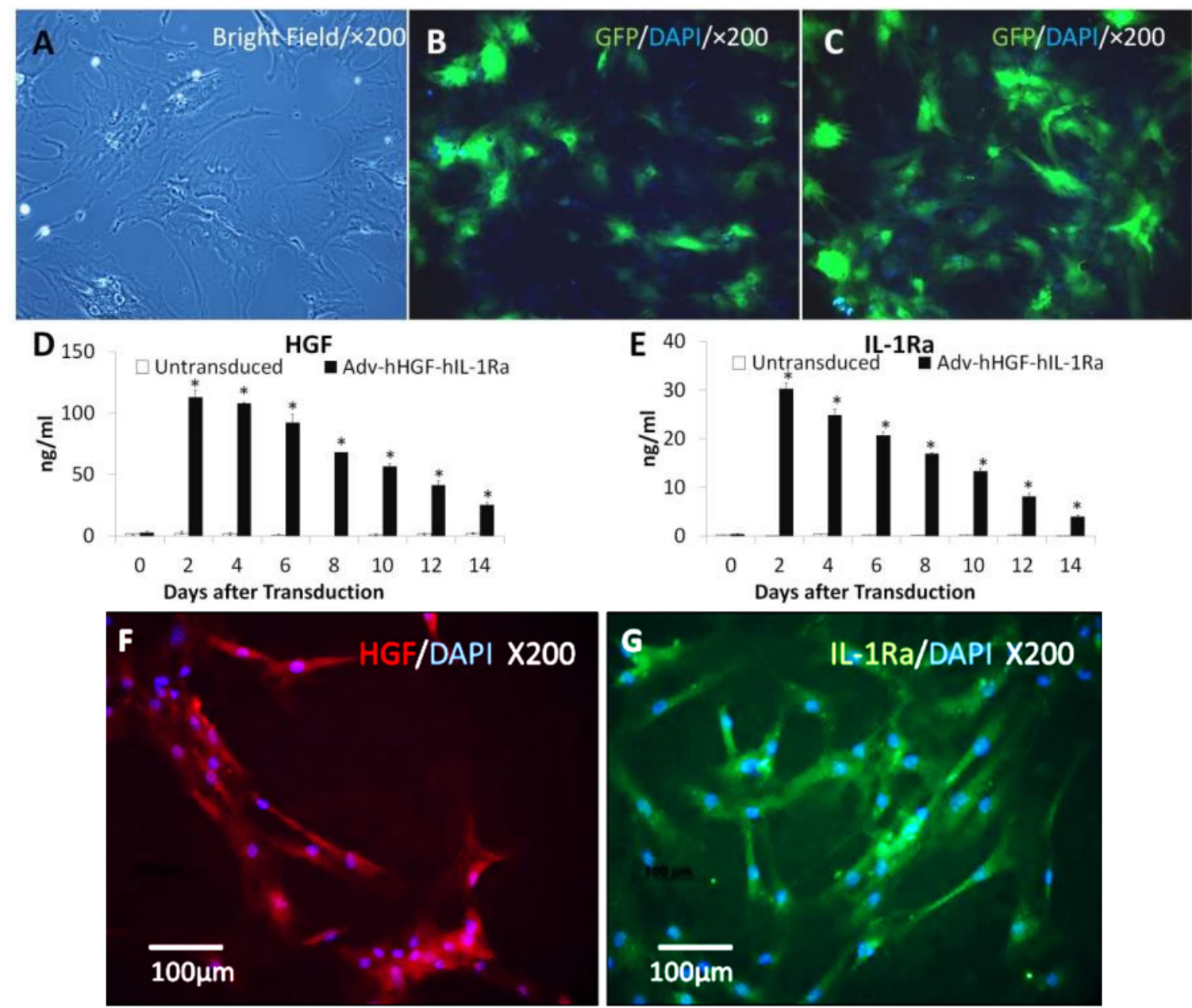

Figure 5-3. Transduction efficiency of Adv-hHGF-hIL-1Ra into BMSCs

A. Primary BMSCs exhibit a spindle-shaped fibroblastic morphology after ex vivo expansion. B. GFP expression in primary BMSCs after the transduction of Adv-GFP at 60 MOI. C. GFP expression in primary BMSCs after the transduction of Adv-GFP at 120 MOI. D. Time profile of HGF gene expression in primary BMSCs after the transduction of Adv-hHGF-hIL-1Ra at 120 MOI as determined by ELISA. E. Time profile of IL-1Ra gene expression in primary BMSCs after the transduction of Adv-hHGF-hIL-1Ra at 120 MOI as determined by ELISA. F-G. Expression of HGF and IL-1Ra in BMSCs after the transduction of Adv-hHGF-hIL-1Ra at $120 \mathrm{MOI}$ as determined by immunofluorescence staining. Data are represented as the mean \pm SD. $n=3 . * P<0.05$ under $t$ test. 
human islets against inflammatory cytokine from Adv-hHGF-hIL-1Ra transduced primary BMSCs in an ex vivo co-culturing study. Results showed that after stimulated with $5 \mathrm{ng} / \mathrm{ml}$ recombinant human IL-1 $\beta$ for 4 days, the cluster-like structures of islets were destroyed and viability of islets decreased (Figure 5-4). However, the viability andmorphological integrity were better preserved in the islets co-cultured with AdvhHGF-hIL-1Ra transduced primary BMSCs. The protection on cultured human islets from Adv-hHGF-hIL-1Ra transduced primary BMSCs even lasted to 4 weeks. To determine whether this protection effect was a result of the Adv-hHGF-hIL-1Ra transduction on BMSCs or the organ repairing function of primary BMSCs, the insulin release profiles over time from islets cultured alone, islets cultured with untransduced primary BMSCs and islets cultured with Adv-hHGF-hIL-1Ra transduced primary BMSCs were monitored (Figure 5-4). Results showed that the insulin releases from islets cultured with primary BMSCs were significantly higher than the islets cultured alone at day 10 of culture, indicating that BMSCs did help to protect islets viability. However, untransduced BMSCs did not provide sufficient protection against the apoptotic islet death caused by IL-1 $\beta$. Results showed that insulin releases from islets cultured with untransduced primary BMSCs decreased dramatically $(75.1 \pm 9.7 \%)$ in 4 days after stimulating with $5 \mathrm{ng} / \mathrm{ml}$ recombinant human IL-1 $\beta$. However, insulin releases from islets cultured with Adv-hHGF-hIL-1Ra-transduced BMSCs decreased only slightly (23.4 \pm $5.6 \%)$.

In summary, these results demonstrated that primary BMSCs supported islet viability and function, but primary BMSCs alone failed provide islets with sufficient protection against inflammatory cytokines in the physiology condition. Adv-hHGF-hIL1 Ra-transduced primary BMSCs effectively prevented the apoptotic islet death caused by inflammatory cytokines.

\section{Co-transplantation of islets with Adv-hHGF-hIL-Ra-transduced BMSCs improved outcome of islet transplantation}

Transplantation of islets under the kidney capsule of diabetic NOD-SCID mice reversed diabetes in every subject. As shown in Figure 5-5, the mice transplanted with 1000 IEs all reverted to diabetes after only 4 weeks. However, the diabetic mice receiving 1000 IEs and Adv-hHGF-hIL-Ra-transduced BMSCs maintained the blood glucose level $\leq 250 \mathrm{mg} / \mathrm{dL}$ for 15 weeks, suggesting prolonged protection of islets by Adv-hHGF-hILRa-transduced primary BMSCs. Results also showed that increasing islet mass from 1000 IEs to 2000 IEs effectively improved the outcome of islet transplantation (Figure 5-5), although rapid increase in blood glucose level was still observed when no additional BMSCs were co-transplanted. We considered mice with blood glucose $\leq 250 \mathrm{mg} / \mathrm{dL}$ as the reversed-diabetes mice to determine the cumulative outcome of islet transplantation. Co-transplantation of islets with Adv-hHGF-hIL-1 Ra-transduced BMSCs significantly increased the reversed-diabetes ratio, suggesting the positive role of Adv-hHGF-hIL-Ratransduced primary BMSCs after islet transplantation. To determine the islet function at 15 weeks after islet transplantation, the mice were anesthetized to collect blood to measure serum insulin and c-peptide level by ELISA. Results showed that the levels of 

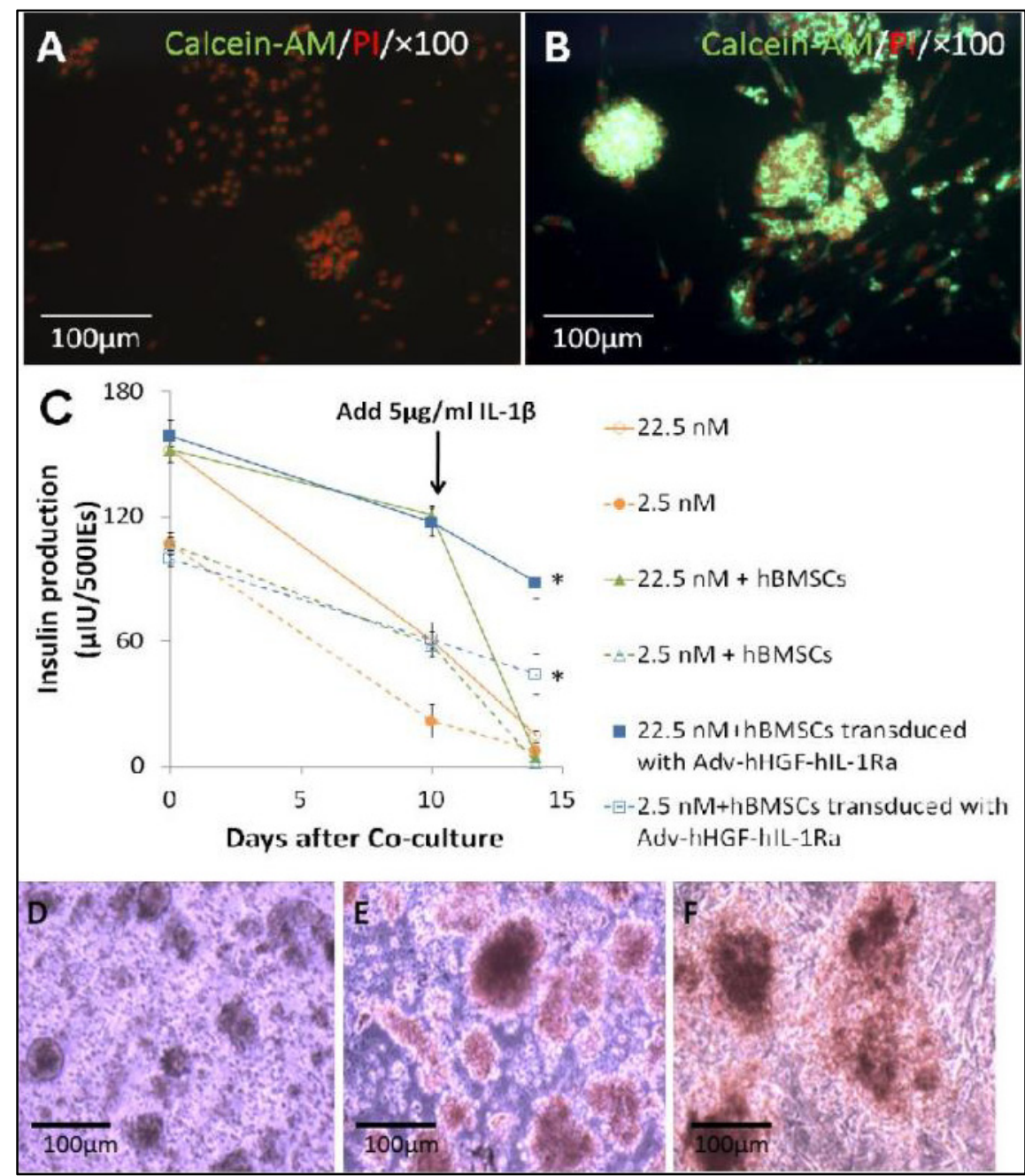

Figure 5-4. Adv-hHGF-hIL-1Ra-transduced BMSCs helped to preserve islet viability against IL-1 $\beta$ stimulation

A. Viability of naked islets after IL-1 $\beta$ stimulation for 4 days, as determined by calceinAM/PI double staining. B. Viability of islets co-cultured with Adv-hHGF-hIL-1Ratransduced BMSCs after IL- $1 \beta$ stimulation for 4 days, as determined by calcein-AM/PI double staining. C. Glucose-stimulated insulin release from islets alone, islets co-cultured with primary BMSCs and islets IEs co-cultured with Adv-hHGF-hIL-1Ra-transduced BMSCs at day 0, day 10, and day 14 after co-culturing, as determined by ELISA. $5 \mu \mathrm{g} / \mathrm{ml}$ IL-1 $\beta$ was added at day 10 (arrow) and incubated with islets for 4 days. Data are presented as the mean $\pm \mathrm{SD}, \mathrm{n}=3 .{ }^{*} P<0.05$ under one-way ANOVA test. D-F. Viability of human islets cultured for 4 weeks alone (bottom left) or with Adv-hHGF-hIL-1Ra transduced primary BMSCs (bottom right) as determined by dithizone staining. Fresh islets were used as positive control (bottom center). 


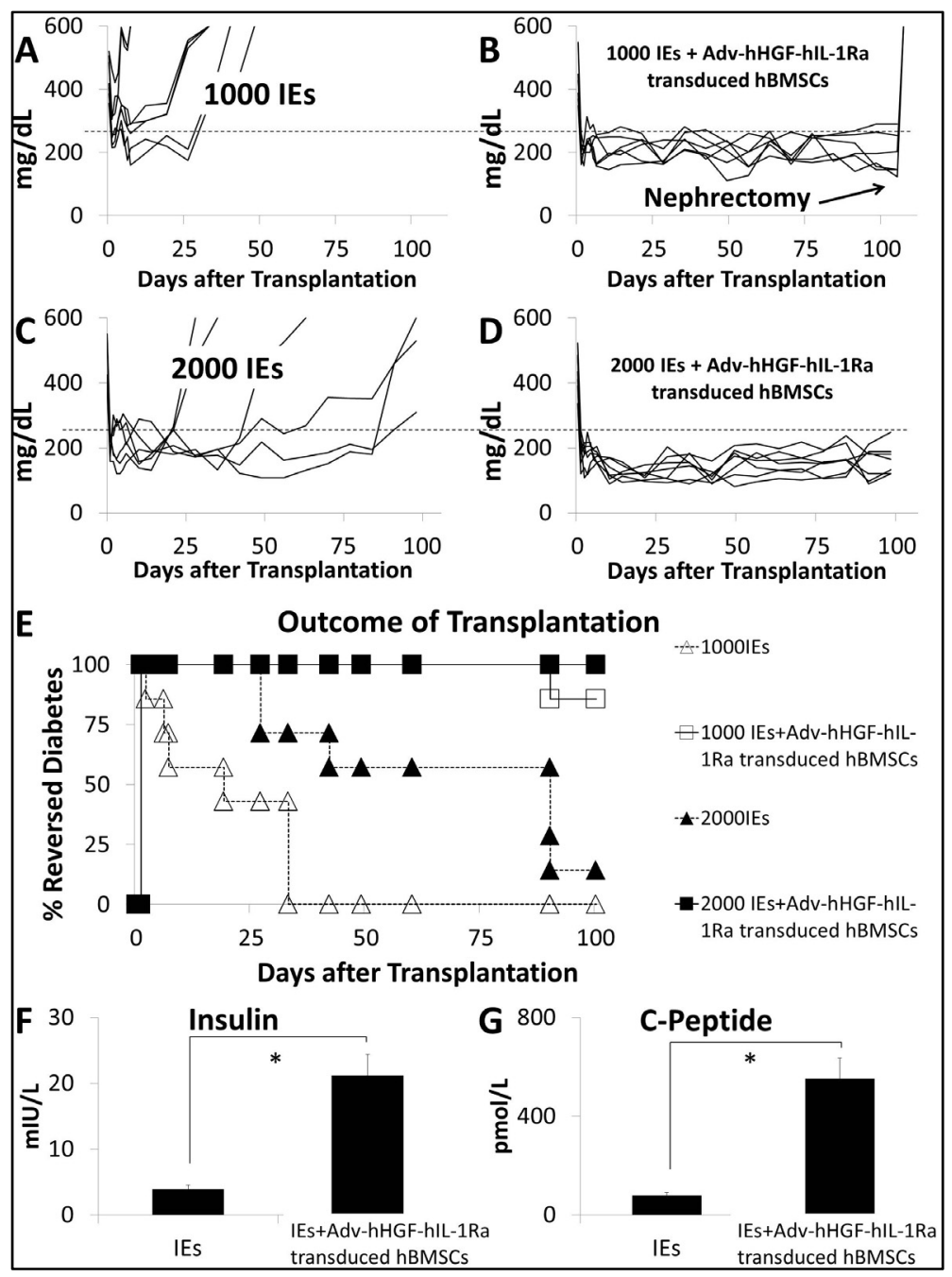

Figure 5-5. Outcome of islet transplantation after being cotransplanted with AdvhHGF-hIL-1Ra-transduced BMSCs

A-D. The blood glucose level of every single mouse after receiving 1000 IEs (A), 1000 IEs co-transplanted with Adv-hHGF-hIL-1Ra-transduced BMSCs (B), 2000 IEs (C) and 2000 IEs co-transplanted with Adv-hHGF-hIL-1Ra-transduced BMSCs (D). Blood glucose $\leq 250 \mathrm{mg} / \mathrm{dL}$ was identified as reversed-diabetes (dashed line). E. Reverseddiabetes ratio of the NOD-SCID mice after islet transplantation. White triangles = mice receiving 1000 IEs, white squares = mice receiving 1000 IEs co-transplanted with AdvhHGF-hIL-1Ra-transduced BMSCs, black triangles = mice receiving 2000 IEs, black squares $=$ mice receiving 2000 IEs co-transplanted with Adv-hHGF-hIL-1Ra-transduced BMSCs. F. Average serum insulin level of the mice receiving 1000 IEs and 1000 IEs cotransplanted with Adv-hHGF-hIL-1Ra-transduced BMSCs 15 weeks after islet transplantation, as determined by ELISA. G. Average serum c-peptide level of the mice receiving 1000 IEs and 1000 IEs co-transplanted with Adv-hHGF-hIL-1Ra-transduced BMSCs 15 weeks after islet transplantation, as determined by ELISA. Data are presented as the mean $\pm \mathrm{SD}, \mathrm{n}=7$. ${ }^{*} P<0.05$ under $\mathrm{t}$ test. 
insulin and c-peptide of the mice transplanted with 1000 IEs co-transplanted with AdvhHGF-hIL-1Ra-transduced primary BMSCs were significantly higher than those of the mice transplanted with islets alone. The body weight was also measured to validate the blood glucose measurement (Figure 5-6). The increases in body weight were significantly slower in mice receiving islets alone since their blood glucose reverted to diabetes sooner after transplantation. However, this phenomenon was not observed in mice receiving islets and Adv-hHGF-hIL-1Ra-transduced primary BMSCs.

Intraperitoneal glucose tolerance test was used to determine islet engraftment and function 2 weeks after transplantation. Results showed faster and better response to the glucose boost in mice receiving 1000 IEs co-transplanted with Adv-hHGF-hIL-1Ratransduced primary BMSCs compared with mice receiving islets alone (Figure 5-7). These results demonstrated the improvement in islet engraftment and function after cotransplantation of islets with Adv-hHGF-hIL-1Ra-transduced primary BMSCs and agreed with recently published results [60].

\section{Adv-hHGF-hIL-Ra-transduced BMSCs promoted islet engraftment and revascularization}

As shown in Figure 5-8, immunofluorescence staining showed the engraftments of insulin-positive islets under the kidney capsule. Qdot labeled BMSCs were detected in proximity of human islets, suggesting a protective role. As shown in Figure 5-9, the staining of vWF was undetectable in the kidney sections bearing islets alone and sparse in the kidney sections bearing islets and untransduced primary BMSCs. However, evident vWF staining in the kidney sections bearing islets co-transplanted with Adv-hHGF-hIL1Ra-transduced primary BMSCs was detected at both 4 weeks and 15 weeks after islet transplantation. After analyzed the relative $\mathrm{vWF}$ intensity from three independent regions using ImageJ, these results suggested increased revascularization by Adv-hHGF-hIL1Ra-transduced primary BMSCs.

\section{Discussion}

The purpose of this study was to combine MSC based cell therapy with adenovirus based gene therapy to improve the outcome of islet transplantation. We have been working on Adv based gene therapy for years. Replication-deficient Adv vectors are extensively reported to be effective in blocking the inflammatory cytokine pathway and increasing the islet resistance to post-transplantation challenges [4]. HGF is known to induce angiogenesis, promote $\beta$-cell proliferation and protect islets from apoptosis and eventually helps to improve the transplantation outcome in STZ-induced diabetic mice [9, 122]. IL-1Ra binds to the cell surface interleukin-1 receptor and prevents IL-1 from sending a signal to that cell. Ex vivo transduction of human islets with Adv-hIL-1Ra has been reported to prevent IL-1 $\beta$ cell induced apoptotic cell death in islets [3]. We previously reported the improved islet transplantation using Adv-hHGF-hIL-1Ra transduced human islets [8]. However, most Adv vector failed to support the long-term 


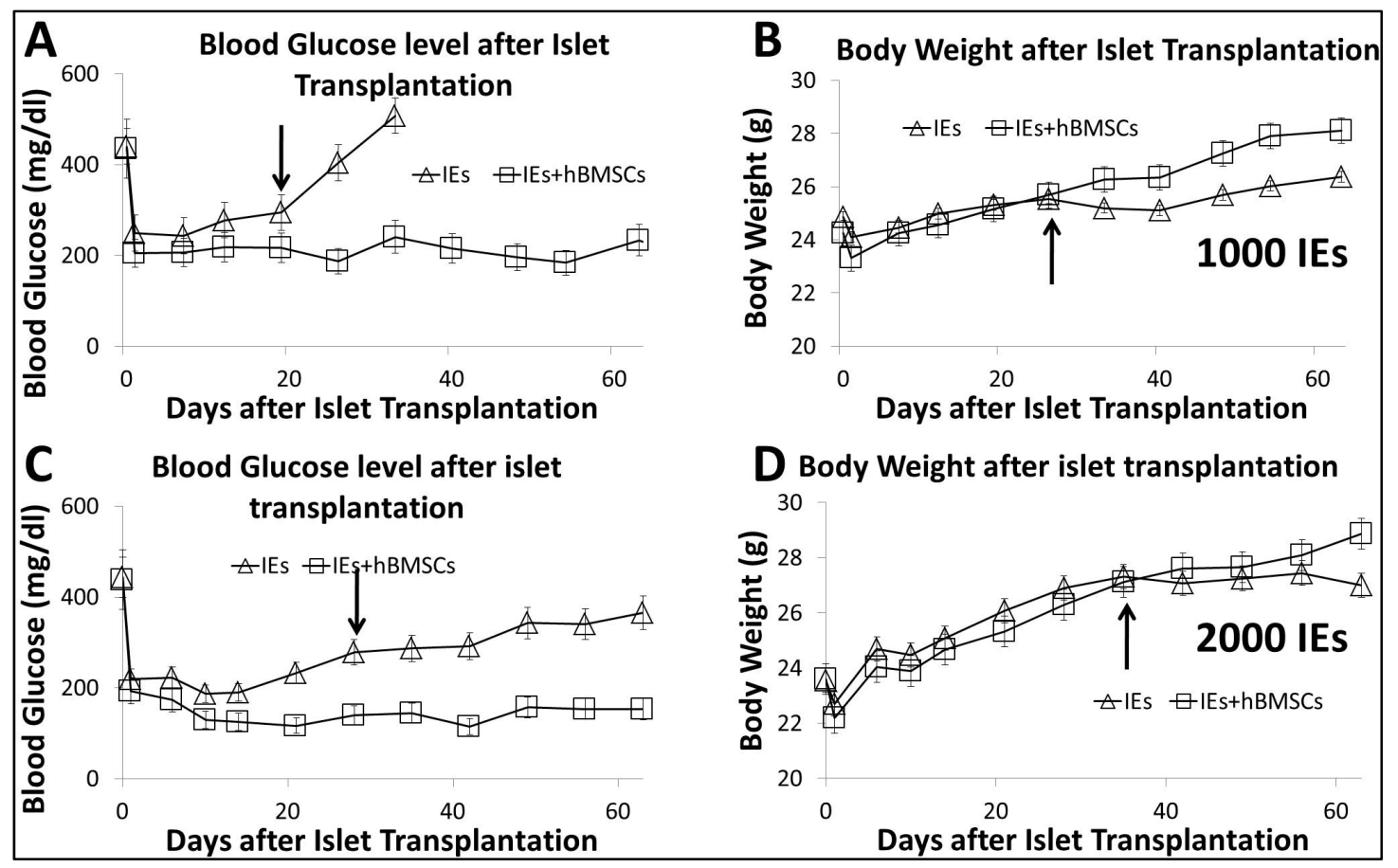

Figure 5-6. Body weight and blood glucose after human islet transplantation A-B. The average blood glucose level (A) and average body weight (B) of mice receiving 1000 IEs and 1000 IEs co-transplanted with Adv-hHGF-hIL-1Ra-transduced BMSCs (B). C-D. The average blood glucose level (C) and average body weight (D) of mice receiving 2000 IEs and 2000 IEs co-transplanted with Adv-hHGF-hIL-1Ra-transduced BMSCs (D). Decreasing average weight gain was observed in the mice receiving islets alone sooner after the average blood glucose reversed back to diabetic level (arrow). However, this phenomenon was not observed in mice receiving islets and Adv-hHGF-hIL-1Ratransduced BMSCs. 


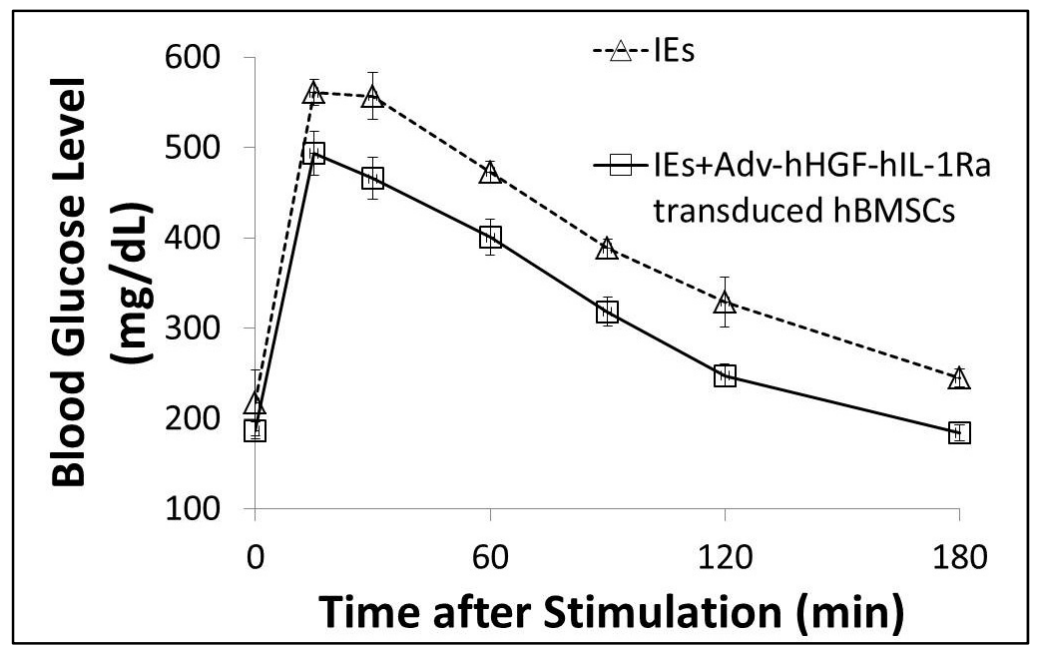

Figure 5-7. Intraperitoneal glucose tolerance test of the mice receiving 1000 IEs and 1000 IEs co-transplanted with Adv-hHGF-hIL-1Ra-transduced BMSCs at 2 weeks after islet transplantation

Data are presented as the mean $\pm \mathrm{SD}, \mathrm{n}=7$. ${ }^{*} \mathrm{P}<0.05$ under $\mathrm{t}$ test. 


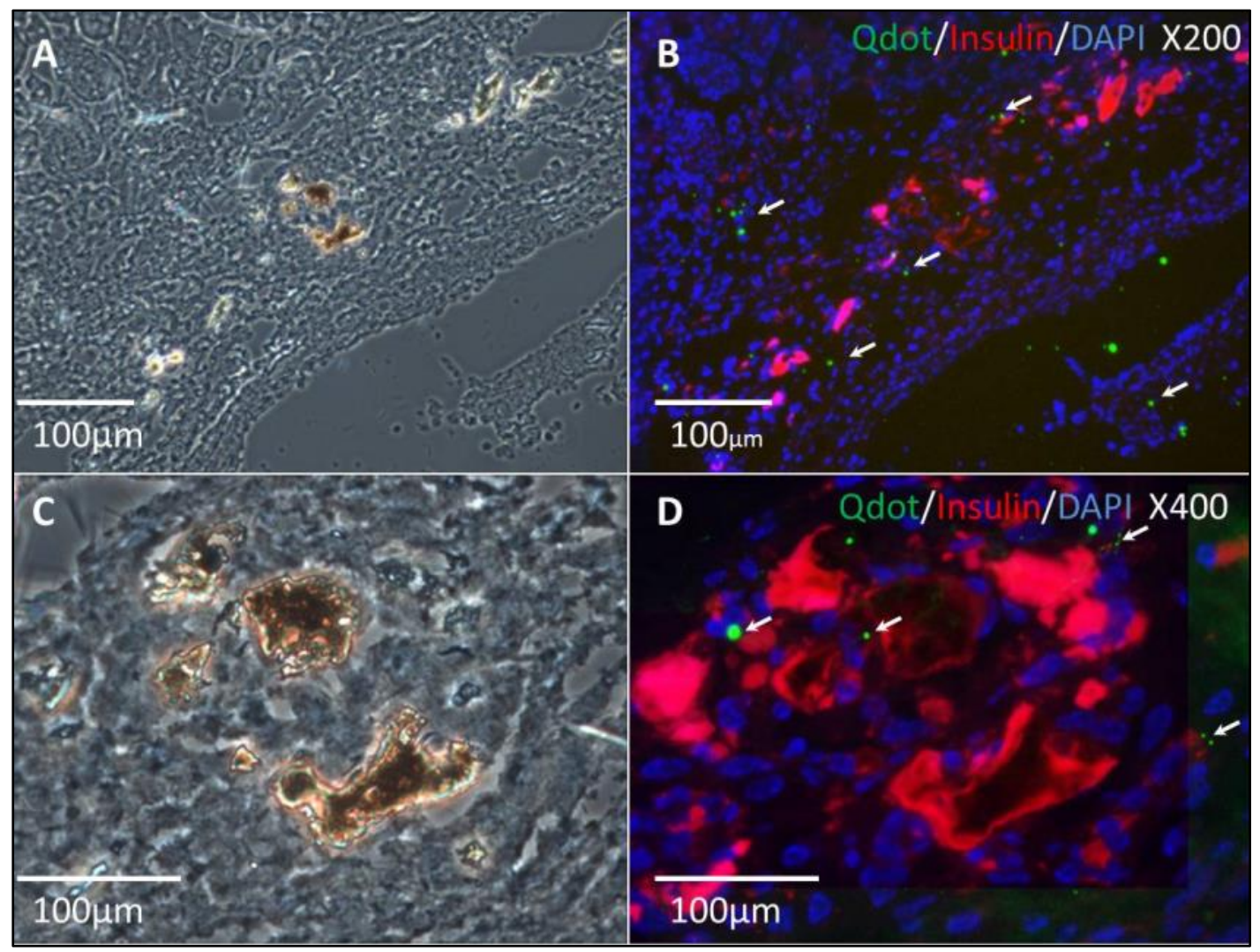

Figure 5-8. Immunofluorescence staining of the kidney section bearing islets and Adv-hHGF-hIL-1Ra-transduced BMSCs

A.B. Images under 20x magnification from bright field (A) and fluorescent microscope (B). C.D. Images under 100x magnification from bright field (C) and fluorescent microscope (D). Insulin was stained in red to indicate the functional human islets. Qdot 565 labeled BMSCs were green "blinking" dots in close proximity of transplanted islets (white arrows). 


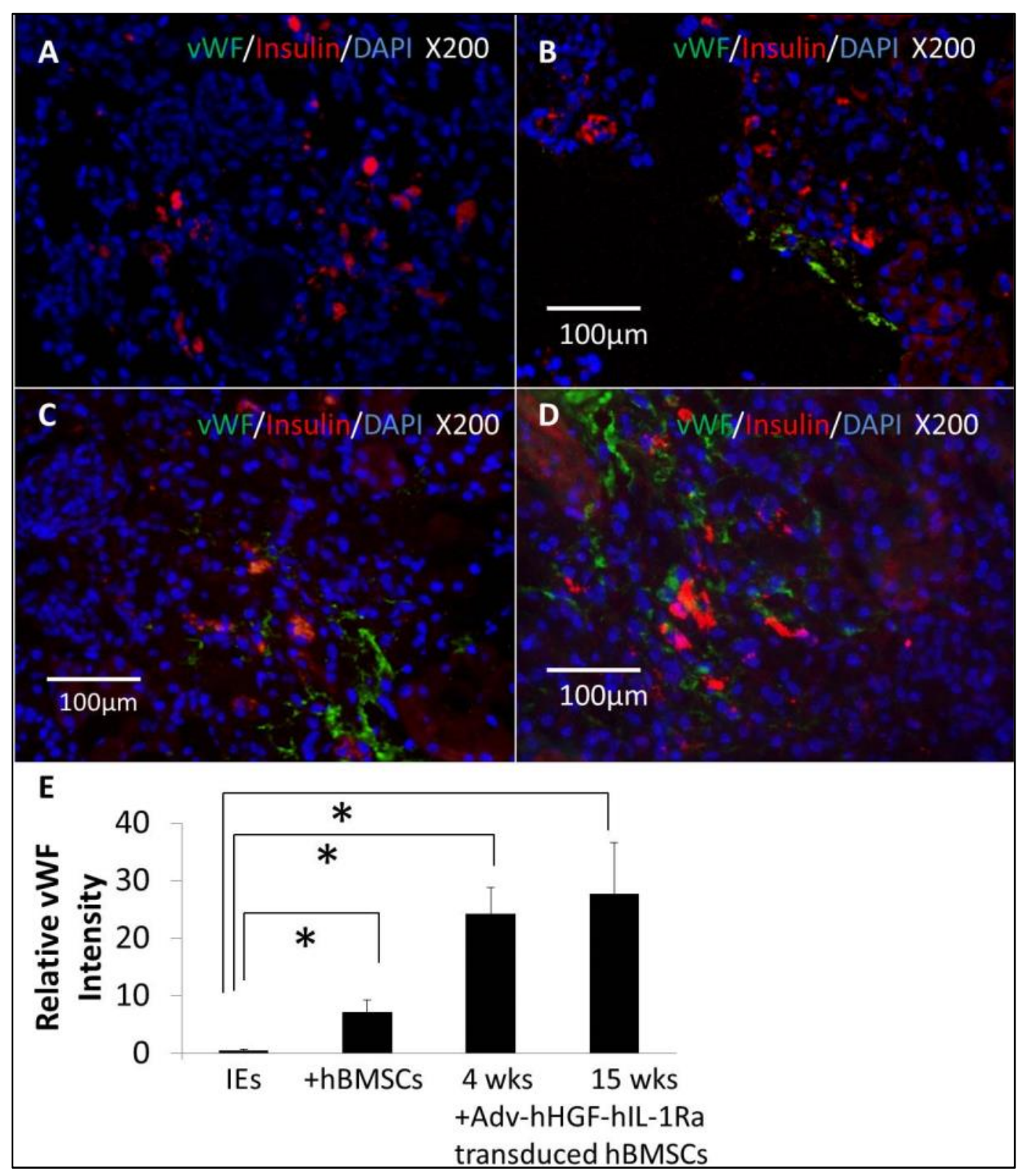

Figure 5-9. Adv-hHGF-hIL-1Ra-transduced BMSCs promoted revascularization of the transplanted islets

A-D. Representative staining of vWF factor (green) in the kidney bearing islets alone at 4 weeks after transplantation (A), the kidney bearing islets co-transplanted with untransduced primary BMSCs at 4 weeks after transplantation (B), the kidney bearing islets co-transplanted with Adv-hHGF-hIL-1Ra-transduced primary BMSCs at 4 weeks after transplantation (C) and the kidney bearing islets co-transplanted with Adv-hHGFhIL-1Ra-transduced primary BMSCs at 15 weeks after transplantation (D). E. vWF fluorescent intensity was quantified using Image $J$ from three independent regions. Data are presented as the mean $\pm \mathrm{SD}, \mathrm{n}=3, * P<0.05$ under $\mathrm{t}$ test. 
survival of islet grafts, probably due to the short expression profile of Adv. For example, the expression of HGF and IL-1Ra drastically decreased at 14 days after Adv transduction (Figure 5-3). Lentivrus could be an alternative solution for long term gene therapy. But since lentivirus integrates into the host genome and has the potential for generation of replication competent lentivirus and oncogenesis [123], great caution should be used before starting in this area.

Another problem with Adv based gene therapy is the viral genome, more or less, co-expressed with target genes may trigger an increasing immune response to the transplanted organs. Some reports suggested that Adv transduction led to immune reaction to the Adv-bearing organs and made them more susceptible to attack from the recipient's immune system with potentially dangerous consequences [124, 125]. Therefore, how to find the perfect vehicles to provide prolonged protection to islet grafts with less immunogenicity is a critical question to be answered by traditional gene therapy.

BMSCs seem to be one of these perfect vehicles for gene delivery. BMSCs are pluripotent adult stem cells with the property of self-renewal and the potential to differentiate into many types of cells [40]. They are open to genetic manipulation and capable of secreting endogenous growth factors to promote engraftment of human islets $[58,126]$. They are also hypoimmunogenic, probably by releasing anti-inflammatory cytokines and inhibiting T cell proliferation and activation $[127,128]$. All these characteristics make BMSCs good nursing cells to advance current clinical studies of islet transplantation by combining gene therapy with cell therapy.

However, probably because of the lack of standard operating protocols for isolating, preserving, and culturing BMSCs, the supporting role of BMSCs is still under discussion. The secretion of growth factors such as HGF, VEGF, and stem cell factor by BMSCs is controversial. We did not detect SCF expression by primary BMSCs, and the basal expression of HGF and VEGF from primary BMSCs was too low to support an optimal revascularization for islet graft survival (Figure 5-8) as noted in previous reports [129]. We demonstrated that exogenous expression of HGF by Adv-hHGF-hIL-1Ratransduced primary BMSCs could be maintained for at least 2 weeks and may eventually lead to the notable increasing revascularization of islet grafts after transplantation (Figure 5-3).

Despite many reports on hypoimmunogenic nature of BMSCs, its mechanism is unclear. There are two hypotheses: 1) through the secretion of immunosuppressive cytokines, such as IL-10 and TGF $\beta$ [130]; and 2) through cell surface molecules of BMSCs to inhibit T cell activity and proliferation [71]. Previous reports showed that the co-transplantation of islets with primary BMSCs prevented the allogeneic rejection and helped engraftment of islets, but one common feature in all these studies was that the BMSCs were incompetent to reverse the blood glucose levels of recipients in the acute stage ( $<3$ weeks) after transplantation, suggesting the slow engraftment of transplanted islets $[43,54,116]$. To make the situation even worse, the hyperglycemia caused by slow engraftment in turn accelerated the apoptotic islet cell death [95]. We hypothesized that 
this phenomenon was probably due to the incapability of primary BMSCs to protect islets against the inflammatory-cytokine induced apoptotic cell death. In consistence with previous report [131], we demonstrated that the native secretion of IL-10 by BMSCs was insufficient to support islet survival against the inflammatory cytokine (Figure 5-4). We also demonstrated that Adv-hHGF-hIL-1Ra transduced primary BMSCs has clear advantaged in preventing inflammatory-cytokine induced islet death compared with untransduced primary BMSCs (Figure 5-4).

BMSCs are hypoimmunogenic cells in lack of the major histocompatibility complex class II (MHC II) [56], making them capable of escaping the recognition by Natural Killer cells and alloreactive T cells. This is an important feature for the gene therapy to improve islet transplantation because 1) adding primary BMSCs into islets will not significantly raise allogeneic rejection, and 2) BMSCs could serve as gene delivery vehicles to avoid the notable immunogenicity of Adv vectors. Primary BMSCs were also capable of repairing injured tissues in long term [132]. We demonstrated that when islets were co-transplanted with Adv-hHGF-hIL-Ra transduced primary BMSCs, the blood glucose levels of diabetic NOD-SCID mice were maintained $\leq 250 \mathrm{mg} / \mathrm{dL}$ for more than 15 weeks (Figure 5-5), significantly longer than most reported islet transplantations using genetic modified islets $[4,16,133]$. These results indicated the advantage of primary BMSCs as gene delivery vehicles to improve the outcome of islet transplantation. Although an immunocompetent animal model should be used to further validate the benefit of hypoimmunogenic primary BMSCs as gene delivery vehicles, our work provided useful information for further research.

The differentiation capability of BMSCs is another interesting feature worthy of mentioning. The capacity of BMSCs to differentiate into a variety of cells including osteoblasts, adipocytes and chondrocytes and so on is one of most important mechanisms underlying its capability of tissue repair [132]. Moreover, viral transduced BMSCs still retained their differentiation capacity $[134,135]$. This tissue repairing capacity of primary BMSCs, as supported by majority of reports, may eventually lead to the long term protection on human islets and was further supported by the fact that BMSCs were in close proximity to the islet grafts (Figure 5-8). Other reports showed that BMSCs have the potential to differentiate into vascular endothelial cells [136]. This is also possible because revascularization was observed mostly around islet grafts where most BMSCs settled (Figure 5-9). However, whether the neoforming blood vessels in the kidney bearing islet grafts is from BMSCs alone or other cell types remains to be clarified.

In agreement with other reports on islets/BMSCs co-transplantation [67, 137], our results indicated that the co-transplantation of islets with Adv-hHGF-hIL-1Ra-transduced primary BMSCs into diabetic NOD-SCID mice promoted local revascularization, improved islet viability and reversed diabetes in long term. We are the first group to report the combination strategy of Adv-based gene therapy and BMSC-based cell therapy to improve the outcome of islet transplantation. Our data indicated that BMSCs were efficient gene delivery vehicles to improve the outcome of islet transplantation, as they did to treat other diseases $[138,139]$. Adv-hHGF-hIL-1Ra-transduced primary BMSCs secreted elevated levels of HGF and IL-1Ra and retained their protective capability for 
the long-term survival of islet grafts. Since current gene therapy approaches still focus on the expression of endogenous growth factors in human islets, our report provides a novel direction for future exploration of other factors and cells for co-transplantation. Our studies in rodents also provide useful information on the future studies on the immunocompetent animal model. However, several issues still need clarification before this approach can be clinically applicable, including the immunogenicity and in vivo fate of Adv-transduced BMSCs, mechanism underlying the long-term protection of BMSCs, and the optimal ratio between islet and BMSC numbers.

In our previous and present studies $[6,8]$, most transplanted human islets survived only up to $4 \sim 6$ weeks in diabetic NOD-SCID mice without any treatment. We can also find out similar length of islet survival in other reports $[140,141]$. We are aware of several reports of islet survival beyond 60 days post-transplantation. This discrepancy is due to the fact that islet survival and function post-transplantation depends on multiple factors, including donor condition, islet purity, immunocytes, hyperglycemia, hypoxia and inflammation after transplantation. The degree of diabetes and variability in mice used for transplantation also greatly affect the length of islet survival and function. The other reason may be the pre-transplantation treatment after islet isolation. When handpicked islets were used for transplantation, the length of islet survival was significantly improved, possibly due to discarding of lymph nodes in the hand-picking process as the existence of lymph nodes is detrimental to the survival of islets after transplantation. However, we are not personally in charge of the isolation process and we do not prefer to use hand-picked islets for transplantation.

In summary, co-transplantation of islets and BMSCs promoted revascularization, protected islet viability, and significantly reduced the islet mass required for reverse diabetes. The production of HGF and IL-1Ra by Adv-hHGF-hIL-1Ra-transduced primary BMSCs and the protective capability of BMSCs synergistically helped to improve the islet survival in the early stage and graft function in the long term run after transplantation. 


\section{CHAPTER 6. BMSC PREVENTS ALLOGRAFT REJECTION OF HUMAN ISLET TRANSPLANTATION IN HUMANIZED MOUSE MODEL*}

\section{Introduction}

We marched a long trip to find the best ways to induce diabetes, to purify human islets and to transplant human islet under the kidney capsule of diabetic mice. However, the absence of human immunity in all the animal experiments has rendered all the previous efforts far from clinical human islet transplantation. In fact, human islets can only settled in immunodeficient animals for longer time ( $>4$ weeks) and the absence of human immunity is a common weakness of all studies involving human islet transplantation in diabetic animals. These studies were actually focused on the PNF, which is the loss of human islets caused by non-immune challenges including inflammatory cytokines, hypoxia, ROS and poor revascularization, instead of immune rejection.

To introduce immune rejection into preclinical studies regarding human islet transplantation, some groups have been using immunocompetent rodents [60, 142]. However, the immune responses from mice and human are drastically different and the results from these studies must be interpreted with caution. Recently, the development of humanized mouse marked a huge advance in the animal models used for human islet transplantation [79]. First, it is a perfect model to study the development of type 1 diabetes, which is an autoimmune disease. Second, the interaction between transplanted islets and the adoptively transferred human immunity provide useful information about immune rejection after human islet transplantation. The human immunity can be established in several specialized mouse strains by injection of human immunocytes validated by the presence of human IgG in the blood steam and CD3+ T cells in the mouse spleen [79]. The adoptively transferred human immunity posed a challenge, which is similar to the clinical immune rejection, to the transplanted human islets.

Compared with previous studies using immunodeficient animals [52, 54], the duration of insulin independence in humanized mice was significantly reduced [79], suggesting the existence of immune rejection. However, the introduction of human immunity also led to GvHD, a fetal disease characterized by the attack to the recipient's major organs by the infused xenogeneic immunocytes. The risk of GvHD can be controlled by reducing the amount of the infused immunocytes and even eradicated by generating humanized mice using hematopoietic stem cells [79].

The potential of BMSC as a novel personalized medicine has inspired us to explore its potential as an immune modulator. Although the recent improvements in the

\footnotetext{
* Reprinted with permission from Nature Publishing Group. Wu, H, Wen, D, and Mahato, RI (2013). Third-Party Mesenchymal Stem Cells Improved Human Islet Transplantation in a Humanized Diabetic Mouse Model. Molecular Therapy, (in press).
} 
regimen of immunosuppressive drugs such as tacrolimus, sirolimus and MPA have greatly reduced their side effects, these drugs are still toxic to the viability and function of transplanted islets. BMSC on the other hand, which can be easily isolated from a diabetic patient and expanded in vitro, does not cause such concerns.

Unlike Gene therapy, which is incapable to prevent the immune rejection, stem cell therapy has recently met great success as a novel regenerative medicine to support solid organ transplantation including human islet transplantation [52, 143]. Among all types of stem cells, BMSCs are given special interest for their self-renewal potential, multi-lineage capacities, paracrine effects (trophic mediator) and immune modulatory effects $[40,55]$, making it a great candidate for improving human islet transplantation.

MSC-based therapy has been used to improve human islet transplantation from several aspects. Ding et al. reported the immunosuppressive effects of MSCs by secreting soluble factors [60]. Korbutt et al. reported that co-culture with MSCs improved islet viability and function against inflammatory cytokines [144]. Ito et al. and other researchers reported that MSCs were capable of promoting the revascularization of islets in vivo $[52,54]$. However, the translation to a real clinical breakthrough is slow and the data from limited clinical trials seems vague and controversial, probably due to the lack of the human immunity in most of the previous studies. Hereby we evaluated the effectiveness of BMSCs from a third party donor to improve the outcome of human islet transplantation in a diabetic humanized NSG mouse model. We plan to answer two questions by this study: 1) the interaction of BMSCs with adoptively-transferred human immunity to protect islets in vivo; 2) the tropic effect of BMSCs to support islet function.

\section{Materials and Methods}

\section{Materials}

NSG and NOD-SCID mice were purchased from Jackson Laboratory and housed in pathogen-free microisolator cages. Human PBMCs were isolated from fresh buffy coats (Lifeblood South, Memphis, TN) by gradient centrifugation using Ficoll-Paque (GE Healthcare, Little Chalfont, UK). Before use, the preparations of PBMCs were characterized by FlowCellect ${ }^{\mathrm{TM}}$ Human Lymphocyte ZAP-70 Characterization Kit (Millipore, Billerica, MA). To establish human immunity, PBMCs were injected intraperitoneally into NSG mice (5x106/mouse) as described before [145]. The settlement of T cells in the spleen was assessed two weeks after injection by immunofluorescence staining. The function of B cells was assessed by measuring human IgG concentration in the mouse blood. Human islets were received from Integrated Islet Distribution Program (Duarte, CA). Primary BMSCs were purchased from Thermo Fisher Scientific (Waltham, MA), passaged, and frozen in our lab. Before use, BMSCs were characterized of the surface markers including human leukocyte antigens (HLA)-DR, HLA class I, CD14, CD80, CD86 and Fas Ligand (FasL or CD95L) (Abcam, Cambridge, MA). 


\section{Mixed lymphocyte reaction (MLR)}

The proliferation of PBMCs was determined using CFSE proliferation assay. CFSE passively diffuses into cells, retained by the cells throughout division and can be used to differentiate the successive generations from the parental generations. Briefly, CFSE-labeled PBMCs (1x106) were stimulated with phytohaemagglutinin (PHA, $1 \mu \mathrm{g} / \mathrm{mL})$ for $24 \mathrm{~h}$ and co-cultured with BMSCs $(5 \times 104)$ in the same well or in a transwell system for indicated time. In the transwell system, the BMSCs were adherent to the bottom of the cell culture plate while the PBMCs were cultured in a $0.4 \mu \mathrm{m}$ transwell cell culture inserts (Corning, Tewksbury, MA). The media was carefully monitored to rule out the penetration of the PBMCs from the inserts. The division history of PBMCs was analyzed by flow cytometry. The percentage of proliferated cells was calculated by dividing the number of cells undergoing divisions with the number of total cells in the cultures. The total number of PBMCs was measured using T4 Automatic Cell Counter (Nexcelom, Lawrence, MA). The extent of T cell activation was determined by measuring the level of IL-2, IL-2sRa and IFN- $\gamma$ in the medium using ELISA. IL-10 level in the medium was also determined by ELISA. Total T cells, CD4+ T cells, CD19+ B cells and CD14+ monocytes were isolated or depleted from PBMCs using specific Dynabeads for each population, respectively (Invitrogen, Carlsbad, CA). The mRNA levels of IL-10 in each subpopulation were determined by Realtime PCR and normalized by the levels of $\beta$-actin. The percentage of CD4+CD25+FoxP3+ Tregs was determined by Human Regulatory T cells 3-color kit (RnD System, Minneapolis, MN) using flow cytometry. To study whether a direct contact between BMSCs and PBMCs was required for the immunosuppression of BMSCs, a transwell system was set up.

\section{Islet viability study}

Primary BMSCs $\left(5 \times 10^{5}\right)$ were co-cultured with 500 islets in a $10 \mathrm{~cm}$ dish of CMRL-1066 medium with 10\% FBS for 2 days. Then, the islet/BMSC co-culture was stimulated with cytokine cocktail of TNF $\alpha(50 \mathrm{ng} / \mathrm{mL}), \mathrm{IL}-1 \beta(5 \mathrm{ng} / \mathrm{mL})$ and IFN- $\gamma(50$ $\mathrm{ng} / \mathrm{mL}$ ) for 4 days and the insulin secretion from human islets was quantified using a dynamic islet perifusion assay. Briefly, 50 islets from each group were handpicked and loaded onto a Swinnex 13 chamber (Millipore, Burlington, MA, USA) and perifused with Krebs-Ringer bicarbonate HEPES buffer of the following composition (in mM): 129 $\mathrm{NaCl}, 5 \mathrm{NaHCO} 3,4.8 \mathrm{KCl}, 1.2 \mathrm{KH} 2 \mathrm{PO} 4,1.2 \mathrm{MgSO} 4,2.5 \mathrm{CaCl}$, and $10 \mathrm{HEPES}$ at $\mathrm{pH}$ 7.4. The flow rate was maintained at $1 \mathrm{ml} / \mathrm{min}$ with a peristaltic pump (Themo Fisher, Waltham, MA) and the temperature was maintained at $37^{\circ} \mathrm{C}$ with a solution heater (Warner Instruments, Hamden, CT). Islets were first perifused with basal glucose $(1.67 \mathrm{mM})$ for $60 \mathrm{~min}$ and stimulated with glucose $(16.7 \mathrm{mM})$ for $30 \mathrm{~min}$ and finally perifused with basal glucose $(1.67 \mathrm{mM})$ until insulin release reversed to the basal level. Samples were collected once per two minutes through an automatic fraction collector (Waters, Milford, MA) and analyzed for insulin content by ELISA. 


\section{Islet transplantation}

A long-term low-dose STZ injection was used to induce diabetes in NSG mice. Briefly, STZ $(70 \mathrm{mg} / \mathrm{kg}$ ) was injected intraperitoneally to NSG mice in the first day the first and third week. Animals were mostly diabetic after three weeks as indicated by two consecutive measurements of blood glucose $\geq 400 \mathrm{mg} / \mathrm{dl}$ using a glucometer. Then 500 handpicked human islets $(150 \sim 300 \mu \mathrm{m}$ in diameter) were co-transplanted with primary BMSCs at serial ratios (islet: $\mathrm{BMSC}=1: 0,1: 50,1: 100$ and 1:200) under the kidney capsule of diabetic NSG mice. The nonfasted glucose levels and body weights of all the mice were measured from the pricked tail of each animal up to 15 weeks after transplantation. PBMCs (5x106/mouse) were infused intraperitoneally into these mice at 4 weeks after islet transplantation. At the end of study (17 weeks after islet transplantation), the graft-bearing kidneys were removed from some mice to confirm the function of islet grafts by the return of blood glucose levels to $\geq 400 \mathrm{mg} / \mathrm{dL}$ for 2 consecutive days and all mice were subjected to euthanasia. Serum was collected serum to measure the level of human insulin and c-peptide by ELISA. Whole blood was collected and human CD4+ T cells were isolated using Dynabeads. The percentage of CD4+CD25+FoxP3+ Tregs was determined by flow cytometry.

\section{Intraperitoneal glucose tolerance test}

Two weeks after islet transplantation, glucose tolerance was analyzed in overnight fasted mice as described by Garcia-Ocana et al. [9]. Briefly, the mice were subjected to intraperitoneal injection of glucose at $2 \mathrm{~g} / \mathrm{kg}$ of body weight. Blood samples were obtained from the snipped tail at 15,30,60, 90, 120, and 180 min after injection and analyzed for glucose levels using a glucometer.

\section{Immunofluorescence staining}

Mice receiving islets alone and islets co-transplanted with primary BMSCs were sacrificed 4 weeks after transplantation. The kidneys bearing islets were isolated, washed with PBS, fixed in 4\% paraformaldehyde overnight, and embedded in optimal cutting temperature compound. Frozen sections of $5 \mu \mathrm{m}$ thickness were cut. To detect insulinpositive human islets, the slides were stained with guinea pig anti-insulin primary antibody $(1: 200)$ at $4{ }^{\circ} \mathrm{C}$ overnight and Alexa Fluor 568 conjugated goat anti-guinea pig secondary antibody (1:500) at room temperature for $1 \mathrm{~h}$. To detect CD3+ T cells, the slides were stained with rabbit anti-CD3 primary antibody $(1: 500)$ at $4{ }^{\circ} \mathrm{C}$ overnight and Dylight 488-conjugated goat anti-rabbit secondary antibody (1:500) at room temperature for $1 \mathrm{~h}$. Slides were counter-stained with DAPI. Three independent images were analyzed using ImageJ to quantify the fluorescence intensity. 


\section{Statistical analysis}

Statistical significance of the difference between the two groups was determined by unpaired t-test and between several groups by one-way ANOVA.

\section{Results}

\section{Generation and characterization of humanized NSG mice}

Human PBMCs were isolated from human buffy coat using Ficoll Paque and gradient centrifugation. Typically $1 \times 10^{9}$ of PBMCs can be isolate from $60 \mathrm{~mL}$ of buffy coat. A detailed isolation protocol is listed in the appendix. PBMCs isolated from concentrated human buffy coat was composed of T lymphocytes ( $25 \%)$ and B lymphocytes $(\sim 40 \%)$ (Figure 6-1A). To further isolate a subpopulation of PBMCs, specific dynabeads for each population can be used. Potent human IgG in the mouse blood stream and evident human CD3 $+\mathrm{T}$ cells in the spleen sections were detected at around two weeks after the intraperitoneal injection of PBMCs $(5 \times 10 \%$ mouse), suggesting the successful establishment of human immunity (Figure 6-1B-C). In contrast, human IgG and CD3+ T cells were not detected in NOD-SCID mice because NOD-SCID mice expelled the xenogeneic immunocytes constantly [79].

\section{BMSCs are fibroblast like adult stem cells}

Primary BMSCs exhibit a spindle-shaped fibroblastic morphology after ex vivo expansion. To understand the immunomodulatory effects of BMSCs, we decided to characterize the surface markers of BMSCs. HLA Class I and HLA-DR (class II) were tested because BMSCs might act as antigen presenting cells. We also tested CD14, CD80 and CD86 which are important co-stimulatory factors. Besides, FasL was also tested because they might participate in the apoptosis of T cells [146]. The BMSCs maintained in our lab were positive for HLA class I and negative for HLA-DR, FasL, CD14, CD80 and CD86 (Figure 6-2). However, the lack of co-stimulatory molecules such as CD80 and CD86 suggested that the antigen presentation through BMSCs led to the reversal of T cell maturation and subsequent immune tolerance as suggested by previous literatures [147].

\section{BMSCs suppressed the activation and proliferation of PBMCs}

PBMCs were isolated from human buffy coats. We first tested the immunomodulatory effect of BMSCs on PBMCs in a MLR. Carboxyfluorescein diacetate succinimidyl ester (CFSE), a fluorescent dye retained within cells for a long period, was used to stain PBMCs before co-culturing with BMSCs. The PBMC/BMSC co-culture was stimulated with phytohaemagglutinin (PHA) $(5 \mu \mathrm{g} / \mathrm{mL})$ and allowed to proliferate for 2 days. The daughter cells were determined by the progressive halving of CFSE 


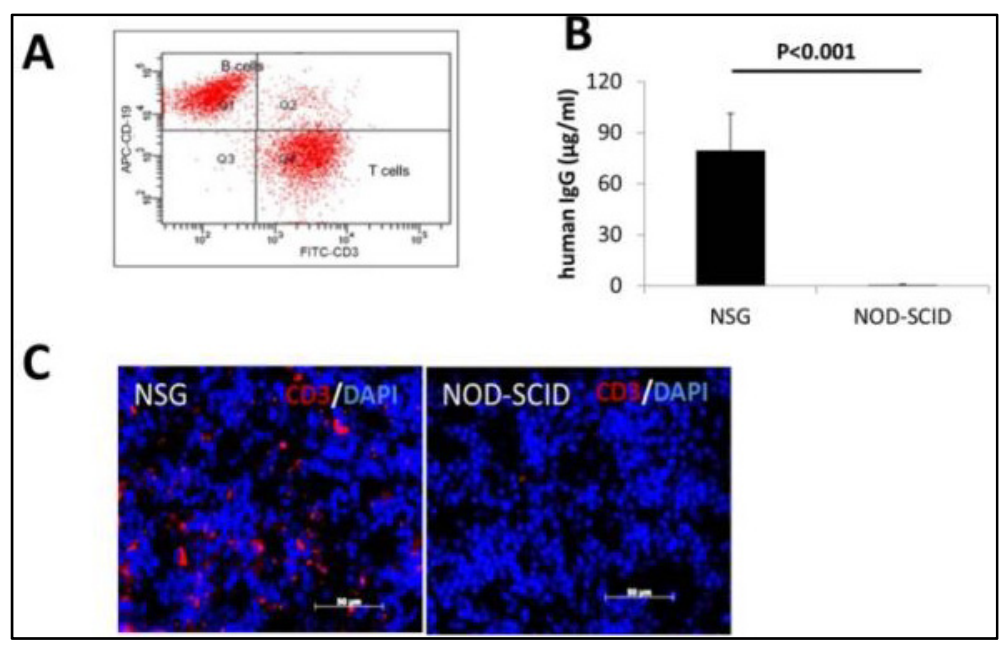

Figure 6-1. Establish human immunity in NSG mice using human PBMCs A. Isolation and characterization of PBMCs from fresh buffy coat. B-C. The serum human IgG concentration (B) and the staining of human CD3+ T cells in the spleen sections (C) of NSG mice and NOD-SCID mice at 2 weeks after the injection of PBMCs $\left(5 \times 10^{6} /\right.$ mouse $)$.

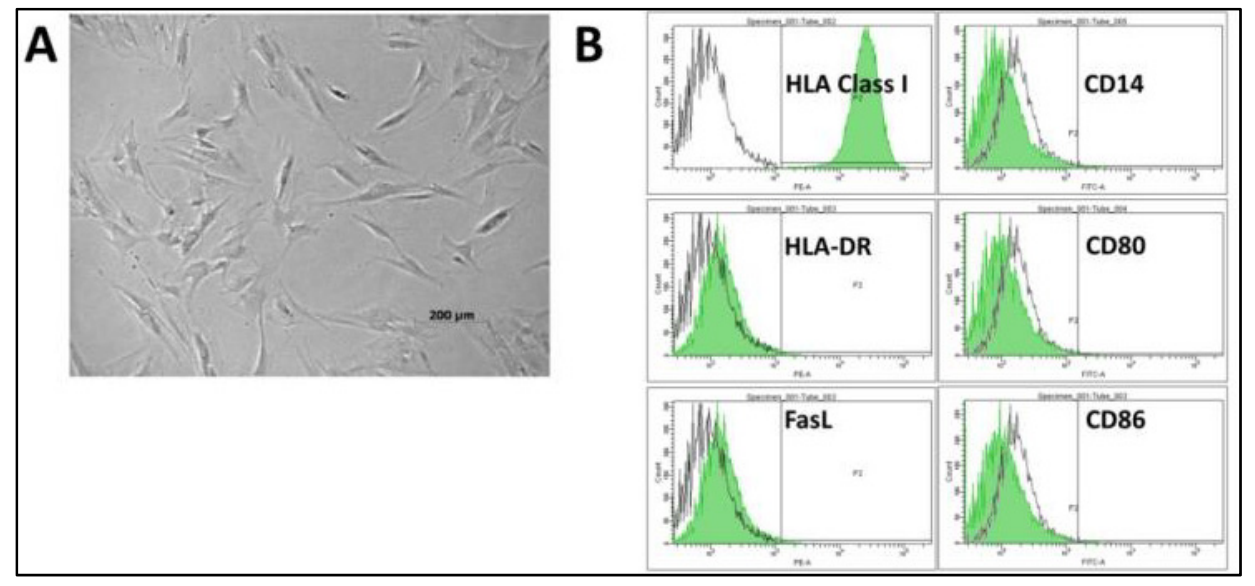

Figure 6-2. Characterization of human BMSCs

A. BMSCs are spindle-shaped adult stem cells under bright field. B. BMSCs are positive for HLA class I and negative for HLA-DR, FasL, CD14, CD80 and CD86. Open circle represents the isotype control. 
fluorescence following each division. The co-incubation with BMSCs strongly inhibited the proliferation of PBMCs as indicated by the area of successive generations at $24 \mathrm{~h}$ after co-incubation (Figure 6-3A). The total number of PBMCs in the co-culture was also significantly less than the PBMCs cultured alone (Figure 6-3B). Moreover, less IFN- $\gamma$, IL-2 and IL-2sR $\alpha$ (a soluble subunit of IL-2 receptor) were detected in the co-culture, suggesting less $\mathrm{T}$ cell activation (Figure 6-3C). We then determined whether the inhibition required direct contact between PBMCs and BMSCs. Surprisingly, BMSCs were equally potent to inhibit the proliferation of PBMCs and the expression of IFN- $\gamma$, IL-2 and IL-2sR $\alpha$ in a transwell system, suggesting a predominant paracrine model. Or in other words, BMSCs inhibited PBMCs by secreting soluble factors.

IL-10 is a well-documented immunosuppressive factor by suppressing T cell activation, preventing antigen presenting process and inhibiting inflammatory cytokines. We previously reported marginal IL-10 expression $(\sim 20 \mathrm{pg} / \mathrm{mL})$ in the medium of primary BMSCs [52]. However, IL-10 expression was significantly boosted $(300 \sim 400 \mathrm{pg} / \mathrm{mL})$ in the PBMC/BMSC co-culture, despite the existence of PHA-stimulation (Figure 6-4A). The inhibition of IL-10 pathway partially restored the production of IFN- $\gamma$, IL-2 and IL$2 \mathrm{sR} \alpha$ by the stimulated T cells in the presence of BMSCs (Figure 6-4B), indicating the crucial role of IL-10 in the immunomodulatory effects of BMSCs. We next determined which subpopulation of PBMCs produced IL-10. Neither T cells nor B cells have been reported to produce potent IL-10. We found that CD14+ monocytes were required for IL10 expression in the PBMC/BMSC co-culture since CD14+ monocyte depleted PBMCs produced significantly less IL-10 (Figure 6-4C). Realtime PCR also demonstrated that IL-10 transcription was activated in CD14+ monocytes instead of T cells or B cells, suggesting that the signal transduction between BMSCs and monocytes might be the first step toward the production of IL-10 and subsequent immune suppression.

Other literatures showed positive expression of FasL on the surface of BMSCs and hypothesized that BMSCs promoted the apoptosis of $\mathrm{T}$ cells and subsequent immune tolerance $[58,146]$. However, we did not detect FasL expression on the surface of BMSCs maintained in our lab (Figure 6-1). Annexin V staining demonstrated that BMSCs had no effect on the apoptosis of T cells in the co-culture (data not shown). Our results was supported by the results from Ryan et al [55].

\section{BMSCs improved islet function}

Bedsides immune rejection, islets also suffers from the PNF which is the loss of islet function by non-immune reactions. Although still in debate, inflammatory cytokines seem to play important roles in the PNF of human islets because raised inflammatory cytokines was accompanied with most post-transplantation challenges including hypoxia in the core of islet grafts, inflammation in the transplantation site and the disruption of vasculature [17]. In this study, we used a cocktail of inflammatory cytokines (TNF $\alpha 50$ $\mu \mathrm{g} / \mathrm{mL}, \mathrm{IL}-1 \beta 5 \mu \mathrm{g} / \mathrm{mL}$ and IFN- $\gamma 50 \mu \mathrm{g} / \mathrm{mL}$ ) to mimic the PNF related challenges to human islets. Without inflammatory cytokines, the presence of BMSCs in the co-culture 


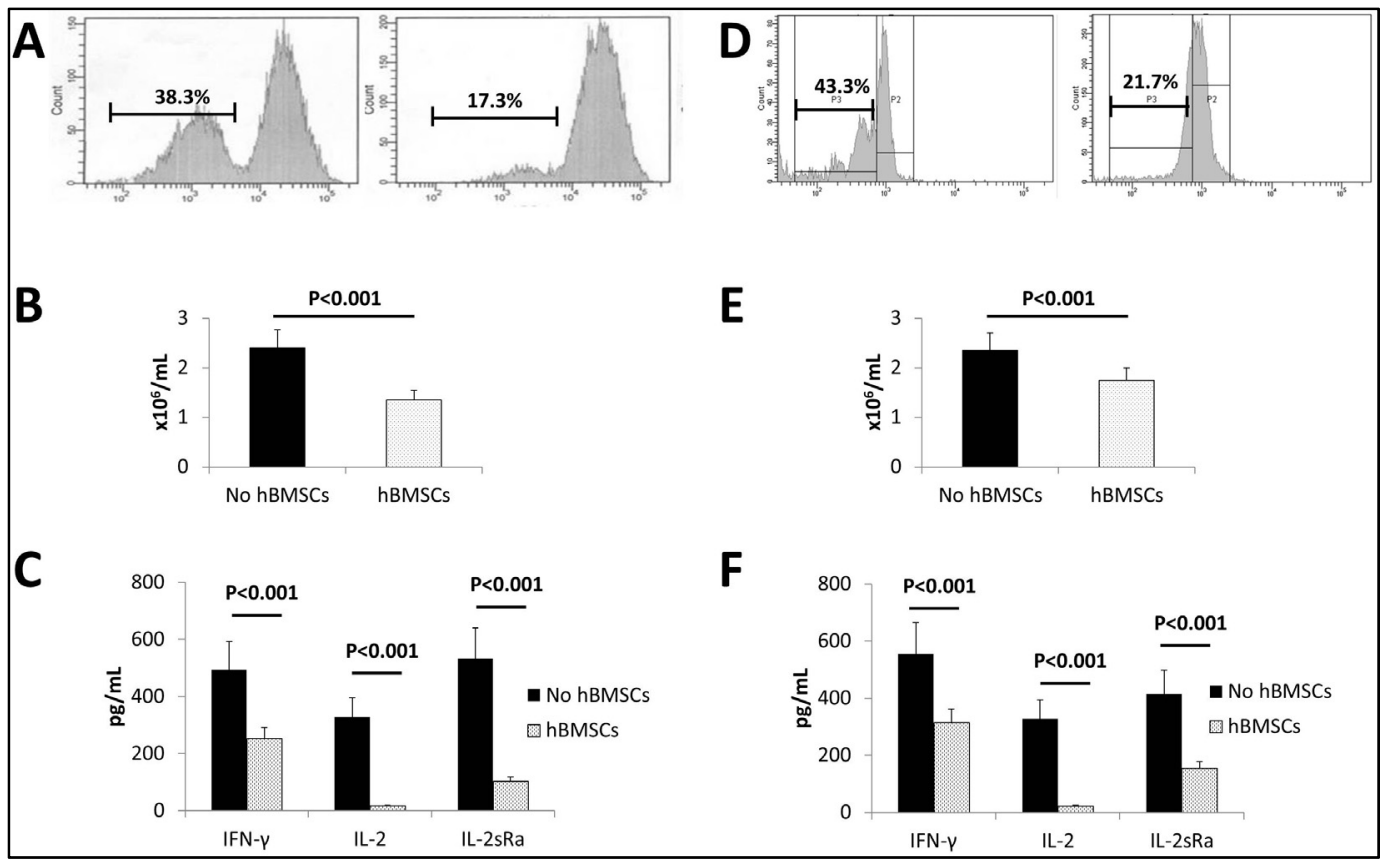

Figure 6-3. Human BMSCs prevent the proliferation and activation of PBMCs A. The percentage of successive generations of PBMCs when stimulated with phytohaemagglutinin (PHA, $1 \mu \mathrm{g} / \mathrm{mL}$ ) for 1 day and allowed to proliferate for 2 more days. PBMCs were labeled with CFSE and subjected to analysis using flow cytometry. Left, PBMCs alone; right, PBMCs with BMSCs. B. The total amount of PBMCs as determined by an automatic cell counter. C. The levels of IL-2, IL-2sR $\alpha$ and IFN- $\gamma$ in the medium as determined by ELISA. D-F. Repeat of the previous experiments (a-c) using a transwell system. $\mathrm{n}=3$. 


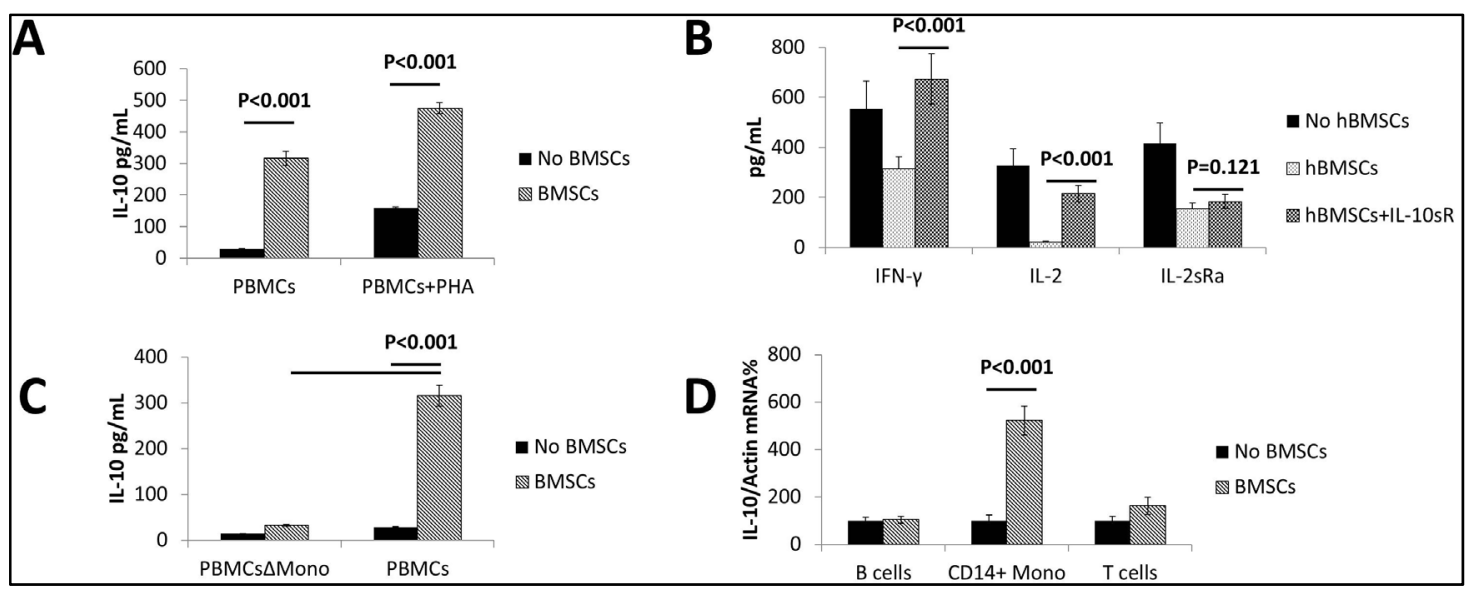

Figure 6-4. CD14+ monocytes and IL-10 were required for the immunomodulatory effect of BMSCs

PBMCs were stimulated with phytohaemagglutinin (PHA, $1 \mu \mathrm{g} / \mathrm{mL}$ ) for 1 day and cocultured with BMSCs for 2 more days. A. IL-10 was highly expressed by the PBMC/BMSC co-culture but not by PBMCs alone. B. The inhibition of PBMCs mediated by BMSCs can be reversed by adding IL-10sR. C. Monocyte depleted PBMCs $\left(\mathrm{PBMCs}^{\Delta \mathrm{Mono}}\right.$ ) failed to produce high level of IL-10 in the PBMC/BMSC co-culture. D. IL-10 mRNA was highly expressed in CD14+ monocytes but not B cells and T cells in the PBMC/BMSC co-culture. $\mathrm{n}=3$. 
did not affect insulin release from human islets when exposed to stimulatory glucose. Inflammatory cytokines significantly impaired the first-phase (the first peak appears immediately after stimulatory glucose) and the total insulin release of human islets after 4 days (Figure 6-5A). However, the presence of BMSCs in the co-culture significantly ameliorated the impairment caused by inflammatory cytokines (Figure 6-5B), suggesting a protective effect of BMSCs.

\section{BMSCs improved islet transplantation in humanized NSG mice}

The co-administration of STZ and PBMCs usually led to strong T cell alloreactivity and subsequent GvHD and death. In this study, we first used a long term injection of low doses of STZ to induce diabetes. Mice were mostly diabetic after three weeks. Then, 500 handpicked human islets were transplanted under the kidney capsule of each diabetic NSG mouse with BMSCs at serial ratios (islet: $\mathrm{BMSC}=1: 0,1: 50,1: 100$ and 1:200). At 4 weeks after successful islet transplantation, PBMCs $\left(5 \times 10^{6} /\right.$ mouse $)$ were injected intraperitoneally to establish the human immunity. This strategy helped us to identify the settlement of human immunity by comparing blood glucose before and after PBMC injection and to avoid STZ-stimulated GvHD. Glucose measurement showed that all mice restored normoglycemia during the first 4 weeks after islet transplantation, indicating successful islet engraftment and function (Figure 6-6A). However, the blood glucose of some mice reversed to hyperglycemia after PBMC infusion, probably due to the destruction of islet grafts by the established human immunity. Briefly, 11/11 mice receiving islets only were back to diabetes in the first two weeks after PBMC infusion. In contrast, 10/11,6/12 and 2/10 mice were back to diabetes when the islets were transplanted with BMSCs (islets: BMSCs=1:50, 1:100, 1:200), respectively (Figure 6-6B). Dose dependent improvement of the outcome of human islet transplantation was observed by increasing the ratio of BMSCs, suggesting the protective role of BMSCs against the adoptively transferred human immunity.

Intraperitoneal glucose tolerance test was performed 2 weeks after the PBMC injection to determine the function of islets in real time. Despite the difference in the nonfasted blood glucose level between different groups, all mice displayed similar baseline blood glucose levels following overnight fasting. Blood glucose levels increased immediately following glucose $(2 \mathrm{~g} / \mathrm{kg})$ injection, peaked at $15 \mathrm{~min}$ in all groups, and decreased over time (Figure 6-6C). The mice receiving islet/BMSC co-transplantation showed faster and better response to the stimulatory glucose compared to the mice receiving islets alone (Figure 6-6C), suggesting a better glucose control. At the end of study, the mice were anesthetized to collect blood to measure serum insulin and c-peptide level by ELISA. The levels of insulin and c-peptide of the mice receiving islet/BMSC cotransplantation were significantly higher than those of the mice transplanted with islets alone (Figure 6-6D). 


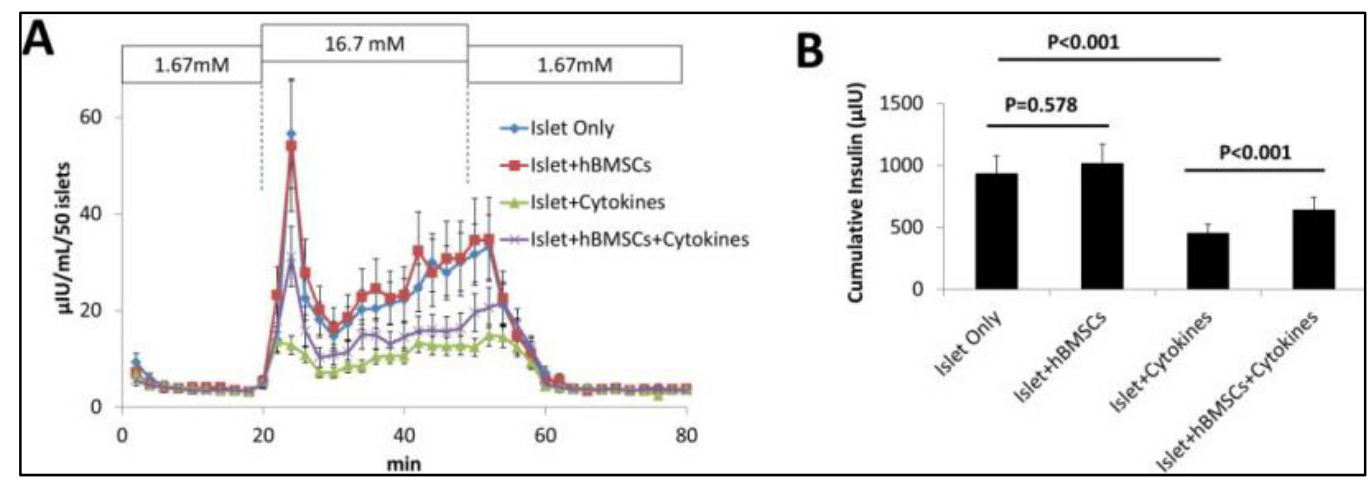

Figure 6-5. Human BMSCs protected the function of human islets against the inflammatory cytokines

A. The dynamic insulin release from human islets. Briefly, 50 islets from each group were perifused with basal glucose $(1.67 \mathrm{mM})$ for $60 \mathrm{~min}$, stimulatory glucose $(16.7 \mathrm{mM})$ for $30 \mathrm{~min}$ and finally basal glucose until the insulin release reversed to the basal level. The flow rate was maintained at $1 \mathrm{ml} / \mathrm{min}$ and the temperature was maintained at $37^{\circ} \mathrm{C}$. Samples were collected once per two minutes and analyzed for insulin concentration. For the cytokine treatment groups, islets, alone or with BMSCs, were stimulated with a cytokine cocktail (TNF $\alpha 50 \mu \mathrm{g} / \mathrm{mL}$, IL-1 $\beta 5 \mu \mathrm{g} / \mathrm{mL}$ and IFN- $\gamma 50 \mu \mathrm{g} / \mathrm{mL}$ ) for 4 days prior to perifusion. B. Cumulative insulin release calculated from dynamic insulin release profile on the left. $n=3$. 
Figure 6-6. The outcome of human islet transplantation with BMSCs in diabetic humanized NSG mice

A. The blood glucose levels of all mice after successful islet transplantation. For each mouse, 500 handpicked islets $((150 \sim 300 \mu \mathrm{m}$ in diameter $)$ were transplanted alone or with BMSCs at serial ratios (islet: $\mathrm{BMSC}=1: 50,1: 100$ and 1:200) under the kidney capsule of STZ induced diabetic NSG mice. The blood glucose level of each mouse was monitored using a glucometer weekly. An intraperitoneal injection of PBMCs $\left(5 \times 10^{6} / \mathrm{mouse}\right)$ was given 4 weeks after islet transplantation. At 17 weeks after islet transplantation, the graftbearing kidneys were then removed from some mice to validate the function of islet grafts by the return of blood glucose levels to $\geq 400 \mathrm{mg} / \mathrm{dL}$. B. The Kaplan-Meier plot for the percentage of insulin-independent mice of each group after PBMC injection. The mouse with blood glucose level $<200 \mathrm{mg} / \mathrm{dL}$ for two consecutive measurements was identified as insulin-independent. $\mathrm{P}$ value was calculated as compared to the islet-alone group using $\mathrm{R}$ version 2.15.2. C. Intraperitoneal glucose tolerance test was done with the mice receiving islet alone and islet/BMSC cotransplantation (islet: $\mathrm{BMSC}=1: 200$ ) to determine the islet function at 2 weeks after the injection of PBMCs. D. The serum insulin and c-peptide of the mice receiving islet alone and islet/BMSC cotransplantation (islet: $\mathrm{BMSC}=1: 200$ ) at the end of study. Data are presented as Mean $\pm \mathrm{SD}, \mathrm{n}=9$. 


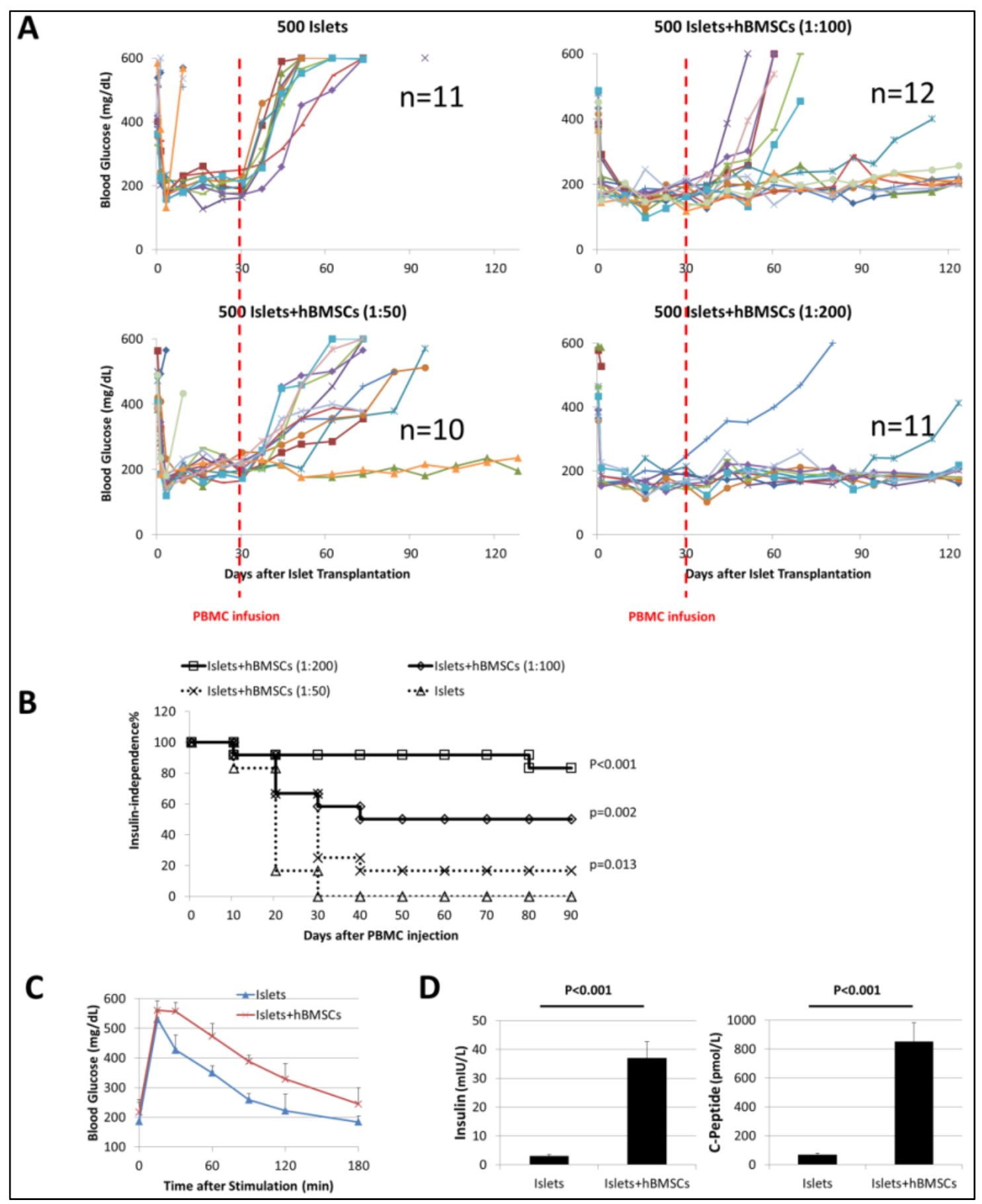




\section{BMSCs prevented $T$ cell infiltration and increased Tregs}

The kidney bearing islets was isolated at the end of study and subjected to immunofluorescence staining. The location and function of islet grafts were identified by staining for insulin. Insulin signal in the kidney capsule bearing islet/BMSC was significantly higher than those bearing islets only (Figure 6-7A). However, CD3+ T cells were significantly less in the kidney capsules bearing islet/BMSC than those bearing islets only. Taken together, these data suggested that BMSCs prevented $\mathrm{T}$ cell infiltration into the transplantation site and prevented the destruction of islets in vivo.

The expansion of Tregs might play an important role in the immune tolerance of transplanted organs in vivo [148]. Moreover, BMSCs were recently reported to increase the proportion of Tregs $[149,150]$. To further explore whether such mechanism can explain the reduced infiltration and destruction by alloreactive $\mathrm{T}$ cells into the transplantation site, we isolated all CD3 + T cells and detected the percentage of Tregs from the blood stream of NSG mice. Despite the low cell recovery, significantly higher percentage of $\mathrm{CD} 3+\mathrm{CD} 25+\mathrm{Foxp} 3+$ Tregs was detected in the blood stream (Figure 6-7B), which was consistent with the BMSC/T cells co-culture study in vitro (Figure 6-7C). Taken together, these results suggested that BMSC mediated increase in the proportion of Tregs might contribute to the immune tolerance of islet transplantation.

\section{Discussion}

Edmonton protocol for human islet transplantation, which was developed more than 20 year ago, failed to maintain insulin independence in the long term in most patients. Despite the development of new immunosuppressive regimens and new combination therapy, graft rejection remains a major challenge for human islet transplantation. Other challenges are summarized as PNF caused by the hypoxic environment, secretion of inflammatory cytokines and apoptosis of islet cells during isolation, transportation and storage process.

MSC-based cell therapy has attracted wide attention in regenerative medicine and tissue engineering. There are two major strategies of using MSCs for islet transplantation: 1) direct the differentiation of MSCs to replenish the lost islets; 2) use MSCs to nourish islet grafts and mitigate immune response. For the first strategy, several groups have reported the generation of islet-producing cells and islet-like clusters by the in vitro transdifferentiation of MSCs $[76,77]$. However, this strategy usually required long-term in vitro induction and failed to generate enough islets for clinical study and the risk of tumorigenicity was somehow overlooked. For the second strategy, MSCs constitutively secreted multiple factors, which are capable of regulating insulin production, revascularization, immune rejection and apoptosis of human islets. This strategy does not require in vitro transdifferentiation and huge amount of MSCs. Most importantly, MSCs can be isolated from and infused back into the same patient to exert the therapeutic potential as indicated by several clinical studies [39]. Therefore, in this study, we selected the second strategy for improving human islet transplantation. 


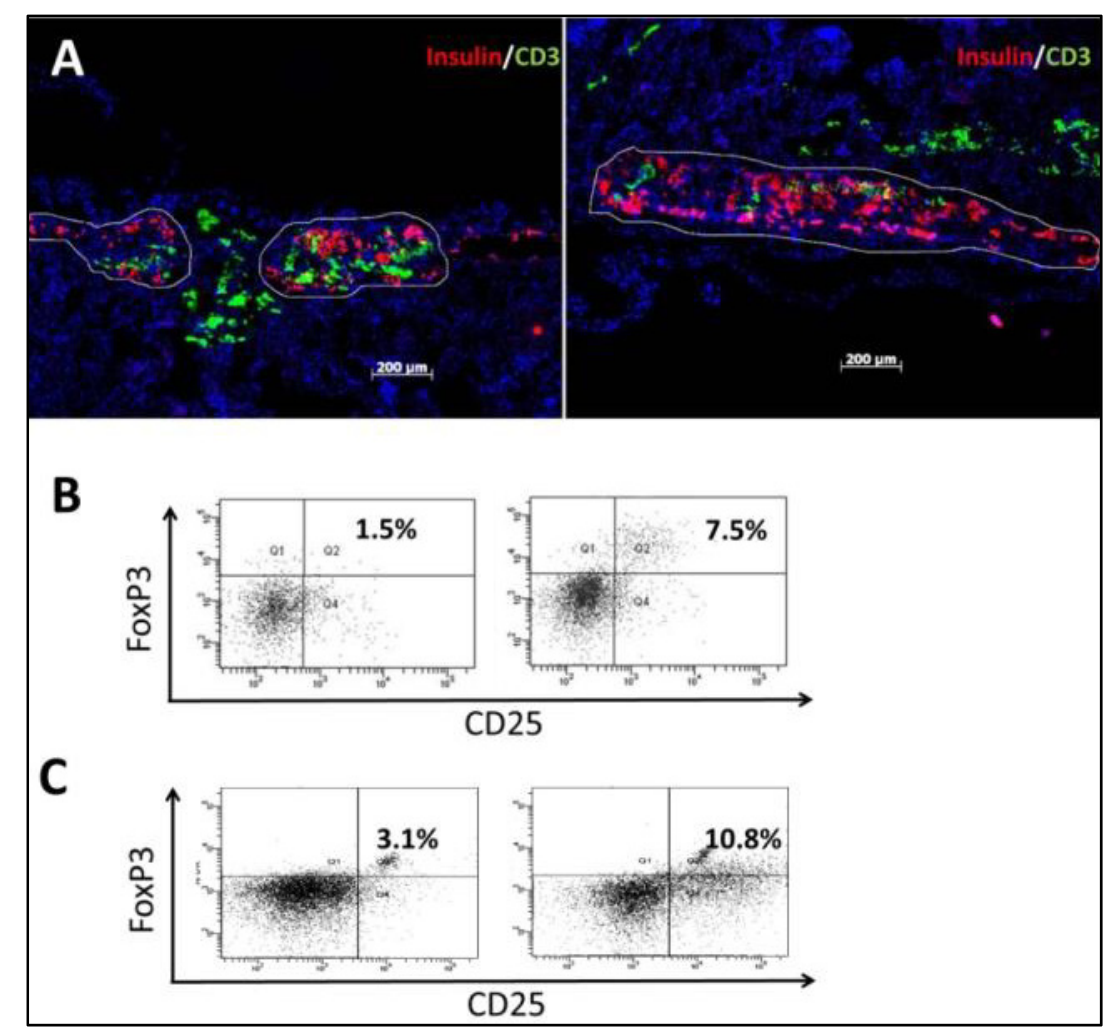

Figure 6-7. Human BMSCs prevent the $T$ cell infiltration into the transplantation site

A. The immune fluorescence staining of the kidney section bearing human islets alone (left) or with BMSCs (right). Briefly, three mice in each group were sacrificed at 2 weeks after the injection of PBMCs $\left(5 \times 10^{6} /\right.$ mice), or 6 weeks after islet transplantation. Insulin was stained in red to identify the transplanted human islets, while CD3+ T cells were stained in green. All experiments were done in triplicates and an representative picture was shown. B. The percentage of $\mathrm{CD} 4+\mathrm{CD} 25+\mathrm{FoxP} 3+$ regulatory $\mathrm{T}$ cells (Tregs) recovered from the blood stream of NSG mice at 2 weeks after the injection of PBMCs. Briefly, the total CD4+ T cells was isolated and purified from the total blood of NSG mice using Dynalbeads at the end of study. The percentage of CD25+FoxP3+ Tregs among the total CD4+ T cells was determined by flow cytometry. Q2 represents the Tregs. C. The percentage of Tregs of the total CD3 $+\mathrm{T}$ cells when co-culture with BMSCs in vitro. 
The pattern of the surface markers has great impact on the function of BMSCs and provided useful information to the following studies. Our BMSCs were positive for HLA class I and negative for CD14 (Figure 6-2), which concurred with previous report and suggested BMSCs can be recognized and destroyed by the cytotoxic T cells [55]. BMSCs were also negative for costimulatory factors such as CD80 and CD86, which might explain why BMSCs were unable to stimulate the multiplication of helper $\mathrm{T}$ (Th) cells and the subsequent antibody production. Generally, the lack of co-stimulatory factors (CD80 and CD86) led to an immature antigen presenting to the Th cells and subsequent immune tolerance [151]. Despite that some groups showed that BMSCs expressed FasL and promoted the apoptosis of T cells through direct contact [146, 152], our BMSCs were negative for FasL (Figure 6-2), which was supported by the results from other groups $[55,153]$ and confirmed by the MLR that BMSCs did not promote the apoptosis of T cells (data not shown). The expression of HLA class II on the surface of BMSCs is still unclear. While some groups demonstrated that BMSCs were positive for HLA class II [151], the studies from our and other groups suggested that BMSCs were negative for HLA class II which explained the hypoimmunogenicity of MSCs (Figure 6-2) [55]. This discrepancy is probably due to the difference in culture conditions and the sources of BMSCs and can only be solved if a standard operating protocol is developed and maintained among all research groups.

T cells are the most important cells mediating the recognition and destruction of human islets. T cells can be activated by PHA and the activation of T cells is characterized by the production of IL-2, IL-2R and IFN $\gamma$. In the MLR, BMSCs prevented PHA-induced proliferation and activation of PBMCs by secreting the soluble factors, among which IL-10 may play an important role (Figures 6-3, 6-4). However, despite potent IL-10 expression in the PBMC/BMSC co-culture, significantly less IL-10 was expressed by BMSCs or PBMCs when they were cultured alone [52], suggesting that the communications between BMSCs and a subpopulation of PBMCs were required for the production of immunosuppressive IL-10 $[63,65]$. We identified CD14+ monocytes as the prerequisite of the BMSC-mediated immune suppression (Figure 6-4), which is consistent with previous reports that monocytes or monocyte-derived dendritic cells were deeply involved in the BMSC-mediated T cell inhibition [154, 155]. First, BMSCs secreted soluble factor(s) and activated CD14+ monocytes to express IL-10. Then IL-10 suppressed the subsequent proliferation and activation of T cells. Such two-step immunosuppression could be an important feature for the co-transplantation of human islets and BMSCs as the BMSCs only exert a local effect to protect human islets without impairing the host immunity systemically. However, these results should be interpreted with caution because 1) the role of IL-10 in the BMSC-mediated immunosuppression is controversial as some groups observed IL-10 inhibition did not reverse and even further suppressed $\mathrm{T}$ cell proliferation $[63,154]$ and 2) the soluble factors secreted by BMSCs in this process remained unknown. Besides IL-10, other immunosuppressive factors such as HGF, TGF $\beta$ and PGE2 may also play important roles in the BMSC-mediated immunosuppression $[52,55,156]$. It is highly possible that the immunomodulatory effect of BMSCs is mediated through a network of molecular pathways instead of a single pathway. 
Next we evaluated the interactions of BMSCs with human islets and human immunity in vivo. In fact, several groups have previously shown the improvement of islet transplantation using MSCs, either through co-transplantation or intravenous infusion. For example, Ito et al. reported that MSCs improved the outcome of islet transplantation by promoting the revascularization of islet grafts [54]. Ding et al. reported MSCs prevented the rejection of allogeneic islets by matrix metalloproteinase-2 and -9 (MMP2 and MMP9) [60]. Yeung et al. reported that MSCs protected human islets from inflammatory cytokines [144]. However, the lack of human immunity in most of these studies caused limited understanding regarding the immune mediated graft loss. Yet the mechanism of BMSC-mediated protection to human islets in the presence of human immunity has not been thoroughly understood. Although xenogeneic human islet transplantation in immunocompetent rodents has been reported [142], the distinct differences in the immune system between rodent and human hindered the translation into a real clinical advance. The humanized mouse developed by Dr. Lenny Schultz is a "state of the art" animal model for research related to human immunity [79]. First, it is a perfect model to study the development of type 1 diabetes, which is an autoimmune disease. Second, the interaction between transplanted islets and the adoptively transferred human immunity provide useful information about immune rejection after human islet transplantation. The human immunity was established in NSG mice by injection of human PBMCs and validated by the presence of human IgG in the blood steam and CD3 + T cells in the mouse spleen (Figure 6-1). The adoptively transferred human immunity posed a challenge, which is similar to the clinical immune rejection, to the transplanted human islets. Compared with previous studies using immunodeficient animals $[52,54]$, the duration of insulin independence was significantly reduced in humanized NSG mice, as evidenced by the rapid loss of islet and T cell infiltration (Figures 6-6A, 6-7A). Co-transplantation with BMSCs significantly prolonged the duration of insulin independence in the presence of human immunity (Figure 6-6A). The intraperitoneal glucose tolerance test and the serum levels of human insulin and c-peptide further validate the improved islet function in the islet/BMSC co-transplantation group (Figures 6-6C-D). The interactions between BMSCs and T cells may of critical importance to the protection to human islets. We previously demonstrated that BMSCs "switched on" the production of immunosuppressive IL-10 by CD14+ monocytes to prevent the proliferation and activation of T cells in co-culture (Figure 6-3). In vivo results showed that BMSCs prevented the infiltration of $\mathrm{CD} 3+\mathrm{T}$ cells into the transplantation site, probably due to the reversal to $T$ cell activation (Figure 6-7A). Besides, we also found increased immunosuppressive Tregs in the blood stream of humanized NSG mice receiving islet/BMSC co-transplantation, suggesting locally injected BMSCs still had the potential to exert a considerable systemic effect (Figure 6-7B). Similar results were found in the MLR and supported by the results from Weng et al. (Figure 6-7C) [150]. However, further explorations are still required to unveil the underlying pathways to the proliferation of Treg.

Besides the immune modulatory effect, BMSCs improved insulin production of human islets against inflammatory cytokines in an islet perifusion assay (Figure 6-5), suggesting the role of BMSC as a trophic mediator to prevent the PNF of human islets. BMSCs also produce multiple growth factors including HGF and VEGF to promote the 
revascularization, which has been explored in depth in immune deficient animal model by us and other groups $[52,54]$.

Based on our results, we hereby propose three possible mechanisms for BMSCmediated islet tolerance (Figure 6-8): 1) BMSCs inhibit alloreactive T cells by the immature antigen presenting and/or activating CD14+ monocytes to produce IL-10;2) BMSCs promote the proliferation of Tregs through soluble factors; 3) BMSCs prevent the PNF of islets. The protection of human islets by BMSCs in the presence of human immunity may be a result of the synergistic combination of all three mechanisms.

Despite the evidences that BMSCs significantly improved islet transplantation in humanized NSG mice, the data should be interpreted with caution as several issues needed to be resolved. First, we did not characterize the interactions of BMSCs with B cells and natural killer cells. Such characterization may uncover the new aspects of BMSCs mediated immune tolerance and is necessary in future. The second issue is the GvHD caused by the infused mature immunocytes. Most humanized NSG mice or even NRG-Akita mice, which simultaneously develop diabetes without STZ injection, will develop GvHD in 5 7 weeks after the infusion of PBMCs [79], providing us a limited window to evaluate the efficacy of BMSC cotransplantation. The amount of infused PBMCs has great effect on the onset of GvHD. The dose of $5 \times 10^{6}$ PBMCs was reported to be relatively safe to induce significant GvHD and adopted for this study [79]. Due to the presence of BMSCs which hold strong immunomodulatory effects we did not detect significant GvHD-like symptoms including weight loss, hunched posture, ruffled fur, reduced mobility and tachypnea in the mice receiving islet/BMSC cotransplantation. However, we did observe GvHD in the mice receiving islets alone from 5 weeks after the infusion of PBMCs. Humanized mice generated from hematopoietic stem cells may resolve the issue of GvHD [80]. The third issue is the route of the administration of BMSCs. Many reports have shown that the systemic administration of BMSCs prevented the onset of type 1 diabetes. However, the systemic injection of BMSCs may be sequestered in the lung and other sites. In this study, we chose islet/BMSC cotransplantation to exert a local effect, which might have more impact on the future clinical application because the immunity of the patients receiving islet transplantation will not be compromised. Previous studies from us and other groups also ruled out the possibility of BMSC migration and demonstrated that BMSC co-transplantation stayed in the proximity of islet grafts and helped the engraftment and revascularization of islet grafts $[52,54]$. Last but not the least, despite our demonstration that BMSCs from the third party is equally potent to protect human islets from adoptively transferred human immunity (Figure 6-7A). The mismatch in HLA typing remains a concern for future clinical application. To better fit the scope of personalized medicine, BMSCs and PBMCs isolated from the same subject would be preferred. That requires further collaboration of institutions with hospitals.

In summary, our studies demonstrated that co-transplantation with BMSCs can be a promising strategy to improve the current status of human islet transplantation. The BMSC served as a major role of immune modulator to protect islets from immune destruction while also as a trophic mediator to prevent the PNF of islet transplantation. 


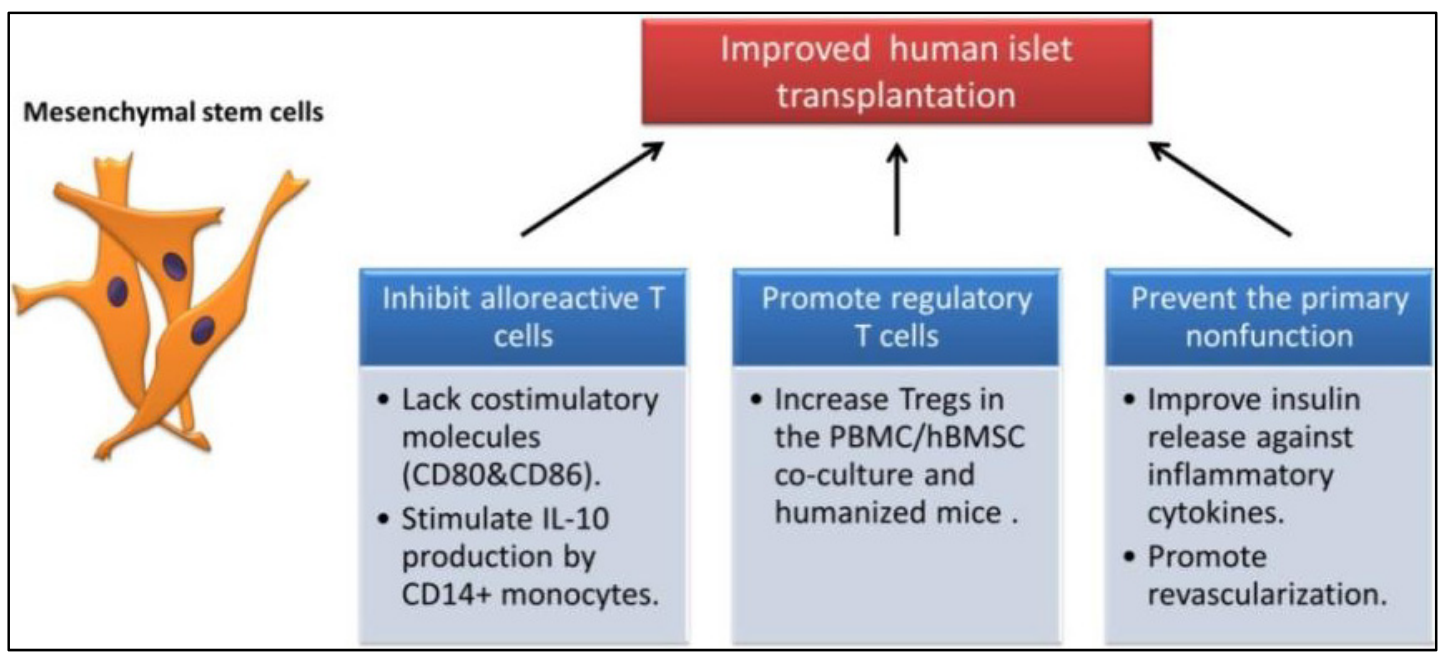

Figure 6-8. Schematic illustration of why BMSCs improved human islet transplantation 


\section{CHAPTER 7. A NOVEL COMPOUND DRUG FOR HUMAN ISLET TRANSPLANTATION IN HUMANIZED MOUSE MODEL}

\section{Introduction}

In the study described in Chapter 6 , we used BMSCs to prevent the immune rejection of human islet transplantation (Figures 6-6, 6-7). BMSCs also prevented the PNF of islet transplantation caused by inflammatory cytokines (Figure 6-5). Compared with gene therapy, stem cell therapy is a more biocompatible strategy for human islet transplantation. However, the unlimited proliferative capacity and transdifferentiation potential of stem cells especially genetically modified stem cells (Chapter 5) pose risks of tumorigenicity and immunogenicity, though in a lesser extent to islet recipient.

BMSCs infused intravenously will be sequestered in the lung. Therefore BMSCs were mostly co-transplanted with human islets and unsuitable for repeatedly injection, making the transplantation outcome hard to predict and manage. The cost and time needed for the isolation, expansion and even genetic modification of enough amounts of BMSCs for clinical application is another of concern using BMSCs. Therefore, in this study, we proposed a novel compound drug, in which an antiapoptotic drug is conjugated with immunosuppressive compound, for human islet transplantation. We hypothesized that the compound drug could prevent the inflammatory cytokine induced PNF and the immune rejection at the same time. Most importantly, this compound is easy to synthesize and can be injected repeatedly into a diabetic patient.

Zeng et al. synthesized a series of quinic acid (QA) derivatives and determined their anti-inflammatory activity in human A549 cells [157]. They demonstrated that among many QA derivatives, KZ41 had strongest inhibition of NK-kB activity against in A549 cells the stimulation of TNF $\alpha$ and can be used for as an anti-inflammation drug (Figure 7-1). We hypothesized that KZ41 was equally potent to suppress the inflammatory pathway in human islets and could prevent the PNF caused by inflammatory cytokines. MPA is an immunosuppressive drug frequently used in organ transplantation. MPA is commonly marketed under the trade names CellCept (Roche) and Myfortic (Novartis). MPA inhibits inosine monophosphate dehydrogenase, which controls the purine synthesis in T cells and B cells.

Therefore, in this study, we synthesized JP-3-110 by conjugating quinic acid amide and MPA via ester bond. We first compared the antiapoptotic effect of the compound drug JP-3-110 with its antiapoptotic counterpart KZ41 on human islets against inflammatory cytokines. We then compared the immunosuppressive effect of JP-3-110 with its immunosuppressive counterpart MPA against the activation and proliferation of $\mathrm{T}$ cells. Lastly, we evaluated the effectiveness of this new compound drug to improve the outcome of human islet transplantation in a diabetic humanized NSG mouse model. We hypothesized that the compound drug JP-3-110 can prevent the immune rejection and the PNF of human islet transplantation at the same time. 


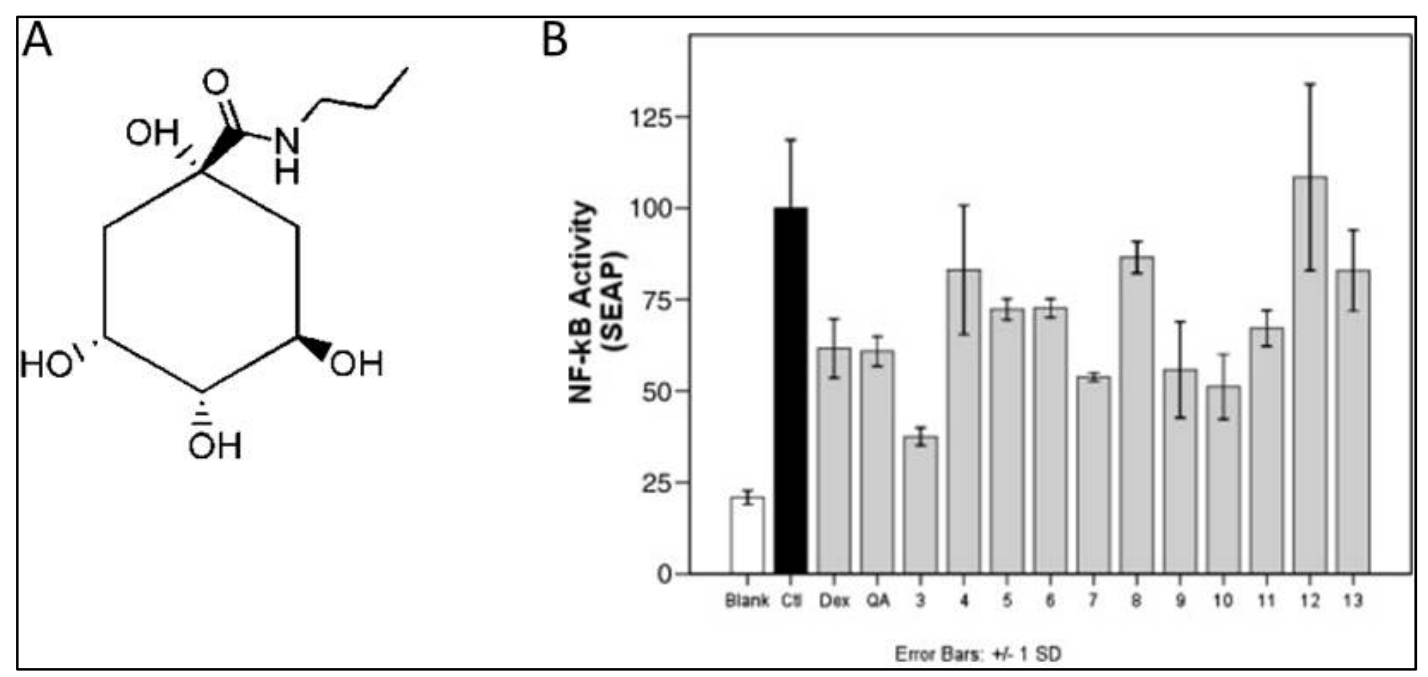

Figure 7-1. KZ41 and NF-kB inhibition

A. The structure of KZ41. B. NF-kB activity was measured using A549 cells stably transfected with a secreted alkaline phosphatase (SEAP) reporter at 18 hours after addition of TNF- $\alpha(10 \mathrm{ng} / \mathrm{mL})$ and either QA or QA derivatives $(3-13 ; 1 \mu \mathrm{M})$. KZ41 was lane 3. Dexamethasone (Dex; $1 \mu \mathrm{M}$ ) was used as a positive control. Data are presented as percent (\%) inhibition relative to control (ctl; TNF- $\alpha$ alone) and represent mean \pm SD. $n=$ 3. Blank: media only. 


\section{Materials and Methods}

\section{Materials}

Human islets were received from Integrated Islet Distribution Program (Duarte, CA). CMRL-1066 medium for islet culture and DAPI were purchased from Sigma Aldrich (St. Louis, MO). FBS was purchased from MediaTech Cellgro. (Herndon, VA). PBS was purchased from GIBCO-BRL (Gaithersburg, MD). NF-kB SEAPorterTM Assay Kit was purchased from IMGENEX (San Diego, CA). Human IL-2, IL-2sRa, IL-10 ELISA, TNF $\alpha$ and IFN- $\gamma$ ELISA kits were purchased from R\&D Systems (Minneapolis, $\mathrm{MN})$. The primary antibodies for CD3, CD4, insulin and the Dylight 488-conjugated secondary antibody were purchased from Abcam (Cambridge, MA). The Alexa Fluor 568 -conjugated secondary antibody and $0.25 \%$ trypsin were purchased from Invitrogen (Carlsbad, CA). Ultrasensitive One Touch glucose test strips and One Touch Ultra glucometer were purchased from LifeScan (Milpitas, CA). Tissue-Tek O.C.T. compounds were purchased from Sakura Finetek (Torrance, CA).

\section{Synthesis and characterization of antiapoptotic immunosuppressive drug}

All reagents for the synthesis were purchased from commercial sources and were used without further purification. Moisture-sensitive reactions were carried out under an argon atmosphere. Routine TLC was performed on aluminum backed Uniplates (Analtech, Newark, DE). NMR spectra were obtained on a Bruker ARX-400 MHz (Billerica, MA) or a Varian Inova-500 MHz spectrometer (Varian NMR Inc., Palo Alto, CA). Chemical shifts are reported as parts per million (ppm) relative to TMS $(0 \mathrm{ppm})$ in $\mathrm{CDCl}$. Temperature was regulated with a general accuracy of $\pm 0.1^{\circ} \mathrm{C}$. Mass spectral data were collected on a Bruker ESQUIRE-LC/MS system equipped with an ESI source. The synthesis of ester 4 used in the pancreatic islets protection studies was synthesized as illustrated in Figure 7-2. The starting compound 5,7-dihydroxy-2,2-dimethyl-N-propylhexahydrobenzo[d][1,3]dioxole-5-carboxamide (amide 1), which has been described before[158], was coupled with acetylated MPA 2 to obtain quinic acid ester 3 and further deprotection of acetanoid group using $1 \mathrm{~N} \mathrm{HCl}$ in tetrahydrofuran (THF) to give compound 4.

Synthesis of Compound 2. Acetic anhydride $(0.2 \mathrm{~mL}, 1.9 \mathrm{mmol})$ was slowly added to a pyridine $(5 \mathrm{~mL})$ solution of MPA $(0.2 \mathrm{~g}, 0.62 \mathrm{mmol})$ and $4-$ Dimethylaminopyridine (DMAP, $0.03 \mathrm{~g}, 0.25 \mathrm{mmol}$ ) at $0{ }^{\circ} \mathrm{C}$. The reaction mixture was stirred for $2 \mathrm{~h}$ and then poured onto crushed ice. The aqueous phase was acidified with 2 $\mathrm{M}$ aq. $\mathrm{HCl}(\mathrm{pH} \sim 2)$ and extracted with EtOAc $(3 \times 400 \mathrm{~mL})$. The combined organic extracts were dried over $\mathrm{Na} 2 \mathrm{SO} 4$, filtered, and the solvent was removed under reduced pressure to afford acetylated MPA $2(0.18 \mathrm{~g}, 83 \%)$ as a white powder mp $155-157^{\circ} \mathrm{C}$.

1H NMR (500MHz, $\left.\mathrm{CDCl}_{3}\right): \delta 5.18-5.5(\mathrm{~m}, 3 \mathrm{H}), 3.79(\mathrm{~s}, 3 \mathrm{H}), 3.28(\mathrm{~d}, \mathrm{~J}=6.9 \mathrm{~Hz}$, $2 \mathrm{H}), 2.43-2.38(\mathrm{~m}, 5 \mathrm{H}), 2.33-2.28(\mathrm{~m}, 2 \mathrm{H}), 2.21(\mathrm{~s}, 3 \mathrm{H}), 1.79(\mathrm{~s}, 3 \mathrm{H})$. Mass: 385.2 


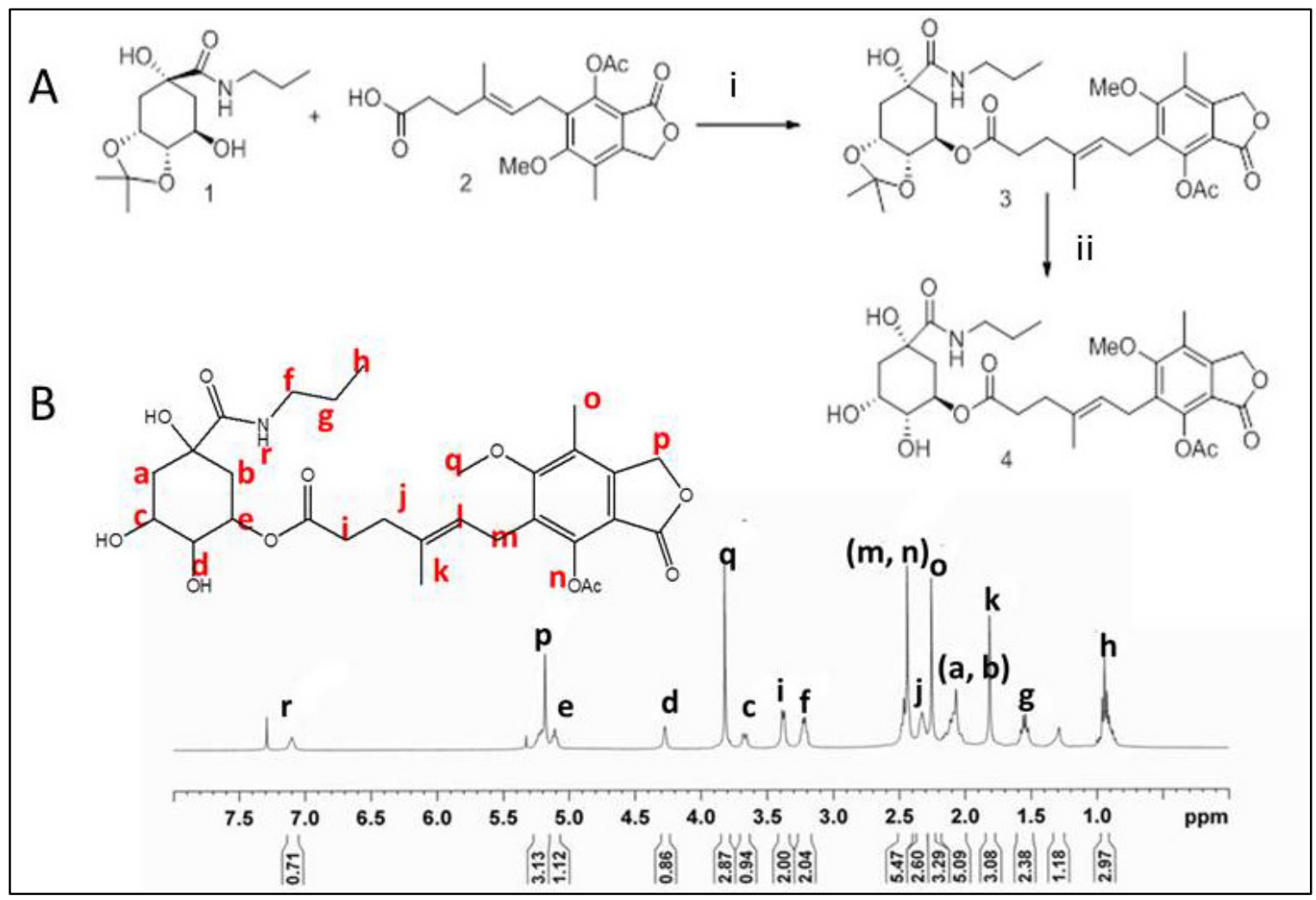

Figure 7-2. Synthesis and characterization of JP-3-110

A. The synthetic scheme of JP-3-110. i) DIC, DMAP, DCM, 73\%; ii) IN HCl/THF, 12h, $21 \%$. B. The $1 \mathrm{H}$ NMR spectrum of JP-3-110. 
$(\mathrm{M}+\mathrm{Na})$.

Synthesis of Compound 3. A solution of amide $1(0.32 \mathrm{~g}, 0.88 \mathrm{mmol})$, acetylated MPA $2(0.24 \mathrm{~g}, 0.88 \mathrm{mmol}), \mathrm{N}, \mathrm{N}$-dimethyl amino pyridine $(0.16 \mathrm{~g}, 1.32 \mathrm{mmol})$ and diisopropyl carbodiimide $(0.21 \mathrm{~mL}, 1.32 \mathrm{mmol})$ in $\mathrm{CH}_{2} \mathrm{Cl}_{2}(10 \mathrm{~mL})$ was stirred at room temperature under $\mathrm{Ar}$ atmosphere overnight. The reaction mixture was diluted with $\mathrm{CHCl} 3(20 \mathrm{~mL})$ and then washed with $1 \mathrm{~N} \mathrm{HCl}(10 \mathrm{~mL})$, water $(10 \mathrm{~mL})$, aqueous saturated $\mathrm{NaHCO} 3$ solution $(10 \mathrm{~mL})$, and brine $(10 \mathrm{~mL})$. The organic layer was dried (Na2SO4) and evaporated, and the residue was purified by column chromatography (silica gel, 30\% acetone in petroleum ether) to afford (E)-6-hydroxy-2,2-dimethyl-6(propylcarbamoyl)-hexahydrobenzo[d][1,3]dioxol-4-yl-6-(4-acetoxy-6-methoxy-7methyl-3-oxo-1,3-dihydroisobenzofuran-5-yl)-4-methylhex-4-enoate (compound 3, $0.4 \mathrm{~g}$, $73 \%)$ as a white solid $\mathrm{mp} 71-73^{\circ} \mathrm{C}$.

1H NMR (400MHz, $\left.\mathrm{CDCl}_{3}\right): \delta 7.08(\mathrm{t}, 1 \mathrm{H}), 5.32-5.23(\mathrm{~m}, 1 \mathrm{H}), 5.17(\mathrm{~s}, 1 \mathrm{H})$, 5.15-5.08 (m, 1H), 5.32-5.24 (m, 1H), 4.54-.48 (m, 1H), 4.14-4.08 ( m, 1H), $3.84(\mathrm{~s}, 1 \mathrm{H})$, $3.78(\mathrm{~s}, 3 \mathrm{H}), 3.5(\mathrm{~d}, \mathrm{~J}=6.9 \mathrm{~Hz}, 2 \mathrm{H}), 3.24-3.15(\mathrm{~m}, 2 \mathrm{H}), 2.45-2.36(\mathrm{~m}, 5 \mathrm{H}), 2.33-2.28$ $(\mathrm{m}, 2 \mathrm{H}), 2.23(\mathrm{~s}, 3 \mathrm{H}), 2.03-1.90(\mathrm{~m}, 2 \mathrm{H}), 1.76(\mathrm{~s}, 3 \mathrm{H}), 1.58(\mathrm{~s}, 3 \mathrm{H}), 1.55-1.48(\mathrm{~m}, 2 \mathrm{H})$, $1.36(\mathrm{~s}, 3 \mathrm{H}) .0 .93(\mathrm{t}, 3 \mathrm{H})$. Mass: $640.3(\mathrm{M}+\mathrm{Na})$.

Synthesis of Compound 4. Compound $3(0.4 \mathrm{~g}, 0.64 \mathrm{mmol})$ was dissolved in THF $(3 \mathrm{~mL})$, then aqueous $1 \mathrm{~N} \mathrm{HCl}(5 \mathrm{~mL})$ was added at room temperature and the reaction mixture was stirred for $12 \mathrm{~h}$ and monitored by ESI-MS. After completion of reaction, solution was saturated with solid $\mathrm{NaCl}$ and aqueous phase was extracted with ethyl acetate $(3 \times 100 \mathrm{~mL})$. The organic layer was dried (Na2SO4) and evaporated, and the residue was purified by column chromatography (silica gel, $90 \%$ ethyl acetate in petroleum ether) to afford compound $4(0.08 \mathrm{~g}, 21 \%)$ as a white solid $\mathrm{mp} 74-76{ }^{\circ} \mathrm{C}$.

1H NMR (400MHz, $\left.\mathrm{CDCl}_{3}\right): \delta 7.08(\mathrm{t}, 1 \mathrm{H}), 5.22-5.16(\mathrm{~m}, 3 \mathrm{H}), 5.11-5.05(\mathrm{~m}$, $1 \mathrm{H}), 4.84(\mathrm{~s}, 1 \mathrm{H}), 4.29-4.24(\mathrm{~m}, 1 \mathrm{H}), 3.80(\mathrm{~s}, 3 \mathrm{H}), 3.68-3.61(\mathrm{~m}, 1 \mathrm{H}), 3.38(\mathrm{~d}, \mathrm{~J}=6.9 \mathrm{~Hz}$, $2 \mathrm{H}), 3.32-3.30(\mathrm{M}, 1 \mathrm{H}), 3.25-3.16(\mathrm{~m}, 3 \mathrm{H}), 2.45-2.36(\mathrm{~m}, 5 \mathrm{H}), 2.33-2.28(\mathrm{~m}, 2 \mathrm{H}), 2.23$ $(\mathrm{s}, 3 \mathrm{H}), 2.15-2.0(\mathrm{~m}, 4 \mathrm{H}), 1.8(\mathrm{~s}, 3 \mathrm{H}), 1.51-1.50(\mathrm{~m}, 2 \mathrm{H}), 0.9(\mathrm{t}, 3 \mathrm{H})$. Mass: 578.3 $(\mathrm{M}+\mathrm{H})$.

\section{NF-kB activity}

INS-1E cells which were stably transfected with the SEAP gene containing the response-element for NF-kB were used to screen for anti-inflammatory activity. Briefly, 106 cells/well were seeded overnight followed by treatment with $10 \mathrm{ng} / \mathrm{mL}$ human recombinant TNF $\alpha$ and either KZ41 or hybrid drug JP-3-110 $(1 \mu \mathrm{M})$. SEAP activity was measured $18 \mathrm{~h}$ later in supernatant samples $(50 \mathrm{lL})$ using the NF-kB SEAPorterTM Assay Kit (IMGENEX) and a microplate luminometer. SEAP activity was normalized to the total protein content. Inhibitory potency (IC50) was determined from dose-response curves ( $\mathrm{n}=3$ separate experiments). 


\section{Caspase assay}

Caspase-Glo 3 assay kits were used to analyze caspase 3 as per the manufacturer's protocol (Promega). This assay kit provides a proilluminescent caspase substrate, DEVD, that, when cleaved by caspases, will release luciferin to quantitatively determine caspase concentration. Briefly, following sequential treatment by JP-3-110 for $48 \mathrm{~h}$ and a cytokine cocktail of recombinant TNF $\alpha(5 \mathrm{ng} / \mathrm{ml})$ and IL-1 $\beta(5 \mathrm{ng} / \mathrm{ml})$ for additional $48 \mathrm{~h}, 100 \mu \mathrm{L}$ of Caspase-Glo reagent was added to $100 \mu \mathrm{L}$ of culture supernatants in 96-well plates and incubated at room temperature for $1 \mathrm{~h}$. The contents were then transferred into culture tubes, and luminescence was determined using a luminometer (Berthold, Germany).

\section{Griess assay}

Nitric oxide (NO) production and accumulation is a vital step in the apoptotic pathway. Nitrite concentration was determined using the Griess assay (Promega, Madison, MI). Fifty microliters of cell culture supernatant was added to a 96 well-plate and mixed with $50 \mu \mathrm{L}$ of $1 \%$ sulfanilamide in $5 \%$ phosphoric acid solution and incubated for 5 min at room temperature in the dark. Then, $50 \mu \mathrm{L}$ of $0.1 \% \mathrm{~N}-1$ naphthylethylenediamine dihydrochloride (NED) aqueous solution was added to each well. The plate was incubated for an additional $10 \mathrm{~min}$, and absorbance was measured at $560 \mathrm{~nm}$ using a microplate reader. To determine the nitrite concentration in each sample, a standard curve was prepared using nitrite standard solution and blank culture.

\section{Apoptosis studies}

A cytokine cocktail was used to mimic the in vivo challenge to the INS-1E cells and human islets by the inflammatory cytokines. Briefly, following JP-3-110 treatment for $48 \mathrm{~h}$ and cytokine cocktail treatment for additional $48 \mathrm{~h}$, INS-1E cells were characterized by the DeadEnd ${ }^{\mathrm{TM}}$ Colorimetric TUNEL system (Promega, Madison, WI, USA), in which fragmented DNA from apoptotic cells was labeled with biotinylated nucleotide and detected using hydrogen peroxide and diaminobenzidine. Following treatment with JP-3-110 and cytokines, human islets were digested with $0.25 \%$ trypsin/EDTA into a single-cell suspension, stained with the Annexin V-FITC Apoptosis Detection Kit (Abcam) and analyzed with flow cytometry. Annexin V binds to phosphatidylserine on the cell surface, which is a feature found in apoptotic cells. Fluorescent intensity was analyzed using CellQuest software (BD Bioscience, Franklin Lakes, NJ, USA). Three sets of independent transduction experiments were carried out for each assay.

\section{Islet perifusion study}

The insulin secretion from human islets was quantified using a dynamic islet perifusion assay. Briefly, 50 islets from each group were handpicked and loaded onto a 
Swinnex 13 chamber (Millipore, Burlington, MA, USA) and perifused with Krebs-Ringer bicarbonate HEPES buffer of the following composition (in $\mathrm{mM}$ ): $129 \mathrm{NaCl}, 5 \mathrm{NaHCO} 3$, $4.8 \mathrm{KCl}, 1.2 \mathrm{KH} 2 \mathrm{PO} 4,1.2 \mathrm{MgSO} 4,2.5 \mathrm{CaCl} 2$, and $10 \mathrm{HEPES}$ at $\mathrm{pH}$ 7.4. The flow rate was maintained at $1 \mathrm{ml} / \mathrm{min}$ with a peristaltic pump (Themo Fisher, Waltham, MA) and the temperature was maintained at $37^{\circ} \mathrm{C}$ with a solution heater (Warner Instruments, Hamden, CT). Islets were first perifused with basal glucose $(1.67 \mathrm{mM})$ for $60 \mathrm{~min}$ and stimulated with glucose $(16.7 \mathrm{mM})$ for $30 \mathrm{~min}$ and finally perifused with basal glucose $(1.67 \mathrm{mM})$ until insulin release reversed to the basal level. Samples were collected once per two minutes through an automatic fraction collector (Waters, Milford, MA) and analyzed for insulin content by ELISA.

\section{Mixed lymphocyte reaction (MLR)}

PBMCs were isolated using Ficoll Paque as described in Chapter 6. T cells were isolated from PBMCs using Dynabeads for. T cells were stimulated with PHA $(1 \mu \mathrm{g} / \mathrm{ml})$ for $24 \mathrm{~h}$ and then cultured in the presence of KZ41 $(5 \mu \mathrm{M})$ or JP-3-110 $(5 \mu \mathrm{M})$ or additional 4 days. The total number of PBMCs was measured using T4 Automatic Cell Counter (Nexcelom, Lawrence, MA). The extent of T cell activation was determined by measuring the level of IL-2, IL-2sRa, TNF $\alpha$ and IFN- $\gamma$ in the medium using ELISA at indicated time.

\section{Rebuild human immunity}

Human PBMCs were isolated from buffy coats by gradient centrifugation using Ficoll Paque. The T cells and B cells of PBMCs were stained with FITC conjugated CD3 antibody and APC conjugated CD19 antibody, respectively, and characterized using flow cytometry. The PBMCs were infused into diabetic NSG mice at the concentration of $5 \times 106 /$ mouse to establish human immunity. The settlement of $\mathrm{T}$ cells in the spleen was assessed by immunofluorescence staining. Briefly, spleen were isolated, washed with PBS, fixed in $4 \%$ paraformaldehyde overnight, and embedded in optimal cutting temperature compound. Frozen sections of $5 \mu \mathrm{m}$ thickness were cut. The slides were stained with rabbit anti-human CD3 primary antibody $(1: 200)$ at $4{ }^{\circ} \mathrm{C}$ overnight and Alexa Fluor 568 conjugated goat anti-rabbit secondary antibody (1:500) at room temperature for $1 \mathrm{~h}$. The function of B cells was assessed by measuring human $\operatorname{IgG}$ concentration in the mouse blood.

\section{Statistical analysis}

Statistical significance of the difference between the two groups was determined by unpaired t-test and between several groups by one-way ANOVA. 


\section{Results}

\section{Synthesis of JP-3-110}

Quinic acid amide analog KZ41 was synthesized as described before [157]. JP-3110 was synthesized by conjugating KZ41with MPA and the structure of JP-3-110 was confirmed by 1H NMR (Figure 7-2). The solubility of JP-3-110 was determined to be $0.48 \pm 0.16 \mathrm{mg} / \mathrm{ml}(0.80 \pm 0.27 \mathrm{mM})$ by HPLC, which is practically insoluble according to USP solubility criteria (Figure 7-3).

\section{Apoptotic effect of JP-3-110}

Although the water solubility of JP-3-110 was extremely poor, we still tried to determine whether JP-3-110 can suppress the NF-kB activity as its counterpart KZ41 in insulin producing INS-1E cells. A cocktail of inflammatory cytokines (TNF $\alpha 10 \mathrm{ng} / \mathrm{mL}$, IL-1 $\beta 5 \mathrm{ng} / \mathrm{mL}$ and IFN- $\gamma 10 \mathrm{ng} / \mathrm{mL}$ ) was used to mimic the inflammation in vivo. Results indicated that JP-3-110 was equally potent as KZ-41 to suppress the NF-kB activity of INS-1E cells under the stimulation of inflammatory cytokines (Figure 7-4). We then determined the activities of caspase 3 which is a converging point of apoptotic pathway. Results showed that INS-1E cells pre-treated with KZ-41 and JP-3-110 showed significant decrease in caspase 3 activity (Figure 7-5A), suggesting the inhibition of apoptotic pathway by KZ41 and JP-3-110. As a result, the inflammatory-cytokineinduced apoptotic cell death was significantly reduced in INS-1E cells in the presence of KZ41 or JP-3-110 (Figure 7-5B).

We then determine the protective effect of KZ41 on human islets against inflammatory cytokines. We demonstrated that the percentage of apoptotic cells in human islets were significantly reduced in the presence of KZ41 at 2 days and 4 days after stimulation with cytokine cocktail, suggesting a protective role of KZ41 (Figure 7-6). Interestingly, we also demonstrated that the INS-1E cells and human islets conditioned with KZ41 and JP-3-110 acquired similar resistance to the challenges of inflammatory cytokines (data not shown), suggesting that KZ41 and JP-3-110 can be used as preventive medicine.

\section{Immunosuppressive effect of JP-3-110}

Besides the PNF of islet grafts caused by inflammatory cytokines, immune rejection is another key barrier for successful islet transplantation. We determined whether JP-3-110 retained the immunosuppressive effect as MPA. Typically, T cell activation is characterized by an early and transient expression of IL-2 and a long-lasting expression of TNF $\alpha$ and IFN $\gamma$ (Figure 7-7). JP-3-110 showed minimal effect on the expression of IL-2 but it strongly inhibited the expressions of TNF $\alpha$ and IFN $\gamma$ in long term. Further study illustrated that JP-3-110 significantly suppressed T cell proliferation 


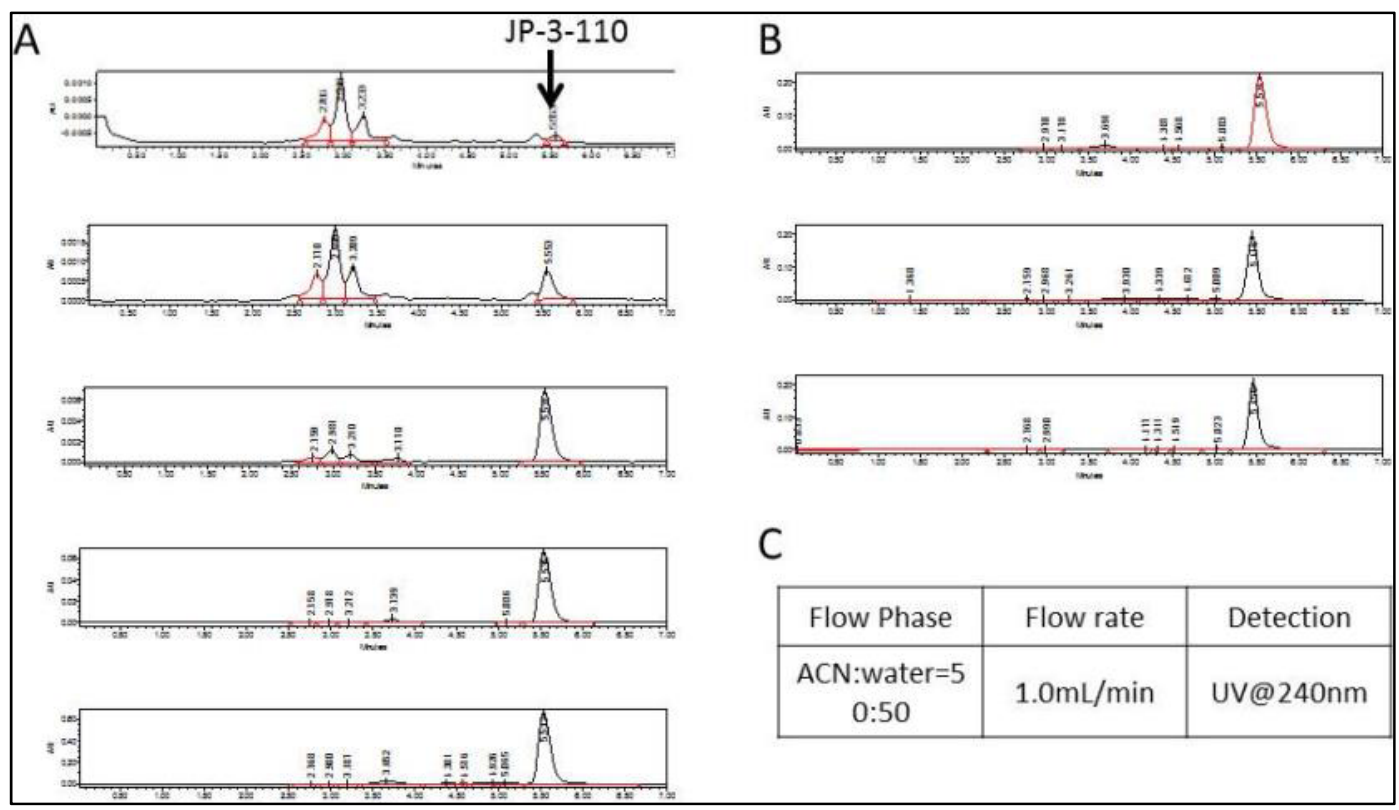

Figure 7-3. Use HPLC to determine the solubility of JP-3-110

A. Standard curve of JP-3-110. From top to bottom, 0.2, 2, 20, 200, $2000 \mathrm{mg} / \mathrm{mL}$ of JP-3110 in acetonitrile (ACN). B. Triplicates of JP-3-110 saturated water solution. C. HPLC conditions. Column: Inertsil ODS-3, 250 x 4.6mm, $5 \mu \mathrm{m}$. 


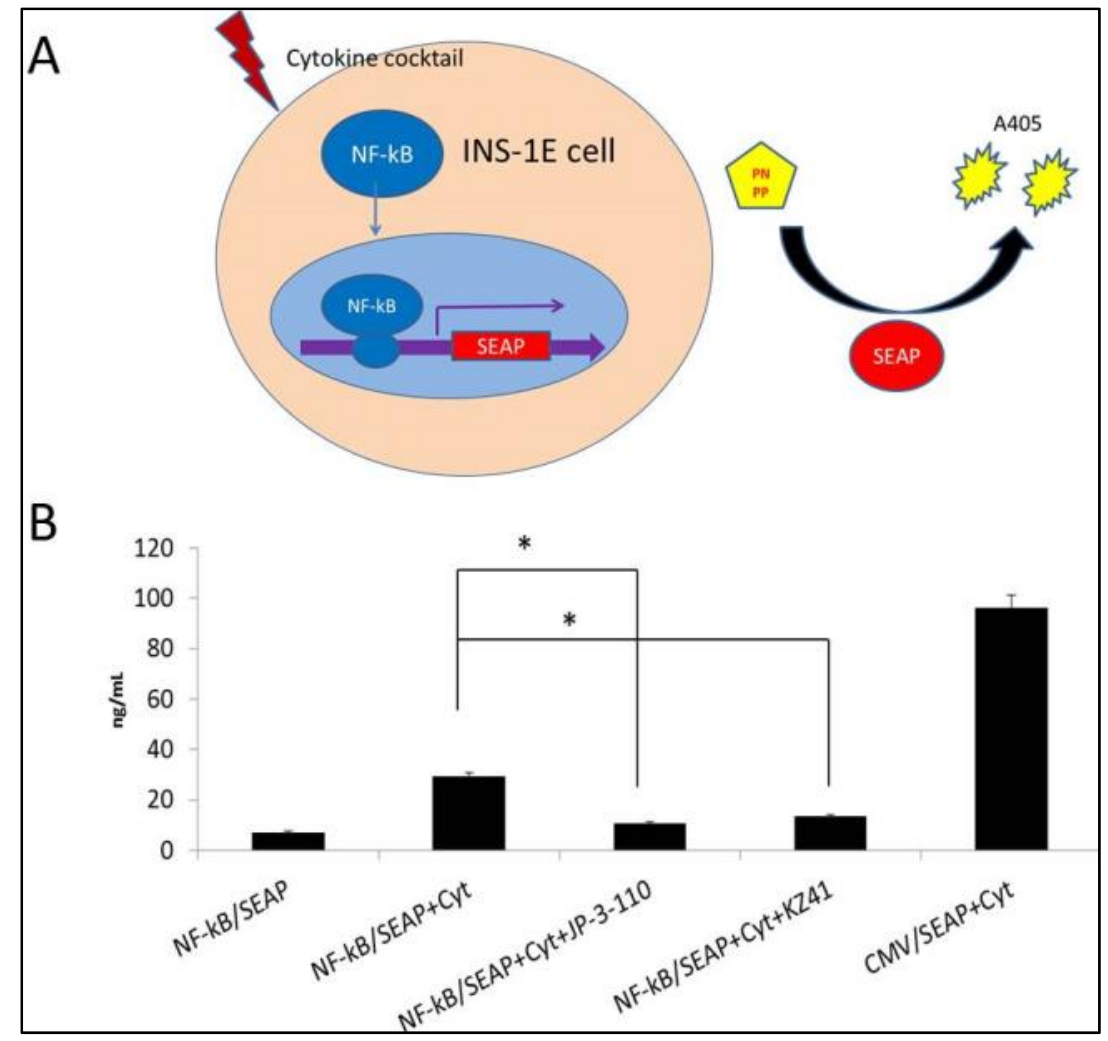

Figure 7-4. JP-3-110 and KZ41 suppressed NF-kB activity in INS-1E cells A. Schematic illustration of the mechanism of SEAP reported gene assay to determine NF-kB activity. Briefly, a plasmid construct containing NF-kB promoter and SEAP gene was transferred into INS-1E cells by lipofectamine. SEAP was then expressed and secreted into culture supernatant and allowed chemiluminescent detection using a substrate PNPP under $405 \mathrm{~nm}$. B. NF-kB activity was measured 24 hours after addition of cytokine cocktail and either JP-3-110 $(5 \mu \mathrm{M})$ or KZ41 $(5 \mu \mathrm{M})$. CMV/SEAP construct constitutively expressed SEAP and served as positive control. 


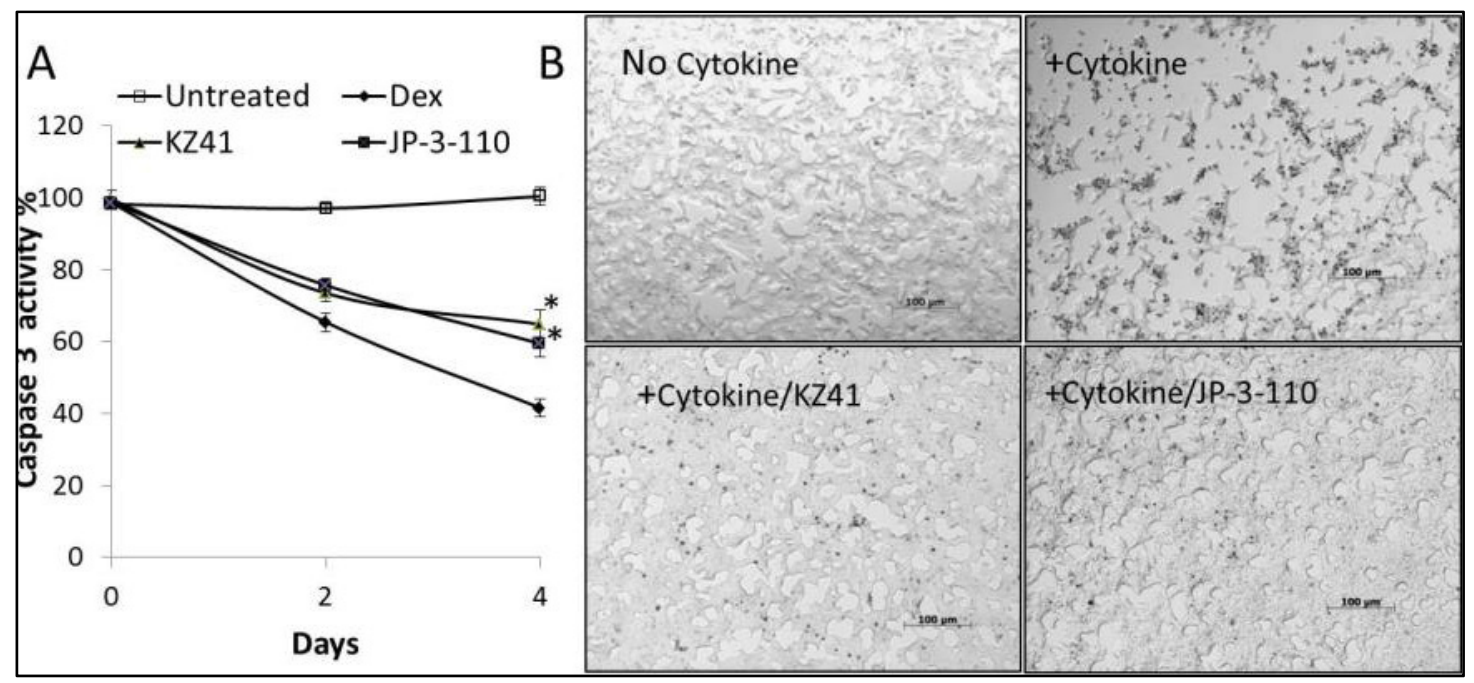

Figure 7-5. KZ41 and JP-3-110 pretreated INS-1E cells were resistant to cytokine induced apoptotic cell death

A. Caspase 3 activities in INS-1E cells after treated with KZ41 $(5 \mu \mathrm{M})$ and JP-3-110 $(5 \mu \mathrm{M})$. Dexamethasone $($ Dex, $1 \mu \mathrm{M})$ was used as a positive control. B. INS-1E cells were stimulated with a cytokine cocktail alone or in the presence of KZ41 or JP-3-110 for 2 day. Then all cells were subjected to TUNEL assay using DeadEnd Colorimetric TUNEL system. Apoptotic cells were stained in dark. ${ }^{*} \mathrm{p}<0.05$ as determined by an unpaired Student's t-test, $\mathrm{n}=6$. 


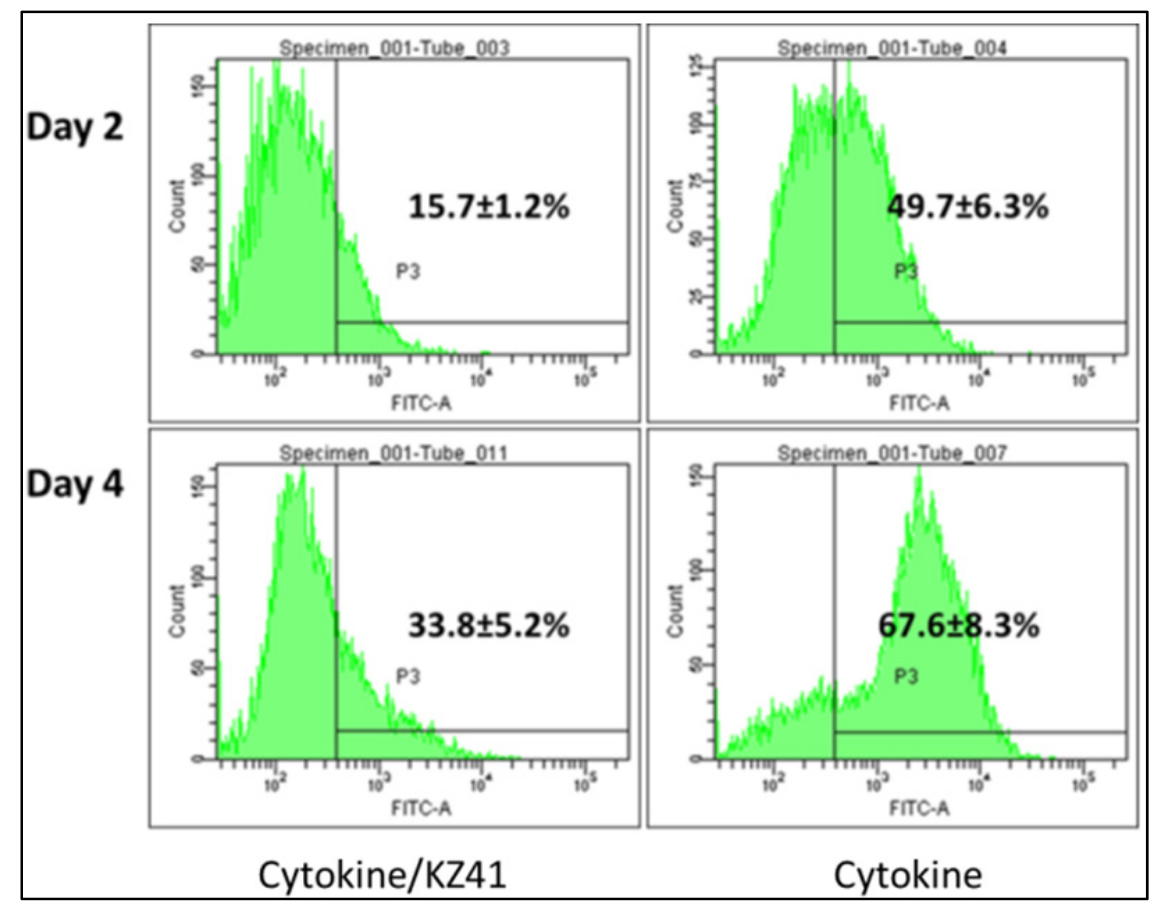

Figure 7-6. KZ41 inhibited the apoptotic cell death of human islets after stimulation with cytokine cocktail

Briefly, after stimulated with cytokine cocktail in the presence or absence of KZ41 $(5 \mu \mathrm{M})$ for indicated time, islet was collected and dispersed with $0.25 \%$ Trypsin/EDTA into single cell suspension. Apoptotic cells were stained with FITC labeled annexin V and counted by flow cytometry. P3 indicated the percentage of apoptotic cells. All experiments were performed in triplicates. Results are presented as the mean $\pm \mathrm{SD}$. $* \mathrm{p}<0.05$ as determined by an unpaired Student's t-test, $\mathrm{n}=6$. 


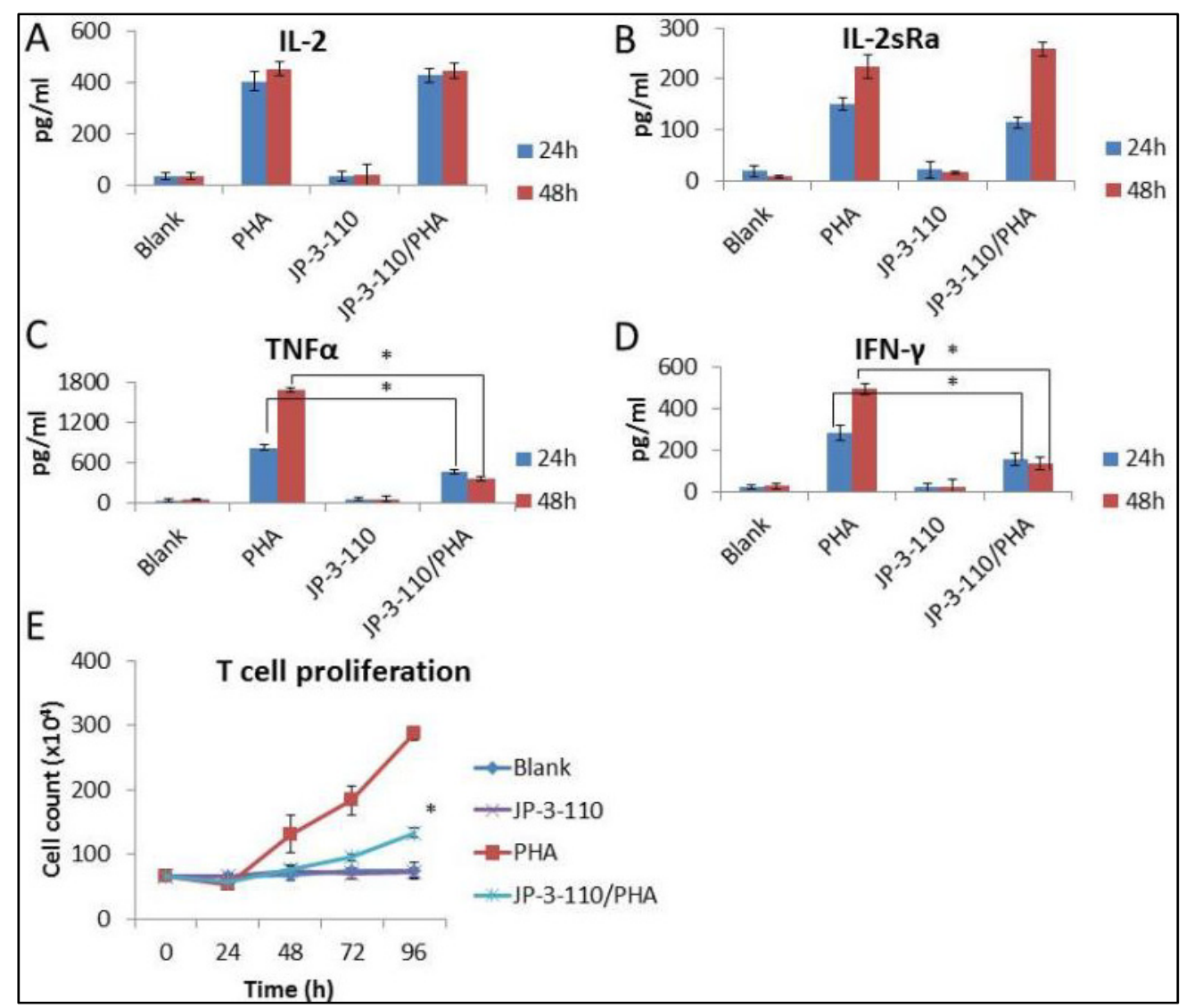

Figure 7-7. JP-3-110 suppressed $T$ cell proliferation but not $T$ cell activation A-D. The levels of IL-2, IL-2 receptor, TNF $\alpha$ and IFN $\gamma$ of T cells following PHA stimulation and the treatment JP-3-110 $(5 \mu \mathrm{M})$. The levels of IL-2 and IL-2 receptor were not affected by the treatment of JP-3-110 but the levels of TNF $\alpha$ and IFN $\gamma$ were significantly reduced. $\mathrm{E}$. The T cell proliferation after PHA stimulation is characterized by a quick drop in the early stage $(24 \mathrm{~h})$ and a following fast-proliferation stage. The presence of JP-3-110 significantly reduced the T cell proliferation after PHA stimulation. 
following activation therefore the results of less expressions of TNF $\alpha$ and IFN $\gamma$ might be due to less number of total T cells (Figure 7-7) which is consistence with previous reports $[159,160]$. Taking together, the results indicated that JP-3-110 retained the immunosuppressive function similar to MPA.

\section{JP-3-110 inhibited the function of $T$ cells and $B$ cells in humanized NSG mice}

NSG mice were adoptively transferred human PBMCs to build human immunity as described in Chapter 6. The human IgG level in the serum and CD3+ T cell staining in the spleen indicated that NSG mice can be readily humanized by injecting $5 \times 10^{6}$ human PBMCs (Figure 7-8). No sign of GvHD was observed after humanization. To determine whether JP-3-110 can suppress immune reactivity in humanized NSG mice, we administered JP-3-110 intraperitoneally at $50 \mathrm{mg} / \mathrm{kg}$ for consecutive 7 shots. Results indicated that the amount of human T cells in the spleen of humanized NSG mice were significantly reduced after JP-3-110 administration (Figure 7-8B). Serum human IgG level of the mice receiving JP-3-110 was also significantly reduced compared with the mice receiving equal amount of saline (Figure 7-8C), suggesting that JP-3-110 suppressed the function of T cells and B cells in humanized NSG mice.

\section{Discussion}

The idea of synthesizing a compound drug to prevent immune rejection and PNF of human islet transplantation simultaneously emerged from the research in BMSC mediated cell therapy. BMSC effective prevented the immune rejection and PNF as an immune modulator and trophic mediator, respectively. However, BMSC was unsuitable for intravenous injection therefore can only be co-transplanted with human islets.

All these drawbacks of BMSCs encouraged us to find an alternative for BMSC based stem cell therapy. In our most recent work, we proposed to take advantage of immunosuppressive drug and anti-apoptotic drug to test whether we can synthesize a compound drug to prevent immune rejection and PNF of human islet transplantation simultaneously.

Most immunosuppressive drugs have significant side effects. Therefore the dosing of these compounds in the patients is closely monitored and controlled. Among these immunosuppressive compounds, MPA is a commonly used for organ transplantation especially human islet transplantation. MPA inhibits the purine synthesis and thereby the proliferation of T cells and B cells [161]. However, MPA also impaired the viability and function of human islets in the coculture condition [87]. Hereby we reported a new compound JP-3-110, which combines the immunosuppressive drug MPA with a novel anti-apoptotic compound KZ41. We hypothesized that on one hand, the compound drug can used as a modified MPA so that the toxicity of MPA can be significantly reduced. One the other hand, the conjugated part, KZ41 had antiapoptotic potential to prevent the PNF of human islet transplantation. 


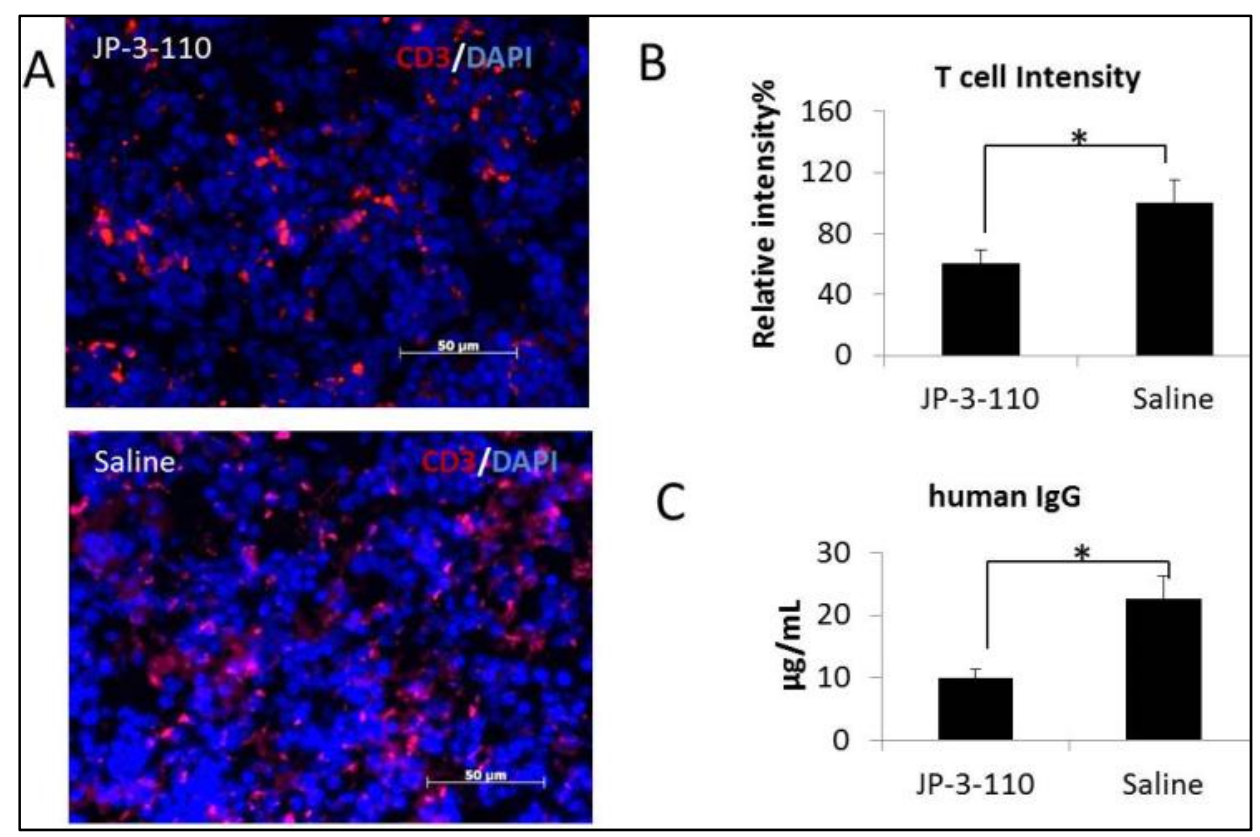

Figure 7-8. JP-3-110 inhibited the function of $\mathbf{T}$ cells and B cells in humanized NSG mice

A. A representative spleen section of humanized NSG mice after receiving 7 consecutive shots of JP-3-110 (50mg/kg, upper) and equal amount of saline (lower) each day. NSG mice were humanized by intraperitoneal injection of $5 \times 10^{6}$ human PBMCs and allowed to settle for two weeks. Sections were stained for CD3 positive T cells (red) and black stained with DAPI (blue). B. The T cell intensity in the spleen section of humanized NSG mice calculated from the immunofluorescence staining by ImageJ. C. The serum IgG level of humanized NSG mice. $\mathrm{P}<0.05$ under student $\mathrm{t}$ test. $\mathrm{N}=3$. 
We demonstrated that the compound drug JP-3-110 had immunosuppressive effect as its counterpart MPA in the MLR. Although JP-3-110 did not prevent the activation of $\mathrm{T}$ cells by PHA, it did prevent the proliferation of $\mathrm{T}$ cells after activation (Figure 7-7). To better fit the scope of clinical human islet transplantation, we employed a novel humanized mouse model to evaluate the effectiveness of the JP-3-110 to prevent the immune rejection caused by human immunity. The staining of CD3+ human cells in the spleen section and the level of human IgG in the serum of NSG mice both suggested that JP-3-110 suppressed the alloreactivity of T cells and B cells in vivo (Figure 7-8). This study may provide useful insight into the modification of traditional immunosuppressive drugs.

Previous studies indicated that KZ41 is a potent suppressor of NF-kB activity of human alveolar A579 cells and thus a potent anti-inflammatory compound [157]. We demonstrated that the anti-inflammatory effect of KZ41 can be transferred into insulinproducing INS-1E cells and human islets against the stimulation of a cytokine cocktail (Figures 7-5, 7-6). We also demonstrated that the conjugation of KZ41 with MPA did not affect the pharmacological activities of MPA as a single drug.

Unfortunately, this work is not close to complete at this stage as we faced lots of challenges in the research of this small molecular compound. The major challenge is about the rationale of the project, how we are going to evaluate the compound drug JP-3110. As shown in Table 7-1, KZ41 inhibits the production of inflammatory cytokines through NF-kB inhibition while MPA inhibits the proliferation of T cells and B cells, suggesting a potential synergistic effect to prevent the immune rejection of human islets. However, KZ41 and MPA demonstrate opposite effects on the apoptosis of human islets. Without compelling evidences that the anti-apoptotic effect of KZ41 is significantly higher than the pro-apoptotic effect of MPA, the compound drug may not show a benefit to prevent the apoptosis of human islets. Further studies are needed to compare the antiapoptotic effect of KZ41 alone, KZ41 in the presence of MPA and JP-3-110. If KZ41 is not capable to mask the pro-apoptotic effect of MPA, JP-3-110 is just a less toxic immunosuppressant instead of a compound drug with two therapeutic potentials.

Moreover, there are other challenges regarding this project. The first issue is the solubility of JP-3-110. KZ41 is still fairly water-soluble $(\sim 0.8 \mathrm{~g} / \mathrm{mL})$, but JP-3-110 is not even close to that range with water solubility of only $0.5 \mathrm{mg} / \mathrm{mL}$. The difficulties of working with a compound of such solubility are 1) the poor solubility of JP-3-110 is unsuitable for injection into animals because a huge amount of water is needed to fully dissolve the compound and 2) a micellar or liposomal delivery system cannot be used to formulate JP-3-110 because the solubility of JP-3-110 is not that poor and JP-3-110 may leak out from the delivery system. To solve issue, we need to modify the molecular structure of JP-3-110 to drastically change the solubility toward either direction while keeping the pharmacological activities of JP-3-110.

The second issue is the stability of the compound. The ester bond of JP-3-110 is susceptible to hydrolytic degradation, releasing KZ41 and MPA as two single drugs. More than $40 \%$ of JP-3-110 in the aqueous solution will be degraded when stored in $4^{\circ} \mathrm{C}$ 
Table 7-1. The pharmacological activities of KZ41 and MPA

\begin{tabular}{lll}
\hline Pharmacological activity & KZ41 & MPA \\
\hline Apoptosis impact & $\begin{array}{l}\text { Prevents apoptosis of } \\
\text { human islets at } 5 \mu \mathrm{m}\end{array}$ & $\begin{array}{l}\text { Toxic to human islets at } \\
10 \mu \mathrm{m}\end{array}$ \\
Immune impact & $\begin{array}{l}\text { Inhibits the production of } \\
\text { inflammatory cytokines }\end{array}$ & $\begin{array}{l}\text { Prevents the proliferation } \\
\text { of T cells and B cells }\end{array}$ \\
\hline
\end{tabular}


for 1 week (Figure 7-9). Although this degradation has not caused us any problem in efficacy, it may pose potential problem in the formulation of this drug.

The third issue is the difficulty in the synthesis process therefore limited amount of JP-3-110 had been synthesized for each batch. We have synthesized a total amount of $80 \mathrm{mg}$ of JP-3-110, which was just enough for the in vitro characterization but far from enough for human islet transplantation, which last for months.

In summary, we demonstrated a proof of concept that a compound drug composing of a immunosuppressive part and an antiapoptotic part can be an effective strategy to prevent the immune rejection and PNF of human islet simultaneously. However, further effect is still needed to improve the solubility and stability of this compound and formulate it for in vivo test. 


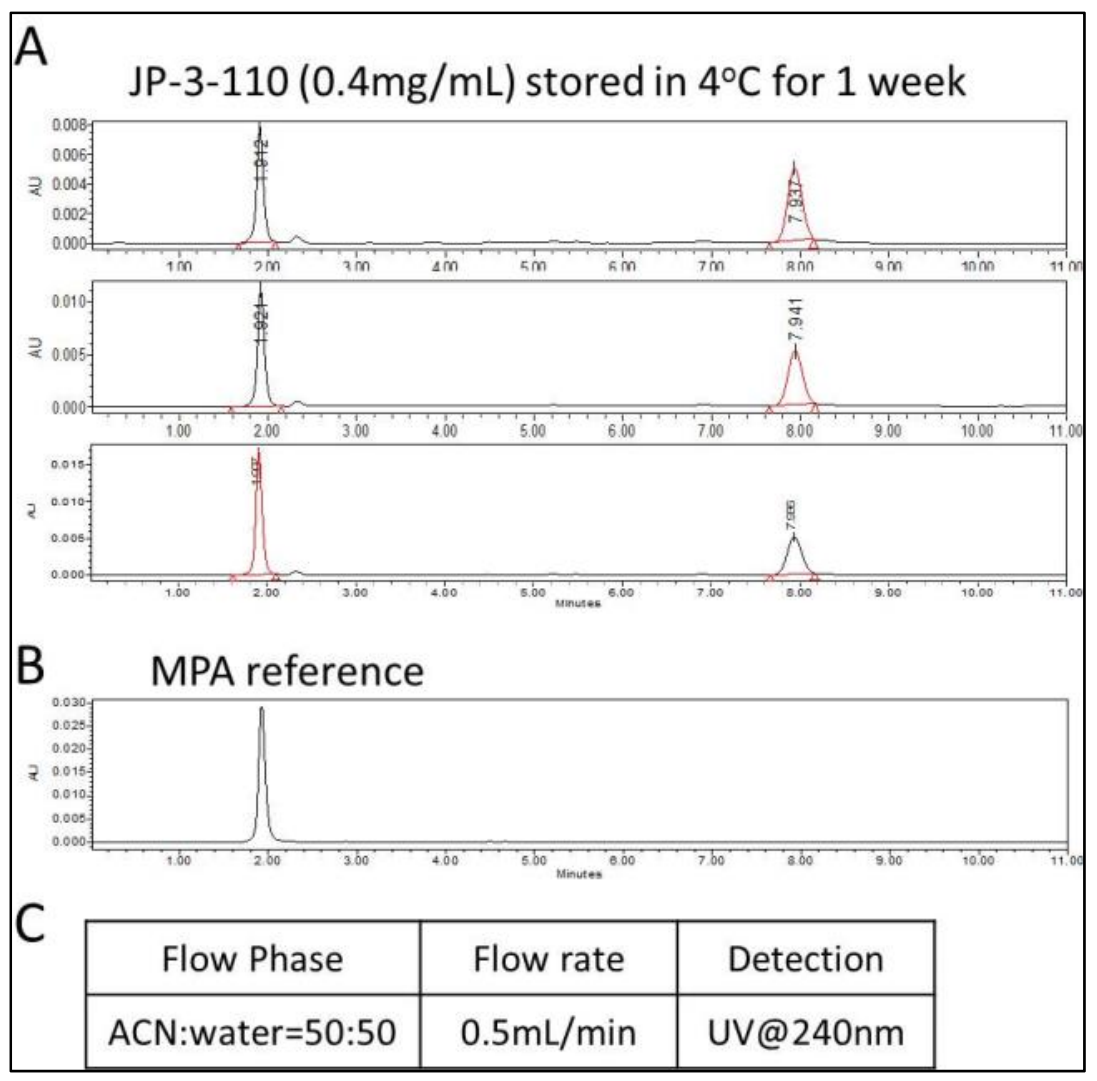

Figure 7-9. The hydrolysis of JP-3-110 in aqueous solution

A. The characterization of JP-3-110 aqueous solution $(0.4 \mathrm{mg} / \mathrm{mL})$ stored in $4^{\circ} \mathrm{C}$ for 1 week. The peak at 2 minutes was denoted as MPA degraded from JP-3-110. KZ41 does not have a benzene ring therefore cannot be detected under UV detector. B. The HPLC of MPA under a same setting as a reference. C. The HPLC setting. 


\section{CHAPTER 8. CONCLUSION}

\section{What We Achieved in the Past 5 Years}

The limited supply of human islet, the inadequate means to prevent graft rejection and the PNF remains three important obstacles for successful human islet transplantation. In the past five years, we have developed several strategies for the PNF and immune rejection. For example, we identified XIAP as a potent anti-apoptotic gene to prevent the PNF caused by inflammatory cytokines. We generated RGD modified adenovirus to achieve efficient gene transfer while reducing the viral titer. We also identified BMSC as a novel gene carrier to express therapeutic genes while avoiding the viral transduction on human islets. The establishment of humanized NSG mice has been a significant milestone in five years' research. This novel animal model provided us a clinical relevant platform to evaluate the immune rejection of human islets. Using humanized NSG mice, we successful evaluated the efficacies of BMSCs and a novel compound JP-3-110 to prevent the immune rejection of humanized mice.

In the past five years, our knowledge and skills about human islet transplantation has also been reshaped. For the strategies, we moved from gene therapy to stem cell therapy and stem cell based gene therapy. For the materials, we stopped using INS-1E cells but focused on the human islets. For the target genes, we are now targeting at least two genes simultaneously instead of one gene at a time. For the molecular cloning, we moved from buying commercially available vectors to constructing vectors and even surface-modified vectors. For in vitro islet studies, we designed and developed a new set of perifusion equipment for dynamic measurement of islet function. For the animal model, we started to use a more clinically-relevant humanized model to substitute the immunodeficient models we used before. For the transplantation techniques, we are generating a more reliable diabetic animal model and successful render a majority of diabetic mice insulin-independent after surgery.

\section{New Challenges and Future Directions}

Humanized animal model will be among the most popular animal models for the research in solid organ transplantation and immunology in the next decade. There are so many studies requiring the involvement of human immune system. Humanized model could be a perfect model for the proof of concept for these studies without the involvement of human subjects. However, the occurrence of GvHD remains a big challenge for our humanized mouse model.

The GvHD is characterized by the recognition and destruction of the major organ of mouse by the infused mature human lymphocytes. Dr. Leonard Shultz, the pioneer of humanized mouse, has reviewed his previous work and concluded that the humanized mice generated from mature human lymphocytes will develop GvHD typically in 5-7 weeks $[79,162]$. The GvHD causes short life-span and a limited window to evaluate the 
immune rejection. The only way to avoid GvHD seems is to introduce the progenitor cells of human immunocytes, which are hematopoietic stem cells into NSG mice at early age [163]. As long as the maturation of human lymphocytes happens inside the thymus or bone marrow immunodeficient mice, those mature human lymphocytes seem to be less likely to attack the mouse organs.

Gene therapy, even in its best scenario, cannot prevent the immune rejection from the islet recipient. Stem cell therapy through immunomodulatory BMSCs will be a better strategy to prevent the immune rejection. However, three weakness of stem cell therapy cannot be overlooked. First, BMSCs are unsuitable for intravenous injection, making repeated dosing impossible. Secondly, BMSC produces minimal levels of HGF and VEGF, which are not enough to support a fast and functional revascularization of human islets. Thirdly, if BMSCs from a third party were used in large quantities, they may cause immunogenicity and tumorigenicity.

To address these concerns, we raise three questions here: 1) can we expand Treg in vitro as a substitute for BMSC therapy, 2) can we modify stem cells in such a way that the therapeutic potential of stem cells can be increased and these cells can be selective cleared from body at a indicated time point and 3) can we substitute stem cells with a non-proliferation system which holds similar therapeutic potential. We believe that finding proper answers for these questions will expand our research in width and depth to further improve human islet transplantation.

The size of BMSC is quite large, making them susceptible to be sequestered in the lung after intravenous injection. Tregs are relatively smaller cells which travel freely through the bloodstream. Our data suggested that BMSCs promoted the proliferation of Tregs which in turn may contribute to the immune tolerance in human islet transplantation (Figure 6-7). Therefore, if we isolate and expand Tregs in vitro and infused back into the diabetic patient, we can increase the immune tolerance of human islets to a similar extent. Two groups in University of Chicago and University of Maryland have independently reported their preliminary data in rodent model to support this hypothesis $[164,165]$.

We demonstrated that BMSCs supported the revascularization of human islets in immune deficient NOD-SCID mice (Figure 5-9). However, our results suggested that BMSCs produce marginal level of HGF and VEGF which are incapable to support a fast and functional revascularization. Therefore, one possible outcome might be that the islets which are not destructed by the immune rejection will be PNF due to poor revascularization. In fact, we are thinking about using genetically modified BMSCs to prevent the immune rejection and PNF in humanized mouse model. To proceed with this idea, we must prove that genetic modifications, either with adenoviruses or plasmids, do not change the stem-cell-like properties and the immunomodulatory effect of BMSCs. Since telomerase reverse transcriptase (TERT)-modified BMSCs have been reported to induce tumor in animal models [55], the tumorigenicity of genetically modified BMSCs should be evaluated as well. 
For the last question whether a non-proliferative system with similar therapeutic effect can be used to substitute BMSC, we believe that the exosome secreted from BMSC can be good candidate. Exosomes are 40 to $100 \mathrm{~nm}$ vesicles secreted by a wide range of mammalian cell types, including BMSC. Exosomes also have a way to avoid the reticulo-endothelial system (RES) system [166]. These features make exosome good candidate for intravenous delivery. Exosomes typically contain proteins and RNA and therefore can be used for a wide range of purpose. For example, exosomes be used as a bridge connecting BMSC mediated stem cell therapy and adenovirus mediated gene therapy as shown in Figure 8-1.

In this ideal application of exosomes for human islet transplantation, step 2 can be further improved if we can insert genes of interest and target-ligand expression cassette into one single adenoviral vector. Step 5 needs to be optimized experimentally as the necessary dose for an optimal transplantation outcome should be determined. Since our preliminary data suggested that the BMSCs suppressed the proliferation and activation of PBMCs through soluble factors (Figure 6-3). We expect that the exosomes secreted by BMSC had similar immunomodulatory effect. Moreover, since we can introduce any gene of interest which may increase the resistance of islets to PNF, we expected that the exosome therapy would provide additional benefit compared with BMSCs therapy alone.

Exosomes mainly delivery secreted proteins and RNA. In our preliminary study we demonstrated that the adenoviral transduction significantly increased the total expression of HGF by BMSCs. Among the total HGF production, a majority stayed in the exosomes instead of being directly released into the media. These results suggested two important facts that 1) the expression of HGF by BMSCs is mainly through exosomes and 2) exosomes from genetically modified BMSCs hold therapeutic advantage above exosomes from primary BMSCs.

Future studies regarding exosomes generated from BMSCs can be approached from two directions. First, we will identify the immunosuppressive factor(s) produced by BMSCs and generate the exosomes to deliver the factor(s). If we failed to identify any of the immunosuppressive factors, we can hijack the cellular machinery of BMSCs with adenoviral vectors to produce exosomes containing well-known immunosuppressive factors such as IL-10 or TGF $\beta$. The second direction is to modify the exosomes with targeting ligands for targeted delivery to the transplantation site. The glucagon-like peptide 1 receptor (GLP1R) is one well-known receptor of pancreatic $\beta$ cells. Itoh et al. recently reported high expression of a type of G protein-coupled receptor GPR40 on the surface of pancreatic $\beta$ cells [167]. These receptors can be potential candidate for the targeted delivery of exosomes to human islets in vivo. 


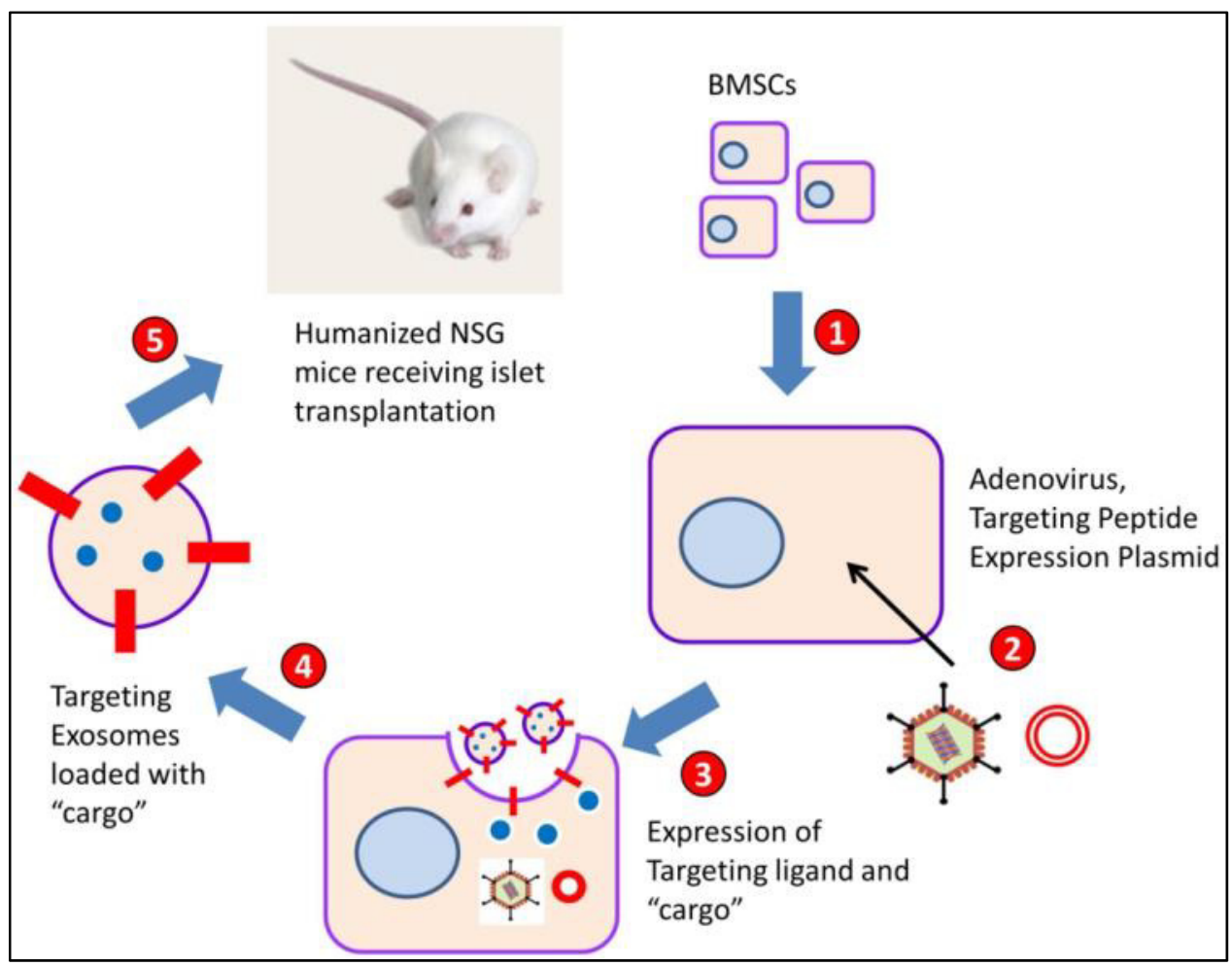

Figure 8-1. An ideal application of targeting exosomes for islet transplantation in humanized NSG mice

Step 1: Isolation and expansion of primary BMSCs. Step 2: Gene transfer into BMSCs. Adenoviral vectors encoding genes of interest and a plasmid encoding targeting ligand were transferred together into BMSCs. Step 3: Expression of targeting ligand and "cargo". Targeting ligands (red) are typically expression on the membrane and released with exosomes. "Cargo" (blue) can be secreted proteins if an expression adenoviral vector is used or shRNA if a silencing adenoviral vector is used. Step 4: Harvest of the targeting exosomes. Step 5: Intravenous injection of exosomes into humanized NSG mice receiving islet transplantation. 


\section{LIST OF REFERENCES}

1. Shapiro, AM, Lakey, JR, Ryan, EA, Korbutt, GS, Toth, E, Warnock, GL, et al. (2000). Islet transplantation in seven patients with type 1 diabetes mellitus using a glucocorticoid-free immunosuppressive regimen. The New England journal of medicine 343: 230-238.

2. Naftanel, MA, and Harlan, DM (2004). Pancreatic islet transplantation. PLoS medicine 1: e58; quiz e75.

3. Giannoukakis, N, Rudert, WA, Ghivizzani, SC, Gambotto, A, Ricordi, C, Trucco, M, et al. (1999). Adenoviral gene transfer of the interleukin-1 receptor antagonist protein to human islets prevents IL-1beta-induced beta-cell impairment and activation of islet cell apoptosis in vitro. Diabetes 48: 1730-1736.

4. Panakanti, R, and Mahato, RI (2009). Bipartite vector encoding hVEGF and hIL$1 \mathrm{Ra}$ for ex vivo transduction into human islets. Molecular pharmaceutics 6: 274284.

5. Li, F, and Mahato, RI (2008). iNOS gene silencing prevents inflammatory cytokine-induced beta-cell apoptosis. Molecular pharmaceutics 5: 407-417.

6. Cheng, G, Zhu, L, and Mahato, RI (2008). Caspase-3 gene silencing for inhibiting apoptosis in insulinoma cells and human islets. Molecular pharmaceutics 5: 10931102.

7. Narang, AS, Sabek, O, Gaber, AO, and Mahato, RI (2006). Co-expression of vascular endothelial growth factor and interleukin-1 receptor antagonist improves human islet survival and function. Pharmaceutical research 23: 1970-1982.

8. Panakanti, R, and Mahato, RI (2009). Bipartite adenoviral vector encoding hHGF and hIL-1Ra for improved human islet transplantation. Pharmaceutical research 26: 587-596.

9. Garcia-Ocana, A, Takane, KK, Reddy, VT, Lopez-Talavera, JC, Vasavada, RC, and Stewart, AF (2003). Adenovirus-mediated hepatocyte growth factor expression in mouse islets improves pancreatic islet transplant performance and reduces beta cell death. J Biol Chem 278: 343-351.

10. Ozawa, CR, Banfi, A, Glazer, NL, Thurston, G, Springer, ML, Kraft, PE, et al. (2004). Microenvironmental VEGF concentration, not total dose, determines a threshold between normal and aberrant angiogenesis. J Clin Invest 113: 516-527. 
11. Garcia-Ocana, A, Vasavada, RC, Cebrian, A, Reddy, V, Takane, KK, LopezTalavera, JC, et al. (2001). Transgenic overexpression of hepatocyte growth factor in the beta-cell markedly improves islet function and islet transplant outcomes in mice. Diabetes 50: 2752-2762.

12. Narang, AS, Cheng, K, Henry, J, Zhang, C, Sabek, O, Fraga, D, et al. (2004). Vascular endothelial growth factor gene delivery for revascularization in transplanted human islets. Pharmaceutical research 21: 15-25.

13. Mahato, RI, Henry, J, Narang, AS, Sabek, O, Fraga, D, Kotb, M, et al. (2003). Cationic lipid and polymer-based gene delivery to human pancreatic islets. Molecular therapy : the journal of the American Society of Gene Therapy 7: 89100.

14. Wu, H, Yoon, AR, Li, F, Yun, CO, and Mahato, RI (2011). RGD peptidemodified adenovirus expressing hepatocyte growth factor and X-linked inhibitor of apoptosis improves islet transplantation. The journal of gene medicine 13: 658669.

15. Wu, H, Panakanti, R, Li, F, and Mahato, RI (2010). XIAP gene expression protects beta-cells and human islets from apoptotic cell death. Molecular pharmaceutics 7: 1655-1666.

16. Emamaullee, JA, Rajotte, RV, Liston, P, Korneluk, RG, Lakey, JR, Shapiro, AM, et al. (2005). XIAP overexpression in human islets prevents early posttransplant apoptosis and reduces the islet mass needed to treat diabetes. Diabetes 54: 25412548.

17. Narang, AS, and Mahato, RI (2006). Biological and biomaterial approaches for improved islet transplantation. Pharmacological reviews 58: 194-243.

18. Sharma, S, Jhala, US, Johnson, T, Ferreri, K, Leonard, J, and Montminy, M (1997). Hormonal regulation of an islet-specific enhancer in the pancreatic homeobox gene STF-1. Molecular and cellular biology 17: 2598-2604.

19. Li, F, and Mahato, RI (2009). Bipartite vectors for co-expression of a growth factor cDNA and short hairpin RNA against an apoptotic gene. The journal of gene medicine 11: 764-771.

20. Armentano, D, Sookdeo, CC, Hehir, KM, Gregory, RJ, St George, JA, Prince, GA, et al. (1995). Characterization of an adenovirus gene transfer vector containing an E4 deletion. Human gene therapy 6: 1343-1353. 
21. Chen, HH, Mack, LM, Kelly, R, Ontell, M, Kochanek, S, and Clemens, PR (1997). Persistence in muscle of an adenoviral vector that lacks all viral genes. Proceedings of the National Academy of Sciences of the United States of America 94: $1645-1650$.

22. Zuk, PA, Zhu, M, Mizuno, H, Huang, J, Futrell, JW, Katz, AJ, et al. (2001). Multilineage cells from human adipose tissue: implications for cell-based therapies. Tissue Eng 7: 211-228.

23. Noth, U, Osyczka, AM, Tuli, R, Hickok, NJ, Danielson, KG, and Tuan, RS (2002). Multilineage mesenchymal differentiation potential of human trabecular bone-derived cells. J Orthop Res 20: 1060-1069.

24. De Bari, C, Dell'Accio, F, Tylzanowski, P, and Luyten, FP (2001). Multipotent mesenchymal stem cells from adult human synovial membrane. Arthritis Rheum 44: 1928-1942.

25. Bosch, P, Musgrave, DS, Lee, JY, Cummins, J, Shuler, T, Ghivizzani, TC, et al. (2000). Osteoprogenitor cells within skeletal muscle. J Orthop Res 18: 933-944.

26. Miura, M, Gronthos, S, Zhao, M, Lu, B, Fisher, LW, Robey, PG, et al. (2003). SHED: stem cells from human exfoliated deciduous teeth. Proceedings of the National Academy of Sciences of the United States of America 100: 5807-5812.

27. Lee, OK, Kuo, TK, Chen, WM, Lee, KD, Hsieh, SL, and Chen, TH (2004). Isolation of multipotent mesenchymal stem cells from umbilical cord blood. Blood 103: 1669-1675.

28. Musina, RA, Bekchanova, ES, Belyavskii, AV, and Sukhikh, GT (2006). Differentiation potential of mesenchymal stem cells of different origin. Bull Exp Biol Med 141: 147-151.

29. Tuan, RS, Boland, G, and Tuli, R (2003). Adult mesenchymal stem cells and cellbased tissue engineering. Arthritis Res Ther 5: 32-45.

30. Nussbaum, J, Minami, E, Laflamme, MA, Virag, JA, Ware, CB, Masino, A, et al. (2007). Transplantation of undifferentiated murine embryonic stem cells in the heart: teratoma formation and immune response. FASEB J 21: 1345-1357.

31. Rubio, D, Garcia-Castro, J, Martin, MC, de la Fuente, R, Cigudosa, JC, Lloyd, AC, et al. (2005). Spontaneous human adult stem cell transformation. Cancer Res 65: 3035-3039.

32. Le Blanc, K, Rasmusson, I, Sundberg, B, Gotherstrom, C, Hassan, M, Uzunel, M, et al. (2004). Treatment of severe acute graft-versus-host disease with third party haploidentical mesenchymal stem cells. Lancet 363: 1439-1441. 
33. Horwitz, EM, Prockop, DJ, Fitzpatrick, LA, Koo, WW, Gordon, PL, Neel, M, et al. (1999). Transplantability and therapeutic effects of bone marrow-derived mesenchymal cells in children with osteogenesis imperfecta. Nat Med 5: 309-313.

34. Wakitani, S, Nawata, M, Tensho, K, Okabe, T, Machida, H, and Ohgushi, H (2007). Repair of articular cartilage defects in the patello-femoral joint with autologous bone marrow mesenchymal cell transplantation: three case reports involving nine defects in five knees. J Tissue Eng Regen Med 1: 74-79.

35. Young, RG, Butler, DL, Weber, W, Caplan, AI, Gordon, SL, and Fink, DJ (1998). Use of mesenchymal stem cells in a collagen matrix for Achilles tendon repair. J Orthop Res 16: 406-413.

36. Prockop, DJ (1997). Marrow stromal cells as stem cells for nonhematopoietic tissues. Science 276: 71-74.

37. Mangi, AA, Noiseux, N, Kong, D, He, H, Rezvani, M, Ingwall, JS, et al. (2003). Mesenchymal stem cells modified with Akt prevent remodeling and restore performance of infarcted hearts. Nat Med 9: 1195-1201.

38. De Bari, C, Dell'Accio, F, Vandenabeele, F, Vermeesch, JR, Raymackers, JM, and Luyten, FP (2003). Skeletal muscle repair by adult human mesenchymal stem cells from synovial membrane. J Cell Biol 160: 909-918.

39. Koc, ON, Gerson, SL, Cooper, BW, Dyhouse, SM, Haynesworth, SE, Caplan, AI, et al. (2000). Rapid hematopoietic recovery after coinfusion of autologous-blood stem cells and culture-expanded marrow mesenchymal stem cells in advanced breast cancer patients receiving high-dose chemotherapy. J Clin Oncol 18: 307316.

40. Pittenger, MF, Mackay, AM, Beck, SC, Jaiswal, RK, Douglas, R, Mosca, JD, et al. (1999). Multilineage potential of adult human mesenchymal stem cells. Science 284: 143-147.

41. Ezquer, FE, Ezquer, ME, Parrau, DB, Carpio, D, Yanez, AJ, and Conget, PA (2008). Systemic administration of multipotent mesenchymal stromal cells reverts hyperglycemia and prevents nephropathy in type 1 diabetic mice. Biol Blood Marrow Transplant 14: 631-640.

42. Lee, RH, Seo, MJ, Reger, RL, Spees, JL, Pulin, AA, Olson, SD, et al. (2006). Multipotent stromal cells from human marrow home to and promote repair of pancreatic islets and renal glomeruli in diabetic NOD/scid mice. Proceedings of the National Academy of Sciences of the United States of America 103: 1743817443. 
43. Urban, VS, Kiss, J, Kovacs, J, Gocza, E, Vas, V, Monostori, E, et al. (2008). Mesenchymal stem cells cooperate with bone marrow cells in therapy of diabetes. Stem Cells 26: 244-253.

44. Hess, D, Li, L, Martin, M, Sakano, S, Hill, D, Strutt, B, et al. (2003). Bone marrow-derived stem cells initiate pancreatic regeneration. Nat Biotechnol 21: 763-770.

45. Choi, JB, Uchino, H, Azuma, K, Iwashita, N, Tanaka, Y, Mochizuki, H, et al. (2003). Little evidence of transdifferentiation of bone marrow-derived cells into pancreatic beta cells. Diabetologia 46: 1366-1374.

46. Dor, Y, Brown, J, Martinez, OI, and Melton, DA (2004). Adult pancreatic betacells are formed by self-duplication rather than stem-cell differentiation. Nature 429: 41-46.

47. Hayek, A, Beattie, GM, Cirulli, V, Lopez, AD, Ricordi, C, and Rubin, JS (1995). Growth factor/matrix-induced proliferation of human adult beta-cells. Diabetes 44: $1458-1460$.

48. Tang, YL, Zhao, Q, Zhang, YC, Cheng, L, Liu, M, Shi, J, et al. (2004). Autologous mesenchymal stem cell transplantation induce VEGF and neovascularization in ischemic myocardium. Regul Pept 117: 3-10.

49. Izumida, Y, Aoki, T, Yasuda, D, Koizumi, T, Suganuma, C, Saito, K, et al. (2005). Hepatocyte growth factor is constitutively produced by donor-derived bone marrow cells and promotes regeneration of pancreatic beta-cells. Biochem Biophys Res Commun 333: 273-282.

50. Sordi, V, Melzi, R, Mercalli, A, Formicola, R, Doglioni, C, Tiboni, F, et al. (2010). Mesenchymal cells appearing in pancreatic tissue culture are bone marrow-derived stem cells with the capacity to improve transplanted islet function. Stem Cells 28: 140-151.

51. Rackham, CL, Chagastelles, PC, Nardi, NB, Hauge-Evans, AC, Jones, PM, and King, AJ (2011). Co-transplantation of mesenchymal stem cells maintains islet organisation and morphology in mice. Diabetologia.

52. Wu, H, Lu, W, and Mahato, RI (2011). Mesenchymal stem cells as a gene delivery vehicle for successful islet transplantation. Pharmaceutical research 28: 2098-2109.

53. Silva, GV, Litovsky, S, Assad, JA, Sousa, AL, Martin, BJ, Vela, D, et al. (2005). Mesenchymal stem cells differentiate into an endothelial phenotype, enhance vascular density, and improve heart function in a canine chronic ischemia model. Circulation 111: 150-156. 
54. Ito, T, Itakura, S, Todorov, I, Rawson, J, Asari, S, Shintaku, J, et al. (2010).

Mesenchymal stem cell and islet co-transplantation promotes graft revascularization and function. Transplantation 89: 1438-1445.

55. Ryan, JM, Barry, FP, Murphy, JM, and Mahon, BP (2005). Mesenchymal stem cells avoid allogeneic rejection. J Inflamm (Lond) 2: 8.

56. Mackay, AM, Beck, SC, Murphy, JM, Barry, FP, Chichester, CO, and Pittenger, MF (1998). Chondrogenic differentiation of cultured human mesenchymal stem cells from marrow. Tissue Eng 4: 415-428.

57. Murphy, JM, Fink, DJ, Hunziker, EB, and Barry, FP (2003). Stem cell therapy in a caprine model of osteoarthritis. Arthritis Rheum 48: 3464-3474.

58. Di Nicola, M, Carlo-Stella, C, Magni, M, Milanesi, M, Longoni, PD, Matteucci, $\mathrm{P}$, et al. (2002). Human bone marrow stromal cells suppress T-lymphocyte proliferation induced by cellular or nonspecific mitogenic stimuli. Blood 99: 3838-3843.

59. Krampera, M, Glennie, S, Dyson, J, Scott, D, Laylor, R, Simpson, E, et al. (2003). Bone marrow mesenchymal stem cells inhibit the response of naive and memory antigen-specific T cells to their cognate peptide. Blood 101: 3722-3729.

60. Ding, Y, Xu, D, Feng, G, Bushell, A, Muschel, RJ, and Wood, KJ (2009). Mesenchymal stem cells prevent the rejection of fully allogenic islet grafts by the immunosuppressive activity of matrix metalloproteinase-2 and -9 . Diabetes 58 : 1797-1806.

61. Crop, MJ, Baan, CC, Korevaar, SS, Ijzermans, JN, Alwayn, IP, Weimar, W, et al. (2009). Donor-derived mesenchymal stem cells suppress alloreactivity of kidney transplant patients. Transplantation 87: 896-906.

62. Popp, FC, Eggenhofer, E, Renner, P, Slowik, P, Lang, SA, Kaspar, H, et al. (2008). Mesenchymal stem cells can induce long-term acceptance of solid organ allografts in synergy with low-dose mycophenolate. Transpl Immunol 20: 55-60.

63. Rasmusson, I, Ringden, O, Sundberg, B, and Le Blanc, K (2005). Mesenchymal stem cells inhibit lymphocyte proliferation by mitogens and alloantigens by different mechanisms. Exp Cell Res 305: 33-41.

64. Aggarwal, S, and Pittenger, MF (2005). Human mesenchymal stem cells modulate allogeneic immune cell responses. Blood 105: 1815-1822.

65. Beyth, S, Borovsky, Z, Mevorach, D, Liebergall, M, Gazit, Z, Aslan, H, et al. (2005). Human mesenchymal stem cells alter antigen-presenting cell maturation and induce T-cell unresponsiveness. Blood 105: 2214-2219. 
66. Tse, WT, Pendleton, JD, Beyer, WM, Egalka, MC, and Guinan, EC (2003). Suppression of allogeneic T-cell proliferation by human marrow stromal cells: implications in transplantation. Transplantation 75: 389-397.

67. Longoni, B, Szilagyi, E, Quaranta, P, Paoli, GT, Tripodi, S, Urbani, S, et al. (2010). Mesenchymal stem cells prevent acute rejection and prolong graft function in pancreatic islet transplantation. Diabetes Technol Ther 12: 435-446.

68. Li, FR, Wang, XG, Deng, CY, Qi, H, Ren, LL, and Zhou, HX (2010). Immune modulation of co-transplantation mesenchymal stem cells with islet on $\mathrm{T}$ and dendritic cells. Clin Exp Immunol 161: 357-363.

69. Kim, YH, Wee, YM, Choi, MY, Lim, DG, Kim, SC, and Han, DJ (2011). IL-10 induced by $\mathrm{CD} 1 \mathrm{~b}+\mathrm{bells}$ and IL-10 activated regulatory $\mathrm{T}$ cells play a role in immune modulation of mesenchymal stem cells in rat islet allograft. Mol Med.

70. Perico, N, Casiraghi, F, Introna, M, Gotti, E, Todeschini, M, Cavinato, RA, et al. (2011). Autologous mesenchymal stromal cells and kidney transplantation: a pilot study of safety and clinical feasibility. Clin J Am Soc Nephrol 6: 412-422.

71. Bartholomew, A, Sturgeon, C, Siatskas, M, Ferrer, K, McIntosh, K, Patil, S, et al. (2002). Mesenchymal stem cells suppress lymphocyte proliferation in vitro and prolong skin graft survival in vivo. Exp Hematol 30: 42-48.

72. Zhou, HP, Yi, DH, Yu, SQ, Sun, GC, Cui, Q, Zhu, HL, et al. (2006). Administration of donor-derived mesenchymal stem cells can prolong the survival of rat cardiac allograft. Transplant Proc 38: 3046-3051.

73. Casiraghi, F, Azzollini, N, Cassis, P, Imberti, B, Morigi, M, Cugini, D, et al. (2008). Pretransplant infusion of mesenchymal stem cells prolongs the survival of a semiallogeneic heart transplant through the generation of regulatory T cells. $J$ Immunol 181: 3933-3946.

74. Solari, MG, Srinivasan, S, Boumaza, I, Unadkat, J, Harb, G, Garcia-Ocana, A, et al. (2009). Marginal mass islet transplantation with autologous mesenchymal stem cells promotes long-term islet allograft survival and sustained normoglycemia. J Autoimmun 32: 116-124.

75. Berman, DM, Willman, MA, Han, D, Kleiner, G, Kenyon, NM, Cabrera, O, et al. (2010). Mesenchymal stem cells enhance allogeneic islet engraftment in nonhuman primates. Diabetes 59: 2558-2568.

76. Chen, LB, Jiang, XB, and Yang, L (2004). Differentiation of rat marrow mesenchymal stem cells into pancreatic islet beta-cells. World J Gastroenterol 10: 3016-3020. 
77. Karnieli, O, Izhar-Prato, Y, Bulvik, S, and Efrat, S (2007). Generation of insulinproducing cells from human bone marrow mesenchymal stem cells by genetic manipulation. Stem Cells 25: 2837-2844.

78. Li, Y, Zhang, R, Qiao, H, Zhang, H, Wang, Y, Yuan, H, et al. (2007). Generation of insulin-producing cells from PDX-1 gene-modified human mesenchymal stem cells. J Cell Physiol 211: 36-44.

79. Brehm, MA, Shultz, LD, and Greiner, DL (2010). Humanized mouse models to study human diseases. Current opinion in endocrinology, diabetes, and obesity 17 : 120-125.

80. Brehm, MA, Bortell, R, Diiorio, P, Leif, J, Laning, J, Cuthbert, A, et al. (2010). Human immune system development and rejection of human islet allografts in spontaneously diabetic NOD-Rag1null IL2rgammanull Ins2Akita mice. Diabetes 59: 2265-2270.

81. Pavlakis, M, and Khwaja, K (2006). Transplantation for type 1 diabetes: whole organ pancreas and islet cells. Curr Diab Rep 6: 473-478.

82. Pawelec, K, Juszczak, MT, Kumar, A, Powis, SH, and Press, M (2008). Time course of islet loss after intraportal transplantation. Ann N Y Acad Sci 1150: 230233.

83. Takahashi, R, Deveraux, Q, Tamm, I, Welsh, K, Assa-Munt, N, Salvesen, GS, et al. (1998). A single BIR domain of XIAP sufficient for inhibiting caspases. J Biol Chem 273: 7787-7790.

84. Riedl, SJ, Renatus, M, Schwarzenbacher, R, Zhou, Q, Sun, C, Fesik, SW, et al. (2001). Structural basis for the inhibition of caspase-3 by XIAP. Cell 104: 791800.

85. Chai, J, Shiozaki, E, Srinivasula, SM, Wu, Q, Datta, P, Alnemri, ES, et al. (2001). Structural basis of caspase-7 inhibition by XIAP. Cell 104: 769-780.

86. Emamaullee, J, Liston, P, Korneluk, RG, Shapiro, AM, and Elliott, JF (2005). XIAP overexpression in islet beta-cells enhances engraftment and minimizes hypoxia-reperfusion injury. Am J Transplant 5: 1297-1305.

87. Hui, H, Khoury, N, Zhao, X, Balkir, L, D'Amico, E, Bullotta, A, et al. (2005). Adenovirus-mediated XIAP gene transfer reverses the negative effects of immunosuppressive drugs on insulin secretion and cell viability of isolated human islets. Diabetes 54: 424-433. 
88. Plesner, A, Liston, P, Tan, R, Korneluk, RG, and Verchere, CB (2005). The Xlinked inhibitor of apoptosis protein enhances survival of murine islet allografts. Diabetes 54: 2533-2540.

89. Oberhammer, F, Wilson, JW, Dive, C, Morris, ID, Hickman, JA, Wakeling, AE, et al. (1993). Apoptotic death in epithelial cells: cleavage of DNA to 300 and/or $50 \mathrm{~kb}$ fragments prior to or in the absence of internucleosomal fragmentation. Embo J 12: 3679-3684.

90. Duckett, CS, Li, F, Wang, Y, Tomaselli, KJ, Thompson, CB, and Armstrong, RC (1998). Human IAP-like protein regulates programmed cell death downstream of Bcl-xL and cytochrome c. Molecular and cellular biology 18: 608-615.

91. Deveraux, QL, and Reed, JC (1999). IAP family proteins--suppressors of apoptosis. Genes Dev 13: 239-252.

92. Olerud, J, Kanaykina, N, Vasilovska, S, King, D, Sandberg, M, Jansson, L, et al. (2009). Neural crest stem cells increase beta cell proliferation and improve islet function in co-transplanted murine pancreatic islets. Diabetologia.

93. Nikolovska-Coleska, Z, Xu, L, Hu, Z, Tomita, Y, Li, P, Roller, PP, et al. (2004). Discovery of embelin as a cell-permeable, small-molecular weight inhibitor of XIAP through structure-based computational screening of a traditional herbal medicine three-dimensional structure database. J Med Chem 47: 2430-2440.

94. Danquah, M, Li, F, Duke, CB, 3rd, Miller, DD, and Mahato, RI (2009). Micellar delivery of bicalutamide and embelin for treating prostate cancer. Pharmaceutical research 26: 2081-2092.

95. Biarnes, M, Montolio, M, Nacher, V, Raurell, M, Soler, J, and Montanya, E (2002). Beta-cell death and mass in syngeneically transplanted islets exposed to short- and long-term hyperglycemia. Diabetes 51: 66-72.

96. Ryan, EA, Paty, BW, Senior, PA, Bigam, D, Alfadhli, E, Kneteman, NM, et al. (2005). Five-year follow-up after clinical islet transplantation. Diabetes 54: 20602069.

97. Corbett, JA, Wang, JL, Misko, TP, Zhao, W, Hickey, WF, and McDaniel, ML (1993). Nitric oxide mediates IL-1 beta-induced islet dysfunction and destruction: prevention by dexamethasone. Autoimmunity 15: 145-153.

98. Burke, JF, Jr., Pirsch, JD, Ramos, EL, Salomon, DR, Stablein, DM, Van Buren, $\mathrm{DH}$, et al. (1994). Long-term efficacy and safety of cyclosporine in renaltransplant recipients. N Engl J Med 331: 358-363. 
99. Dantal, J, Hourmant, M, Cantarovich, D, Giral, M, Blancho, G, Dreno, B, et al. (1998). Effect of long-term immunosuppression in kidney-graft recipients on cancer incidence: randomised comparison of two cyclosporin regimens. Lancet 351: 623-628.

100. Newstead, CG (1998). Assessment of risk of cancer after renal transplantation. Lancet 351: 610-611.

101. Street, CN, Lakey, JR, Shapiro, AM, Imes, S, Rajotte, RV, Ryan, EA, et al. (2004). Islet graft assessment in the Edmonton Protocol: implications for predicting long-term clinical outcome. Diabetes 53: 3107-3114.

102. Nadeau, I, Garnier, A, Cote, J, Massie, B, Chavarie, C, and Kamen, A (1996). Improvement of recombinant protein production with the human adenovirus/293S expression system using fed-batch strategies. Biotechnol Bioeng 51: 613-623.

103. Svensson, EC, Marshall, DJ, Woodard, K, Lin, H, Jiang, F, Chu, L, et al. (1999). Efficient and stable transduction of cardiomyocytes after intramyocardial injection or intracoronary perfusion with recombinant adeno-associated virus vectors. Circulation 99: 201-205.

104. von Degenfeld, G, Banfi, A, Springer, ML, Wagner, RA, Jacobi, J, Ozawa, CR, et al. (2006). Microenvironmental VEGF distribution is critical for stable and functional vessel growth in ischemia. Faseb J 20: 2657-2659.

105. Vischioni, B, van der Valk, P, Span, SW, Kruyt, FA, Rodriguez, JA, and Giaccone, G (2006). Expression and localization of inhibitor of apoptosis proteins in normal human tissues. Hum Pathol 37: 78-86.

106. Emamaullee, JA, Shapiro, AM, Rajotte, RV, Korbutt, G, and Elliott, JF (2006). Neonatal porcine islets exhibit natural resistance to hypoxia-induced apoptosis. Transplantation 82: 945-952.

107. Ahn, KS, Sethi, G, and Aggarwal, BB (2007). Embelin, an inhibitor of X chromosome-linked inhibitor-of-apoptosis protein, blocks nuclear factor-kappaB (NF-kappaB) signaling pathway leading to suppression of NF-kappaB-regulated antiapoptotic and metastatic gene products. Mol Pharmacol 71: 209-219.

108. Ricordi, C, Gray, DW, Hering, BJ, Kaufman, DB, Warnock, GL, Kneteman, NM, et al. (1990). Islet isolation assessment in man and large animals. Acta diabetologica latina 27: 185-195.

109. Yun, CO, Kim, E, Koo, T, Kim, H, Lee, YS, and Kim, JH (2005). ADPoverexpressing adenovirus elicits enhanced cytopathic effect by induction of apoptosis. Cancer Gene Ther 12: 61-71. 
110. Chartier, C, Degryse, E, Gantzer, M, Dieterle, A, Pavirani, A, and Mehtali, M (1996). Efficient generation of recombinant adenovirus vectors by homologous recombination in Escherichia coli. J Virol 70: 4805-4810.

111. Wu, H, Panakanti, R, Li, F, and Mahato, RI (2010). XIAP Gene Expression Protects beta-Cells and Human Islets from Apoptotic Cell Death. Mol Pharm.

112. Xiao, GH, Jeffers, M, Bellacosa, A, Mitsuuchi, Y, Vande Woude, GF, and Testa, JR (2001). Anti-apoptotic signaling by hepatocyte growth factor/Met via the phosphatidylinositol 3-kinase/Akt and mitogen-activated protein kinase pathways. Proceedings of the National Academy of Sciences of the United States of America 98: 247-252.

113. Huang, Y, Park, YC, Rich, RL, Segal, D, Myszka, DG, and Wu, H (2001). Structural basis of caspase inhibition by XIAP: differential roles of the linker versus the BIR domain. Cell 104: 781-790.

114. Deveraux, QL, Leo, E, Stennicke, HR, Welsh, K, Salvesen, GS, and Reed, JC (1999). Cleavage of human inhibitor of apoptosis protein XIAP results in fragments with distinct specificities for caspases. EMBO J 18: 5242-5251.

115. Bratton, SB, Lewis, J, Butterworth, M, Duckett, CS, and Cohen, GM (2002). XIAP inhibition of caspase-3 preserves its association with the Apaf-1 apoptosome and prevents CD95- and Bax-induced apoptosis. Cell Death Differ 9: 881-892.

116. Figliuzzi, M, Cornolti, R, Perico, N, Rota, C, Morigi, M, Remuzzi, G, et al. (2009). Bone marrow-derived mesenchymal stem cells improve islet graft function in diabetic rats. Transplant Proc 41: 1797-1800.

117. Haynesworth, SE, Baber, MA, and Caplan, AI (1996). Cytokine expression by human marrow-derived mesenchymal progenitor cells in vitro: effects of dexamethasone and IL-1 alpha. J Cell Physiol 166: 585-592.

118. Silva, WA, Jr., Covas, DT, Panepucci, RA, Proto-Siqueira, R, Siufi, JL, Zanette, DL, et al. (2003). The profile of gene expression of human marrow mesenchymal stem cells. Stem Cells 21: 661-669.

119. Prockop, DJ (1998). Marrow stromal cells as stem cells for continual renewal of nonhematopoietic tissues and as potential vectors for gene therapy. J Cell Biochem: 284-285.

120. Bardelli, A, Longati, P, Albero, D, Goruppi, S, Schneider, C, Ponzetto, C, et al. (1996). HGF receptor associates with the anti-apoptotic protein BAG-1 and prevents cell death. EMBO J 15: 6205-6212. 
121. Friedlander, RM, Gagliardini, V, Rotello, RJ, and Yuan, J (1996). Functional role of interleukin 1 beta (IL-1 beta) in IL-1 beta-converting enzyme-mediated apoptosis. J Exp Med 184: 717-724.

122. Nakano, M, Yasunami, Y, Maki, T, Kodama, S, Ikehara, Y, Nakamura, T, et al. (2000). Hepatocyte growth factor is essential for amelioration of hyperglycemia in streptozotocin-induced diabetic mice receiving a marginal mass of intrahepatic islet grafts. Transplantation 69: 214-221.

123. Themis, M, Waddington, SN, Schmidt, M, von Kalle, C, Wang, Y, Al-Allaf, F, et al. (2005). Oncogenesis following delivery of a nonprimate lentiviral gene therapy vector to fetal and neonatal mice. Molecular therapy : the journal of the American Society of Gene Therapy 12: 763-771.

124. Beardsley, T (2000). Gene therapy setback. Sci Am 282: 36-37.

125. Stolberg, SG (2002). Trials are halted on gene therapy: child in experiment falls ill--new setback for research. NY Times (Print): A1, A25.

126. Baksh, D, Song, L, and Tuan, RS (2004). Adult mesenchymal stem cells: characterization, differentiation, and application in cell and gene therapy. J Cell Mol Med 8: 301-316.

127. Liu, N, Chen, R, Du, H, Wang, J, Zhang, Y, and Wen, J (2009). Expression of IL10 and TNF-alpha in rats with cerebral infarction after transplantation with mesenchymal stem cells. Cell Mol Immunol 6: 207-213.

128. Guo, Z, Li, H, Li, X, Yu, X, Wang, H, Tang, P, et al. (2006). In vitro characteristics and in vivo immunosuppressive activity of compact bone-derived murine mesenchymal progenitor cells. Stem Cells 24: 992-1000.

129. Djouad, F, Plence, P, Bony, C, Tropel, P, Apparailly, F, Sany, J, et al. (2003). Immunosuppressive effect of mesenchymal stem cells favors tumor growth in allogeneic animals. Blood 102: 3837-3844.

130. van der Windt, DJ, Bottino, R, Casu, A, Campanile, N, Smetanka, C, He, J, et al. (2009). Long-term controlled normoglycemia in diabetic non-human primates after transplantation with hCD46 transgenic porcine islets. Am J Transplant 9: 2716-2726.

131. Barshes, NR, Wyllie, S, and Goss, JA (2005). Inflammation-mediated dysfunction and apoptosis in pancreatic islet transplantation: implications for intrahepatic grafts. J Leukoc Biol 77: 587-597.

132. Korbling, M, and Estrov, Z (2003). Adult stem cells for tissue repair - a new therapeutic concept? The New England journal of medicine 349: 570-582. 
133. Wang, X, Hao, J, Metzger, DL, Mui, A, Ao, Z, Verchere, CB, et al. (2009). Local expression of $\mathrm{B} 7-\mathrm{H} 4$ by recombinant adenovirus transduction in mouse islets prolongs allograft survival. Transplantation 87: 482-490.

134. Chuah, MK, Brems, H, Vanslembrouck, V, Collen, D, and Vandendriessche, T (1998). Bone marrow stromal cells as targets for gene therapy of hemophilia A. Human gene therapy 9: 353-365.

135. Allay, JA, Dennis, JE, Haynesworth, SE, Majumdar, MK, Clapp, DW, Shultz, LD, et al. (1997). LacZ and interleukin-3 expression in vivo after retroviral transduction of marrow-derived human osteogenic mesenchymal progenitors. Human gene therapy 8: 1417-1427.

136. Satoh, M, Yasunami, Y, Matsuoka, N, Nakano, M, Itoh, T, Nitta, T, et al. (2007). Successful islet transplantation to two recipients from a single donor by targeting proinflammatory cytokines in mice. Transplantation 83: 1085-1092.

137. Berman, DM, Willman, MA, Han, D, Kleiner, G, Kenyon, NM, Cabrera, O, et al. (2010). Mesenchymal Stem Cells Enhance Allogeneic Islet Engraftment in Nonhuman Primates. Diabetes.

138. Manning, E, Pham, S, Li, S, Vazquez-Padron, RI, Mathew, J, Ruiz, P, et al. (2010). Interleukin-10 delivery via mesenchymal stem cells: a novel gene therapy approach to prevent lung ischemia-reperfusion injury. Human gene therapy 21 : 713-727.

139. Yan, C, Lian, X, Dai, Y, Wang, X, Qu, P, White, A, et al. (2007). Gene delivery by the hSP-B promoter to lung alveolar type II epithelial cells in LAL-knockout mice through bone marrow mesenchymal stem cells. Gene Ther 14: 1461-1470.

140. Noguchi, H, Nakai, Y, Matsumoto, S, Kawaguchi, M, Ueda, M, Okitsu, T, et al. (2005). Cell permeable peptide of JNK inhibitor prevents islet apoptosis immediately after isolation and improves islet graft function. Am J Transplant 5: 1848-1855.

141. Omori, K, Mitsuhashi, M, Todorov, I, Rawson, J, Shiang, KD, Kandeel, F, et al. (2010). Microassay for glucose-induced preproinsulin mRNA expression to assess islet functional potency for islet transplantation. Transplantation 89: 146-154.

142. Boumaza, I, Srinivasan, S, Witt, WT, Feghali-Bostwick, C, Dai, Y, Garcia-Ocana, A, et al. (2009). Autologous bone marrow-derived rat mesenchymal stem cells promote PDX-1 and insulin expression in the islets, alter T cell cytokine pattern and preserve regulatory $\mathrm{T}$ cells in the periphery and induce sustained normoglycemia. Journal of autoimmunity 32 : 33-42. 
143. Inoue, S, Popp, FC, Koehl, GE, Piso, P, Schlitt, HJ, Geissler, EK, et al. (2006). Immunomodulatory effects of mesenchymal stem cells in a rat organ transplant model. Transplantation 81: 1589-1595.

144. Yeung, TY, Seeberger, KL, Kin, T, Adesida, A, Jomha, N, Shapiro, AM, et al. (2012). Human mesenchymal stem cells protect human islets from proinflammatory cytokines. PloS one 7: e38189.

145. Harui, A, Kiertscher, SM, and Roth, MD (2011). Reconstitution of huPBL-NSG mice with donor-matched dendritic cells enables antigen-specific T-cell activation. Journal of neuroimmune pharmacology : the official journal of the Society on NeuroImmune Pharmacology 6: 148-157.

146. Mazar, J, Thomas, M, Bezrukov, L, Chanturia, A, Pekkurnaz, G, Yin, S, et al. (2009). Cytotoxicity mediated by the Fas ligand (FasL)-activated apoptotic pathway in stem cells. J Biol Chem 284: 22022-22028.

147. Dhodapkar, MV, Steinman, RM, Krasovsky, J, Munz, C, and Bhardwaj, N (2001). Antigen-specific inhibition of effector T cell function in humans after injection of immature dendritic cells. J Exp Med 193: 233-238.

148. Issa, F, Hester, J, Goto, R, Nadig, SN, Goodacre, TE, and Wood, K (2010). Ex vivo-expanded human regulatory $\mathrm{T}$ cells prevent the rejection of skin allografts in a humanized mouse model. Transplantation 90: 1321-1327.

149. Zhang, B, Liu, R, Shi, D, Liu, X, Chen, Y, Dou, X, et al. (2009). Mesenchymal stem cells induce mature dendritic cells into a novel Jagged-2-dependent regulatory dendritic cell population. Blood 113: 46-57.

150. Weng, J, He, C, Lai, P, Luo, C, Guo, R, Wu, S, et al. (2012). Mesenchymal Stromal Cells Treatment Attenuates Dry Eye in Patients With Chronic Graftversus-host Disease. Molecular therapy : the journal of the American Society of Gene Therapy 20: 2347-2354.

151. Chan, JL, Tang, KC, Patel, AP, Bonilla, LM, Pierobon, N, Ponzio, NM, et al. (2006). Antigen-presenting property of mesenchymal stem cells occurs during a narrow window at low levels of interferon-gamma. Blood 107: 4817-4824.

152. Lodie, TA, Blickarz, CE, Devarakonda, TJ, He, C, Dash, AB, Clarke, J, et al. (2002). Systematic analysis of reportedly distinct populations of multipotent bone marrow-derived stem cells reveals a lack of distinction. Tissue Eng 8: 739-751.

153. Sato, H, Kuwashima, N, Sakaida, T, Hatano, M, Dusak, JE, Fellows-Mayle, WK, et al. (2005). Epidermal growth factor receptor-transfected bone marrow stromal cells exhibit enhanced migratory response and therapeutic potential against murine brain tumors. Cancer Gene Ther 12: 757-768. 
154. Groh, ME, Maitra, B, Szekely, E, and Koc, ON (2005). Human mesenchymal stem cells require monocyte-mediated activation to suppress alloreactive $\mathrm{T}$ cells. Exp Hematol 33: 928-934.

155. Jiang, XX, Zhang, Y, Liu, B, Zhang, SX, Wu, Y, Yu, XD, et al. (2005). Human mesenchymal stem cells inhibit differentiation and function of monocyte-derived dendritic cells. Blood 105: 4120-4126.

156. Spaggiari, GM, Capobianco, A, Abdelrazik, H, Becchetti, F, Mingari, MC, and Moretta, L (2008). Mesenchymal stem cells inhibit natural killer-cell proliferation, cytotoxicity, and cytokine production: role of indoleamine 2,3dioxygenase and prostaglandin E2. Blood 111: 1327-1333.

157. Zeng, K, Thompson, KE, Yates, CR, and Miller, DD (2009). Synthesis and biological evaluation of quinic acid derivatives as anti-inflammatory agents. Bioorg Med Chem Lett 19: 5458-5460.

158. Zeng, K, Thompson, KE, Yates, CR, and Miller, DD (2012). Anti-inflammatory quinic acid derivatives for oral administration.: United States.

159. Quemeneur, L, Flacher, M, Gerland, LM, Ffrench, M, Revillard, JP, and Bonnefoy-Berard, N (2002). Mycophenolic acid inhibits IL-2-dependent T cell proliferation, but not IL-2-dependent survival and sensitization to apoptosis. $\mathrm{J}$ Immunol 169: 2747-2755.

160. He, X, Smeets, RL, Koenen, HJ, Vink, PM, Wagenaars, J, Boots, AM, et al. (2011). Mycophenolic acid-mediated suppression of human CD4+ T cells: more than mere guanine nucleotide deprivation. American journal of transplantation : official journal of the American Society of Transplantation and the American Society of Transplant Surgeons 11: 439-449.

161. Franklin, TJ, and Cook, JM (1969). The inhibition of nucleic acid synthesis by mycophenolic acid. The Biochemical journal 113: 515-524.

162. Shultz, LD, Ishikawa, F, and Greiner, DL (2007). Humanized mice in translational biomedical research. Nature reviews Immunology 7: 118-130.

163. Watanabe, S, Ohta, S, Yajima, M, Terashima, K, Ito, M, Mugishima, H, et al. (2007). Humanized NOD/SCID/IL2Rgamma(null) mice transplanted with hematopoietic stem cells under nonmyeloablative conditions show prolonged life spans and allow detailed analysis of human immunodeficiency virus type 1 pathogenesis. J Virol 81: 13259-13264. 
164. Marek, N, Krzystyniak, A, Ergenc, I, Cochet, O, Misawa, R, Wang, LJ, et al. (2011). Coating human pancreatic islets with CD4(+)CD25(high)CD127(-) regulatory $\mathrm{T}$ cells as a novel approach for the local immunoprotection. Annals of surgery 254: 512-518; discussion 518-519.

165. Shi, Q, Lees, JR, Scott, DW, Farber, DL, and Bartlett, ST (2012). Endogenous expansion of regulatory $\mathrm{T}$ cells leads to long-term islet graft survival in diabetic NOD mice. Am J Transplant 12: 1124-1132.

166. Sun, D, Zhuang, X, Xiang, X, Liu, Y, Zhang, S, Liu, C, et al. (2010). A novel nanoparticle drug delivery system: the anti-inflammatory activity of curcumin is enhanced when encapsulated in exosomes. Molecular therapy : the journal of the American Society of Gene Therapy 18: 1606-1614.

167. Itoh, Y, and Hinuma, S (2005). GPR40, a free fatty acid receptor on pancreatic beta cells, regulates insulin secretion. Hepatology research : the official journal of the Japan Society of Hepatology 33: 171-173. 


\section{VITA}

Hao Wu was born in Shanghai, China, in 1984 to Hailin Wu and Zhengying Zhao. Upon graduating with distinction from Hefei No. 1 High School in 2002, he enrolled in Tsinghua University, Beijing, China. There He pursued a Bachelor of Science degree in Biology in June 2006. He then entered the Graduate School of Tsinghua University and obtained a Master's degree in Biology under the supervision of Dr. Lijun Du in 2008. Subsequently, he joined Dr. Ram I Mahato's lab in University of Tennessee Health Science Center in pursuit of a PhD degree in Pharmaceutics. 